\title{
Expedient Total Syntheses of Pladienolide-Derived Spliceosome Modulators
}

Derek Rhoades, ${ }^{*}, \dagger, \S$ Arnold L. Rheingold,${ }^{\perp}$ Bert W. O’Malley, ${ }^{*}, \dot{\dagger}, \S$ and Jin Wang $*, \dagger, \dot{\dagger}, \S$

\author{
Department of Pharmacology and Chemical Biology \\ \#epartment of Molecular and Cellular Biology \\ ${ }^{\S}$ Center for Drug Discovery \\ Baylor College of Medicine \\ 1 Baylor Plaza, Houston, TX 77030, United States \\ ${ }^{\perp}$ Department of Chemistry and Biochemistry \\ University of California, San Diego \\ La Jolla, CA 9209, United States
}

E-mail: drhoades@bcm.edu

\section{Table of Contents}

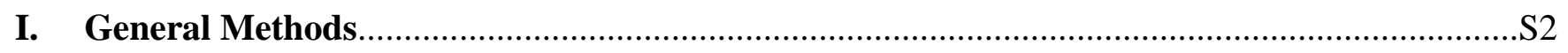

II. Summaries of Previous Total Syntheses of Pladienolides A (1) and B (2)..........................S3

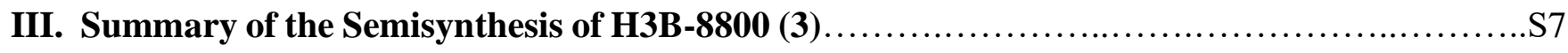

IV. Experimental Procedures and Physical Data for Compounds...........................................S8

V. Comparison of Spectroscopic Data of Natural and Synthetic Pladienolides A (1) and B (2)...S61

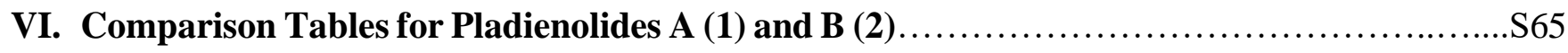

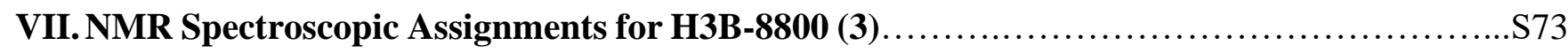

VIII. ${ }^{1} \mathrm{H},{ }^{13} \mathrm{C},{ }^{19} \mathrm{~F}$, and Selected 2D NMR Spectra of Compounds......................................S75

IX. X-Ray Crystal Structure Data for Macrolide 20 and Macrolide 24...............................S 172

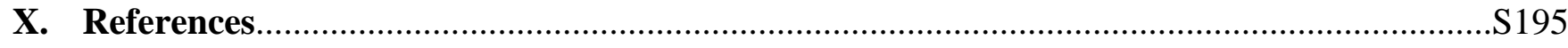




\section{Rhoades, * et al.}

\section{General Methods}

All reactions were carried out under an argon atmosphere with dry solvents under anhydrous conditions, unless otherwise noted. Anhydrous solvents (acetonitrile, dichloromethane, diethyl ether, $N, N$ dimethylformamide, hexanes, isopropanol, methanol, pyridine, tetrahydrofuran, toluene, triethylamine) were purchased from Acros Organics ${ }^{\circledR}$ (extra dry over molecular sieves, AcroSeal ${ }^{\mathrm{TM}}$ ) and used as received. Deuterated solvents were purchased from Cambridge Isotope Laboratories, Inc. Yields refer to chromatographically and spectroscopically $\left({ }^{1} \mathrm{H}\right.$ NMR) homogeneous materials, unless otherwise stated. Reagents were purchased from Sigma Aldrich or Strem Chemicals, Inc. at the highest commercial quality and used without further purification, unless otherwise stated. Reactions were monitored by thin layer chromatography (TLC) carried out on S-2 $0.25 \mathrm{~mm}$ E. Merck silica gel plates $(60 \mathrm{~F}-254,5 \times 20 \mathrm{~cm}) \mathrm{using}$ UV light $(\lambda=254 \mathrm{~nm})$ as visualizing agent and an acidic aqueous solution of $p$-anisaldehyde or a basic aqueous solution of potassium permanganate and brief heating on a hot plate as developing agents. E. Merck silica gel $60(230-400$ mesh, particle size 40-63 $\mu \mathrm{m})$ was used for flash column chromatography, ${ }^{1}$ and E. Merck silica gel plates $(60 \mathrm{~F}-254,20 \times 20 \mathrm{~cm})$ were used for preparative thin layer chromatography. NMR spectra were recorded at Rice University via the Shared Equipment Authority (SEA) on a Bruker Avance III HD $600 \mathrm{MHz}$ spectrometer equipped with a $5 \mathrm{~mm}$ DCH cryoprobe and calibrated using residual undeuterated solvent for ${ }^{1} \mathrm{H} \mathrm{NMR}\left(\mathrm{CHCl}_{3}: \delta_{\mathrm{H}}=7.26 \mathrm{ppm} ; \mathrm{CHD}_{2} \mathrm{OD}: \delta_{\mathrm{H}}=3.31\right)$, deuterated solvent for ${ }^{13} \mathrm{C} \mathrm{NMR}\left(\mathrm{CDCl}_{3}: \delta_{\mathrm{C}}=77.16 \mathrm{ppm} ; \mathrm{CD}_{3} \mathrm{OD}: \delta_{\mathrm{C}}=49.00 \mathrm{ppm}\right)$ as an internal reference at 298 $\mathrm{K},{ }^{2}$ and an external reference for ${ }^{19} \mathrm{~F}$ NMR $\left(\mathrm{CCl}_{3} \mathrm{~F}: \delta_{\mathrm{F}}=0 \mathrm{ppm}\right)$. The following abbreviations were used to designate multiplicities: $a p p=$ apparent, $b r=$ broad, $\mathrm{s}=$ singlet, $\mathrm{d}=$ doublet, $\mathrm{t}=$ triplet, $\mathrm{q}=$ quartet, $\mathrm{m}$ $=$ multiplet, $\mathrm{dd}=$ doublet of doublets, $\mathrm{ddd}=$ doublet of doublet of doublets, $\mathrm{dddd}=$ doublet of doublet of doublet of doublets, ddddd = doublet of doublet of doublet of doublet of doublets, $\mathrm{dq}=$ doublet of quartets, $\mathrm{ddq}=$ doublet of doublet of quartets, $\mathrm{dqd}=$ doublet of quartet of doublets, $\mathrm{dt}=$ doublet of triplets, $\mathrm{qdd}=$ quartet of doublets of doublets, $\mathrm{td}=$ triplet of doublets, $\mathrm{tdd}=$ triplet of doublets of doublets, $\mathrm{tt}=$ triplet of triplets. Infrared (IR) spectra were recorded on a PerkinElmer, Inc. Spectrum 100 FT-IR spectrometer. High-resolution mass spectra (HRMS) were recorded by the Mass Spectrometry Facility at the University of Texas at Austin using CI (chemical ionization), EI (electron ionization), or ESI (electrospray ionization) methods. Optical rotations were recorded on a Schmidt+Haensch POLARTRONIC M100 polarimeter at $589 \mathrm{~nm}$ and are reported in units of $10^{-1}\left(\mathrm{deg} \mathrm{cm}^{2} \mathrm{~g}^{-1}\right)$. Single crystal X-ray diffraction (XRD) analysis was performed by the Small Molecule X-ray Crystallography Facility at the University of California, San Diego. Analytical chiral reversed-phase high-performance liquid chromatography (RPHPLC) was performed using an Agilent ${ }^{\circledR} 1260$ Infinity II instrumentation apparatus. 
D. Rhoades, * et al.

\section{Summaries of Previous Total Syntheses of Pladienolides A and B}

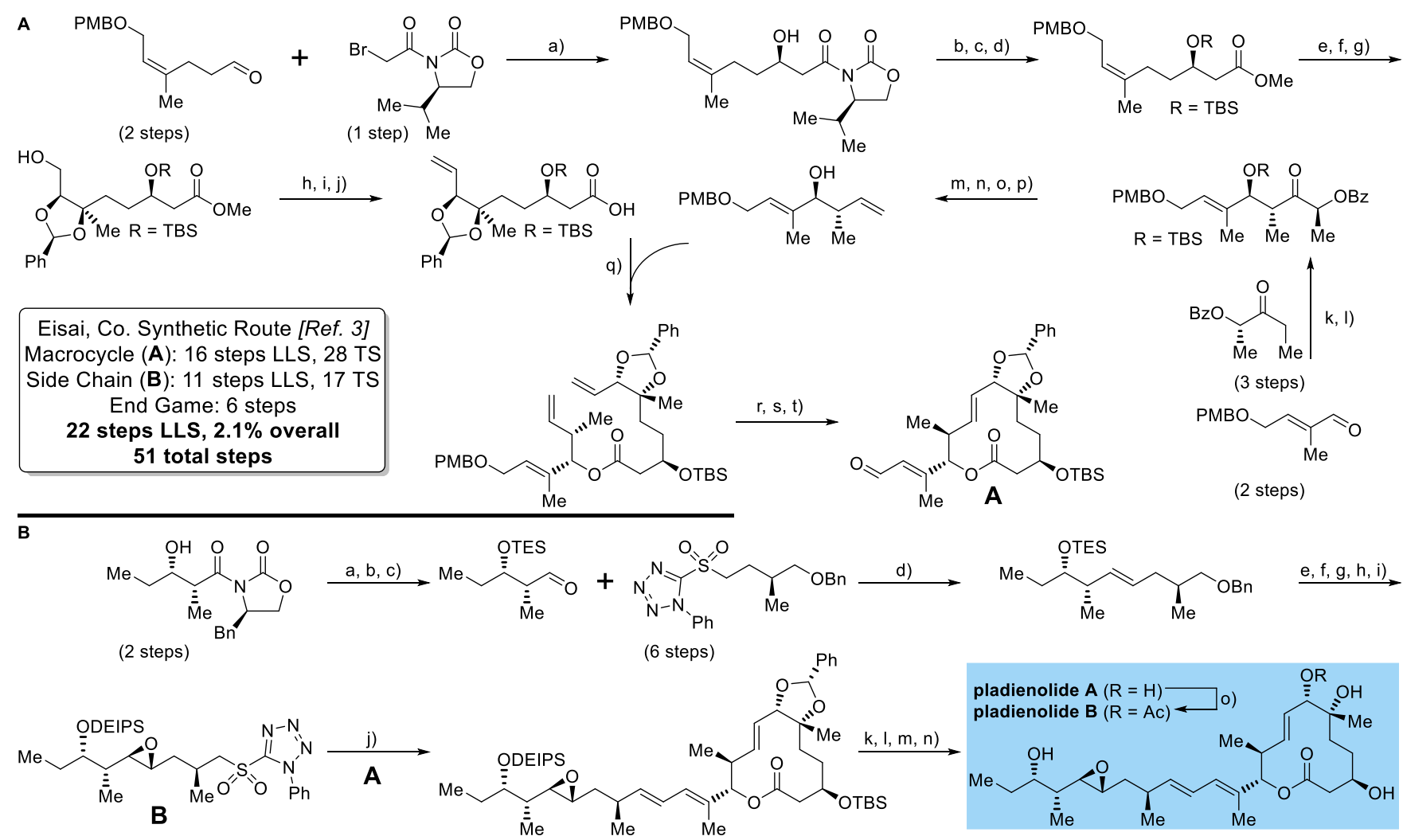

Scheme S1. Summary of the total syntheses of pladienolides A (1) and B (2) by Y. Kotake, et al. (Eisai, Co.). ${ }^{3}$ (A) Preparation of macrocyclic fragment A. Reagents and conditions: (a) $\mathrm{Sm}^{0}, \mathrm{CH}_{2} \mathrm{I}_{2}(90 \%)$; (b) $\mathrm{LiOH}, \mathrm{H}_{2} \mathrm{O}_{2}$; (c) TMS diazomethane; (d) TBSCl (85\% 3 steps); (e) AD-mix- $\alpha$, methanesulfonamide (92\%); (f) PPTS, benzaldehyde dimethyl acetal (98\%); (g) DDQ (65\%); (h) DMP; (i) methyltriphenylphosphonium iodide, $n$-BuLi (78\% 2 steps); (j) $\mathrm{LiOH}(82 \%)$; (k) $\mathrm{Cy}_{2} \mathrm{BCl}, \mathrm{Me}_{2} \mathrm{Net}(81 \%)$; (1) TBSOTf (quant); (m) $\mathrm{LiBH}_{4}$; (n) $\mathrm{NaIO}_{4}$; (o) methyltriphenylphosphonium iodide, $n$-BuLi (73\% 3 steps); (p) $1 \mathrm{~N} \mathrm{HCl} \mathrm{(99 \% );} \mathrm{(q)} \mathrm{DMAP,} \mathrm{2,4,6-trichlorobenzoyl} \mathrm{chloride,} \mathrm{Et} 3$ N (93\%); (r) Hoveyda-Grubbs II cat., BHT (46\%); (s) DDQ (80\%); (t) DMP (quant). (B) Preparation of side chain fragment B and end game. Reagents and conditions: (a) $\mathrm{N}, \mathrm{O}$-dimethylhydroxylamine hydrochloride, $\mathrm{AlMe}_{3}$ (quant); (b) TESOTf (99\%); (c) DIBAL (89\%); (d) KHMDS (68\%); (e) Li ${ }^{0}, 4,4^{\prime}$-di-tert-butyl-biphenyl (77\%); (f) 5mercapto-1-phenyltetrazole, $\mathrm{PPh}_{3}$, DIAD (91\%); (g) $\mathrm{MoO}_{7}\left(\mathrm{NH}_{4}\right)_{6} \cdot \mathrm{H}_{2} \mathrm{O}, \mathrm{H}_{2} \mathrm{O}_{2}(81 \%)$; (h) 1,2:4,5-di-Oisopropylidene-D-erythro-2,3-hexodiuro-2,6-pyranose, Oxone, $\mathrm{K}_{2} \mathrm{CO}_{3}$ (71\%); (i) DEIPSCl (quant); (j) fragment A, KHMDS (64\%); (k) TBAF (quant); (l) dichloroacetic anhydride, Et ${ }_{3} \mathrm{~N}$, DMAP (quant); (m) PPTS (64\%); (n) $\mathrm{K}_{2} \mathrm{CO}_{3}$, MeOH (96\%); (o) acetic anhydride, $\mathrm{Et}_{3} \mathrm{~N}$, DMAP (82\%). Abbreviations: BHT = 2,6-di-tert-butyl-4-methylphenol; $\mathrm{Bn}=$ benzyl; $\mathrm{Bz}=$ benzoyl; $\mathrm{Cy}=$ cyclohexyl; DDQ = 2,3-dichloro5,6-dicyano-1,4-benzoquinone; DEIPS = diethylisopropylsilyl; DIAD = diisopropyl azodicarboxylate; DIBAL = diisobutylaluminum hydride; DMAP = 4-dimethylaminopyridine; DMP $=$ Dess-Martin periodinane; KHMDS = potassium hexamethyldisilazide; Oxone $=2 \mathrm{KHSO}_{5} \cdot \mathrm{KHSO}_{4} \cdot \mathrm{K}_{2} \mathrm{SO}_{4} ; \mathrm{PMB}=p$ methoxybenzyl; PPTS $=$ pyridinium $p$-toluenesulfonate; quant = quantitative; $\mathrm{TBAF}=$ tetrabutylammonium fluoride; $\mathrm{TBS}=$ tert-butyldimethylsilyl; $\mathrm{TES}=$ triethylsilyl; $\mathrm{Tf}=$ trifluoromethanesulfonyl; TMS = trimethylsilyl. 


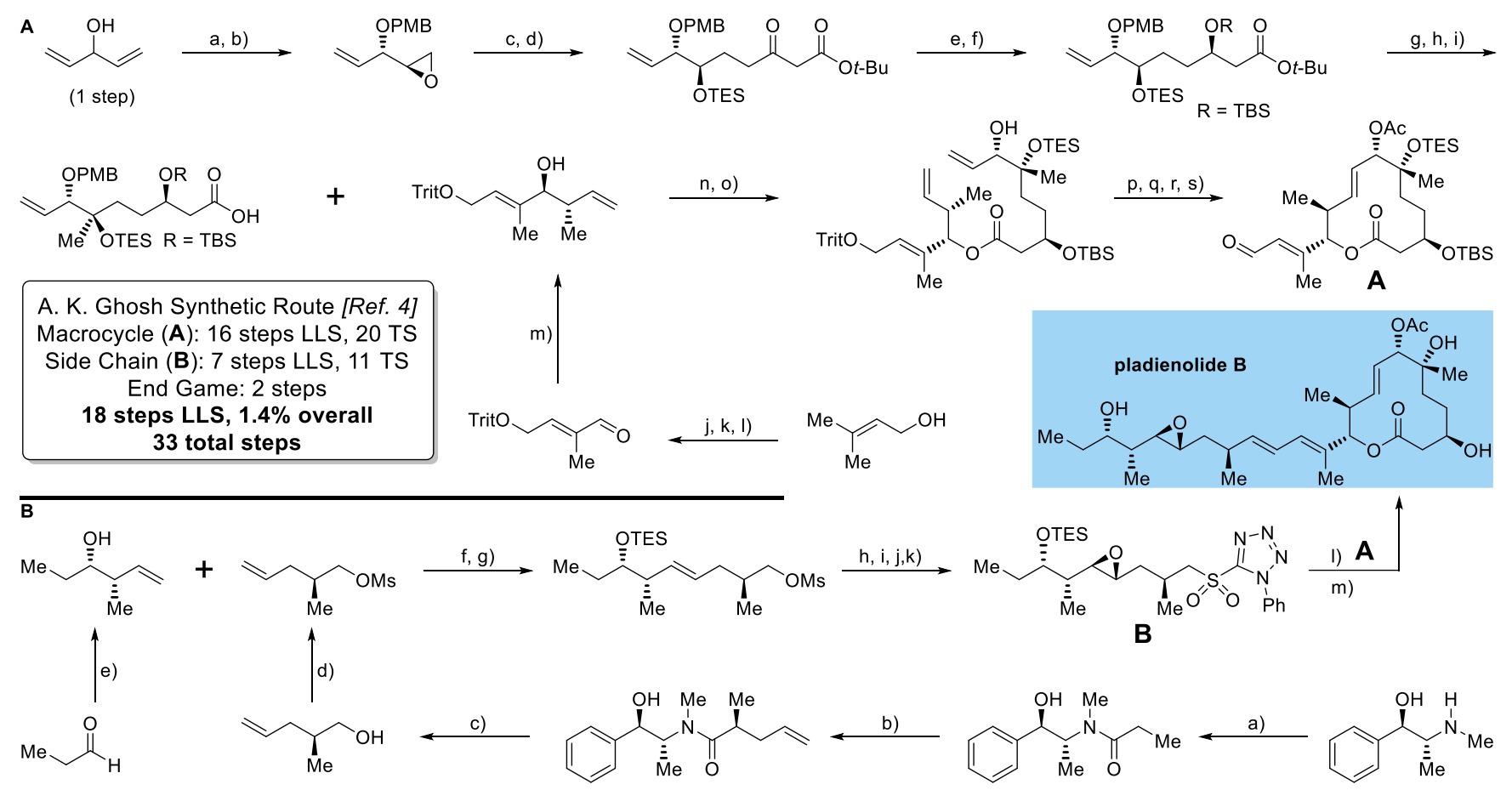

Scheme S2. Summary of the total synthesis of pladienolide B (2) by A. K. Ghosh, et al. ${ }^{4}$ (A) Preparation of macrocyclic fragment A. Reagents and conditions: (a) (+)-DIPT, $t$-BuOOH, Ti(O- $i$-Pr) 4 ; (b) NaH, PMBBr, TBAI (43\% 2 steps); (c) tert-butyl acetoacetate, NaH, $n$-BuLi; (d) TESCl; (e) $\mathrm{NaBH}_{4}$, L-tartaric acid; (f) TBSCl (61\% 4 steps); (g) IBX; (h) MeMgBr (51\% 2 steps); (i) TESOTf (96\%); (j) $\mathrm{Ph}_{3} \mathrm{CCl}, \mathrm{Et}_{3} \mathrm{~N}$, DMAP; (k) $\mathrm{SeO}_{2}, t$-BuOOH, salicylic acid (52\% 2 steps); (1) $\mathrm{MnO}_{2}(83 \%$ ); (m) $n$-BuLi, trans-butene, (-)$\mathrm{Ipc}_{2} \mathrm{BOMe}, t$-BuOK, $\mathrm{BF}_{3} \bullet \mathrm{OEt}_{2}(83 \%)$; (n) 2,4,6-trichlorobenzoyl chloride, DIPEA, DMAP; (o) DDQ (51\% 2 steps); (p) Grubbs II cat.; (q) $\mathrm{Ac}_{2} \mathrm{O}$, pyridine (72\% 2 steps); (r) DDQ; (s) IBX (51\% 2 steps). (B) Preparation of side chain fragment $\mathbf{B}$ and end game. Reagents and conditions: (a) propionyl chloride, $\mathrm{Et}_{3} \mathrm{~N}$ (quant); (b) LDA, LiCl; then allyl iodide; (c) lithium amidotrihydroborate (79\% 2 steps); (d) $\mathrm{MsCl}$ (79\%); (e) $t$-BuOK, cis-2-butene; then $n$ - $\mathrm{BuLi}$; then $\mathrm{B}(\mathrm{O} i \text {-Pr) })_{3}, 10 \% \mathrm{HCl},(-)$-DIPT; then propionaldehyde $(67 \%)$; (f) Grubbs II cat., CuI; (g) $\mathrm{TESCl}$ (47\% 2 steps); (h) NaH, 5-mercapto-1-phenyltetrazole; (i) $\mathrm{MoO}_{7}\left(\mathrm{NH}_{4}\right)_{6} \cdot \mathrm{H}_{2} \mathrm{O}, \mathrm{H}_{2} \mathrm{O}_{2}$ (71\% 2 steps); (j) 1,2:4,5-di-O-isopropylidene-D-erythro-2,3-hexodiuro-2,6pyranose, Oxone, $\mathrm{K}_{2} \mathrm{CO}_{3}$; (k) TESCl (81\% 2 steps); (1) fragment A, KHMDS; (m) TBAF (67\% 2 steps). Abbreviations: $\quad \mathrm{DDQ}=$ 2,3-dichloro-5,6-dicyano-1,4-benzoquinone; $\mathrm{DIPEA}=\mathrm{N}, \mathrm{N}-$ diisopropylethylamine; DIPT $=$ diisopropyl tartrate; DMAP = 4-dimethylaminopyridine; IBX $=2$ iodoxybenzoic acid; Ipc = isopinocampheyl; KHMDS = potassium hexamethyldisilazide; LDA = lithium diisopropylamide; $\mathrm{Ms}=$ methanesulfonyl; Oxone $=2 \mathrm{KHSO}_{5} \cdot \mathrm{KHSO}_{4} \cdot \mathrm{K}_{2} \mathrm{SO}_{4} ; \mathrm{PMB}=p$-methoxybenzyl; PPTS = pyridinium $p$-toluenesulfonate; quant = quantitative; TBAF = tetrabutylammonium fluoride; TBAI $=n$-tetrabutylammonium iodide; TBS $=$ tert-butyldimethylsilyl; TES = triethylsilyl; Tf $=$ trifluoromethanesulfonyl; TMS = trimethylsilyl; Trit = triphenylmethyl. 

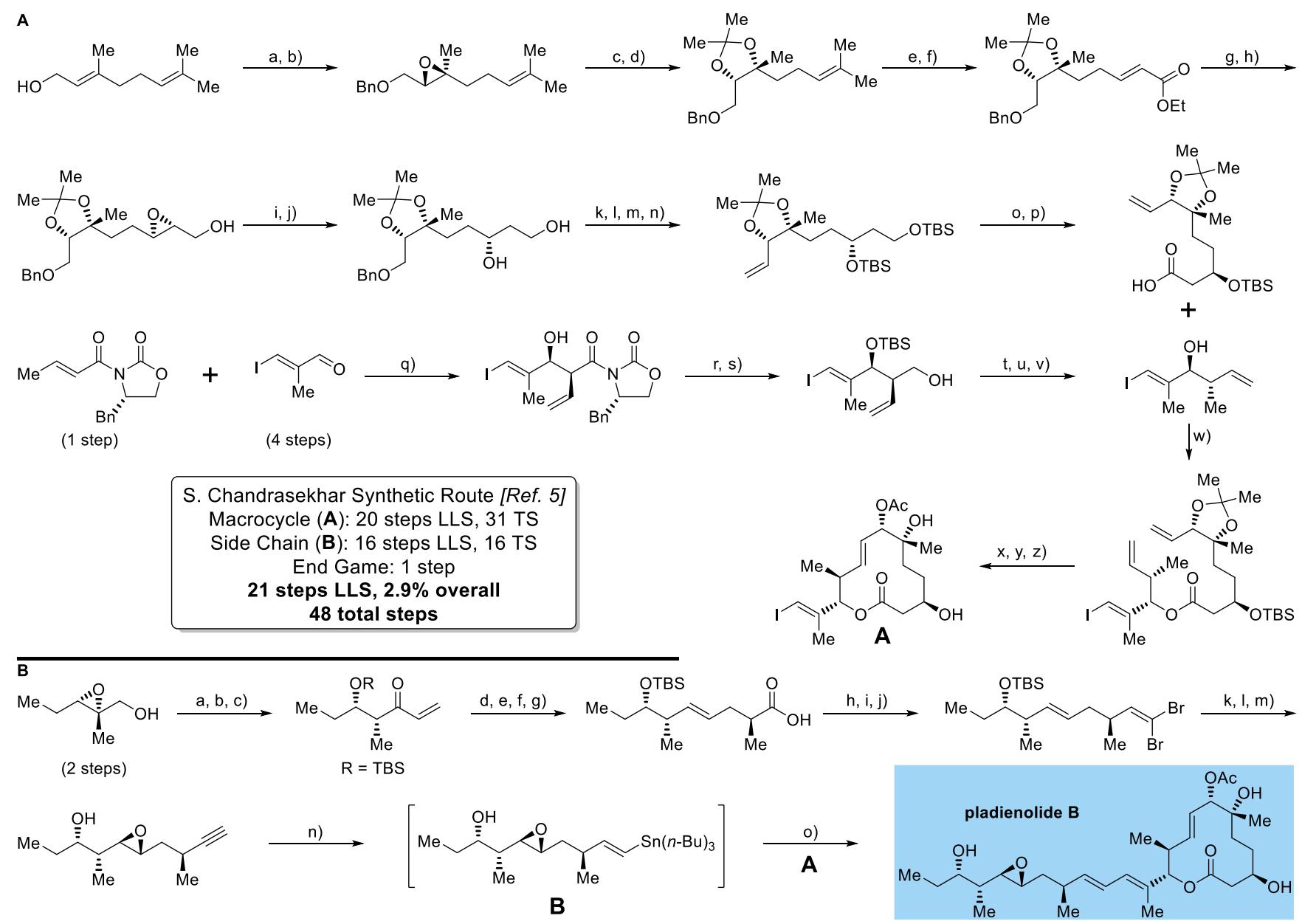

Scheme S3. Summary of the total synthesis of pladienolide B (2) by S. Chandrasekhar, et al. ${ }^{5}$ (A) Preparation of macrocyclic fragment A. Reagents and conditions: (a) (+)-DET, $t$-BuOOH, Ti(O- $i$ - $\mathrm{Pr})_{4}$ (86\%); (b) $\mathrm{NaH}, \mathrm{BnBr}(92 \%)$; (c) $\mathrm{HClO}_{4}$ (82\%); (d) CSA, 2,2-dimethoxypropane (95\%); (e) $\mathrm{O}_{3}, \mathrm{PPh}_{3}$ (86\%); (f) $\mathrm{Ph}_{3} \mathrm{P}=\mathrm{CHCO}_{2} \mathrm{Et}$ (94\%); (g) DIBAL (78\%); (h) (-)-DET, Ti(O-i-Pr) ${ }_{4}$ (82\%); (i) Red-Al; (j) $\mathrm{NaIO}_{4} \quad\left(84 \% \quad 2\right.$ steps); (k) TBSCl (92\%); (l) $\mathrm{H}_{2}$, Raney-Ni (95\%); (m) DMP; (n) methyltriphenylphosphonium bromide, $n$-BuLi (78\% 2 steps); (o) HF•py (92\%); (p) BAIB, TEMPO (83\%); (q) $n$-Bu $\mathrm{Bu}_{2} \mathrm{BOTf}, \mathrm{Et}_{3} \mathrm{~N}$ (72\%); (r) TBSOTf (92\%); (s) $\mathrm{LiBH}_{4}(74 \%)$; (t) $\mathrm{Ts}_{2} \mathrm{O}$; (u) $\mathrm{LiAlH}_{4}(80 \% 2$ steps); (v) TBAF (96\%); (w) 2,4,6-trichlorobenzoyl chloride, Et 3 N, DMAP (74\%); (x) PPTS (88\%); (y) Hoveyda-Grubbs II cat. (52\%); (z) $\mathrm{Ac}_{2} \mathrm{O}(90 \%)$. (B) Preparation of side chain fragment $\mathbf{B}$ and end game. Reagents and conditions: (a) TBSOTf (78\%); (b) vinylmagnesium bromide; (c) $\mathrm{MnO}_{2}$ (81\% 2 steps); (d) (S)-CBS, $\mathrm{BH}_{3} \cdot \mathrm{SMe}_{2}(82 \%)$; (e) (EtOC) $)_{2} \mathrm{O}, \mathrm{Et}_{3} \mathrm{~N}$ (95\%); (f) LDA, HMPA, TBSCl; (g) $\mathrm{K}_{2} \mathrm{CO}_{3}, \mathrm{MeOH}$ (80\% 2 steps); (h) $\mathrm{LiAlH}_{4}$ (75\%); (i) DMP; (j) $\mathrm{CBr}_{4}, \mathrm{PPh}_{3}$ (82\% 2 steps); (k) $n$-BuLi (78\%); (l) TBAF (94\%); (m) 1,2:4,5-di-O-isopropylidene-D-erythro-2,3-hexodiuro-2,6-pyranose, Oxone, $\mathrm{K}_{2} \mathrm{CO}_{3}(64 \%)$; (n) $(n-\mathrm{Bu})_{3} \mathrm{SnH}, \mathrm{Pd}\left(\mathrm{PPh}_{3}\right)_{4}$; (o) fragment $\mathbf{A}, \mathrm{Pd}_{2}(\mathrm{dba})_{3}, \mathrm{AsPh}, \mathrm{LiCl}$ (68\% 2 steps). Abbreviations: BAIB $=$ bis (acetoxy)iodobenzene; $\mathrm{Bn}=$ benzyl $; \mathrm{CBS}=$ Corey - Bakshi-Shibata reagent CSA = camphorsulfonic acid; $\mathrm{dba}=$ dibenzylideneacetone; DET = diethyl tartrate; DIBAL = diisobutylaluminum hydride; DMAP = 4-dimethylaminopyridine; DMP = Dess-Martin periodinane; HMPA = hexamethylphosphoramide; LDA $=$ lithium diisopropylamide; Oxone $=2 \mathrm{KHSO}_{5} \cdot \mathrm{KHSO}_{4} \cdot \mathrm{K}_{2} \mathrm{SO}_{4} ;$ PPTS $=$ pyridinium $p$ toluenesulfonate; py = pyridine; Red-Al = sodium bis(2-methoxyethoxy)aluminum hydride; $\mathrm{TBAF}=$ tetrabutylammonium fluoride; TBS = tert-butyldimethylsilyl; TEMPO = (2,2,6,6-tetramethylpiperidin-1yl)oxyl; Tf = trifluoromethanesulfonyl; Ts = p-toluenesulfonyl. 


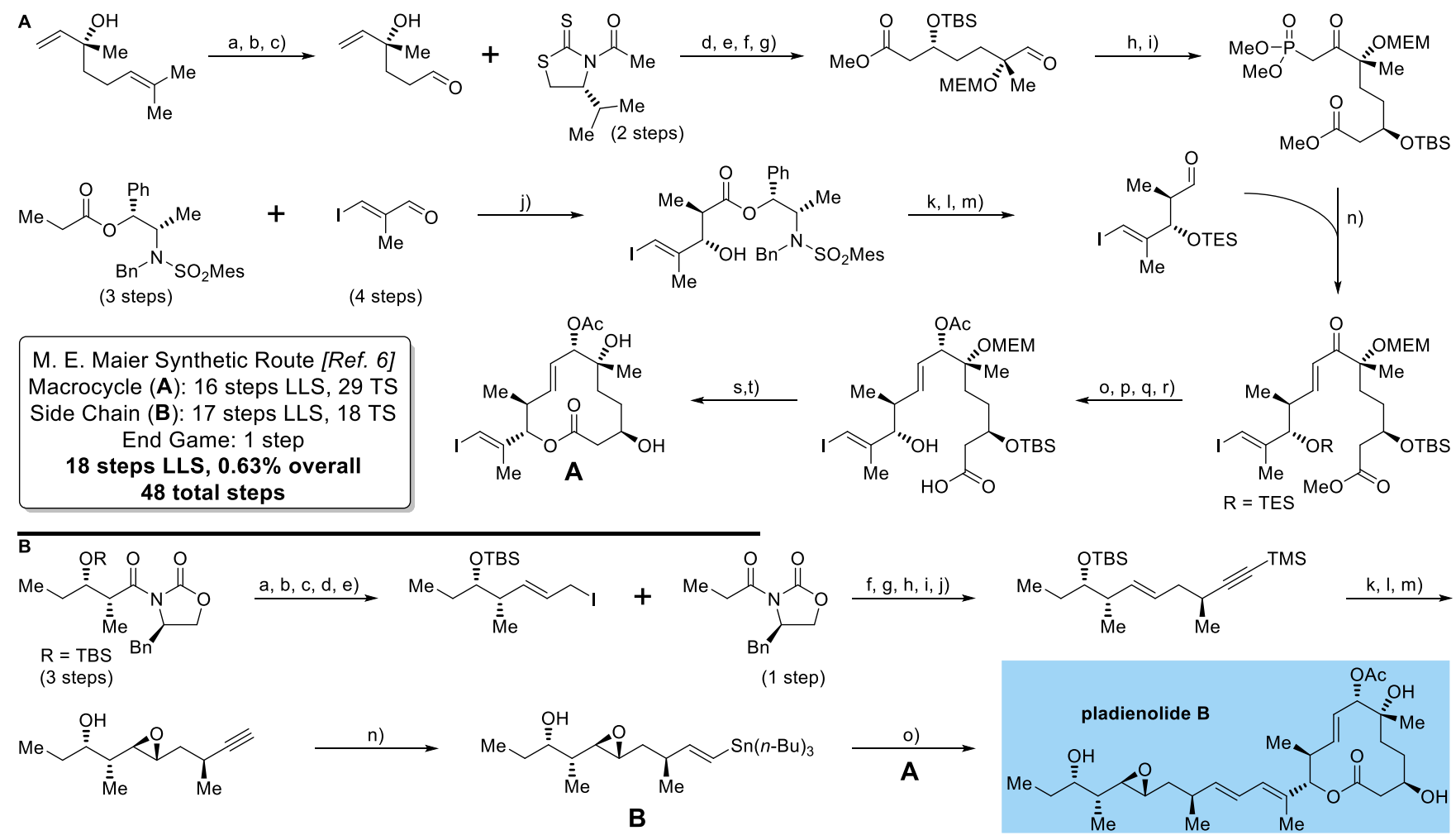

Scheme S4. Summary of the total synthesis of pladienolide B (2) by M. E. Maier, et al. ${ }^{6}$ (A) Preparation of macrocyclic fragment $A$. Reagents and conditions: (a) $\mathrm{K}_{2} \mathrm{OsO}_{2}(\mathrm{OH})_{4}, \mathrm{~K}_{3} \mathrm{Fe}(\mathrm{CN})_{6}, \mathrm{~K}_{2} \mathrm{CO}_{3}$, methanesulfonamide; (b) $\mathrm{NaIO}_{4}$; (c) $\mathrm{MEMCl}$ (65\% 3 steps); (d) $\mathrm{Sn}(\mathrm{OTf})_{2}, \mathrm{~N}$-ethylpiperidine (75\%); (e) TBSOTf; (f) $\mathrm{MeOH}$, imidazole; (g) $\mathrm{O}_{3}, \mathrm{Me}_{2} \mathrm{~S}$ (65\% 3 steps); (h) $\mathrm{MeP}(\mathrm{O})(\mathrm{OMe})_{2}, n$-BuLi (82\%); (i) DMP (86\%); (j) Cy ${ }_{2} \mathrm{BOTf}, \mathrm{Et}_{3} \mathrm{~N}$ (96\%); (k) TESCl (92\%); (l) DIBAL (86\%); (m) DMP (94\%); (n) BaO; (o) $\mathrm{ZnCl}_{2}, \mathrm{NaBH}_{4}$ (68\%); (p) $\mathrm{Ac}_{2} \mathrm{O}, \mathrm{Et}_{3} \mathrm{~N}$, DMAP (99\%); (q) $\mathrm{Me}_{3} \mathrm{SnOH}(66 \%$ ); (r) DDQ (74\%); (s) MNBA, DMAP (46\%); (t) AcOH, HCl (93\%). (B) Preparation of side chain fragment $\mathbf{B}$ and end game. Reagents and conditions: (a) $\mathrm{LiBH}_{4}(97 \%)$; (b) DMP (68\%); (c) vinylmagnesium bromide (71\%); (d) $\mathrm{SOCl}_{2}(66 \%)$; (e) $\mathrm{NaI}(80 \%)$; (f) NaHMDS (76\%); (g) $\mathrm{LiBH}_{4}(82 \%)$; (h) DMP (72\%); (i) (EtO) $2 \mathrm{P}(\mathrm{O}) \mathrm{CHN}_{2}, t$-BuOK (70\%); (j) $n$-BuLi, TMSCl (94\%); (k) PPTS (98\%); (1) 1,2:4,5-di- $O$-isopropylidene-D-erythro-2,3hexodiuro-2,6-pyranose, Oxone, $\mathrm{K}_{2} \mathrm{CO}_{3}(78 \%)$; (m) $\mathrm{K}_{2} \mathrm{CO}_{3}, \mathrm{MeOH}(86 \%)$; (n) (n-Bu) $3 \mathrm{SnH}, \mathrm{Pd}\left(\mathrm{PPh}_{3}\right)_{2} \mathrm{Cl}_{2}$ (73\%); (o) fragment $\mathbf{A}, \mathrm{Pd}_{2}(\mathrm{dba})_{3}, \mathrm{AsPh}_{3}, \mathrm{LiCl}$ (33\%). Abbreviations: $\mathrm{Bn}=$ benzyl; $\mathrm{Cy}=$ cyclohexyl; dba $=$ dibenzylideneacetone; $\mathrm{DDQ}=$ 2,3-dichloro-5,6-dicyano-1,4-benzoquinone; DIBAL = diisobutylaluminum hydride; DMAP = 4-dimethylaminopyridine; DMP = Dess-Martin periodinane; MEM = 2-methoxyethoxymethyl; Mes = 2,4,6-trimethylphenyl; MNBA = 2-methyl-6-nitrobenzoic anhydride; Oxone $=2 \mathrm{KHSO}_{5} \cdot \mathrm{KHSO}_{4} \cdot \mathrm{K}_{2} \mathrm{SO}_{4} ;$ PPTS $=$ pyridinium $p$-toluenesulfonate; $\mathrm{TBS}=$ tertbutyldimethylsilyl; TES = triethylsilyl; Tf = trifluoromethanesulfonyl; TMS $=$ trimethylsilyl. 
D. Rhoades, * et al.

\section{Summary of the Semisynthesis of H3B-8800}
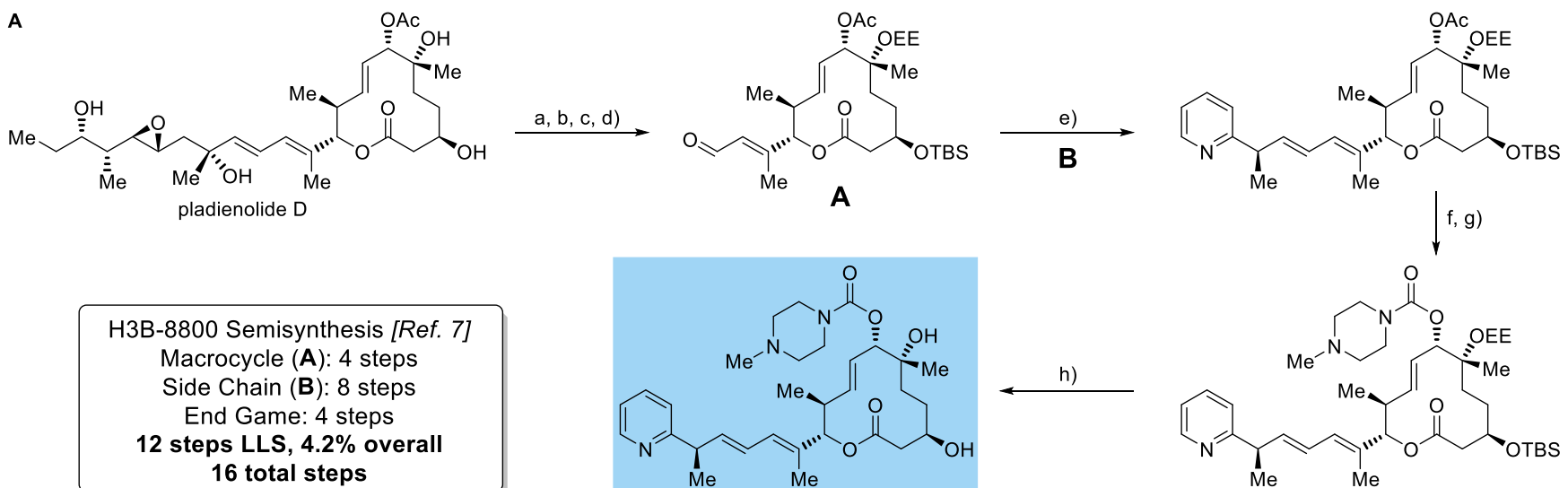

H3B-8800 Semisynthesis [Ref. 7]

Macrocycle $(\mathbf{A}): 4$ steps

End Game: 4 steps 16 total steps
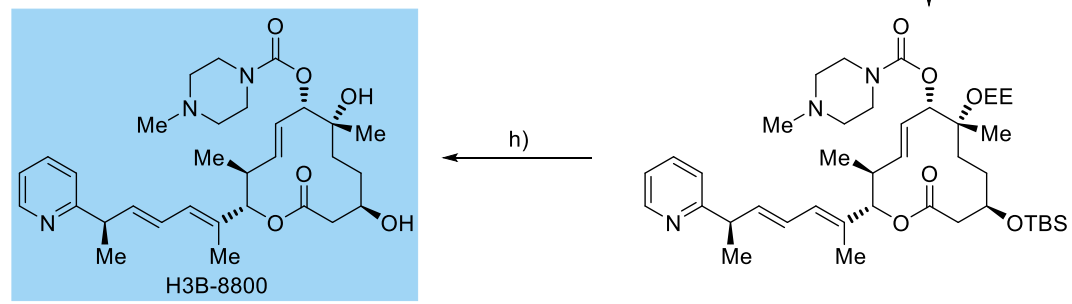

B
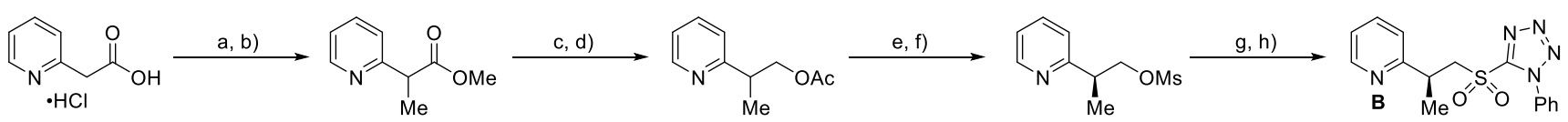

Scheme S5. Summary of the semisynthesis of H3B-8800 (3) by Eisai Co. ${ }^{7}$ (A) Preparation of macrocyclic fragment $\mathbf{A}$ from natural pladienolide $\mathbf{D}$, coupling of fragments $\mathbf{A}$ and $\mathbf{B}$, and completion of $\mathbf{3}$. Reagents and conditions: (a) TBSCl (99\%); (b) $\mathrm{OsO}_{4}$, NMO (86\%); (c) $\mathrm{Pb}(\mathrm{OAc})_{4}$ (54\%); (d) ethoxyethane, PPTS (75\%); (e) fragment B, KHMDS (53\%); (f) $\mathrm{K}_{2} \mathrm{CO}_{3}, \mathrm{MeOH}(100 \%$ ); (g) 4-nitrophenyl chloroformate; then $\mathrm{N}$-methylpiperazine (100\%); (h) PTSA (44\%). (B) Preparation of side chain fragment B. Reagents and conditions: (a) $\mathrm{SOCl}_{2}, \mathrm{MeOH}$ (95\%); (b) sodium 2-methylpropan-2-olate, MeI (91\%); (c) $\mathrm{LiAlH}_{4}(84 \%)$; (d) $\mathrm{Ac}_{2} \mathrm{O}$ (94\%); (e) porcine pancreatic lipase type II (41\%); (f) $\mathrm{MsCl}\left(98 \%\right.$ ); (g) $\mathrm{Cs}_{2} \mathrm{CO}_{3}, 1$-phenyl-1Htetrazole-5-thiol (99\%); (h) ammonium molybdate tetrahydrate, $\mathrm{H}_{2} \mathrm{O}_{2}(66 \%)$. Abbreviations: KHMDS = potassium hexamethyldisilazide; $\mathrm{Ms}=$ methanesulfonyl; $\mathrm{NMO}=N$-methylmorpholine $N$-oxide; $\mathrm{PPTS}=$ pyridinium $p$-toluenesulfonate; PTSA $=p$-toluenesulfonic acid; TBS = tert-butyldimethylsilyl. 


\section{Rhoades, * et al.}

\section{Experimental Procedures and Physical Data for Compounds}

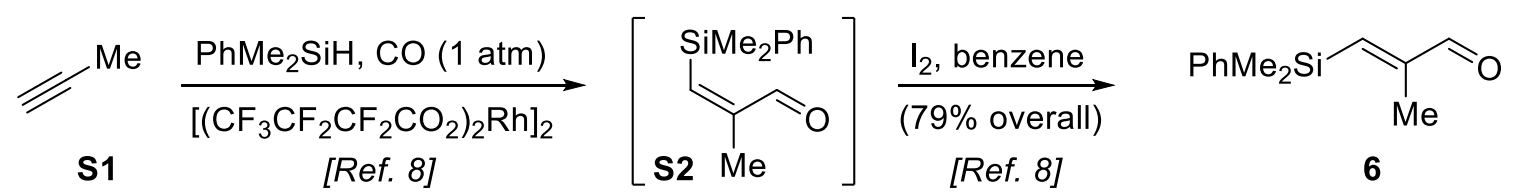

Scheme S6. Preparation of aldehyde 6 from commercially available propyne (S1).

(E)-3-[Dimethyl(phenyl)silyl]-2-methylacrylaldehyde (6): Aldehyde 6 was prepared according to a literature procedure (Scheme S6). ${ }^{8}$ To a stirred solution of dimethylphenylsilane $(7.37 \mathrm{~g}, 54.1 \mathrm{mmol}, 1.0$ equiv) in dichloromethane $(110 \mathrm{~mL})$ at $22{ }^{\circ} \mathrm{C}$ was added dirhodium(II)perfluorobutyrate (143 $\mathrm{mg}, 135$ $\mu$ mol, 0.0025 equiv). Then an atmosphere of carbon monoxide ( $1 \mathrm{~atm})$ was introduced, followed by a solution of propyne (S1) (1.0 M dichloromethane, $70.3 \mathrm{~mL}, 70.3 \mathrm{mmol}, 1.3$ equiv). After stirring for 12 h, the carbon monoxide atmosphere was carefully removed in a well-ventilated fume hood, and the reaction mixture was concentrated under reduced pressure. The obtained residue was redissolved in benzene $(250 \mathrm{~mL})$ with stirring at $22{ }^{\circ} \mathrm{C}$ in the dark, and iodine (411 $\mathrm{mg}, 1.62 \mathrm{mmol}, 0.03$ equiv) was added. After stirring for $12 \mathrm{~h}$, the reaction mixture was quenched with a saturated aqueous solution of sodium thiosulfate $(30 \mathrm{~mL})$. The two phases were separated, and the organic phase was dried over anhydrous sodium sulfate and concentrated under reduced pressure. The obtained residue was purified by flash column chromatography (silica gel, $5 \rightarrow 15 \%$ ethyl acetate in hexanes) to afford aldehyde 6 [8.72 g, $42.7 \mathrm{mmol},(E):(Z)>20: 1,79 \%$ overall yield] as a colorless oil. The physical and spectral data are consistent with those previously reported. ${ }^{9}$

$\mathbf{R}_{f}=0.35$ (5\% ethyl acetate in hexanes, UV, $p$-anisaldehyde).

FT-IR (thin film): $v_{\max }$ 3070, 3052, 3021, 2957, 2900, 2800, 2694, 1694, 1599, 1488, 1428, 1410, 1386, $1372,1332,1303,1250,1190,1150,1110,1038,1020,998,840,813,780,732,699,668 \mathrm{~cm}^{-1}$.

${ }^{1}$ H NMR (600 MHz, CDCl3): $\delta 9.46$ (s, 1 H), 7.54-7.52 (m, 2 H), 7.42-7.37 (m, 3 H), 6.84 (q, $J=0.9$ $\mathrm{Hz}, 1 \mathrm{H}), 1.81$ (br d, $J=0.9 \mathrm{~Hz}, 3 \mathrm{H}), 0.50$ (s, $6 \mathrm{H}) \mathrm{ppm}$.

${ }^{13}$ C NMR (151 MHz, CDCl3): $\delta$ 196.0, 153.5, 151.3, 137.1, 133.8, 129.7, 128.3, 13.8, -1.8 ppm.

HRMS (CI, $\boldsymbol{m} / z)$ : calcd for $\mathrm{C}_{12} \mathrm{H}_{15} \mathrm{OSi}^{+}\left[\mathrm{M}-\mathrm{H}^{-}\right]^{+}$203.0887, found 203.0888, $\Delta 0.5 \mathrm{ppm}$. 


\section{Rhoades, * et al.}

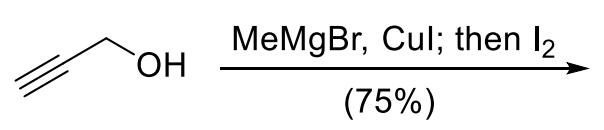

S3

[Ref. 10]

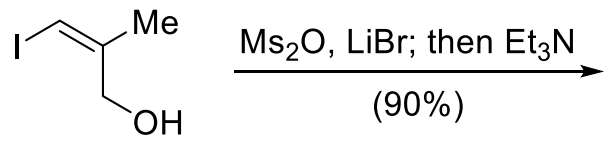

S4

[Ref. 11]

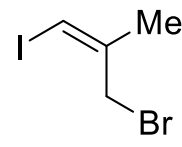

8

Scheme S7. Preparation of allyl bromide 8 from commercially available propargyl alcohol (S3).

(Z)-3-Iodo-2-methylprop-2-en-1-ol (S4): Allylic alcohol S4 was prepared according to a literature procedure (Scheme S7) ${ }^{10}$ To a stirred suspension of copper(I) iodide (1.82 g, $9.56 \mathrm{mmol}, 0.1$ equiv) in tetrahydrofuran $(86 \mathrm{~mL})$ at $0{ }^{\circ} \mathrm{C}$ was added propargyl alcohol $(5.20 \mathrm{~mL}, 95.6 \mathrm{mmol}, 1.0$ equiv) followed by the dropwise addition of a solution of methylmagnesium bromide (3.0 M diethyl ether, $63.7 \mathrm{~mL}, 191$ $\mathrm{mmol}, 2.0$ equiv). After stirring for $1.5 \mathrm{~h}$ at the same temperature, a solution of iodine ( $36.4 \mathrm{~g}, 143 \mathrm{mmol}$, 1.5 equiv) in diethyl ether $(120 \mathrm{~mL})$ was added dropwise via cannula, and the reaction mixture was stirred for an additional $1.5 \mathrm{~h}$. Then the reaction mixture was quenched with a saturated aqueous solution of ammonium chloride $(120 \mathrm{~mL})$ and a saturated aqueous solution of sodium thiosulfate $(80 \mathrm{~mL})$, diluted with water $(80 \mathrm{~mL})$, and allowed to warm to $22{ }^{\circ} \mathrm{C}$. The two phases were separated, and the aqueous layer was extracted with diethyl ether $(3 \times 15 \mathrm{~mL})$. The combined organic phases were washed with brine $(50$ $\mathrm{mL}$ ), dried over anhydrous sodium sulfate, and concentrated under reduced pressure. The obtained residue was purified by flash column chromatography (silica gel, $5 \rightarrow 20 \%$ ethyl acetate in hexanes) to afford allylic alcohol S4 [14.2 g, $71.7 \mathrm{mmol},(Z):(E)>20: 1,75 \%$ yield] as a yellow oil. The physical and spectral data are consistent with those previously reported. ${ }^{10}$ $\mathbf{R}_{f}=0.30$ (silica gel, 20\% ethyl acetate in hexanes, UV, potassium permanganate).

FT-IR (thin film): $v_{\max } 3307,3053,2946,2913,2865,1617,1435,1373,1279,1228,1134,1034,1011$, $943,826,772,665 \mathrm{~cm}^{-1}$.

${ }^{1} \mathrm{H}$ NMR (600 MHz, CDCl$): \delta 5.98(\mathrm{br} \mathrm{dq}, J=1.5,0.7 \mathrm{~Hz}, 1 \mathrm{H}), 4.25(\mathrm{br} \mathrm{d}, J=6.0 \mathrm{~Hz}, 2 \mathrm{H}), 1.98(\mathrm{~d}, J$ $=1.5 \mathrm{~Hz}, 3 \mathrm{H}), 1.60(\mathrm{br} \mathrm{d}, J=6.0 \mathrm{~Hz}, 1 \mathrm{H}) \mathrm{ppm}$.

${ }^{13}$ C NMR (151 MHz, CDCl3): $\delta$ 146.2, 75.1, 68.3, 21.8 ppm.

HRMS (EI, $\boldsymbol{m} / z$ ): calcd for $\mathrm{C}_{4} \mathrm{H}_{7} \mathrm{IO}^{*+}[\mathrm{M} \cdot]^{+} 197.9536$, found 197.9541, $\Delta 2.5 \mathrm{ppm}$. 


\section{Rhoades, * et al.}

(Z)-3-Bromo-1-iodo-2-methylprop-1-ene (8): Allylic bromide 8 was prepared from alcohol $\mathbf{S 4}$ according to a literature procedure (Scheme S7). ${ }^{11}$ To a stirred, slightly pink suspension of methanesulfonic anhydride ( $6.88 \mathrm{~g}, 39.5 \mathrm{mmol}, 1.3$ equiv) and lithium bromide (10.1 g, $116 \mathrm{mmol}, 3.8$ equiv) in diethyl ether $(65 \mathrm{~mL})$ at $-65^{\circ} \mathrm{C}$ was added a solution of allylic alcohol $\mathbf{S 4}(6.00 \mathrm{~g}, 30.4 \mathrm{mmol}$, 1.0 equiv) in diethyl ether $(10 \mathrm{~mL})$ dropwise via cannula, and the original flask was rinsed with additional diethyl ether $(3 \times 1 \mathrm{~mL})$. Then triethylamine $(6.36 \mathrm{~mL}, 45.6 \mathrm{mmol}, 1.5$ equiv $)$ was added dropwise, and the color of the suspension changed to off-white. After stirring for an additional $15 \mathrm{~min}$ at $-65^{\circ} \mathrm{C}$, the reaction mixture was allowed to warm to $0{ }^{\circ} \mathrm{C}$, and stirring was continued for $1.5 \mathrm{~h}$. Then the reaction mixture was quenched with a saturated aqueous solution of ammonium chloride $(20 \mathrm{~mL})$, diluted with water $\left(20 \mathrm{~mL}\right.$ ), and allowed to warm to $22^{\circ} \mathrm{C}$. The two phases were separated, and the aqueous layer was extracted with diethyl ether $(3 \times 15 \mathrm{~mL})$. The combined organic phases were washed with brine $(25 \mathrm{~mL})$, dried over anhydrous sodium sulfate, and concentrated under reduced pressure. Filtration of the crude residue over a short silica plug (pentanes, $200 \mathrm{~mL}$ ) and concentration of the filtrate under reduced pressure afforded pure allylic bromide $\mathbf{8}(7.12 \mathrm{~g}, 27.3 \mathrm{mmol}, 90 \%$ yield) as an air and light sensitive colorless oil that was used immediately in the following step. The physical and spectral data are consistent with those previously reported. ${ }^{12}$

$\mathbf{R}_{f}=0.59$ (silica gel, pentanes, UV, potassium permanganate).

FT-IR (thin film): $v_{\max } 3050,2944,2913,1732,1608,1560,1541,1434,1373,1286,1207,1165,1109$, $1017,909,782,734,669 \mathrm{~cm}^{-1}$.

${ }^{1} \mathbf{H}$ NMR (600 MHz, CDCl $): \delta 6.20(\mathrm{q}, J=1.3 \mathrm{~Hz}, 1 \mathrm{H}), 4.08(\mathrm{br} \mathrm{s}, 2 \mathrm{H}), 2.05(\mathrm{~d}, J=1.3 \mathrm{~Hz}, 3 \mathrm{H}) \mathrm{ppm}$.

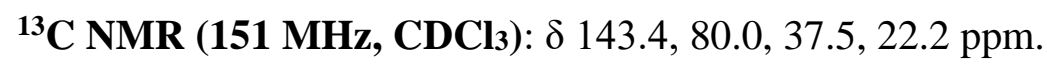

HRMS (EI, $\boldsymbol{m} / z$ ): calcd for $\mathrm{C}_{4} \mathrm{H}_{6} \mathrm{BrI}^{\cdot+}[\mathrm{M} \cdot]^{+} 259.8692$ found $259.8705, \Delta 5.0 \mathrm{ppm}$. 


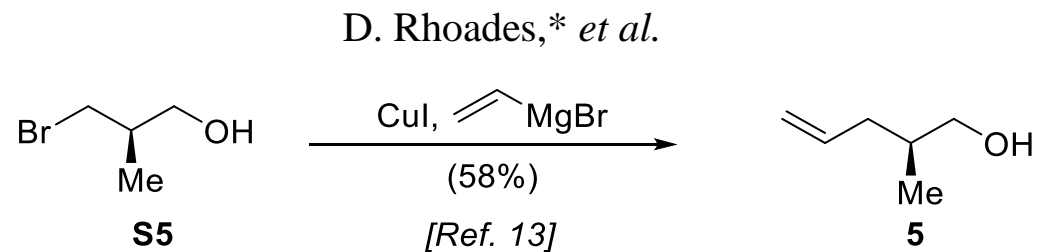

Scheme S8. Preparation of alcohol 5 from commercially available ( $R$ )-3-bromo-2-methyl-1-propanol (S5).

(S)-2-Methylpent-4-en-1-ol (5): Alcohol 5 was prepared according to a literature procedure (Scheme S8) ${ }^{13}$ To a stirred solution of copper(I) iodide $(6.44 \mathrm{~g}, 33.8 \mathrm{mmol}, 1.05$ equiv) in diethyl ether $(240 \mathrm{~mL})$ at $0{ }^{\circ} \mathrm{C}$ was added a solution of vinylmagnesium bromide $(1.0 \mathrm{M}$ tetrahydrofuran, $96 \mathrm{~mL}, 96 \mathrm{mmol}, 3.0$ equiv) dropwise via syringe pump over 45 min, forming a black suspension. After stirring for an additional $30 \mathrm{~min}$ at $0{ }^{\circ} \mathrm{C}$, a solution of $(R)$-3-bromo-2-methyl-1-propanol (S5) (4.91 g, $32.1 \mathrm{mmol}, 1.0$ equiv) in diethyl ether $(15 \mathrm{~mL})$ was added dropwise via cannula, the original flask was rinsed with additional diethyl ether $(3 \times 2 \mathrm{~mL})$, and the reaction mixture was allowed to slowly warm to $22^{\circ} \mathrm{C}$. After stirring for $12 \mathrm{~h}$, the reaction mixture was quenched with a saturated aqueous solution of ammonium chloride (100 mL). The precipitated salts were filtered and rinsed with additional diethyl ether $(150 \mathrm{~mL})$ and water $(150 \mathrm{~mL})$. The two phases were separated, and the aqueous layer was extracted with diethyl ether $(3 \times 50 \mathrm{~mL})$. The combined organic phases were washed with brine $(100 \mathrm{~mL})$, dried over anhydrous sodium sulfate, and concentrated under atmospheric pressure (water bath temperature $=50-60{ }^{\circ} \mathrm{C}$ ). The obtained residue was purified by flash column chromatography (silica gel, $15 \rightarrow 50 \%$ diethyl ether in pentanes) to afford alcohol 5 (1.86 g, $18.6 \mathrm{mmol}, 58 \%$ yield) as a colorless oil. The physical and spectral data are consistent with those previously reported..$^{14}$

$\mathbf{R}_{f}=0.50$ (silica gel, $50 \%$ diethyl ether in hexanes, potassium permanganate).

$[\boldsymbol{\alpha}]_{\mathbf{D}}^{22}=-2.8\left(c=1.00, \mathrm{CHCl}_{3}\right)$.

FT-IR (thin film): $v_{\max } 3352,3077,2957,2926,2876,1640,1554,1457,1439,1379,1270,1215,1160$, $1041,993,910,835,784,759,733,667 \mathrm{~cm}^{-1}$.

${ }^{1}$ H NMR (600 MHz, CDCl3): $\delta 5.81$ (dddd, $\left.J=17.2,10.1,7.2,7.2 \mathrm{~Hz}, 1 \mathrm{H}\right), 5.05$ (dddd, $J=17.2,1.8$, 1.2, $1.2 \mathrm{~Hz}, 1 \mathrm{H}), 5.01$ (dddd, $J=10.1,2.2,1.3,1.1 \mathrm{~Hz}, 1 \mathrm{H}), 3.51$ (dd, $J=10.6,5.8 \mathrm{~Hz}, 1 \mathrm{H}), 3.46$ (dd, 


\section{Rhoades, * et al.}

$J=10.6,5.8 \mathrm{~Hz}, 1 \mathrm{H}), 2.20-2.15$ (m, $1 \mathrm{H}), 1.97-1.92$ (m, $1 \mathrm{H}), 1.77-1.71$ (m, $1 \mathrm{H}), 1.42$ (br s, $1 \mathrm{H}), 0.92$

(d $J=6.8 \mathrm{~Hz}, 3 \mathrm{H})$ ppm.

${ }^{13}$ C NMR (151 MHz, CDCl 3$): \delta 137.1,116.2,68.1,38.0,35.7,16.5$ ppm.

HRMS (CI, $\boldsymbol{m} / z)$ : calcd for $\mathrm{C}_{6} \mathrm{H}_{13} \mathrm{O}^{+}[\mathrm{M}+\mathrm{H}]^{+}$101.0961, found 101.0959, $\Delta 2.0 \mathrm{ppm}$.

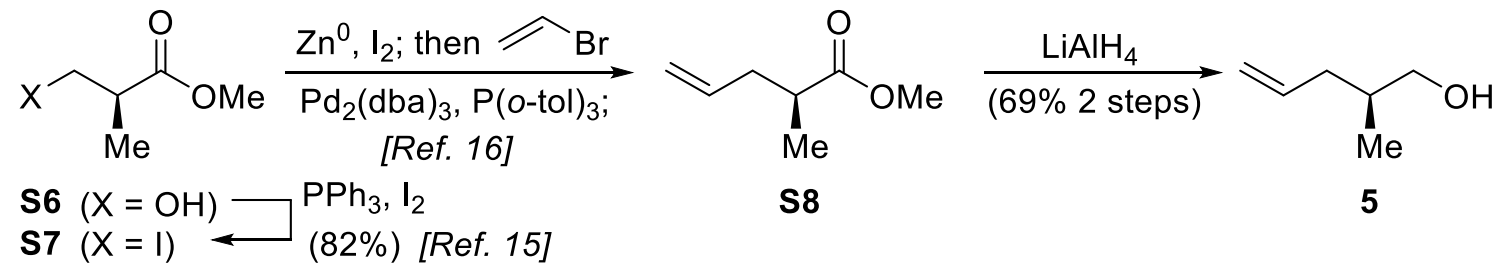

Scheme S9. Alternative preparation of alcohol 5 from commercially available $(S)$-Roche ester (S6).

Methyl $(R)$-3-iodo-2-methylpropanoate (S7): Iodide S7 was prepared according to a literature procedure (Scheme S9). ${ }^{15}$ To a stirred solution of ester S6 (2.85 g, $24.1 \mathrm{mmol}, 1.0$ equiv) in dichloromethane (80 $\mathrm{mL}$ ) at $0{ }^{\circ} \mathrm{C}$ was added triphenylphosphine (25.3 g, $96.4 \mathrm{mmol}, 4.0$ equiv), imidazole (6.56 g, $96.4 \mathrm{mmol}$, 4.0 equiv), and iodine (18.4 g, $72.3 \mathrm{mmol}, 3.0$ equiv), and the reaction mixture was allowed to slowly warm to $22{ }^{\circ} \mathrm{C}$. After 2 hours, the reaction mixture was quenched with a saturated aqueous solution of sodium bicarbonate $(40 \mathrm{~mL})$ and a saturated aqueous solution of sodium thiosulfate $(40 \mathrm{~mL})$, and the two phases were separated. The aqueous layer was extracted with dichloromethane $(3 \times 20 \mathrm{~mL})$, and the combined organic layers were washed with brine $(25 \mathrm{~mL})$, dried over anhydrous sodium sulfate, and concentrated under reduced pressure. The obtained residue was purified by flash column chromatography (silica gel, $0 \rightarrow 3 \%$ diethyl ether in hexanes) to afford pure iodide $\mathbf{S 7}$ (4.51 g, $19.8 \mathrm{mmol}, 82 \%$ yield) as a light sensitive, pale yellow oil. The physical and spectral data are consistent with those previously reported. $^{15}$

$\mathbf{R}_{f}=0.37$ (silica gel, $10 \%$ ethyl acetate in hexanes, UV, potassium permanganate). $[\boldsymbol{\alpha}]_{\mathbf{D}}^{\mathbf{2 2}}=+22.9\left(c=1.00, \mathrm{CHCl}_{3}\right)$. 


\section{Rhoades, * et al.}

FT-IR (thin film): $v_{\max } 2978,2952,2876,2846,1732,1456,1435,1376,1360,1336,1274,1250,1209$, $1154,1116,1095,1047,1015,984,905,836,822,754 \mathrm{~cm}^{-1}$.

${ }^{1} \mathbf{H}$ NMR (600 MHz, CDCl 3$): ~ \delta 3.73(\mathrm{~s}, 3 \mathrm{H}), 3.38(\mathrm{dd}, J=9.9,6.6 \mathrm{~Hz}, 1 \mathrm{H}), 3.26(\mathrm{dd}, J=9.9,6.2 \mathrm{~Hz}, 1$ H), 2.80 (qdd, $J=7.0,6.6,6.2 \mathrm{~Hz}, 1 \mathrm{H}), 1.28(\mathrm{~d}, J=7.0 \mathrm{~Hz}, 3 \mathrm{H}) \mathrm{ppm}$.

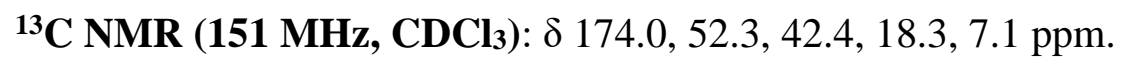

HRMS (EI, $\boldsymbol{m} / z$ ): calcd for $\mathrm{C}_{5} \mathrm{H}_{9} \mathrm{IO}_{2}{ }^{\cdot+}[\mathrm{M} \cdot]^{+}$227.9641, found 227.9640, $\Delta 0.4 \mathrm{ppm}$.

Methyl (S)-2-methylpent-4-enoate (S8): Ester S8 was prepared according to an adapted literature procedure (Scheme S9). ${ }^{16}$ A round-bottomed flask containing a stir bar and zinc dust (particle size $<10$ $\mu \mathrm{m}, 3.71 \mathrm{~g}, 56.7 \mathrm{mmol}, 3.0$ equiv) was dried under vacuum with a heat gun for $5 \mathrm{~min}$, and rigorously deoxygenated dimethylformamide (freeze-pump-thaw $\times 3,2.9 \mathrm{~mL}$ ) was added with stirring at $22{ }^{\circ} \mathrm{C}$. Iodine (721 mg. $2.84 \mathrm{mmol}, 0.15$ equiv) was added, a transient color change was observed, and the suspension became colorless again, indicating activation of the zinc metal. A solution of iodide $\mathbf{S 7}$ (4.32 $\mathrm{g}, 18.9 \mathrm{mmol}, 1.0$ equiv) in dimethylformamide $(9.5 \mathrm{~mL})$ was added dropwise via cannula, and the original flask was rinsed with additional dimethylformamide $(3 \times 1 \mathrm{~mL})$. Another portion of iodine $(721 \mathrm{mg}, 2.84$ mmol, 0.15 equiv) was added to the reaction mixture, a transient color change was observed, the suspension became colorless again, and a modest exotherm was observed. After $30 \mathrm{~min}$, tris(dibenzylideneacetone)dipalladium(0) $(865 \mathrm{mg} .94 .5 \mu \mathrm{mol}, 0.05$ equiv) and tris(o-tolyl)phosphine (575 mg. $1.89 \mathrm{mmol}, 0.10$ equiv) were added, and upon dissolution the reaction mixture was immediately cooled to $-78{ }^{\circ} \mathrm{C}$. A solution of vinyl bromide (1.0 M tetrahydrofuran, $26.5 \mathrm{~mL}, 26.5 \mathrm{mmol}, 1.4$ equiv) was added dropwise, and the reaction mixture was allowed to slowly warm to $22{ }^{\circ} \mathrm{C}$. After $12 \mathrm{~h}$, the reaction mixture was filtered through a short silica pad and rinsed thoroughly with diethyl ether $(90 \mathrm{~mL})$. The filtrate was washed with an aqueous solution of lithium chloride $(2.0 \mathrm{M}, 4 \times 15 \mathrm{~mL})$, dried over anhydrous sodium sulfate, and concentrated under atmospheric pressure (water bath temperature $=45$ $50{ }^{\circ} \mathrm{C}$ ). The crude ester $\mathbf{S 8}$ so obtained could be used directly in the following step, but could also be 


\section{Rhoades, * et al.}

purified by flash column chromatography (silica gel, $1 \rightarrow 5 \rightarrow 10 \%$ diethyl ether in pentanes) to afford ester S8 (1.77 g, $13.8 \mathrm{mmol}, 73 \%$ yield) as a colorless oil. The physical and spectral data are consistent with those previously reported. ${ }^{17}$

$\mathbf{R}_{f}=0.38$ (silica gel, 5\% diethyl ether in pentanes, potassium permanganate). $[\boldsymbol{\alpha}]_{\mathbf{D}}^{22}=+12.7\left(c=1.00, \mathrm{CHCl}_{3}\right)$.

FT-IR (thin film): $v_{\max } 3078,2926,2852,1733,1716,1675,1541,1457,1436,1379,1198,1175,1093$, $984,849,823,668 \mathrm{~cm}^{-1}$.

${ }^{1} \mathbf{H}$ NMR (600 MHz, CDCl 3 ): $\delta 5.74$ (dddd, $J=17.1,10.2,7.1,6.9$ Hz, $\left.1 \mathrm{H}\right), 5.08-5.04$ (m, $\left.1 \mathrm{H}\right), 5.04-$ $5.01(\mathrm{~m}, 1 \mathrm{H}), 3.67(\mathrm{~s}, 3 \mathrm{H}), 2.56-2.50(\mathrm{~m}, 1 \mathrm{H}), 2.43-2.38(\mathrm{~m}, 1 \mathrm{H}), 2.21-2.15(\mathrm{~m}, 1 \mathrm{H}), 1.16(\mathrm{~d}, J=7.0$ $\mathrm{Hz}, 3 \mathrm{H}) \mathrm{ppm}$.

${ }^{13}$ C NMR (151 MHz, CDCl3): $\delta$ 176.7, 135.6, 117.0, 51.7, 39.3, 38.0, 16.7 ppm. HRMS (CI, $\boldsymbol{m} / \boldsymbol{z}$ ): calcd for $\mathrm{C}_{7} \mathrm{H}_{13} \mathrm{O}_{2}[\mathrm{M}+\mathrm{H}]^{+}$129.0910, found 129.0910 .

(S)-2-Methylpent-4-en-1-ol (5): To a stirred solution of ester $\mathbf{S 8}$ (1.77 g, $13.8 \mathrm{mmol}, 1.0$ equiv) in diethyl ether $(46 \mathrm{~mL})$ at $0{ }^{\circ} \mathrm{C}$ was carefully added solid lithium aluminum hydride $(524 \mathrm{mg}, 13.8 \mathrm{mmol}, 1.0$ equiv). After stirring for an additional $1 \mathrm{~h}$, the reaction mixture was carefully quenched with sodium sulfate decahydrate ( $4.45 \mathrm{~g}, 13.8 \mathrm{mmol}, 1.0$ equiv), dried over anhydrous sodium sulfate, and concentrated under atmospheric pressure (water bath temperature $=50-60{ }^{\circ} \mathrm{C}$ ). Filtration of the obtained residue over a short silica plug (25\% pentanes in diethyl ether, $100 \mathrm{~mL})$ and concentration under atmospheric pressure (water bath temperature $\left.=50-60{ }^{\circ} \mathrm{C}\right)$ afforded alcohol $5(1.31 \mathrm{~g}, 13.1 \mathrm{mmol}, 95 \%$ yield $)$ as a colorless oil . The physical and spectral data are consistent with those previously reported. ${ }^{14}$ 
D. Rhoades, * et al.
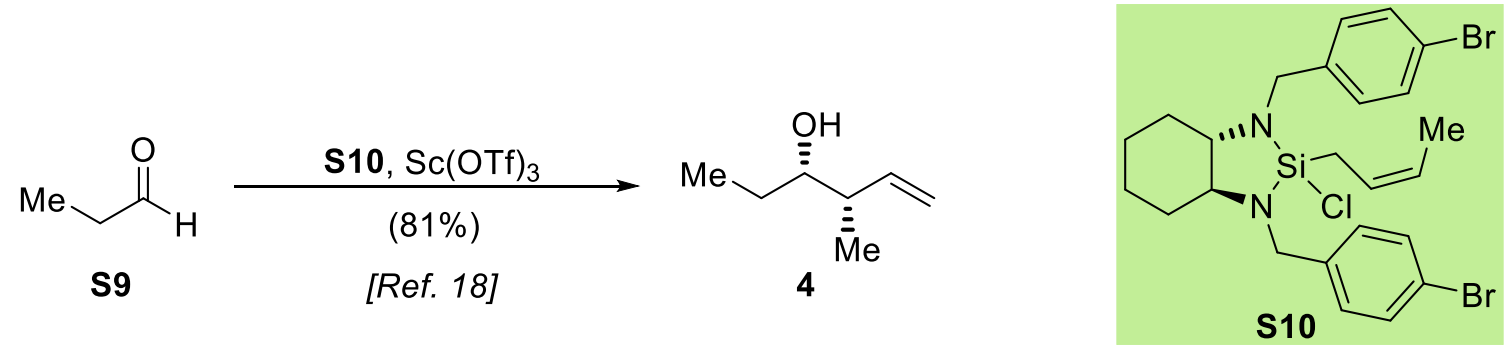

Scheme S10. Preparation of alcohol 4 from commercially available propanal (S9).

$(3 S, 4 S)$-4-Methylhex-5-en-3-ol (4): Alcohol 4 was prepared according to a literature procedure ${ }^{18}$ (Scheme S10): To a stirred solution of Leighton reagent $\mathbf{S 1 0}{ }^{19}\left(10.3 \mathrm{~g}, 18.1 \mathrm{mmol}, 1.0\right.$ equiv) at $0{ }^{\circ} \mathrm{C}$ was added scandium(III) triflate ( $312 \mathrm{mg}, 634 \mu \mathrm{mol}, 0.035$ equiv), followed by freshly distilled propanal (S9) ( $2.60 \mathrm{~mL}, 36.2 \mathrm{mmol}, 2.0$ equiv). After $3 \mathrm{~h}$, the reaction mixture was concentrated under reduced pressure, and the obtained residue was resuspended in diethyl ether $(75 \mathrm{~mL})$ with stirring at $0{ }^{\circ} \mathrm{C}$. An aqueous hydrochloric acid solution (1.0 M, $75 \mathrm{~mL})$ was added, forming a white precipitate, and the reaction mixture was allowed to slowly warm to $22{ }^{\circ} \mathrm{C}$ over $1 \mathrm{~h}$. The precipated bis-amine salt was filtered and recycled, and the filtrate was carefully neutralized with a saturated aqueous solution of sodium bicarbonate $(200 \mathrm{~mL})$. The two phases were separated, and the aqueous layer was extracted with ethyl acetate $(3 \times 30 \mathrm{~mL})$. The combined organic phases were dried over anhydrous sodium sulfate and concentrated under reduced pressure. The obtained residue was purified by flash column chromatography (silica gel, $5 \rightarrow 10 \%$ ethyl acetate in hexanes) to afford alcohol 4 (1.68 g, $14.7 \mathrm{mmol}, 81 \%$ yield) as a colorless oil. The physical and spectral data are consistent with those previously reported. ${ }^{18}$ $\mathbf{R}_{f}=0.50$ (silica gel, $20 \%$ ethyl acetate in hexanes, potassium permanganate).

$[\boldsymbol{\alpha}]_{\mathbf{D}}^{22}=-20.8\left(c=1.00, \mathrm{CHCl}_{3}\right)$.

FT-IR (thin film): $v_{\max } 3376,3018,2966,2935,2878,1487,1460,1453,1406,1374,1262,1070,1011$, $993,913,855,805,766,722,681,667 \mathrm{~cm}^{-1}$. 


\section{Rhoades, * et al.}

${ }^{1}$ H NMR (600 MHz, CDCl $): \delta 5.80(\mathrm{ddd}, J=16.8,11.0,7.4 \mathrm{~Hz}, 1 \mathrm{H}), 5.09(\mathrm{ddd}, J=16.8,1.8,1.3 \mathrm{~Hz}$, $1 \mathrm{H}), 5.07$ (ddd, $J=11.0,1.8,1.1 \mathrm{~Hz}, 1 \mathrm{H}), 3.42(\mathrm{br} \mathrm{ddd}, J=8.9,8.9,4.7 \mathrm{~Hz}, 1 \mathrm{H}), 2.32-2.26(\mathrm{~m}, 1 \mathrm{H})$, $1.59-1.52(\mathrm{~m}, 1 \mathrm{H}), 1.43-1.35(\mathrm{~m}, 2 \mathrm{H}), 1.02(\mathrm{~d}, J=6.9 \mathrm{~Hz}, 3 \mathrm{H}), 0.96(\mathrm{br} \mathrm{dd}, J=7.4,7.4 \mathrm{~Hz}, 3 \mathrm{H}) \mathrm{ppm}$. ${ }^{13}$ C NMR (151 MHz, CDCl3): $\delta$ 141.3, 115.3, 76.3, 43.2, 26.9, 14.1, 10.6 ppm.

HRMS (CI, $\boldsymbol{m} / z)$ : calcd for $\mathrm{C}_{7} \mathrm{H}_{15} \mathrm{O}^{+}[\mathrm{M}+\mathrm{H}]^{+} 115.1117$, found $115.1121, \Delta 3.5 \mathrm{ppm}$.
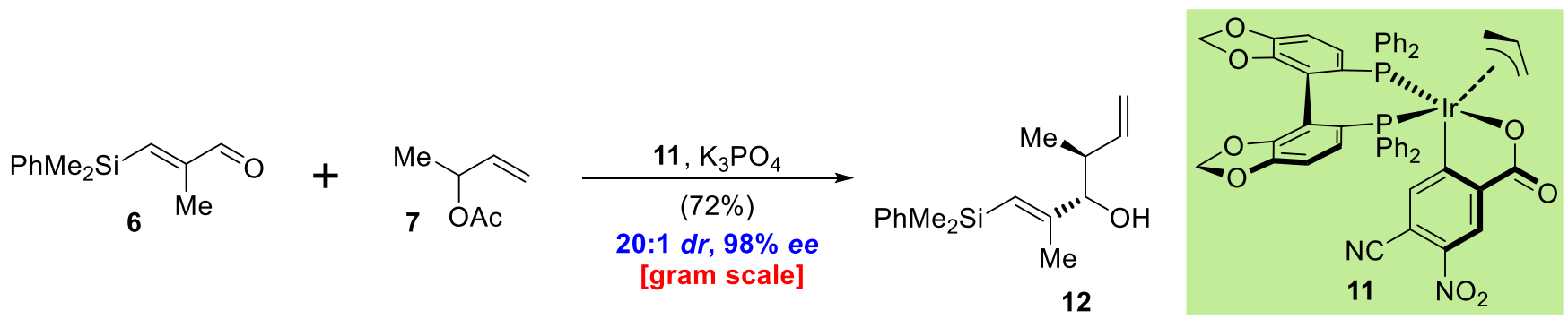

(3S,4S,E)-1-[Dimethyl(phenyl)silyl]-2,4-dimethylhexa-1,5-dien-3-ol (12): To a stirred solution of aldehyde 6 (3.17 g, $15.5 \mathrm{mmol}, 1.0$ equiv) in tetrahydrofuran $(7.7 \mathrm{~mL})$ at $22{ }^{\circ} \mathrm{C}$ was added $(S)$-Krische catalyst $\mathbf{1 1}^{20}$ ( $801 \mathrm{mg}, 0.775 \mathrm{mmol}, 0.05$ equiv), potassium phosphate $(1.65 \mathrm{~g}, 7.75 \mathrm{mmol}, 0.5$ equiv), isopropanol (2.40 mL, $31.0 \mathrm{mmol}, 2.0$ equiv), water ( $1.40 \mathrm{~mL}, 77.5 \mathrm{mmol}, 5.0$ equiv), and but-3-en-2-yl acetate (7) (3.93 mL, $31.0 \mathrm{mmol}, 2.0$ equiv). Stirring was continued for an additional $30 \mathrm{~min}$ at the same temperature, and then the reaction mixture was heated to $60{ }^{\circ} \mathrm{C}$. After $24 \mathrm{~h}$, the reaction mixture was allowed to cool to $22^{\circ} \mathrm{C}$, filtered over a pad of celite, rinsed thoroughly with ethyl acetate (50 mL), and concentrated under reduced pressure. The crude residue was purified by flash column chromatography (silica gel, $1 \rightarrow 2 \rightarrow 5 \%$ ethyl acetate in hexanes) to afford alcohol 12 (2.92 g, $11.2 \mathrm{mmol}, 72 \%$ yield) as a colorless oil.

$\mathbf{R}_{f}=0.29$ (silica gel, 10\% ethyl acetate in hexanes, UV, $p$-anisaldehyde).

$[\boldsymbol{\alpha}]_{\mathbf{D}}^{22}=-16.5\left(c=1.00, \mathrm{CH}_{2} \mathrm{Cl}_{2}\right)$.

FT-IR (thin film): $v_{\max } 3451,3069,3050,2960,2929,2871,1638,1618,1487,1454,1427,1377,1316$, $1299,1248,1190,1144,1113,1071,1013,999,968,914,833,780,729,699 \mathrm{~cm}^{-1}$. 


\section{Rhoades, * et al.}

${ }^{1} \mathbf{H}$ NMR (600 MHz, CDCl $)$ : $\delta$ 7.56-7.53 (m, $\left.2 \mathrm{H}\right), 7.36-7.34$ (m, $\left.3 \mathrm{H}\right), 5.77-5.71$ (m, $\left.1 \mathrm{H}\right), 5.62$ (br q, $J=0.7 \mathrm{~Hz}, 1 \mathrm{H}), 5.17-5.16(\mathrm{~m}, 1 \mathrm{H}), 5.15-5.14(\mathrm{~m}, 1 \mathrm{H}), 3.76(\mathrm{dd}, J=7.5,2.9 \mathrm{~Hz}, 1 \mathrm{H}), 2.35-2.29(\mathrm{~m}$, $1 \mathrm{H}), 1.82$ (br s, $1 \mathrm{H}), 1.69$ (br d, $J=0.7 \mathrm{~Hz}, 3 \mathrm{H}), 0.98$ (d, $J=6.8 \mathrm{~Hz}, 3 \mathrm{H}), 0.39$ (s, $3 \mathrm{H}), 0.38(\mathrm{~s}, 3 \mathrm{H})$ ppm.

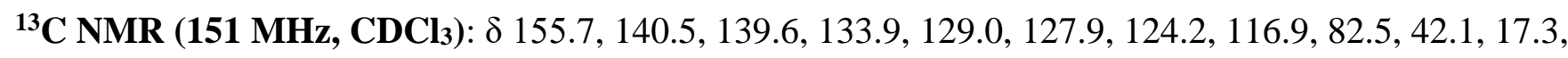
$17.0,-0.90,-1.0 \mathrm{ppm}$.

HRMS (ESI, $\boldsymbol{m} / z)$ ): calcd for $\mathrm{C}_{16} \mathrm{H}_{24} \mathrm{OSiNa}^{+}[\mathrm{M}+\mathrm{Na}]^{+} 283.1489$, found 283.1496, $\Delta 2.5 \mathrm{ppm}$.

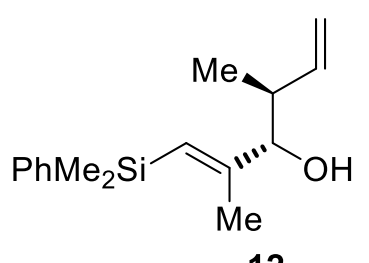

12

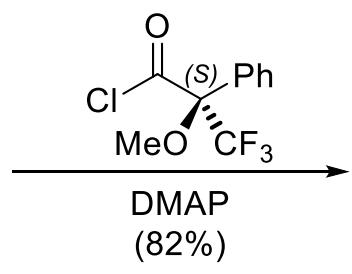

(82\%)

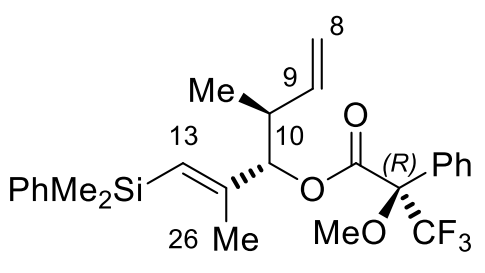

(R)-S11

\section{(3S,4S,E)-1-[Dimethyl(phenyl)silyl]-2,4-dimethylhexa-1,5-dien-3-yl $(R)$-3,3,3-trifluoro-2-methoxy-}

2-phenylpropanoate [(R)-S11]: To a stirred solution of alcohol 12 (5.8 mg, $22 \mu \mathrm{mol}, 1.0$ equiv) in dichloromethane $(1.0 \mathrm{~mL})$ at $22{ }^{\circ} \mathrm{C}$ was added $(S)-(+)$ - $\alpha$-methoxy- $\alpha$-(trifluoromethyl)phenylacetyl chloride ( $12 \mu \mathrm{L}, 66 \mu \mathrm{mol}, 3.0$ equiv), followed by 4-dimethylaminopyridine ( $11 \mathrm{mg}, 88 \mu \mathrm{mol}, 4.0$ equiv). After stirring for $4 \mathrm{~h}$, the reaction mixture was concentrated under reduced pressure and purified directly by preparative thin layer chromatography (5\% ethyl acetate in hexanes) to afford Mosher ester $(\boldsymbol{R})$-S11 (8.6 mg, $18 \mu \mathrm{mol}, 82 \%$ yield) as a colorless oil.

$\mathbf{R}_{f}=0.41$ (silica gel, 5\% ethyl acetate in hexanes, UV, $p$-anisaldehyde).

$[\boldsymbol{\alpha}]_{\mathbf{D}}^{22}=+39.0\left(c=1.00, \mathrm{CH}_{2} \mathrm{Cl}_{2}\right)$.

FT-IR (thin film): $v_{\max } 3069,2956,2932,2850,1747,1643,1619,1498,1488,1451,1428,1379,1250$, $1183,1168,1122,1113,1081,1018,994,966,946,922,856,836,816,780,729,722,699,678 \mathrm{~cm}^{-1}$. 


\section{Rhoades, * et al.}

${ }^{1}$ H NMR (600 MHz, CDCl3): $\delta$ 7.51-7.47 (m, 4 H), 7.37-7.32 (m, 4 H), 7.31-7.28 (m, 2 H), 5.72 (ddd, $J=17.3,10.2,8.1 \mathrm{~Hz}, 1 \mathrm{H}), 5.68$ (br s, $1 \mathrm{H}), 5.15(\mathrm{~d}, J=8.5 \mathrm{~Hz}, 1 \mathrm{H}), 5.09-5.04(\mathrm{~m}, 2 \mathrm{H}), 3.54(\mathrm{~s}, 3 \mathrm{H})$, 2.55-2.49 (m, $1 \mathrm{H}), 1.49$ (br s, $3 \mathrm{H}), 0.97$ (d, J = 6.9 Hz, $3 \mathrm{H}), 0.35$ (s, $3 \mathrm{H}), 0.34$ (s, $3 \mathrm{H}) \mathrm{ppm}$.

${ }^{13}$ C NMR (151 MHz, CDCl3): $\delta$ 165.9, 150.6, 139.8, 139.1, 133.8, 132.6, 129.6, 129.1, 128.3, 128.01, 127.97, 127.4, $123.5(\mathrm{q}, J=288.8 \mathrm{~Hz}), 116.3,86.7,84.7(\mathrm{q}, J=27.6 \mathrm{~Hz}), 56.0,40.0,17.7,17.0,-1.1$, $-1.2 \mathrm{ppm}$.

${ }^{19}$ F NMR (565 MHz, CDCl3): $\delta-70.3$ ppm.

HRMS (ESI, $\boldsymbol{m} / z$ ): calcd for $\mathrm{C}_{26} \mathrm{H}_{31} \mathrm{~F}_{3} \mathrm{O}_{3} \mathrm{SiNa}^{+}[\mathrm{M}+\mathrm{Na}]^{+}$499.1887, found 499.1899, $\Delta 2.4$ ppm.

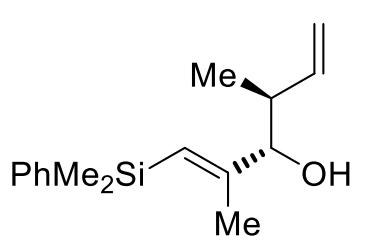

12

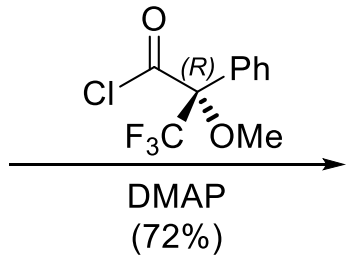

$(72 \%)$

\section{(3S,4S,E)-1-[Dimethyl(phenyl)silyl]-2,4-dimethylhexa-1,5-dien-3-yl (S)-3,3,3-trifluoro-2-methoxy-}

2-phenylpropanoate [(S)-S11]: To a stirred solution of alcohol 12 (4.8 mg, $18 \mu \mathrm{mol}, 1.0$ equiv) in dichloromethane $(1.0 \mathrm{~mL})$ at $22{ }^{\circ} \mathrm{C}$ was added $(R)-(-)$ - $\alpha$-methoxy- $\alpha$-(trifluoromethyl)phenylacetyl chloride (10 $\mu \mathrm{L}, 54 \mu \mathrm{mol}, 3.0$ equiv), followed by 4-dimethylaminopyridine ( $8.8 \mathrm{mg}, 72 \mu \mathrm{mol}, 4.0$ equiv). After stirring for $4 \mathrm{~h}$, the reaction mixture was concentrated under reduced pressure and purified directly by preparative thin layer chromatography (5\% ethyl acetate in hexanes) to afford Mosher ester $(\boldsymbol{S})$-S11 (6.3 mg, $13 \mu \mathrm{mol}, 72 \%$ yield) as a colorless oil.

$\mathbf{R}_{f}=0.41$ (silica gel, 5\% ethyl acetate in hexanes, UV, $p$-anisaldehyde). $[\boldsymbol{\alpha}]_{\mathbf{D}}^{22}=-25.3\left(c=1.00, \mathrm{CH}_{2} \mathrm{Cl}_{2}\right)$.

FT-IR (thin film): $v_{\max } 3069,2957,2850,1748,1643,1619,1497,1451,1428,1379,1290,1270,1249$, 1182, 1169, 1114, 1081, 1017, 993, 965, 920, 858, 836, 816, 780, 730, 722, 699, $677 \mathrm{~cm}^{-1}$. 


\section{Rhoades, * et al.}

${ }^{1}$ H NMR (600 MHz, CDCl $)$ ): $\delta$ 7.51-7.47 (m, 4 H), 7.39-7.31 (m, 6 H), 5.75 (br s, $\left.1 \mathrm{H}\right), 5.62$ (ddd, $J=$

17.4, 10.2, 8.1 Hz, $1 \mathrm{H}), 5.23(\mathrm{~d}, J=8.2 \mathrm{~Hz}, 1 \mathrm{H}), 4.98-4.93(\mathrm{~m}, 2 \mathrm{H}), 3.48(\mathrm{~s}, 3 \mathrm{H}), 2.52-2.46(\mathrm{~m}, 1 \mathrm{H})$, 1.67 (br s, $3 \mathrm{H}), 0.96$ (d, $J=6.9 \mathrm{~Hz}, 3 \mathrm{H}), 0.38$ (s, $3 \mathrm{H}), 0.37$ (s, $3 \mathrm{H}) \mathrm{ppm}$.

${ }^{13}$ C NMR (151 MHz, CDCl3): $\delta$ 165.9, 150.8, 139.0, 133.8, 132.6, 129.6, 129.1, 128.4, 128.1, 128.0, 127.7, $123.6(\mathrm{q}, J=288.7 \mathrm{~Hz}), 116.2,86.3,84.6(\mathrm{q}, J=27.7 \mathrm{~Hz}), 55.5,40.2,18.0,16.7,-1.1,-1.2 \mathrm{ppm}$. ${ }^{19}$ F NMR (565 MHz, CDCl3): $\delta-70.0 \mathrm{ppm}$.

HRMS (ESI, $\boldsymbol{m} / z)$ ): calcd for $\mathrm{C}_{26} \mathrm{H}_{31} \mathrm{~F}_{3} \mathrm{O}_{3} \mathrm{SiNa}^{+}[\mathrm{M}+\mathrm{Na}]^{+}$499.1887, found 499.1902, $\Delta 3.0 \mathrm{ppm}$.

Table S1. Mosher ester analysis of MTPA esters derived from alcohol 12.

\begin{tabular}{|c|c|c|c|}
\hline Proton & $\begin{array}{c}\text { Chemical shift of }(\boldsymbol{S})- \\
\text { MTPA derivative }\end{array}$ & $\begin{array}{c}\text { Chemical shift of }(\boldsymbol{R})- \\
\text { MTPA derivative }\end{array}$ & $\begin{array}{c}\Delta \boldsymbol{\delta}:(\boldsymbol{S}) \text {-MTPA }- \\
(\boldsymbol{R})-\mathrm{MTPA}\end{array}$ \\
\hline $\mathbf{1 3}$ & 5.75 & 5.68 & +0.07 \\
\hline $\mathbf{2 6}$ & 1.67 & 1.49 & +0.18 \\
\hline $\mathbf{1 0}$ & 2.49 & 2.52 & -0.03 \\
\hline $\mathbf{9}$ & 5.62 & 5.72 & -0.10 \\
\hline $\mathbf{8 a}$ & 4.96 & 5.07 & -0.11 \\
\hline $\mathbf{8 b}$ & 4.94 & 5.05 & -0.11 \\
\hline
\end{tabular}

On the basis of the Mosher ester analysis, ${ }^{21}$ the absolute configuration at C11 (pladienolide numbering) is unambiguously assigned as $(S)$.

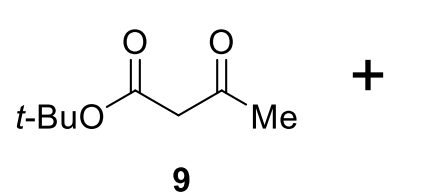

9<smiles>CC(=CI)CBr</smiles>

8

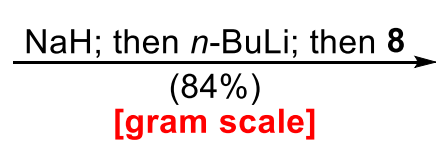

[gram scale]

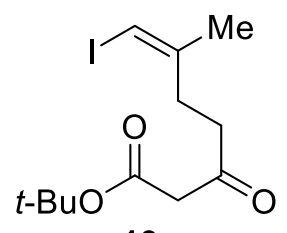

13

tert-Butyl (Z)-7-iodo-6-methyl-3-oxohept-6-enoate (13): To a stirred solution of tert-butyl acetate (4.39 g, $27.7 \mathrm{mmol}, 1.1$ equiv) in tetrahydrofuran $(139 \mathrm{~mL})$ at $0{ }^{\circ} \mathrm{C}$ was carefully added sodium hydride $(60 \%$ $w / w$ in mineral oil, $1.11 \mathrm{~g}, 27.7 \mathrm{mmol}, 1.1$ equiv) in portions. After stirring for $15 \mathrm{~min}$ at the same temperature, the reaction mixture was cooled to $-78{ }^{\circ} \mathrm{C}$, and a solution of $n$-butyllithium $(2.5 \mathrm{M}$ in hexanes, $11.1 \mathrm{~mL}, 27.7 \mathrm{mmol}, 1.1$ equiv) was added dropwise. After stirring for $15 \mathrm{~min}$ at the same temperature, a solution of bromide $8(6.57 \mathrm{~g}, 25.2 \mathrm{mmol}, 1.0$ equiv) in tetrahydrofuran (10 mL) was added 


\section{Rhoades, * et al.}

dropwise via cannula, the original flask was rinsed with additional tetrahydrofuran $(3 \times 2 \mathrm{~mL})$, and the reaction mixture was allowed to slowly warm to $0{ }^{\circ} \mathrm{C}$ over $1.5 \mathrm{~h}$. Then the reaction mixture was quenched with a saturated aqueous solution of ammonium chloride $(50 \mathrm{~mL})$, and allowed to warm to $22{ }^{\circ} \mathrm{C}$. The two phases were separated, and the aqueous layer was extracted with ethyl acetate $(3 \times 15 \mathrm{~mL})$. The combined organic phases were dried over anhydrous sodium sulfate and concentrated under reduced pressure. The obtained residue was purified by flash column chromatography (silica gel, $2 \rightarrow 5 \rightarrow 10 \%$ ethyl acetate in hexanes) to afford $\beta$-ketoester $\mathbf{1 3}(7.17 \mathrm{~g}, 21.2 \mathrm{mmol}, 84 \%$ yield) as a colorless oil. $\mathbf{R}_{f}=0.41$ (silica gel, $10 \%$ ethyl acetate in hexanes, UV, $p$-anisaldehyde).

FT-IR (thin film): $v_{\max } 3057,2977,2935,2873,1735,1715,1644,1477,1454,1438,1408,1394,1368$, $1320,1254,1147,1080,1043,1033,1015,949,845,764,676 \mathrm{~cm}^{-1}$.

${ }^{1} \mathbf{H}$ NMR (600 MHz, CDCl 3 ): $\delta 5.90$ (br q, $J=1.4$ Hz, 1 H), 3.39 (br s, $\left.2 \mathrm{H}\right), 2.67-2.63$ (m, $2 \mathrm{H}$ ), 2.50$2.46(\mathrm{~m}, 2 \mathrm{H}), 1.88$ (d, $J=1.4 \mathrm{~Hz}, 3 \mathrm{H}), 1.48$ (s, $9 \mathrm{H}) \mathrm{ppm} .^{*}$

${ }^{13}$ C NMR (151 MHz, CDCl3): $\delta 202.3,166.5,146.1,82.3,75.5,50.7,40.1,32.7,28.1,23.6$ ppm.*

$*$ (ca. $9 \%$ of enol tautomer exists in NMR spectra).

HRMS (ESI, $\boldsymbol{m} / \mathbf{z}$ ): calcd for $\mathrm{C}_{12} \mathrm{H}_{19} \mathrm{IO}_{3} \mathrm{Na}^{+}[\mathrm{M}+\mathrm{Na}]^{+} 361.0271$, found 361.0276, $\Delta 1.4 \mathrm{ppm}$. 
D. Rhoades, ${ }^{*}$ et al.

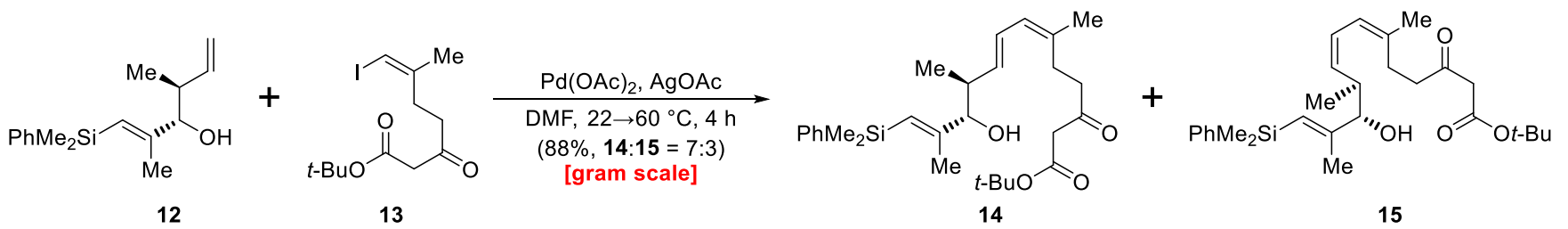

Table S2. Optimization of Mizoroki-Heck coupling of 12 and 13 to afford dienes 14 and 15.

\begin{tabular}{|c|c|c|c|}
\hline entry & conditions $^{\mathrm{a}}$ & yield $^{b}$ & $14: 15^{\mathrm{c}}$ \\
\hline 1 & $\mathrm{Pd}(\mathrm{OAc})_{2}(5 \mathrm{~mol} \%), \mathrm{NaHCO}_{3}$ (2.5 equiv), DMF, $100{ }^{\circ} \mathrm{C}, 3 \mathrm{~h}$ & - & $-{ }^{\mathrm{d}}$ \\
\hline 2 & $\mathrm{Pd}(\mathrm{OAc})_{2}(5 \mathrm{~mol} \%) \mathrm{Ag}_{2} \mathrm{CO}_{3}$ (1.2 equiv), DMF, $22^{\circ} \mathrm{C}, 12 \mathrm{~h}$ & $42 \%$ & $3: 2$ \\
\hline 3 & $\mathrm{Pd}(\mathrm{OAc})_{2}(5 \mathrm{~mol} \%) \mathrm{AgOAc}(1.5$ equiv $), \mathrm{DMF}, 22^{\circ} \mathrm{C}, 12 \mathrm{~h}$ & $54 \%{ }^{\mathrm{e}}$ & $7: 3$ \\
\hline 4 & $\mathrm{Pd}(\mathrm{OAc})_{2}(5 \mathrm{~mol} \%), \mathrm{AgOAc}\left(1.5\right.$ equiv), DMF, $22 \rightarrow 50^{\circ} \mathrm{C}, 4 \mathrm{~h}$ & $60 \% \mathrm{e}^{\mathrm{e}}$ & $7: 3$ \\
\hline 5 & $\mathrm{Pd}(\mathrm{OAc})_{2}(10 \mathrm{~mol} \%), \mathrm{AgOAc}\left(1.5\right.$ equiv), DMF, $22 \rightarrow 60^{\circ} \mathrm{C}, 4 \mathrm{~h}$ & $71 \% \mathrm{e}^{\mathrm{e}}$ & $7: 3$ \\
\hline 6 & $\mathrm{Pd}(\mathrm{OAc})_{2}(10 \mathrm{~mol} \%), \mathrm{AgOAc}\left(1.5\right.$ equiv), NMP, $22 \rightarrow 60^{\circ} \mathrm{C}, 2 \mathrm{~h}$ & $48 \%$ & $2: 1$ \\
\hline 7 & $\mathrm{Pd}(\mathrm{OAc})_{2}(10 \mathrm{~mol} \%), \mathrm{AgOAc}\left(1.5\right.$ equiv), DMSO, $22 \rightarrow 60^{\circ} \mathrm{C}, 1 \mathrm{~h}$ & $8 \%$ & $3: 1^{\mathrm{d}}$ \\
\hline 8 & $\mathrm{Pd}(\mathrm{OAc})_{2}(10 \mathrm{~mol} \%), \mathrm{PPh}_{3}(20 \mathrm{~mol} \%), \mathrm{AgOAc}\left(1.5\right.$ equiv), $\mathrm{DMF}, 70^{\circ} \mathrm{C}, 1 \mathrm{~h}$ & - & $-^{\mathrm{d}}$ \\
\hline 9 & $\mathrm{Pd}(\mathrm{OAc})_{2}(10 \mathrm{~mol} \%), \mathrm{AgOAc}(1.5$ equiv $), \mathrm{DMF}, 22 \rightarrow 80^{\circ} \mathrm{C}, 1 \mathrm{~h}$ & $54 \%$ & $7: 3^{\mathrm{d}}$ \\
\hline 10 & $\mathrm{Pd}(\mathrm{OAc})_{2}(10 \mathrm{~mol} \%), \mathrm{AgOAc}\left(1.5\right.$ equiv), DMF, $22 \rightarrow 100^{\circ} \mathrm{C}, 30 \mathrm{~min}$ & $37 \%$ & $7: 3^{\mathrm{d}}$ \\
\hline 11 & $\mathrm{Pd}(\mathrm{OAc})_{2}(15 \mathrm{~mol} \%), \mathrm{AgOAc}$ (1.5 equiv), DMF, $22 \rightarrow 60^{\circ} \mathrm{C}, 4 \mathrm{~h}$ & $91 \%$ & $7: 3$ \\
\hline $12^{\mathrm{f}}$ & $\mathrm{Pd}(\mathrm{OAc})_{2}(15 \mathrm{~mol} \%), \mathrm{AgOAc}\left(1.5\right.$ equiv), DMF, $22 \rightarrow 60{ }^{\circ} \mathrm{C}, 4 \mathrm{~h}$ & $88 \% \mathrm{~g}$ & $7: 3$ \\
\hline 13 & $\begin{array}{c}\mathrm{Pd}(\mathrm{OAc})_{2}(10 \mathrm{~mol} \%), \mathrm{PPh}_{3}(20 \mathrm{~mol} \%), \mathrm{K}_{2} \mathrm{CO}_{3}(2.5 \text { equiv), } \mathrm{Bu} 4 \mathrm{NBr} \text { (1.0 equiv), } \\
10: 1 \mathrm{DMF} / \mathrm{H}_{2} \mathrm{O}, 50{ }^{\circ} \mathrm{C}, 2.5 \mathrm{~h}\end{array}$ & - & $--^{\mathrm{d}}$ \\
\hline 14 & $\mathrm{Pd}(\mathrm{OAc})_{2}(10 \mathrm{~mol} \%), \operatorname{AgTFA}\left(2.0\right.$ equiv), $\mathrm{K}_{2} \mathrm{CO}_{3}\left(3.0\right.$ equiv), DMF, $60{ }^{\circ} \mathrm{C}, 3 \mathrm{~h}$ & $10 \%$ & $2: 1$ \\
\hline 15 & 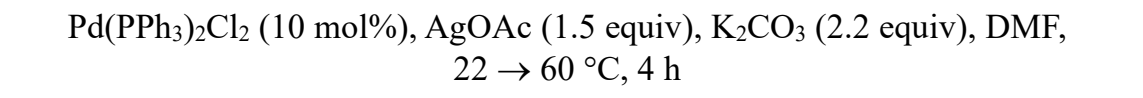 & $<5 \%$ & $4: 1^{\mathrm{d}}$ \\
\hline 16 & $\mathrm{Pd}(\mathrm{OAc})_{2}(10 \mathrm{~mol} \%), \mathrm{Ag}_{3} \mathrm{PO}_{4}$ (1.0 equiv), $\mathrm{K}_{2} \mathrm{CO}_{3}\left(2.0\right.$ equiv), $\mathrm{DMF}, 60^{\circ} \mathrm{C}, 3 \mathrm{~h}$ & $<5 \%$ & $1: 1$ \\
\hline 17 & $\mathrm{Pd}(\mathrm{OAc})_{2}(10 \mathrm{~mol} \%), \mathrm{Ag}_{3} \mathrm{PO}_{4}(1.0$ equiv $), \mathrm{DMF}, 22^{\circ} \mathrm{C}, 12 \mathrm{~h}$ & $32 \%$ & $1.1: 1$ \\
\hline 18 & $\mathrm{Pd}(\mathrm{OAc})_{2}(10 \mathrm{~mol} \%), \mathrm{AgSbF}_{6}$ (2.0 equiv), $\mathrm{K}_{2} \mathrm{CO}_{3}$ (2.0 equiv), $\mathrm{DMF}, 60^{\circ} \mathrm{C}, 3 \mathrm{~h}$ & $10 \%$ & $3: 2$ \\
\hline 19 & $\mathrm{Pd}(\mathrm{OAc})_{2}(10 \mathrm{~mol} \%), \mathrm{AgSbF}_{6}$ (2.0 equiv), DMF, $60^{\circ} \mathrm{C}, 3 \mathrm{~h}$ & $29 \%$ & $3: 2$ \\
\hline 20 & $\operatorname{Pd}(\mathrm{OAc})_{2}(10 \mathrm{~mol} \%)$, BINAP $(20 \mathrm{~mol} \%), \operatorname{AgOAc}(1.5$ equiv $), \mathrm{DMF}, 60^{\circ} \mathrm{C}, 3 \mathrm{~h}$ & - & - \\
\hline
\end{tabular}

${ }^{a}$ All reactions were performed on a $0.10 \mathrm{mmol}$ scale $(26 \mathrm{mg})$ based on 1.0 equiv of $\mathbf{1 2}$, unless otherwise indicated.

${ }^{\text {b} Y i e l d s ~ r e f e r ~ t o ~ a ~ n e a r l y ~ i n s e p a r a b l e ~ m i x t u r e ~ o f ~} \mathbf{1 4}$ and $\mathbf{1 5}$ obtained following purification by flash column chromatography.

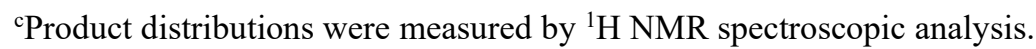

dSignificant decomposition of $\mathbf{1 3}$ was observed.

'Incomplete conversion of starting materials, presumably due to low catalyst loading and deposition of palladium black.

fReaction was performed on gram scale.

gPlus 10\% recovered 12.

tert-Butyl

$(6 Z, 8 E, 10 S, 11 S, 12 E)-13$-[dimethyl(phenyl)silyl]-11-hydroxy-6,10,12-trimethyl-3-

oxotrideca-6,8,12-trienoate (14): To a stirred solution of alcohol 12 (1.02 g, $3.92 \mathrm{mmol}, 1.0$ equiv) and vinyl iodide 13 (1.59 g, $4.70 \mathrm{mmol}, 1.2$ equiv) in dimethylformamide $(56 \mathrm{~mL})$ at $22{ }^{\circ} \mathrm{C}$ was added palladium(II) acetate (132 mg, $0.588 \mathrm{mmol}, 0.15$ equiv), followed by silver(I) acetate $(981 \mathrm{mg}, 5.88$ 


\section{Rhoades, * et al.}

mmol, 1.5 equiv). The reaction mixture was covered with aluminum foil to shield it from ambient light, and stirring was continued for $15 \mathrm{~min}$ at the same temperature. Then the reaction mixture was heated to $60{ }^{\circ} \mathrm{C}$, stirred for $4 \mathrm{~h}$, and then allowed to cool to $22^{\circ} \mathrm{C}$. The reaction mixture was filtered through a layer of celite and rinsed thoroughly with ethyl acetate $(150 \mathrm{~mL})$. The filtrate was washed with brine $(3 \times 20$ $\mathrm{mL}$ ), dried over anhydrous sodium sulfate, and concentrated under reduced pressure. The obtained residue was purified by flash column chromatography (silica gel, $5 \rightarrow 10 \rightarrow 20 \%$ ethyl acetate in hexanes) to afford unreacted olefin (102 mg, $0.392 \mathrm{mmol}$ ) and geometric diene isomers 14 and 15 (1.62 g, $3.45 \mathrm{mmol}, 88 \%$ yield, $98 \%$ brsm, 14:15 = 7:3, 62\% overall yield for 14) as colorless oils. An analytically pure sample of 14 was obtained via repeated preparative thin layer chromatography (silica gel, 20\% diethyl ether in hexanes $\times 3$ runs).

$\mathbf{R}_{f}=0.19$ (silica gel, 20\% diethyl ether in hexanes $\times 3$ runs, UV, $p$-anisaldehyde).

$[\boldsymbol{\alpha}]_{\mathbf{D}}^{22}=-21.7\left(c=0.60, \mathrm{CH}_{2} \mathrm{Cl}_{2}\right)$.

FT-IR (thin film): $v_{\max } 3475,3069,2963,2928,2873,1734,1715,1674,1619,1455,1427,1408,1394$, $1369,1320,1248,1147,1112,1080,1033,1013,965,837,779,731,700,662 \mathrm{~cm}^{-1}$.

${ }^{1} \mathbf{H}$ NMR (600 MHz, CDCl 3$): \delta 7.55-7.51(\mathrm{~m}, 2 \mathrm{H}), 7.36-7.32(\mathrm{~m}, 3 \mathrm{H}), 6.34(\mathrm{dd}, J=15.1,11.0 \mathrm{~Hz}, 1$ H), $5.84(\mathrm{br} \mathrm{d}, J=11.0 \mathrm{~Hz}, 1 \mathrm{H}), 5.61(\mathrm{q}, J=0.7 \mathrm{~Hz}, 1 \mathrm{H}), 5.46(\mathrm{dd}, J=15.1,8.6 \mathrm{~Hz}, 1 \mathrm{H}), 3.73(\mathrm{~d}, J=$ $7.8 \mathrm{~Hz}, 1 \mathrm{H}), 3.35$ (br s, $2 \mathrm{H}), 2.64-2.62(\mathrm{~m}, 2 \mathrm{H}), 2.47-2.44$ (m, $2 \mathrm{H}), 2.34$ (ddq, $J=8.6,7.8,6.8 \mathrm{~Hz}, 1$ H), 1.86 (br s, $1 \mathrm{H}), 1.75$ (br s, $3 \mathrm{H}), 1.69(\mathrm{~d}, J=0.7 \mathrm{~Hz}, 3 \mathrm{H}), 1.47(\mathrm{~s}, 9 \mathrm{H}), 0.96(\mathrm{~d}, J=6.8 \mathrm{~Hz}, 3 \mathrm{H})$, 0.38 (s, $3 \mathrm{H}), 0.37$ (s, $3 \mathrm{H})$ ppm.*

${ }^{13}$ C NMR (151 MHz, CDCl): $\delta$ 203.0, 166.6, 155.8, 139.7, 136.1, 134.1, 133.9, 128.9, 128.1, 127.9, $126.3,124.4,83.1,82.2,50.9,41.6,41.3,28.1,26.2,23.6,17.5,17.1,-0.9,-1.0$ ppm.*

(*ca. $9 \%$ of enol tautomer exists in NMR spectra).

HRMS (ESI, $\boldsymbol{m} / z$ ): calcd for $\mathrm{C}_{28} \mathrm{H}_{42} \mathrm{O}_{4} \mathrm{SiNa}^{+}[\mathrm{M}+\mathrm{Na}]^{+} 493.2745$, found 493.2755, $\Delta 2.0 \mathrm{ppm}$. 


\section{tert-Butyl}

D. Rhoades, * et al.

oxotrideca-6,8,12-trienoate (15): Isolated as an inseparable mixture with $14(\mathbf{1 4 : 1 5}=7: 3)$.

$\boldsymbol{R}_{\mathbf{f}}=0.18$ (silica gel, 20\% diethyl ether in hexanes $\times 3$ runs, UV, $p$-anisaldehyde).

${ }^{1}$ H NMR (600 MHz, CDCl3): $\delta 7.54-7.52$ (m, $\left.2 \mathrm{H}\right), 7.35-7.32(\mathrm{~m}, 3 \mathrm{H}), 6.34$ (br dd, $J=11.3,10.5 \mathrm{~Hz}$ $1 \mathrm{H}), 6.10(\mathrm{br} \mathrm{d}, J=11.3 \mathrm{~Hz}, 1 \mathrm{H}), 5.61$ (br s, $1 \mathrm{H}), 5.21(\mathrm{br} \mathrm{dd}, J=10.5,10.5 \mathrm{~Hz}, 1 \mathrm{H}), 3.73(\mathrm{~d}, J=8.1$ Hz, $1 \mathrm{H}), 3.35$ (br s, $2 \mathrm{H}), 2.77$ (ddq, $J=10.5,8.1,6.7 \mathrm{~Hz}, 1 \mathrm{H}), 2.63-2.61$ (m, $2 \mathrm{H}), 2.47-2.43$ (m, $2 \mathrm{H})$, 1.81 (br s, $1 \mathrm{H}), 1.79$ (br s, $3 \mathrm{H}), 1.72$ (br s, $3 \mathrm{H}), 1.47$ (s, $9 \mathrm{H}), 0.90$ (d, J = 6.7 Hz, $3 \mathrm{H}), 0.38$ (s, $3 \mathrm{H})$, 0.37 (s, $3 \mathrm{H}) \mathrm{ppm} . *$

${ }^{13}$ C NMR (151 MHz, CDCl3): $\delta$ 202.8, 166.5, 155.7, 139.6, 138.5, 132.3, 128.9, 128.1, 127.9, 126.4, $124.9,121.5,83.9,82.2,50.8,41.4,36.5,28.5,26.1,24.2,17.8,17.0,-0.96,-1.03$ ppm.* (*ca. $9 \%$ of enol tautomer exists in NMR spectrum).

HRMS (ESI, $\boldsymbol{m} / \boldsymbol{z}$ ): calcd for $\mathrm{C}_{28} \mathrm{H}_{42} \mathrm{O}_{4} \mathrm{SiNa}^{+}[\mathrm{M}+\mathrm{Na}]^{+} 493.2745$, found 493.2752, $\Delta 1.4$ ppm. 
D. Rhoades, * et al.

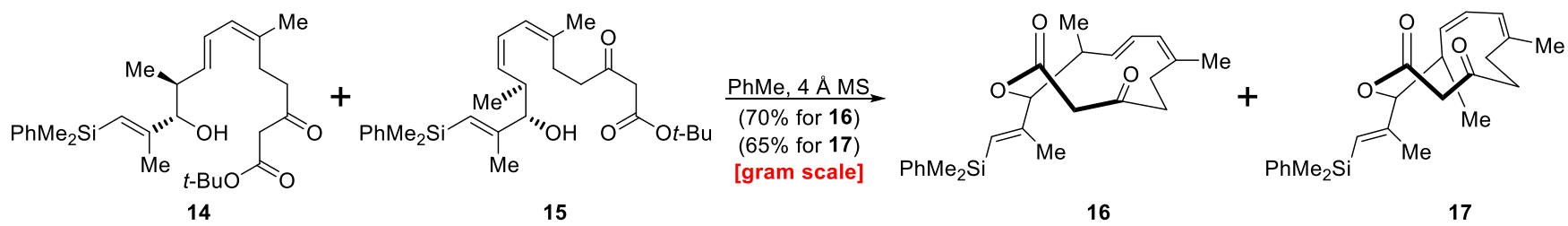

Table S3. Optimization of the macrolactonization of $\mathbf{1 4}$ and $\mathbf{1 5}$ to afford macrolides $\mathbf{1 6}$ and $\mathbf{1 7 .}$

\begin{tabular}{ccc}
\hline entry & conditions $^{\mathrm{a}}$ & yield $^{\mathrm{b}}$ \\
\hline 1 & $\mathrm{PhH}(0.05 \mathrm{M}), 80^{\circ} \mathrm{C}, 12 \mathrm{~h}$ & $18 \%^{\mathrm{c}}$ \\
2 & $\mathrm{PhH}(0.05 \mathrm{M}), 100^{\circ} \mathrm{C}, 4 \mathrm{~h}$ & $24 \%^{\mathrm{c}}$ \\
3 & $\mathrm{PhH}\left(4 \times 10^{-3} \mathrm{M}\right), 100^{\circ} \mathrm{C}, 6 \mathrm{~h}$ & $28 \%^{\mathrm{c}}$ \\
4 & $\mathrm{PhCl}(0.05 \mathrm{M}), 130^{\circ} \mathrm{C}, 6 \mathrm{~h}$ & $21 \%{ }^{\mathrm{c}}$ \\
5 & $\mathrm{PhMe}(0.1 \mathrm{M}), 110^{\circ} \mathrm{C}, 3 \mathrm{~h}$ & $30 \%^{\mathrm{c}}$ \\
6 & $\mathrm{PhMe}(0.05 \mathrm{M}), 110^{\circ} \mathrm{C}, 6 \mathrm{~h}$ & $34 \%{ }^{\mathrm{c}, \mathrm{d}}$ \\
7 & $\mathrm{PhMe}(0.05 \mathrm{M}), 125^{\circ} \mathrm{C}, 4 \mathrm{~h}$ & $38 \%{ }^{\mathrm{c}, \mathrm{d}}$ \\
8 & $\mathrm{PhMe}\left(4 \times 10^{-3} \mathrm{M}\right), 125^{\circ} \mathrm{C}, 4 \mathrm{~h}$ & $47 \%{ }^{\mathrm{c}, \mathrm{d}}$ \\
9 & $\mathrm{PhMe}\left(4 \times 10^{-3} \mathrm{M}\right), 4 \AA \mathrm{MS}, 125{ }^{\circ} \mathrm{C}, 3 \mathrm{~h}$ & $54 \%{ }^{\mathrm{c}, \mathrm{d}}$
\end{tabular}

$10^{\text {e, f }} \quad \operatorname{PhMe}\left(4 \times 10^{-3} \mathrm{M}\right), 4 \AA \mathrm{MS}, 125^{\circ} \mathrm{C}, 15 \mathrm{~min} \quad 70 \%$

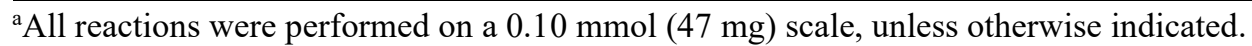

${ }^{b}$ Yields refer to amount of $\mathbf{1 6}$ obtained following purification by flash column chromatography.

${ }^{\mathrm{c} S}$ Significant and variable levels of decarboxylation of the corresponding $\beta$-ketoester were observed.

${ }^{d}$ Yields decreased substantially upon scale up and were generally irreproducible.

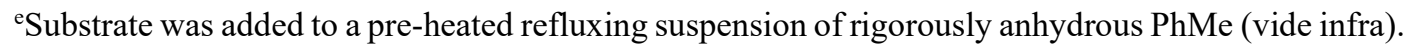

fReaction performed on gram scale.

(7Z,9E,11S,12S)-12-\{(E)-1-[Dimethyl(phenyl)silyl]prop-1-en-2-yl\}-7,11-dimethyloxacyclododeca-

7,9-diene-2,4-dione (16): To a vigorously stirred suspension of freshly activated $4 \AA$ molecular sieves $(18 \mathrm{~g})$ in refluxing toluene $(770 \mathrm{~mL})$ at $125^{\circ} \mathrm{C}$ was added a solution of $\beta$-ketoesters 14 and $\mathbf{1 5}(\mathbf{1 4}: 15=$ 7:3, $1.45 \mathrm{~g}, 3.08 \mathrm{mmol}, 1.0$ equiv) in toluene $(20 \mathrm{~mL})$ in a single portion via syringe, and the original flask was quickly rinsed with additional toluene $(3 \times 5 \mathrm{~mL})$. After stirring for $15 \mathrm{~min}$ at the same temperature, the reaction mixture was allowed to cool to $22{ }^{\circ} \mathrm{C}$. Then the reaction mixture was filtered through a layer of celite, rinsed thoroughly with ethyl acetate $(250 \mathrm{~mL})$, and concentrated under reduced pressure. The obtained residue was purified by flash column chromatography (silica gel, $2 \rightarrow 5 \rightarrow 10 \%$ ethyl acetate in hexanes) to afford $\beta$-ketolactones 16 (599 mg, $1.51 \mathrm{mmol}, 70 \%$ yield based on 14) and 17 (238 mg, 0.600 mmol, $65 \%$ yield based on $\mathbf{1 5}$ ) as colorless oils. 


\section{Rhoades, * et al.}

$\mathbf{R}_{f}=0.22$ (silica gel, 20\% diethyl ether in hexanes, UV, $p$-anisaldehyde).

$[\boldsymbol{\alpha}]_{\mathbf{D}}^{22}=+63.6\left(c=0.50, \mathrm{CH}_{2} \mathrm{Cl}_{2}\right)$.

FT-IR (thin film): $v_{\max } 3069,2963,2925,2874,2853,1741,1716,1617,1453,1427,1407,1378,1329$, $1249,1162,1113,1059,1020,984,910,889,836,780,731,701,662 \mathrm{~cm}^{-1}$.

${ }^{1}$ H NMR (600 MHz, CDCl $): \delta 7.52-7.49$ (m, 2 H), 7.36-7.32 (m, 3 H), $5.95(\mathrm{dd}, J=15.4,9.7$ Hz, 1 H), $5.79(\mathrm{dq}, J=9.7,1.3 \mathrm{~Hz}, 1 \mathrm{H}), 5.71(\mathrm{br} \mathrm{q}, J=0.7 \mathrm{~Hz}, 1 \mathrm{H}), 5.16(\mathrm{dd}, J=15.4,8.4 \mathrm{~Hz}, 1 \mathrm{H}), 4.86(\mathrm{~d}, J=$ $10.2 \mathrm{~Hz}, 1 \mathrm{H}), 3.54(\mathrm{dd}, J=12.9,0.6 \mathrm{~Hz}, 1 \mathrm{H}), 3.30(\mathrm{~d}, J=12.9 \mathrm{~Hz}, 1 \mathrm{H}), 2.94$ (dddd, $J=14.1,6.7,5.1$, $1.3 \mathrm{~Hz}, 1 \mathrm{H}), 2.70$ (ddd, $J=16.1,8.9,5.1 \mathrm{~Hz}, 1 \mathrm{H}), 2.58$ (dddd, $J=16.1,6.7,5.0,0.6 \mathrm{~Hz}, 1 \mathrm{H}), 2.33$ (ddq, $J=10.2,8.4,6.9 \mathrm{~Hz}, 1 \mathrm{H}), 2.01(\mathrm{ddd}, J=14.1,8.9,5.0 \mathrm{~Hz}, 1 \mathrm{H}), 1.79(\mathrm{br} \mathrm{d}, J=1.3 \mathrm{~Hz}, 3 \mathrm{H}), 1.69$ (d, $J$ $=0.7 \mathrm{~Hz}, 3 \mathrm{H}), 0.92(\mathrm{~d}, J=6.9 \mathrm{~Hz}, 3 \mathrm{H}), 0.38(\mathrm{~s}, 3 \mathrm{H}), 0.37(\mathrm{~s}, 3 \mathrm{H}) \mathrm{ppm}$.

${ }^{13}$ C NMR (151 MHz, CDCl3): $\delta$ 201.2, 164.5, 150.8, 139.2, 138.8, 133.8, 132.9, 129.8, 129.1, 128.7, $128.0,124.8,86.4,50.2,43.1,39.2,26.4,24.8,16.9,16.3,-1.1,-1.2 \mathrm{ppm}$.

HRMS (ESI, $\boldsymbol{m} / \boldsymbol{z}$ ): calcd for $\mathrm{C}_{24} \mathrm{H}_{32} \mathrm{O}_{3} \mathrm{SiNa}^{+}[\mathrm{M}+\mathrm{Na}]^{+}$419.2013, found 419.2024, $\Delta 2.6 \mathrm{ppm}$.

\section{(7Z,9Z,11S,12S)-12-\{(E)-1-[Dimethyl(phenyl)silyl]prop-1-en-2-yl\}-7,11-dimethyloxacyclododeca-}

7,9-diene-2,4-dione (17): $\mathbf{R}_{\boldsymbol{f}}=0.19$ (silica gel, 20\% diethyl ether in hexanes, UV, $p$-anisaldehyde).

$[\alpha]_{\mathbf{D}}^{22}=-216\left(c=0.50, \mathrm{CH}_{2} \mathrm{Cl}_{2}\right)$.

FT-IR (thin film): $v_{\max } 2965,2924,2867,2845,1752,1714,1618,1454,1428,1406,1377,1332,1295$, $1249,1177,1155,1113,1087,1055,1013,931,835,814,779,731,701 \mathrm{~cm}^{-1}$.

${ }^{1}$ H NMR (600 MHz, CDCl3): $\delta 7.52-7.49$ (m, 2 H), 7.37-7.32 (m, 3 H), 6.08 (ddd, J = 10.8, 8.9, 1.1 Hz, $1 \mathrm{H}), 5.98(\mathrm{dq}, J=8.9,1.4 \mathrm{~Hz}, 1 \mathrm{H}), 5.71(\mathrm{br} \mathrm{q}, J=0.7 \mathrm{~Hz}, 1 \mathrm{H}), 5.11(\mathrm{ddd}, J=10.8,10.8,1.0 \mathrm{~Hz}, 1 \mathrm{H})$, $4.90(\mathrm{~d}, J=10.4 \mathrm{~Hz}, 1 \mathrm{H}), 3.33(\mathrm{dd}, J=14.8,0.6 \mathrm{~Hz}, 1 \mathrm{H}), 3.23(\mathrm{~d}, J=14.8 \mathrm{~Hz}, 1 \mathrm{H}), 3.00(\mathrm{dddd}, J=$ 14.7, 9.5, 4.3 Hz, 0.6 Hz, $1 \mathrm{H}), 2.77$ (ddq, $J=10.8,10.4,6.7 \mathrm{~Hz}, 1 \mathrm{H}), 2.76$ (ddd, $J=13.7,7.6,4.3 \mathrm{~Hz}, 1$ H), $2.48(\mathrm{ddd}, J=14.7,7.6,4.1 \mathrm{~Hz}, 1 \mathrm{H}), 2.12(\mathrm{ddd}, J=13.7,9.5,4.1 \mathrm{~Hz}, 1 \mathrm{H}), 1.89$ (br d, $J=1.4 \mathrm{~Hz}, 3$ H), $1.67(\mathrm{~d}, J=0.7 \mathrm{~Hz}, 3 \mathrm{H}), 0.88(\mathrm{~d}, J=6.7 \mathrm{~Hz}, 3 \mathrm{H}), 0.38(\mathrm{~s}, 3 \mathrm{H}), 0.37$ (s, $3 \mathrm{H}) \mathrm{ppm}$. 


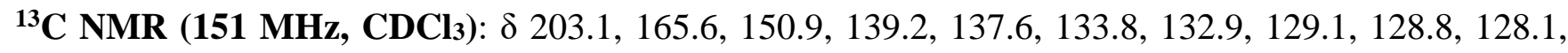
128.0, 122.5, 86.1, 51.2, 40.7, 34.6, 28.4, 25.6, 16.9, 16.5, -1.0, -1.2 ppm.

HRMS (ESI, $\boldsymbol{m} / \mathbf{z}$ ): calcd for $\mathrm{C}_{24} \mathrm{H}_{32} \mathrm{O}_{3} \mathrm{SiNa}^{+}[\mathrm{M}+\mathrm{Na}]^{+} 419.2013$, found 419.2008, $\Delta 1.2 \mathrm{ppm}$.

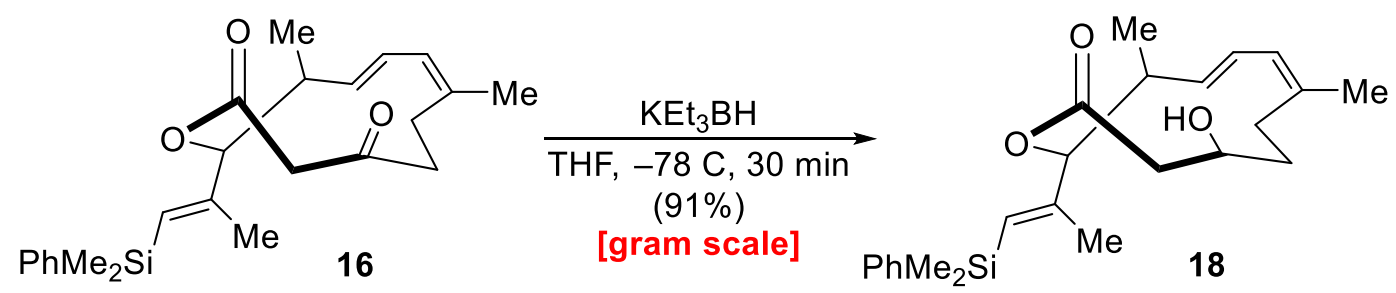

Table S4. Optimization of the reduction of $\beta$-ketolactone $\mathbf{1 6}$ to afford $\beta$-hydroxylactone $\mathbf{1 8 .}$

\begin{tabular}{|c|c|c|}
\hline entry & conditions $^{\mathrm{a}}$ & yield $^{b}$ \\
\hline 1 & $\mathrm{NaBH}_{4}$ (3.0 equiv), EtOH, $0^{\circ} \mathrm{C}, 1 \mathrm{~h}$ & $58 \%{ }^{\mathrm{c}}$ \\
\hline 2 & $\mathrm{NaBH}_{4}$ (3.0 equiv), $1: 1 \mathrm{THF} / i-\mathrm{PrOH}, 0^{\circ} \mathrm{C}, 1 \mathrm{~h}$ & $47 \%{ }^{c}$ \\
\hline $3^{c}$ & $\mathrm{NaBH}_{4}\left(3.0\right.$ equiv), EtOH, $0^{\circ} \mathrm{C}, 1 \mathrm{~h}$ & $28 \%$ c,d \\
\hline 4 & K-selectride ( 1.5 equiv), THF, $-78^{\circ} \mathrm{C}, 2 \mathrm{~h}$ & $49 \%$ \\
\hline 5 & K-selectride ( 7.0 equiv), THF, $-78 \rightarrow-20^{\circ} \mathrm{C}, 3 \mathrm{~h}$ & $30 \% \mathrm{e}^{\mathrm{e}}$ \\
\hline 6 & L-selectride (5.0 equiv), THF, $-78 \rightarrow 0^{\circ} \mathrm{C}, 3 \mathrm{~h}$ & $24 \% \mathrm{e}^{\mathrm{e}}$ \\
\hline 7 & DIBAL ( 1.5 equiv), $\mathrm{PhMe},-78^{\circ} \mathrm{C}, 1 \mathrm{~h}$ & $41 \%$ \\
\hline 8 & $\mathrm{LiAlH}(\mathrm{O} t-\mathrm{Bu})_{3}(1.5$ equiv $), \mathrm{THF}, 0^{\circ} \mathrm{C}, 3 \mathrm{~h}$ & $18 \%$ \\
\hline 9 & $\mathrm{KEt}_{3} \mathrm{BH}$ (1.0 equiv), THF, $-78^{\circ} \mathrm{C}, 30 \mathrm{~min}$ & $85 \%$ \\
\hline $10^{\mathrm{f}}$ & $\mathrm{KEt}_{3} \mathrm{BH}$ (1.0 equiv), THF, $-78^{\circ} \mathrm{C}, 30 \mathrm{~min}$ & $91 \%$ \\
\hline
\end{tabular}

${ }^{a}$ All reactions were performed on a $0.1 \mathrm{mmol}(40 \mathrm{mg})$ scale, unless otherwise indicated.

${ }^{\text {b}}$ Yields refer to amount of $\mathbf{1 8}$ obtained following purification by flash column chromatography.

'Incomplete conversion of $\mathbf{1 6}$ and inconsistent diastereoselectivity were observed.

${ }^{\mathrm{d}}$ Reaction performed on $0.30 \mathrm{mmol}(120 \mathrm{mg})$ scale.

eIncomplete conversion of 16, even with excess reagent and elevated reaction temperatures.

${ }^{\mathrm{f}}$ Reaction performed on gram scale.

\section{$(4 R, 7 Z, 9 E, 11 S, 12 S)-12-\{(E)-1-[D i m e t h y l(p h e n y l) s i l y l] p r o p-1-e n-2-y l\}-4-h y d r o x y-7,11-$}

dimethyloxacyclododeca-7,9-dien-2-one (18): To a stirred solution of $\beta$-ketolactone 16 (1.19 g, 3.00 mmol, 1.0 equiv) in tetrahydrofuran $(30 \mathrm{~mL})$ at $-78^{\circ} \mathrm{C}$ was carefully added a solution of potassium triethylborohydride (1.0 M tetrahydrofuran, $3.0 \mathrm{~mL}, 3.0 \mathrm{mmol}, 1.0$ equiv) dropwise. After stirring for 30 min at the same temperature, the reaction mixture was quenched by the addition of methanol $(120 \mu \mathrm{L}, 3.0$ mmol, 1.0 equiv) and a saturated aqueous solution of ammonium chloride (40 mL), and allowed to warm 


\section{Rhoades, * et al.}

to $22^{\circ} \mathrm{C}$. The two phases were separated, and the aqueous layer was extracted with ethyl acetate $(3 \times 10$ $\mathrm{mL}$ ). The combined organic phases were dried over anhydrous sodium sulfate and concentrated under reduced pressure. The obtained residue was purified by flash column chromatography (silica gel, $5 \rightarrow 15 \rightarrow 30 \%$ ethyl acetate in hexanes) to afford alcohol $\mathbf{1 8}(1.09 \mathrm{~g}, 2.73 \mathrm{mmol}, 91 \%$ yield) as a colorless oil.

$\mathbf{R}_{f}=0.32$ (silica gel, 20\% ethyl acetate in hexanes, UV, $p$-anisaldehyde). $[\boldsymbol{\alpha}]_{\mathbf{D}}^{22}=+45.6\left(c=0.50, \mathrm{CH}_{2} \mathrm{Cl}_{2}\right)$.

FT-IR (thin film): $v_{\max } 3447,3069,3048,2959,2927,2872,2855,1731,1713,1617,1455,1428,1367$, $1248,1172,1113,1086,1061,1025,969,945,836,779,731,700 \mathrm{~cm}^{-1}$.

${ }^{1} \mathbf{H}$ NMR (600 MHz, CDCl3): $\delta$ 7.53-7.49 (m, $\left.2 \mathrm{H}\right), 7.37-7.32(\mathrm{~m}, 3 \mathrm{H}), 6.07(\mathrm{dd}, J=15.8,6.1 \mathrm{~Hz}, 1 \mathrm{H})$, $5.74($ br q, $J=0.7 \mathrm{~Hz}, 1 \mathrm{H}), 5.71(\mathrm{dq}, J=6.1,1.5 \mathrm{~Hz}, 1 \mathrm{H}), 5.15(\mathrm{ddd}, J=15.8,8.3,1.5 \mathrm{~Hz}, 1 \mathrm{H}), 4.98$ (d, $J=10.4 \mathrm{~Hz}, 1 \mathrm{H}), 3.83$ (ddddd, $J=11.6,10.7,4.3,3.7,3.1 \mathrm{~Hz}, 1 \mathrm{H}), 3.34(\mathrm{~d}, J=10.7 \mathrm{~Hz}, 1 \mathrm{H}), 2.76$ $(\mathrm{dd}, J=15.1,3.1 \mathrm{~Hz}, 1 \mathrm{H}), 2.70(\mathrm{dd}, J=15.1,4.3 \mathrm{~Hz}, 1 \mathrm{H}), 2.46(\mathrm{ddq}, J=10.4,8.3,6.9 \mathrm{~Hz}, 1 \mathrm{H}), 2.30$ (ddd, $J=13.0,12.6,2.5 \mathrm{~Hz}, 1 \mathrm{H}), 2.04$ (dddd, $J=13.6,13.0,6.6,3.7 \mathrm{~Hz}, 1 \mathrm{H}), 1.75$ (br d, $J=1.5 \mathrm{~Hz}, 3$ H), $1.69(\mathrm{ddd}, J=12.6,12.1,6.6 \mathrm{~Hz}, 1 \mathrm{H}), 1.67(\mathrm{~d}, J=0.7 \mathrm{~Hz}, 3 \mathrm{H}), 1.26$ (dddd, $J=13.6,12.1,11.6,2.5$ $\mathrm{Hz}, 1 \mathrm{H}), 0.93(\mathrm{~d}, J=6.9 \mathrm{~Hz}, 3 \mathrm{H}), 0.39(\mathrm{~s}, 3 \mathrm{H}), 0.37$ (s, $3 \mathrm{H}) \mathrm{ppm}$.

${ }^{13}$ C NMR (151 MHz, CDCl3): $\delta$ 172.2, 150.8, 140.8, 139.2, 133.8, 133.6, 130.0, 129.1, 128.9, 128.0, $122.9,85.0,69.5,39.2,38.8,36.6,29.6,23.4,17.0,16.9,-1.0,-1.2 \mathrm{ppm}$.

HRMS (ESI, $\boldsymbol{m} / z$ ): calcd for $\mathrm{C}_{24} \mathrm{H}_{34} \mathrm{O}_{3} \mathrm{SiNa}^{+}[\mathrm{M}+\mathrm{Na}]^{+} 421.2169$, found 421.2167, $\Delta 0.5 \mathrm{ppm}$. 
D. Rhoades, * et al.

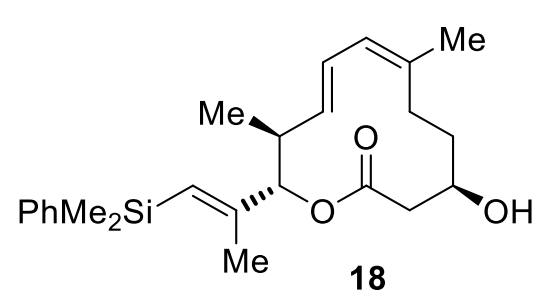

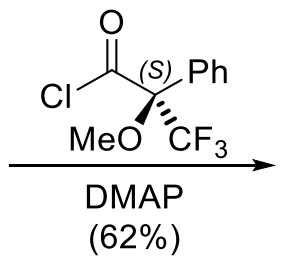

$(62 \%)$

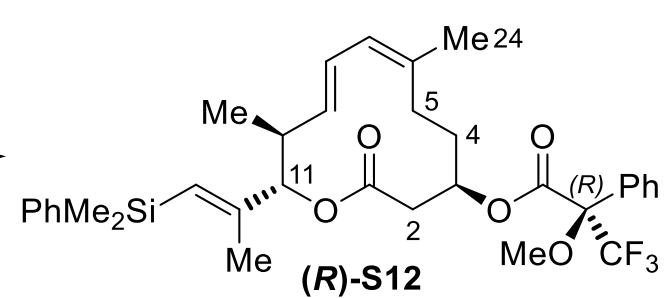

$(4 R, 7 Z, 9 E, 11 S, 12 S)-12-\{(E)-1-[D i m e t h y l(p h e n y l) s i l y l] p r o p-1-e n-2-y l\}-7,11-d i m e t h y l-2-$

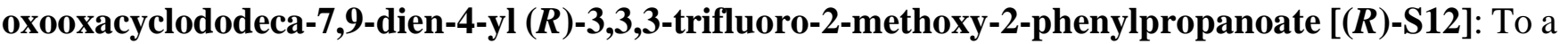
stirred solution of alcohol $18\left(5.2 \mathrm{mg}, 13 \mu \mathrm{mol}, 1.0\right.$ equiv) in dichloromethane $(1.0 \mathrm{~mL})$ at $22{ }^{\circ} \mathrm{C}$ was added $(S)-(+)-\alpha$-methoxy- $\alpha$-(trifluoromethyl)phenylacetyl chloride $(7.0 \mu \mathrm{L}, 39 \mu \mathrm{mol}, 3.0$ equiv), followed by 4-dimethylaminopyridine ( $6.4 \mathrm{mg}, 52 \mu \mathrm{mol}, 4.0$ equiv). After stirring for $4 \mathrm{~h}$, the reaction mixture was concentrated under reduced pressure and purified directly by preparative thin layer chromatography $(5 \%$ ethyl acetate in hexanes) to afford Mosher ester $(\boldsymbol{R})-\mathbf{S 1 2}(5.0 \mathrm{mg}, 8.1 \mu \mathrm{mol}, 62 \%$ yield) as a colorless oil. $\mathbf{R}_{f}=0.20$ (silica gel, 5\% ethyl acetate in hexanes, UV, $p$-anisaldehyde).

$[\boldsymbol{\alpha}]_{\mathbf{D}}^{22}=+88.0\left(c=0.50, \mathrm{CH}_{2} \mathrm{Cl}_{2}\right)$.

FT-IR (thin film): $v_{\max } 3069,3045,2960,2928,2875,2854,1741,1618,1487,1452,1428,1378,1324$, $1248,1169,1113,1082,1066,1023,997,970,946,922,905,893,836,816,778,765,731,718$, $700 \mathrm{~cm}^{-1}$.

${ }^{1}$ H NMR (600 MHz, CDCl3): $\delta$ 7.54-7.50 (m, 4 H), 7.43-7.38 (m, 3 H), 7.37-7.33 (m, 3 H), 6.07 (dd, J $=15.7,8.1 \mathrm{~Hz}, 1 \mathrm{H}), 5.82(\mathrm{dq}, J=8.1,0.9 \mathrm{~Hz}, 1 \mathrm{H}), 5.74($ br s, $1 \mathrm{H}), 5.44(\mathrm{dddd}, J=9.7,8.5,5.9,4.2 \mathrm{~Hz}$, $1 \mathrm{H}), 5.19(\mathrm{dd}, J=15.7,8.6 \mathrm{~Hz}, 1 \mathrm{H}), 4.87(\mathrm{~d}, J=10.3 \mathrm{~Hz}, 1 \mathrm{H}), 3.57(\mathrm{~s}, 3 \mathrm{H}), 2.86(\mathrm{dd}, J=14.8,4.2 \mathrm{~Hz}$, $1 \mathrm{H}), 2.48(\mathrm{ddq}, J=10.3,8.6,6.8 \mathrm{~Hz}, 1 \mathrm{H}), 2.44(\mathrm{dd}, J=14.8,8.5 \mathrm{~Hz}, 1 \mathrm{H}), 2.35$ (ddd, $J=11.8,7.8,6.6$ Hz, 1 H), 2.05-1.97 (m, 2 H), 1.76 (br s, 3 H), 1.69 (d, $J=0.9$ Hz, 3 H), 1.67 (dddd, $J=13.8,10.9,7.8$, $5.9 \mathrm{~Hz}, 1 \mathrm{H}), 0.94$ (d, $J=6.8 \mathrm{~Hz}, 3 \mathrm{H}), 0.38$ (s, $3 \mathrm{H}), 0.37$ (s, $3 \mathrm{H}) \mathrm{ppm}$.

${ }^{13}$ C NMR (151 MHz, CDCl3): $\delta$ 168.0, 166.4, 150.9, 140.3, 139.2, 133.8, 132.9, 132.2, 130.4, 129.8, 129.1, 128.7, 128.6, 128.0, 127.4, 124.1, $123.4(\mathrm{q}, J=288.3 \mathrm{~Hz}), 85.3,84.7$ (q, $J=27.4 \mathrm{~Hz}), 75.4,55.7$, $39.9,39.3,33.5,30.2,24.4,16.9,16.8,-1.0,-1.2 \mathrm{ppm}$. 


\section{Rhoades, * et al.}

${ }^{19}$ F NMR (565 MHz, CDCl3): $\delta-70.7$ ppm.

HRMS (ESI, $\boldsymbol{m} / z$ ): calcd for $\mathrm{C}_{34} \mathrm{H}_{41} \mathrm{~F}_{3} \mathrm{O}_{5} \mathrm{SiNa}^{+}[\mathrm{M}+\mathrm{Na}]^{+}$637.2568, found 637.2567, $\Delta 0.2 \mathrm{ppm}$.
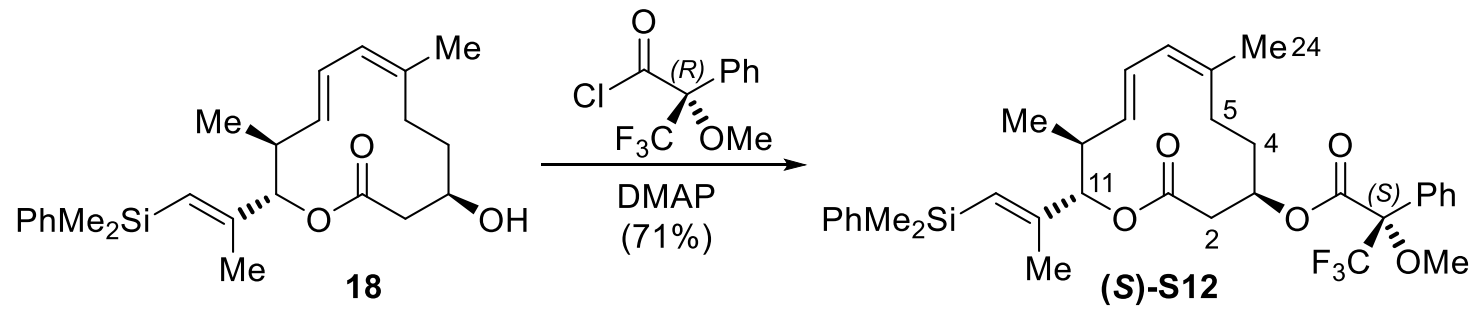

$(4 R, 7 Z, 9 E, 11 S, 12 S)-12-\{(E)-1-[D i m e t h y l(p h e n y l)$ silyl]prop-1-en-2-yl\}-7,11-dimethyl-2-

oxooxacyclododeca-7,9-dien-4-yl $(S)$-3,3,3-trifluoro-2-methoxy-2-phenylpropanoate [(S)-S12]:To a stirred solution of alcohol $18\left(5.6 \mathrm{mg}, 14 \mu \mathrm{mol}, 1.0\right.$ equiv) in dichloromethane $(1.0 \mathrm{~mL})$ at $22{ }^{\circ} \mathrm{C}$ was added $(R)-(-)$ - $\alpha$-methoxy- $\alpha$-(trifluoromethyl)phenylacetyl chloride (8.0 $\mu \mathrm{L}, 42 \mu$ mol, 3.0 equiv), followed by 4-dimethylaminopyridine $(6.8 \mathrm{mg}, 56 \mu \mathrm{mol}, 4.0$ equiv). After stirring for $4 \mathrm{~h}$, the reaction mixture was concentrated under reduced pressure and purified directly by preparative thin layer chromatography (5\% ethyl acetate in hexanes) to afford Mosher ester $(S)-S 12$ (6.4 mg, $0.010 \mathrm{mmol}, 71 \%$ yield) as a colorless oil.

$\mathbf{R}_{f}=0.20$ (silica gel, 5\% ethyl acetate in hexanes, UV, $p$-anisaldehyde).

$[\boldsymbol{\alpha}]_{\mathbf{D}}^{22}=+67.8\left(c=0.60, \mathrm{CH}_{2} \mathrm{Cl}_{2}\right)$.

FT-IR (thin film): $v_{\max } 3069,3045,2961,2928,2874,2853,1741,1618,1488,1452,1428,1378,1322$, $1248,1238,1169,1113,1082,1065,1022,997,970,946,917,903,835,815,780,765,731,718$, $699 \mathrm{~cm}^{-1}$.

${ }^{1} \mathbf{H}$ NMR (600 MHz, CDCl 3$): \delta 7.55-7.49(\mathrm{~m}, 4 \mathrm{H}), 7.43-7.38(\mathrm{~m}, 3 \mathrm{H}), 7.37-7.33$ (m, $\left.3 \mathrm{H}\right), 6.07$ (dd, J $=15.7,7.9 \mathrm{~Hz}, 1 \mathrm{H}), 5.81(\mathrm{dq}, J=7.9,1.1 \mathrm{~Hz}, 1 \mathrm{H}), 5.75(\mathrm{br} \mathrm{s}, 1 \mathrm{H}), 5.44(\mathrm{dddd}, J=8.2,5.9,5.8,4.3,1$ H), $5.18(\mathrm{dd}, J=15.7,8.5 \mathrm{~Hz}, 1 \mathrm{H}), 4.89(\mathrm{~d}, J=10.3 \mathrm{~Hz}, 1 \mathrm{H}), 3.56(\mathrm{~s}, 3 \mathrm{H}), 2.90(\mathrm{dd}, J=14.9,4.3 \mathrm{~Hz}$, $1 \mathrm{H}), 2.59$ (dd, $J=14.9,8.2 \mathrm{~Hz}, 1 \mathrm{H}), 2.48(\mathrm{ddq}, J=10.3,8.5,6.9 \mathrm{~Hz}, 1 \mathrm{H}), 2.30$ (ddd, $J=11.6,7.9,6.7$ 


\section{Rhoades, * et al.}

Hz, 1 H), 1.93-1.85 (m, 2 H), 1.72 (br s, 3 H), 1.68 (d, J = 0.7 Hz, 3 H), 1.62 (dddd, $J=13.8,10.9$, 7.8,

$5.9 \mathrm{~Hz}, 1 \mathrm{H}), 0.94(\mathrm{~d}, J=6.9 \mathrm{~Hz}, 3 \mathrm{H}), 0.38(\mathrm{~s}, 3 \mathrm{H}), 0.37$ (s, $3 \mathrm{H}) \mathrm{ppm}$.

${ }^{13}$ C NMR (151 MHz, CDCl 3$): \delta 168.1,166.3,150.9,140.2,139.2,133.8,132.9,132.3,130.4,129.8$, 129.1, 128.7, 128.6, 128.0, 127.4, 124.0, $123.4(\mathrm{q}, J=288.7 \mathrm{~Hz}), 85.4,84.7(\mathrm{q}, J=27.6 \mathrm{~Hz}), 75.1,55.6$, $40.1,39.4,33.4,29.9,24.3,17.0,16.8,-1.04,-1.14$ ppm.

${ }^{19}$ F NMR (565 MHz, CDCl3): $\delta-70.6 \mathrm{ppm}$.

HRMS (ESI, $m / z$ ): calcd for $\mathrm{C}_{34} \mathrm{H}_{41} \mathrm{~F}_{3} \mathrm{O}_{5} \mathrm{SiNa}^{+}[\mathrm{M}+\mathrm{Na}]^{+}$637.2568, found 637.2564, $\Delta$ 0.6 ppm.

Table S5. Mosher ester analysis of MTPA esters derived from alcohol $\mathbf{1 8 .}$

\begin{tabular}{|c|c|c|c|}
\hline Proton & $\begin{array}{c}\text { Chemical shift of }(S)- \\
\text { MTPA derivative }\end{array}$ & $\begin{array}{c}\text { Chemical shift of }(R)- \\
\text { MTPA derivative }\end{array}$ & $\begin{array}{c}\Delta \delta:(S) \text {-MTPA }- \\
(R)-\text { MTPA }\end{array}$ \\
\hline $\mathbf{2 4}$ & 1.72 & 1.76 & -0.04 \\
\hline $\mathbf{5 a}$ & 2.31 & 2.35 & -0.04 \\
\hline $\mathbf{5 b}$ & 1.91 & 2.01 & -0.10 \\
\hline $\mathbf{4 a}$ & 1.90 & 2.00 & -0.10 \\
\hline $\mathbf{4 b}$ & 1.62 & 1.67 & -0.05 \\
\hline $\mathbf{2 a}$ & 2.90 & 2.86 & +0.04 \\
\hline $\mathbf{2 b}$ & 2.59 & 2.44 & +0.15 \\
\hline $\mathbf{1 1}$ & 4.89 & 4.87 & +0.02 \\
\hline
\end{tabular}

On the basis of the Mosher ester analysis, ${ }^{21}$ the absolute configuration at C3 (pladienolide numbering) is unambiguously assigned as $(R)$.

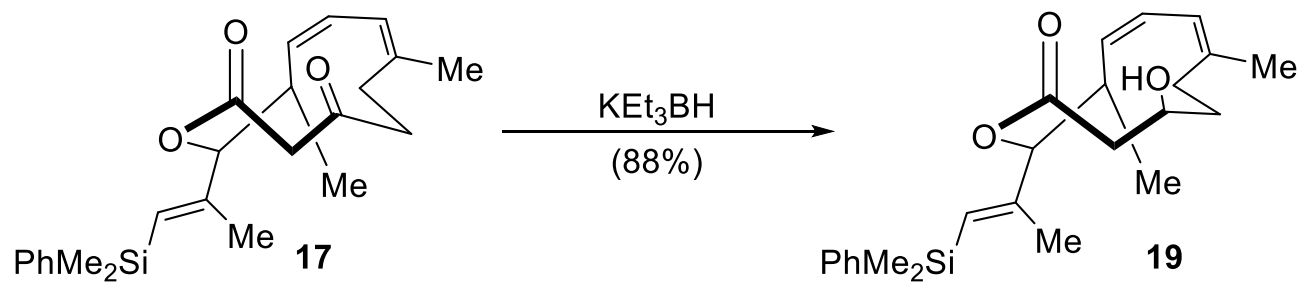

\section{$(4 S, 7 Z, 9 Z, 11 S, 12 S)-12-\{(E)-1-[D i m e t h y l(p h e n y l) s i l y l] p r o p-1-e n-2-y l\}-4-h y d r o x y-7,11-$}

dimethyloxacyclododeca-7,9-dien-2-one (19): To a stirred solution of $\beta$-ketolactone 17 (149 mg, 0.375 mmol, 1.0 equiv) in tetrahydrofuran $(3.8 \mathrm{~mL})$ at $-78{ }^{\circ} \mathrm{C}$ was carefully added a solution of potassium triethylborohydride (1.0 M tetrahydrofuran, $0.38 \mathrm{~mL}, 0.38 \mathrm{mmol}, 1.0$ equiv) dropwise. After stirring for 


\section{Rhoades, * et al.}

$30 \mathrm{~min}$ at the same temperature, the reaction mixture was quenched by the addition of methanol $(15 \mu \mathrm{L}$, $0.38 \mathrm{mmol}, 1.0$ equiv) and a saturated aqueous solution of ammonium chloride ( $5 \mathrm{~mL})$, and allowed to warm to $22{ }^{\circ} \mathrm{C}$. The two phases were separated, and the aqueous layer was extracted with ethyl acetate (3 $\times 5 \mathrm{~mL}$ ). The combined organic phases were dried over anhydrous sodium sulfate and concentrated under reduced pressure. The obtained residue was purified by flash column chromatography (silica gel, $5 \rightarrow 15 \rightarrow 30 \%$ ethyl acetate in hexanes) to afford alcohol 19 (132 $\mathrm{mg}, 0.330 \mathrm{mmol}, 88 \%$ yield) as a colorless oil.

$\mathbf{R}_{f}=0.37$ (silica gel, 30\% ethyl acetate in hexanes, UV, $p$-anisaldehyde).

$[\boldsymbol{\alpha}]_{\mathbf{D}}^{22}=-291\left(c=0.80, \mathrm{CH}_{2} \mathrm{Cl}_{2}\right)$

FT-IR (thin film): $v_{\max } 3422,3068,3049,2998,2961,2928,2875,2855,1731,1713,1618,1445,1427$, $1376,1328,1247,1192,1155,1113,1079,1048,1027,1000,979.947,941,912,834,814,778,731$, $700 \mathrm{~cm}^{-1}$.

${ }^{1}$ H NMR (600 MHz, CDCl3): $\delta$ 7.53-7.49 (m, 2 H), 7.37-7.33 (m, 3 H), 6.07 (br dd, $J=10.1,8.9$ Hz, 1 H), 6.02 (br d, $J=8.9 \mathrm{~Hz}, 1 \mathrm{H}), 5.69$ (br s, $1 \mathrm{H}), 5.09$ (br dd, $J=10.3,10.1 \mathrm{~Hz}, 1 \mathrm{H}), 4.99$ (d, $J=10.2$ Hz, $1 \mathrm{H}), 3.90$ (ddddd, $J=10.2,8.0,7.8,5.8,3.6 \mathrm{~Hz}, 1 \mathrm{H}), 2.76(\mathrm{ddq}, J=10.3,10.2,6.8 \mathrm{~Hz}, 1 \mathrm{H}), 2.57$ $(\mathrm{ddd}, J=14.2,5.6,5.3 \mathrm{~Hz}, 1 \mathrm{H}), 2.56(\mathrm{dd}, J=15.2,3.6 \mathrm{~Hz}, 1 \mathrm{H}), 2.48(\mathrm{dd}, J=15.2,7.8 \mathrm{~Hz}, 1 \mathrm{H}), 2.47$ (br d, $J=8.0 \mathrm{~Hz}, 1 \mathrm{H}), 2.17$ (dddd, $J=13.9,13.5,5.8,5.6 \mathrm{~Hz}, 1 \mathrm{H}), 1.91$ (ddd, $J=14.2,13.9,4.1 \mathrm{~Hz}, 1$ H), 1.82 (br s, $3 \mathrm{H}), 1.69$ (br s, $3 \mathrm{H}), 1.59$ (dddd, $J=13.5,10.2,5.3,4.1 \mathrm{~Hz}, 1 \mathrm{H}), 0.89$ (d, J = 6.8 Hz, 3 H), 0.39 (s, $3 \mathrm{H}), 0.37$ (s, $3 \mathrm{H}) \mathrm{ppm}$.

${ }^{13}$ C NMR (151 MHz, CDCl $): \delta 170.7,151.1,139.2,138.0,133.8,132.3,129.1,128.6,128.5,128.0$, $122.1,84.9,67.9,42.5,34.9,34.7,29.3,27.7,16.9,16.7,-1.0,-1.2 \mathrm{ppm}$.

HRMS (ESI, $\boldsymbol{m} / z$ ): calcd for $\mathrm{C}_{24} \mathrm{H}_{34} \mathrm{O}_{3} \mathrm{SiNa}^{+}[\mathrm{M}+\mathrm{Na}]^{+} 421.2169$, found 421.2175, $\Delta 1.4$ ppm. 


\section{Rhoades, * et al.}

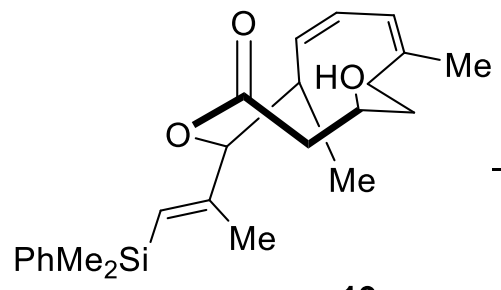

19

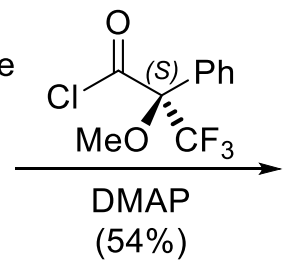

$(54 \%)$

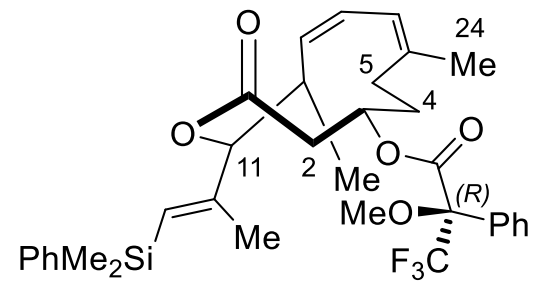

$(R)-S 13$

$(4 S, 7 Z, 9 Z, 11 S, 12 S)-12-\{(E)-1-[D i m e t h y l(p h e n y l) s i l y l] p r o p-1-e n-2-y l\}-7,11-d i m e t h y l-2-$

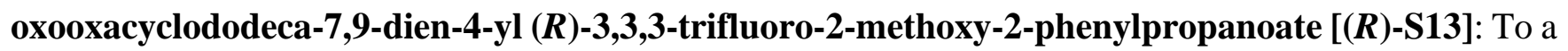
stirred solution of alcohol $19\left(6.0 \mathrm{mg}, 15 \mu \mathrm{mol}, 1.0\right.$ equiv) in dichloromethane $(1.0 \mathrm{~mL})$ at $22{ }^{\circ} \mathrm{C}$ was added (S)-(+)- $\alpha$-methoxy- $\alpha$-(trifluoromethyl)phenylacetyl chloride (8.0 $\mu \mathrm{L}, 45 \mu$ mol, 3.0 equiv), followed by 4-dimethylaminopyridine $(7.3 \mathrm{mg}, 60 \mu \mathrm{mol}, 4.0$ equiv). After stirring for $4 \mathrm{~h}$, the reaction mixture was concentrated under reduced pressure and purified directly by preparative thin layer chromatography $(10 \%$ ethyl acetate in hexanes) to afford Mosher ester $(\boldsymbol{R})-\mathbf{S 1 3}(5.0 \mathrm{mg}, 8.1 \mu \mathrm{mol}, 54 \%$ yield $)$ as a colorless oil. $\mathbf{R}_{f}=0.36$ (silica gel, $10 \%$ ethyl acetate in hexanes, UV, $p$-anisaldehyde).

$[\boldsymbol{\alpha}]_{\mathbf{D}}^{22}=-174\left(c=0.80, \mathrm{CH}_{2} \mathrm{Cl}_{2}\right)$.

FT-IR (thin film): $v_{\max }$ 3069, 2999, 2961, 2929, 2876, 2851, 1736, 1618, 1488, 1451, 1428, 1403, 1378, 1326, 1247, 1238, 1185, 1168, 1114, 1081, 1068, 1020, 980, 930, 899, 877, 832, 814, 766, 731, $700 \mathrm{~cm}^{-1}$.

${ }^{1}$ H NMR (600 MHz, CDCl3): $\delta$ 7.53-7.50 (m, 4 H), 7.42-7.37 (m, 3 H), 7.36-7.33 (m, 3 H), 6.09 (br dq, $J=7.6,1.5 \mathrm{~Hz}, 1 \mathrm{H}), 6.02(\mathrm{dd}, J=10.4,7.6 \mathrm{~Hz}, 1 \mathrm{H}), 5.71($ br s, $1 \mathrm{H}), 5.37$ (dddd, $J=10.1,9.9,4.1,2.9$ Hz, $1 \mathrm{H}), 5.11(\mathrm{ddd}, J=10.5,10.4,1.5 \mathrm{~Hz}, 1 \mathrm{H}), 4.94(\mathrm{~d}, J=10.3 \mathrm{~Hz}, 1 \mathrm{H}), 3.55$ (s, $3 \mathrm{H}), 2.80$ (ddq, $J=$ 10.5, 10.3, $6.7 \mathrm{~Hz}, 1 \mathrm{H}), 2.79$ (dd, $J=15.3,4.1 \mathrm{~Hz}, 1 \mathrm{H}), 2.50(\mathrm{ddd}, J=14.6,5.3,4.4 \mathrm{~Hz}, 1 \mathrm{H}), 2.34$ (dd, $J=15.3,9.9 \mathrm{~Hz}, 1 \mathrm{H}), 2.16(\mathrm{dddd}, J=14.5,11.7,5.3,2.9 \mathrm{~Hz}, 1 \mathrm{H}), 1.85(\mathrm{ddd}, J=14.6,11.7,4.3 \mathrm{~Hz}, 1$ H), 1.79 (br d, $J=1.5 \mathrm{~Hz}, 3 \mathrm{H}), 1.76$ (dddd, $J=14.5,10.1,4.4,4.3 \mathrm{~Hz}, 1 \mathrm{H}), 1.73(\mathrm{~d}, J=0.6 \mathrm{~Hz}, 3 \mathrm{H})$, $0.89(\mathrm{~d}, J=6.7 \mathrm{~Hz}, 3 \mathrm{H}), 0.40(\mathrm{~s}, 3 \mathrm{H}), 0.37$ (s, $3 \mathrm{H}) \mathrm{ppm}$. 


\section{Rhoades, * et al.}

${ }^{13}$ C NMR (151 MHz, CDCl $): \delta 167.9,166.4,150.8,139.2,137.7,133.8,133.3,132.1,129.8,129.1$, 128.9, 128.6, 128.2, 128.0, 127.3, $123.4(\mathrm{q}, J=288.8 \mathrm{~Hz}), 122.3,85.5,84.7(\mathrm{q}, J=27.4 \mathrm{~Hz}), 71.3,55.6$, $40.3,35.1,31.1,28.5,26.7,16.7,16.6,-1.0,-1.2 \mathrm{ppm}$.

${ }^{19}$ F NMR (565 MHz, CDCl3): $\delta-70.5$ ppm.

HRMS (ESI, $\boldsymbol{m} / \mathbf{z}$ ): calcd for $\mathrm{C}_{34} \mathrm{H}_{41} \mathrm{~F}_{3} \mathrm{O}_{5} \mathrm{SiNa}^{+}[\mathrm{M}+\mathrm{Na}]^{+}$637.2568, found 637.2566, $\Delta 0.3 \mathrm{ppm}$.

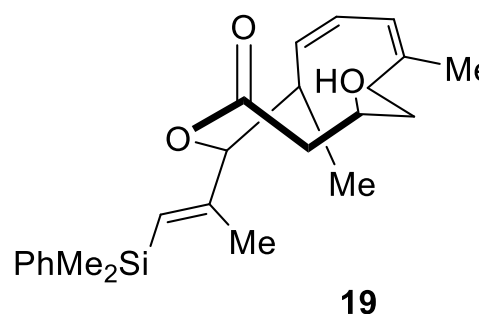

19

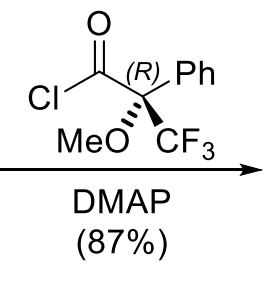

$(87 \%)$

\section{$(4 S, 7 Z, 9 Z, 11 S, 12 S)-12-\{(E)-1-[D i m e t h y l(p h e n y l)$ silyl]prop-1-en-2-yl\}-7,11-dimethyl-2-}

oxooxacyclododeca-7,9-dien-4-yl $(S)-3,3,3-t r i f l u o r o-2-m e t h o x y-2-p h e n y l p r o p a n o a t e ~[(S)-S 13]$ : To a stirred solution of alcohol $19\left(6.0 \mathrm{mg}, 15 \mu \mathrm{mol}, 1.0\right.$ equiv) in dichloromethane $(1.0 \mathrm{~mL})$ at $22{ }^{\circ} \mathrm{C}$ was added (R)-(-)- $\alpha$-methoxy- $\alpha$-(trifluoromethyl)phenylacetyl chloride $(8.0 \mu \mathrm{L}, 45 \mu \mathrm{mol}, 3.0$ equiv), followed by 4-dimethylaminopyridine ( $7.3 \mathrm{mg}, 60 \mu \mathrm{mol}, 4.0$ equiv). After stirring for $4 \mathrm{~h}$, the reaction mixture was concentrated under reduced pressure and purified directly by preparative thin layer chromatography (10\% ethyl acetate in hexanes) to afford Mosher ester $(\boldsymbol{S})$-S13 $(8.0 \mathrm{mg}, 13 \mu \mathrm{mol}, 87 \%$ yield) as a colorless oil.

$\mathbf{R}_{f}=0.36$ (silica gel, 10\% ethyl acetate in hexanes, UV, $p$-anisaldehyde).

$[\boldsymbol{\alpha}]_{\mathbf{D}}^{22}=-178\left(c=0.50, \mathrm{CH}_{2} \mathrm{Cl}_{2}\right)$.

FT-IR (thin film): $v_{\max } 3067,2964,2927,2873,2858,1736,1619,1497,1488,1452,1428,1378,1238$, $1184,1168,1114,1080,1062,1032,1019,981,932,921,900,833,814,777,731,699 \mathrm{~cm}^{-1}$.

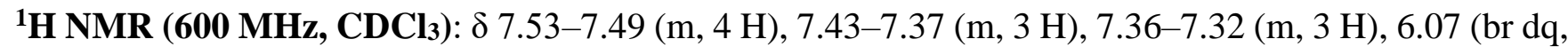
$J=7.6 \mathrm{~Hz}, 1.4 \mathrm{~Hz}, 1 \mathrm{H}), 6.01(\mathrm{dd}, J=10.6,7.6 \mathrm{~Hz}, 1 \mathrm{H}), 5.71(\mathrm{br} \mathrm{s}, 1 \mathrm{H}), 5.34$ (dddd, $J=10.1,6.7,4.1$, $2.7 \mathrm{~Hz}, 1 \mathrm{H}), 5.10(\mathrm{ddd}, J=10.6,10.4,1.5 \mathrm{~Hz}, 1 \mathrm{H}), 4.94(\mathrm{~d}, J=10.3 \mathrm{~Hz}, 1 \mathrm{H}), 3.52(\mathrm{~s}, 3 \mathrm{H}), 2.91(\mathrm{dd}, J$ 


\section{Rhoades, * et al.}

$=15.2,4.1 \mathrm{~Hz}, 1 \mathrm{H}), 2.81(\mathrm{ddq}, J=10.4,10.3,6.7 \mathrm{~Hz}, 1 \mathrm{H}), 2.44(\mathrm{dd}, J=15.2,10.1 \mathrm{~Hz}, 1 \mathrm{H}), 2.43-2.39$

(m, $1 \mathrm{H}), 2.15$ (dddd, 14.0, 11.6, 6.7, $4.3 \mathrm{~Hz}, 1 \mathrm{H}), 1.76$ (br d, $J=1.4 \mathrm{~Hz}, 3 \mathrm{H}), 1.74-1.68$ (m, $2 \mathrm{H}), 1.71$

$(\mathrm{d}, J=0.7 \mathrm{~Hz}, 3 \mathrm{H}), 0.89(\mathrm{~d}, J=6.7 \mathrm{~Hz}, 3 \mathrm{H}), 0.39(\mathrm{~s}, 3 \mathrm{H}), 0.37(\mathrm{~s}, 3 \mathrm{H}), \mathrm{ppm}$.

${ }^{13}$ C NMR (151 MHz, CDCl3): $\delta$ 167.9, 166.3, 150.8, 139.2, 137.9, 133.8, 133.1, 132.2, 129.9, 129.1, 129.0, 128.6, 128.3, 128.0, 127.3, $123.5(\mathrm{q}, J=288.4 \mathrm{~Hz}), 122.1,85.6,84.4(\mathrm{q}, J=27.4 \mathrm{~Hz}), 71.4,55.5$, $40.5,35.1,31.1,28.3,26.8,16.7,16.6,-1.0,-1.2 \mathrm{ppm}$.

${ }^{19}$ F NMR (565 MHz, CDCl3): $\delta-70.2$ ppm.

HRMS (ESI, $m / z$ ): calcd for $\mathrm{C}_{34} \mathrm{H}_{41} \mathrm{~F}_{3} \mathrm{O}_{5} \mathrm{SiNa}^{+}[\mathrm{M}+\mathrm{Na}]^{+}$637.2568, found 637.2560, $\Delta 1.3 \mathrm{ppm}$.

Table S6. Mosher ester analysis of MTPA esters derived from alcohol 19.

\begin{tabular}{|c|c|c|c|}
\hline Proton & $\begin{array}{c}\text { Chemical shift of }(S)- \\
\text { MTPA derivative }\end{array}$ & $\begin{array}{c}\text { Chemical shift of }(R)- \\
\text { MTPA derivative }\end{array}$ & $\begin{array}{c}\Delta \delta:(S)-\mathrm{MTPA}- \\
(R)-\mathrm{MTPA}\end{array}$ \\
\hline $\mathbf{2 4}$ & 1.76 & 1.79 & -0.03 \\
\hline $\mathbf{5 a}$ & 2.42 & 2.50 & -0.08 \\
\hline $\mathbf{5 b}$ & 2.15 & 2.16 & -0.01 \\
\hline $\mathbf{4 a}$ & 1.73 & 1.85 & -0.12 \\
\hline $\mathbf{4 b}$ & 1.69 & 1.76 & -0.07 \\
\hline $\mathbf{2 a}$ & 2.91 & 2.79 & +0.12 \\
\hline $\mathbf{2 b}$ & 2.44 & 2.34 & +0.10 \\
\hline $\mathbf{1 1}$ & 4.94 & 4.94 & \pm 0.00 \\
\hline
\end{tabular}

On the basis of the Mosher ester analysis, ${ }^{21}$ the absolute configuration at C3 (pladienolide numbering) is unambiguously assigned as $(R)$. 
D. Rhoades, * et al.

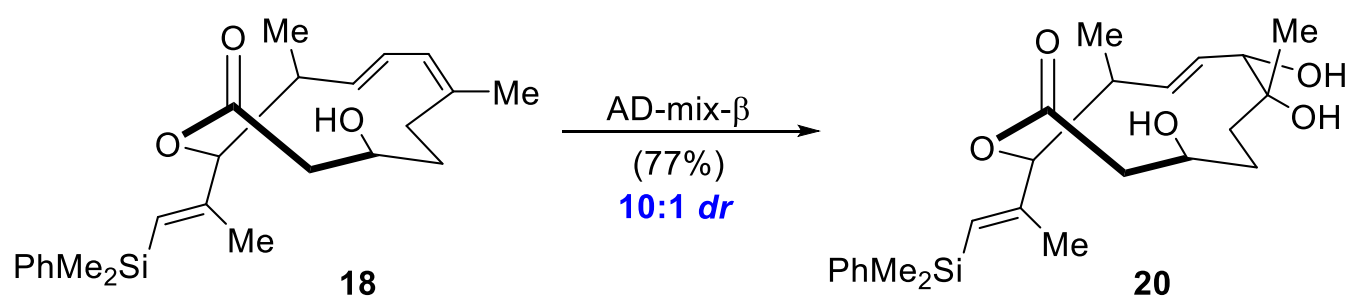

$(4 R, 7 R, 8 S, 11 S, 12 S, E)-12-\{(E)-1-[D i m e t h y l(p h e n y l) \operatorname{silyl}]$ prop-1-en-2-yl\}-4,7,8-trihydroxy-7,11-

dimethyloxacyclododec-9-en-2-one (20): To a vigorously stirred solution of alcohol 18 (232 mg, 0.582 mmol, 1.0 equiv) in $1: 1$ tert-butanol/water $(6.4 \mathrm{~mL})$ at $0{ }^{\circ} \mathrm{C}$ was added methanesulfonamide $(56.0 \mathrm{mg}$, $0.582 \mathrm{mmol}, 1.0$ equiv) and AD-mix- $\beta$ (736 mg).* After $24 \mathrm{~h}$, additional AD-mix- $\beta$ (184 mg) was added, and the reaction mixture was stirred for another $24 \mathrm{~h}$. Then the reaction mixture was quenched with a saturated aqueous solution of sodium thiosulfate $(6 \mathrm{~mL})$ and diluted with ethyl acetate $(15 \mathrm{~mL})$. The two phases were separated, and the aqueous layer was extracted with ethyl acetate $(3 \times 5 \mathrm{~mL})$. The combined organic phases were washed with an aqueous solution of potassium hydroxide $(2.0 \mathrm{M}, 2 \times 5 \mathrm{~mL})$, dried over anhydrous sodium sulfate, and concentrated under reduced pressure. The obtained residue was purified by flash column chromatography (silica gel, $50 \rightarrow 25 \%$ hexanes in ethyl acetate) to afford unreacted alcohol $18(19.0 \mathrm{mg}, 47.7 \mu \mathrm{mol})$ and triols $\mathbf{2 0}(176 \mathrm{mg}, 0.407 \mathrm{mmol})$ and 21 (17.6 mg, 40.7 $\mu \mathrm{mol}$ ) as colorless oils (77\% overall yield, $85 \%$ brsm, 10:1 dr). Recrystallization of $\mathbf{2 0}$ from ca. 10:1 dichloromethane/hexanes provided crystals suitable for X-ray crystallographic analysis. *NOTE: This reaction was set up in a temperature-controlled cold room $\left(4{ }^{\circ} \mathrm{C}\right)$ to adequately maintain its reaction temperature for extended time periods.

$\mathbf{R}_{f}=0.25$ (silica gel, 25\% hexanes in ethyl acetate, UV, $p$-anisaldehyde).

m.p. $=148-151^{\circ} \mathrm{C}$.

$[\boldsymbol{\alpha}]_{\mathbf{D}}^{22}=-51.0\left(c=0.50, \mathrm{CH}_{2} \mathrm{Cl}_{2}\right)$.

FT-IR (thin film): $v_{\max } 3407,3068,3046,2956,2925,2872,2855,1708,1669,1616,1554,1487,1458$, $1428,1371,1340,1249,1214,1175,1113,1080,1049,1022,978,942,906,839,815,787,731,700$, $667 \mathrm{~cm}^{-1}$. 


\section{Rhoades, * et al.}

${ }^{1} \mathbf{H}$ NMR (600 MHz, CDCl3): $\delta$ 7.50-7.49 (m, $\left.2 \mathrm{H}\right), 7.37-7.33(\mathrm{~m}, 3 \mathrm{H}), 5.73$ (br q, $J=1.0 \mathrm{~Hz}, 1 \mathrm{H}$ ), $5.71(\mathrm{dd}, J=15.2,9.7 \mathrm{~Hz}, 1 \mathrm{H}), 5.42(\mathrm{dd}, J=15.2,9.9 \mathrm{~Hz}, 1 \mathrm{H}), 5.16(\mathrm{~d}, J=10.6 \mathrm{~Hz}, 1 \mathrm{H}), 3.81(\mathrm{dd}, J=$ 9.7, 3.9 Hz, $1 \mathrm{H}), 3.77-3.72(\mathrm{~m}, 1 \mathrm{H}), 3.53(\mathrm{~d}, J=10.9 \mathrm{~Hz}, 1 \mathrm{H}), 2.65$ (br dd, $J=15.1,3.8 \mathrm{~Hz}, 1 \mathrm{H}), 2.55$ (dd, $J=15.1,3.0 \mathrm{~Hz}, 1 \mathrm{H}), 2.45(\mathrm{ddq}, J=10.6,9.9,6.7 \mathrm{~Hz}, 1 \mathrm{H}), 2.37(\mathrm{br} \mathrm{s}, 1 \mathrm{H}), 1.81(\mathrm{br} \mathrm{d}, J=3.9 \mathrm{~Hz}$, $1 \mathrm{H}), 1.68(\operatorname{app~tt}, J=13.9,3.9 \mathrm{~Hz}, 1 \mathrm{H}), 1.67(\mathrm{~d}, J=0.7 \mathrm{~Hz}, 3 \mathrm{H}), 1.47(\operatorname{app~td}, J=12.8,3.5 \mathrm{~Hz}, 1 \mathrm{H})$, $1.39(\operatorname{app} t d, J=12.8,4.5 \mathrm{~Hz}, 1 \mathrm{H}), 1.31(\mathrm{br} \mathrm{s}, 3 \mathrm{H}), 1.27(\operatorname{app} t \mathrm{tt}, J=13.9,2.0 \mathrm{~Hz}, 1 \mathrm{H}), 0.95(\mathrm{~d}, J=6.7$ Hz, $3 \mathrm{H}), 0.39$ (s, $3 \mathrm{H}), 0.37$ (s, $3 \mathrm{H}) \mathrm{ppm}$.

${ }^{13}$ C NMR (151 MHz, CDCl $): \delta 172.4,150.2,139.0,137.5,133.8,130.3,129.7,129.1,128.0,84.4,77.4$, $73.5,69.4,40.9,38.4,35.7,29.9,24.6,16.9,16.8,-1.1,-1.3 \mathrm{ppm}$.

HRMS (ESI, $\boldsymbol{m} / \boldsymbol{z}$ ): calcd for $\mathrm{C}_{24} \mathrm{H}_{36} \mathrm{O}_{5} \mathrm{SiNa}^{+}[\mathrm{M}+\mathrm{Na}]^{+} 455.2224$, found 455.2220, $\Delta 0.9$ ppm.

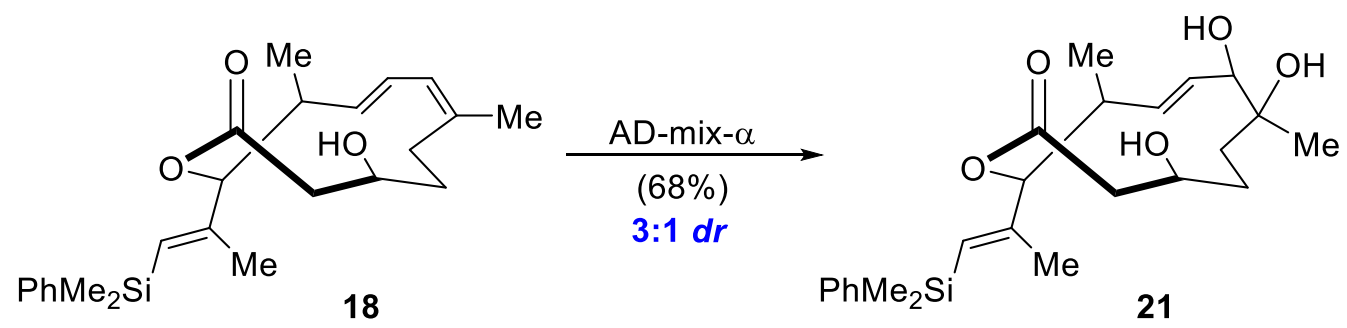

\section{$(4 R, 7 S, 8 R, 11 S, 12 S, E)-12-\{(E)-1-[D i m e t h y l(p h e n y l)$ silyl]prop-1-en-2-yl $\}-4,7,8-t r i h y d r o x y-7,11-$}

dimethyloxacyclododec-9-en-2-one (21): To a vigorously stirred solution of alcohol $\mathbf{1 8}$ (84.0 mg, 0.210 mmol, 1.0 equiv) in 1:1 tert-butanol/water $(2.3 \mathrm{~mL})$ at $0{ }^{\circ} \mathrm{C}$ was added methanesulfonamide $(20.2 \mathrm{mg}$, $0.210 \mathrm{mmol}, 1.0$ equiv) and AD-mix- $\alpha(357 \mathrm{mg})$.* After $48 \mathrm{~h}$, the reaction mixture was quenched with a saturated aqueous solution of sodium thiosulfate $(2 \mathrm{~mL})$ and diluted with ethyl acetate $(5 \mathrm{~mL})$. The two phases were separated, and the aqueous layer was extracted with ethyl acetate $(3 \times 3 \mathrm{~mL})$. The combined organic phases were washed with an aqueous solution of potassium hydroxide $(2.0 \mathrm{M}, 2 \times 5 \mathrm{~mL})$, dried over anhydrous sodium sulfate, and concentrated under reduced pressure. The obtained residue was purified by flash column chromatography (silica gel, $50 \rightarrow 25 \%$ hexanes in ethyl acetate) to afford triol 20 (15.4 mg, $35.7 \mu \mathrm{mol})$ and triol $21(46.3 \mathrm{mg}, 0.107 \mathrm{mmol})$ as colorless oils (68\% overall yield, 3:1 $\mathrm{dr}$ ). 


\section{Rhoades, * et al.}

*NOTE: This reaction was set up in a temperature-controlled cold room $\left(4^{\circ} \mathrm{C}\right)$ to adequately maintain its reaction temperature for extended time periods.

$\mathbf{R}_{f}=0.38$ (silica gel, $40 \%$ ethyl acetate in hexanes, UV, $p$-anisaldehyde).

$[\boldsymbol{\alpha}]_{\mathbf{D}}^{22}=-4.6\left(c=0.70, \mathrm{CH}_{2} \mathrm{Cl}_{2}\right)$.

FT-IR (thin film): $v_{\max } 3460,3069,3046,2961,2929,2873,2855,1731,1717,1671,1617,1486,1456$, $1428,1409,1378,1326,1306,1248,1177,1156,1113,1084,1064,1031,974,941,914,886,839,816$, $797,781,731,701 \mathrm{~cm}^{-1}$.

${ }^{1}$ H NMR (600 MHz, CDCl 3$): \delta 7.52-7.49$ (m, $\left.2 \mathrm{H}\right), 7.37-7.33(\mathrm{~m}, 3 \mathrm{H}), 5.75$ (br s, $\left.1 \mathrm{H}\right), 5.51(\mathrm{dd}, J=$ 16.1, 8.7 Hz, $1 \mathrm{H}), 5.40(\mathrm{dd}, J=16.1,6.1 \mathrm{~Hz}, 1 \mathrm{H}), 4.92(\mathrm{~d}, J=10.5 \mathrm{~Hz}, 1 \mathrm{H}), 3.80-3.76(\mathrm{~m}, 1 \mathrm{H}), 3.45$ $(\mathrm{d}, J=10.6 \mathrm{~Hz}, 1 \mathrm{H}), 3.22(\mathrm{~d}, J=6.1 \mathrm{~Hz}, 1 \mathrm{H}), 2.67(\mathrm{dd}, J=15.4,4.5 \mathrm{~Hz}, 1 \mathrm{H}), 2.58(\mathrm{dd}, J=15.4,3.0$ Hz, $1 \mathrm{H}), 2.41$ (ddq, $J=10.5,8.7,6.9 \mathrm{~Hz}, 1 \mathrm{H}), 1.88-1.78(\mathrm{~m}, 2 \mathrm{H}), 1.64$ (br s, $3 \mathrm{H}), 1.59$ (br s, $2 \mathrm{H})$, 1.40-1.34 (m, 1 H), 1.33 (br s, $3 \mathrm{H}), 1.10-1.05(\mathrm{~m}, 1 \mathrm{H}), 0.95$ (d, J = 6.9 Hz, $3 \mathrm{H}), 0.39$ (s, $3 \mathrm{H}), 0.37$ (s, $3 \mathrm{H}) \mathrm{ppm}$.

${ }^{13}$ C NMR (151 MHz, CDCl 3$): \delta 172.0,150.4,139.0,135.5,133.8,130.3,129.4,129.1,128.0,84.2,69.0$, $62.8,62.0,39.5,38.7,32.6,30.6,21.6,16.8,16.5,-1.1,-1.2 \mathrm{ppm}$.

HRMS (ESI, $\boldsymbol{m} / \boldsymbol{z}$ ): calcd for $\mathrm{C}_{24} \mathrm{H}_{36} \mathrm{O}_{5} \mathrm{SiNa}^{+}[\mathrm{M}+\mathrm{Na}]^{+} 455.2224$, found 455.2220, $\Delta 0.9$ ppm.

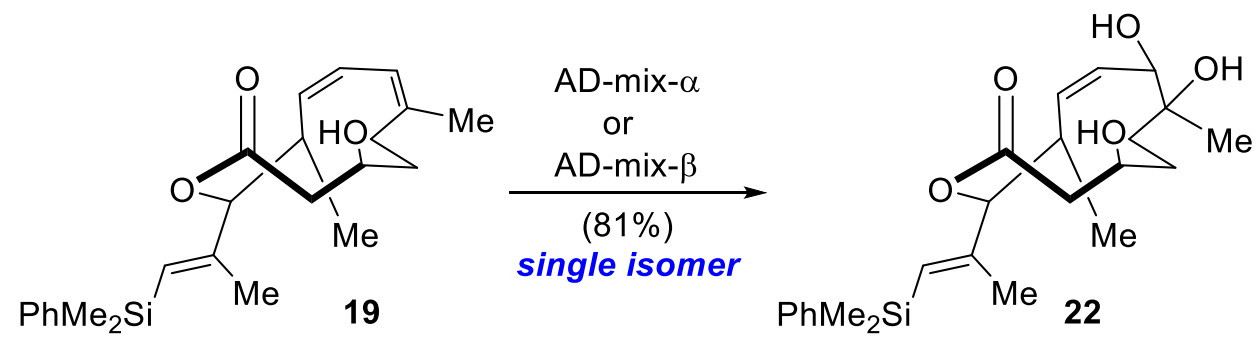

$(4 R, 7 S, 8 R, 11 S, 12 S, Z)-12-\{(E)-1-[D i m e t h y l(p h e n y l) s i l y l] p r o p-1-e n-2-y l\}-4,7,8-t r i h y d r o x y-7,11-$

dimethyloxacyclododec-9-en-2-one (22): To a vigorously stirred solution of alcohol 19 (66.0 mg, 0.166 mmol, 1.0 equiv) in $1: 1$ tert-butanol/water $(1.8 \mathrm{~mL})$ at $0{ }^{\circ} \mathrm{C}$ was added methanesulfonamide $(15.8 \mathrm{mg}$, $0.166 \mathrm{mmol}, 1.0$ equiv) and $\mathrm{AD}-\mathrm{mix}-\alpha$ or $\mathrm{AD}-\mathrm{mix}-\beta(210 \mathrm{mg}) . *$ After $48 \mathrm{~h}$, the reaction mixture was 


\section{Rhoades, * et al.}

quenched with a saturated aqueous solution of sodium thiosulfate $(1.5 \mathrm{~mL})$ and diluted with ethyl acetate $(5 \mathrm{~mL})$. The two phases were separated, and the aqueous layer was extracted with ethyl acetate $(3 \times 3$ $\mathrm{mL})$. The combined organic phases were washed with an aqueous solution of potassium hydroxide (2.0 $\mathrm{M}, 2 \times 5 \mathrm{~mL}$ ), dried over anhydrous sodium sulfate, and concentrated under reduced pressure. The obtained residue was purified by flash column chromatography (silica gel, $50 \rightarrow 0 \%$ hexanes in ethyl acetate) to afford triol $22(58.0 \mathrm{mg}, 0.134 \mathrm{mmol}, 81 \%$ yield $)$ as a colorless oil. *NOTE: This reaction was set up in a temperature-controlled cold room $\left(4^{\circ} \mathrm{C}\right)$ to adequately maintain its reaction temperature for extended time periods. Identical yields were obtained when using AD-mix- $\alpha$ or AD-mix- $\beta$.

$\mathbf{R}_{f}=0.34$ (silica gel, ethyl acetate, UV, $p$-anisaldehyde).

$[\boldsymbol{\alpha}]_{\mathbf{D}}^{22}=-18.4\left(c=1.00, \mathrm{CH}_{2} \mathrm{Cl}_{2}\right)$.

FT-IR (thin film): $v_{\max } 3402,3068,3049,2965,2876,1728,1660,1618,1487,1452,1427,1373,1332$, $1286,1248,1167,1114,1057,1032,1005,980,966,933,894,836,779,731,700,667 \mathrm{~cm}^{-1}$.

${ }^{1} \mathbf{H}$ NMR (600 MHz, CDCl $): \delta 7.52-7.49(\mathrm{~m}, 2 \mathrm{H}), 7.37-7.33(\mathrm{~m}, 3 \mathrm{H}), 5.72($ br q, $J=0.7 \mathrm{~Hz}, 1 \mathrm{H})$, 5.70 (br dd, $J=10.8,10.4 \mathrm{~Hz}, 1 \mathrm{H}), 5.38$ (br dd, $J=10.8,10.5 \mathrm{~Hz}, 1 \mathrm{H}), 5.10(\mathrm{~d}, J=10.1 \mathrm{~Hz}, 1 \mathrm{H}), 4.10$ $(\mathrm{d}, J=10.4 \mathrm{~Hz}, 1 \mathrm{H}), 3.95-3.91(\mathrm{~m}, 1 \mathrm{H}), 2.79(\mathrm{ddq}, J=10.5,10.1,6.7 \mathrm{~Hz}, 1 \mathrm{H}), 2.61(\mathrm{dd}, J=15.2,4.4$ $\mathrm{Hz}, 1 \mathrm{H}), 2.58(\mathrm{dd}, J=15.2,6.1 \mathrm{~Hz}, 1 \mathrm{H}), 2.37$ (br s, $1 \mathrm{H}), 2.20($ br s, $1 \mathrm{H}), 1.93($ app tt, $J=13.7,4.1 \mathrm{~Hz}$, $1 \mathrm{H}), 1.86$ (br s, $1 \mathrm{H}), 1.75$ (app tdd, $J=13.7,8.2,4.5 \mathrm{~Hz}, 1 \mathrm{H}), 1.68$ (d, $J=0.7 \mathrm{~Hz}, 3 \mathrm{H}), 1.56$ (app td, $J$ $=13.6,4.0 \mathrm{~Hz}, 1 \mathrm{H}), 1.47(\operatorname{app~td}, J=13.6,4.4 \mathrm{~Hz}, 1 \mathrm{H}), 1.33(\mathrm{br} \mathrm{s}, 3 \mathrm{H}), 0.90(\mathrm{~d}, J=6.7 \mathrm{~Hz}, 3 \mathrm{H}), 0.39$ (s, $3 \mathrm{H}), 0.38$ (s, $3 \mathrm{H}) \mathrm{ppm}$.

${ }^{13}$ C NMR (151 MHz, CDCl $): ~ \delta 170.0,150.4,139.0,135.9,133.8,129.5,129.3,129.1,128.0,85.2,73.7$, 72.4, 68.1, 40.0, 35.3, 33.7, 30.0, 27.5, 17.5, 16.9, -1.1, -1.2 ppm.

HRMS (ESI, $\boldsymbol{m} / \boldsymbol{z}$ ): calcd for $\mathrm{C}_{24} \mathrm{H}_{36} \mathrm{O}_{5} \mathrm{SiNa}^{+}[\mathrm{M}+\mathrm{Na}]^{+} 455.2224$, found $455.2228, \Delta 0.9 \mathrm{ppm}$. 
D. Rhoades, ${ }^{*}$ et al.

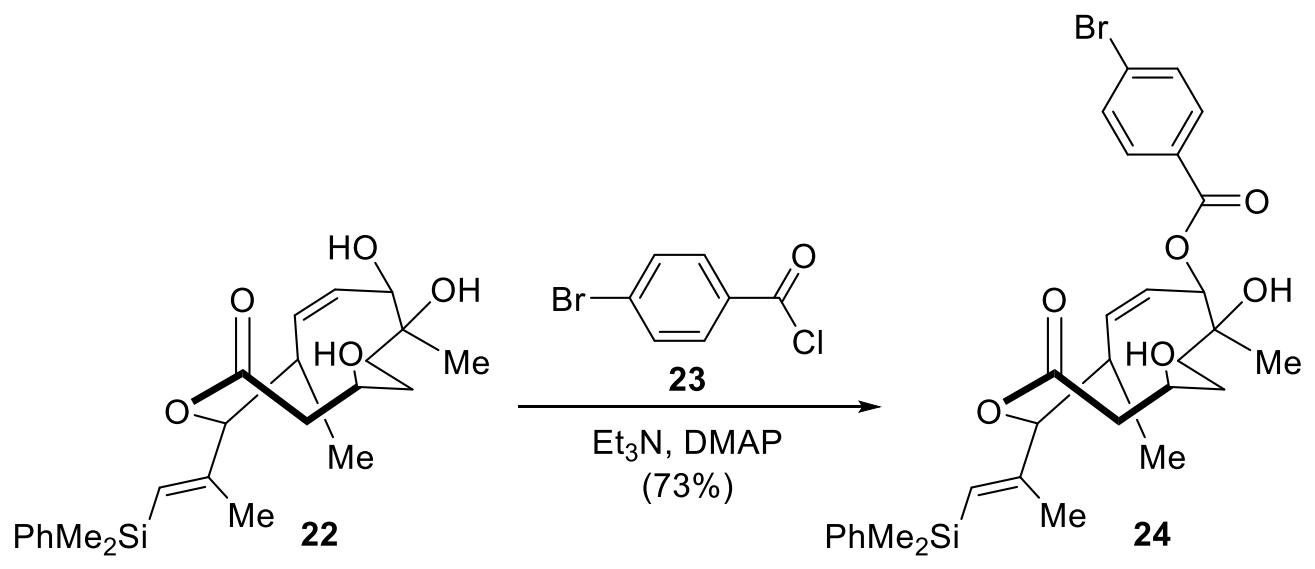

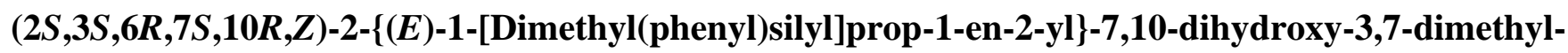

12-oxooxacyclododec-4-en-6-yl 4-bromobenzoate (24): To a stirred solution of alcohol 22 (9.1 mg, 21

$\mu \mathrm{mol}, 1.0$ equiv) in dichloromethane $(0.6 \mathrm{~mL})$ at $0{ }^{\circ} \mathrm{C}$ was added triethylamine $(9.0 \mu \mathrm{L}, 63 \mu \mathrm{mol}, 3.0$ equiv), 4-bromobenzoyl chloride (23) (7.0 mg, $32 \mu \mathrm{mol}, 1.5$ equiv), and 4-dimethylaminopyridine ( 0.77 $\mathrm{mg}, 6.3 \mu \mathrm{mol}, 0.3$ equiv), and the reaction mixture was allowed to warm to $22^{\circ} \mathrm{C}$. After stirring for $1 \mathrm{~h}$, the reaction mixture was concentrated under reduced pressure and purified directly by preparative thin layer chromatography (15\% hexanes in ethyl acetate) to afford ester $\mathbf{2 4}(9.42 \mathrm{mg}, 15.3 \mu \mathrm{mol}, 73 \%$ yield) as a white amorphous solid. Recrystallization from ca. 10:1 dichloromethane/hexanes provided crystals suitable for X-ray crystallographic analysis.

$\mathbf{R}_{f}=0.33$ (silica gel, 30\% hexanes in ethyl acetate, UV, $p$-anisaldehyde).

m.p. $=185-190{ }^{\circ} \mathrm{C}$.

$[\boldsymbol{\alpha}]_{\mathbf{D}}^{22}=+66.7\left(c=0.60, \mathrm{CH}_{2} \mathrm{Cl}_{2}\right)$.

FT-IR (thin film): $v_{\max } 3351,3070,3048,2963,2941,2898,2856,1732,1720,1621,1592,1486,1453$, $1427,1398,1381,1360,1330,1287,1271,1248,1191,1176,1146,1103,1070,1024,1013,967,936$, $875,836,814,781,758,728,700 \mathrm{~cm}^{-1}$.

${ }^{1}$ H NMR (600 MHz, CDCl3): $\delta$ 7.89-7.87 (m, 2 H), 7.61-7.58 (m, 2 H), 7.54-7.51 (m, 2 H), 7.38-7.34 (m, $3 \mathrm{H}), 5.76$ (br dd, $J=11.0,10.5 \mathrm{~Hz}, 1 \mathrm{H}), 5.74(\mathrm{br} \mathrm{q}, J=0.6 \mathrm{~Hz}, 1 \mathrm{H}), 5.53(\mathrm{~d}, J=10.5 \mathrm{~Hz}, 1 \mathrm{H}), 5.48$ (br dd, $J=11.0,10.7 \mathrm{~Hz}, 1 \mathrm{H}), 5.14(\mathrm{~d}, J=10.3 \mathrm{~Hz}, 1 \mathrm{H}), 4.00-3.95(\mathrm{~m}, 1 \mathrm{H}), 3.11$ (ddq, $J=10.7,10.3$, 


\section{Rhoades, * et al.}

$6.6 \mathrm{~Hz}, 1 \mathrm{H}), 2.65(\mathrm{dd}, J=15.2,4.3 \mathrm{~Hz}, 1 \mathrm{H}), 2.62(\mathrm{dd}, J=15.2,6.2 \mathrm{~Hz}, 1 \mathrm{H}), 2.18(\mathrm{~d}, J=7.7 \mathrm{~Hz}, 1 \mathrm{H})$, $2.05(\operatorname{app~tt}, J=13.7,4.0 \mathrm{~Hz}, 1 \mathrm{H}), 1.87-1.80(\mathrm{~m}, 1 \mathrm{H}), 1.85($ br s, $1 \mathrm{H}), 1.76(\mathrm{~d}, J=0.6 \mathrm{~Hz}, 3 \mathrm{H}), 1.68$ $(\operatorname{app} t d, J=13.5,4.3 \mathrm{~Hz}, 1 \mathrm{H}), 1.58(\operatorname{app~td}, J=13.7,4.2 \mathrm{~Hz}, 1 \mathrm{H}), 1.32(\mathrm{br} \mathrm{s}, 3 \mathrm{H}), 0.90(\mathrm{~d}, J=6.6 \mathrm{~Hz}$ $3 \mathrm{H}), 0.40$ (s, $3 \mathrm{H}), 0.39$ (s, $3 \mathrm{H}) \mathrm{ppm}$.

${ }^{13}$ C NMR (151 MHz, CDCl3): $\delta$ 169.9, 164.8, 150.6, 139.2, 139.0, 133.8, 132.0, 131.2, 129.29, 129.25, $129.1,128.4,128.0,124.8,85.4,75.9,73.5,68.1,40.0,36.1,34.0,30.2,27.8,17.3,17.0,-1.0,-1.2$ ppm. HRMS (ESI, $\boldsymbol{m} / z)$ ): calcd for $\mathrm{C}_{31} \mathrm{H}_{39} \mathrm{BrO}_{6} \mathrm{SiNa}^{+}[\mathrm{M}+\mathrm{Na}]^{+}$637.1591, found 637.1592, $\Delta 0.2 \mathrm{ppm}$.

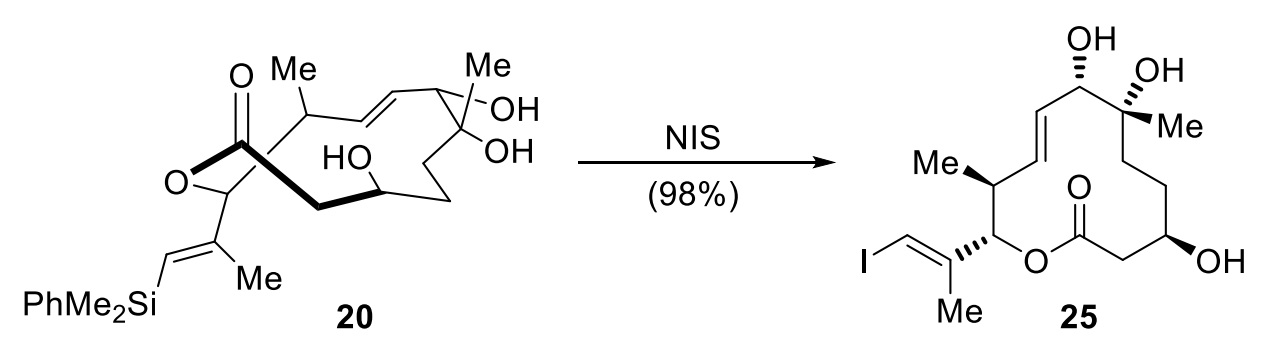

\section{$(4 R, 7 R, 8 S, 11 S, 12 S, E)-4,7,8-T r i h y d r o x y-12-[(E)-1$-iodoprop-1-en-2-yl]-7,11-}

dimethyloxacyclododec-9-en-2-one (25): To a stirred solution of vinyl silane 20 (78.0 mg, 0.180 mmol, 1.0 equiv) in 1,1,1,3,3,3-hexafluoro-2-propanol $(2.3 \mathrm{~mL})$ at $0{ }^{\circ} \mathrm{C}$ was added $N$-iodosuccinimide $(120 \mathrm{mg}$, $0.54 \mathrm{mmol}, 3.0$ equiv), at which point the color of the reaction mixture changed from colorless to pink. After stirring for $30 \mathrm{sec}$, the reaction mixture was quenched with a saturated aqueous solution of sodium thiosulfate $(2.0 \mathrm{~mL})$, diluted with ethyl acetate $(2.0 \mathrm{~mL})$, and allowed to warm to $22{ }^{\circ} \mathrm{C}$. The two phases were separated, and the aqueous layer was extracted with ethyl acetate $(3 \times 5 \mathrm{~mL})$. The combined organic phases were washed with an aqueous solution of potassium hydroxide $(2.0 \mathrm{M}, 2 \times 5 \mathrm{~mL})$, dried over anhydrous sodium sulfate, and concentrated under reduced pressure. The obtained residue was purified by flash column chromatography (silica gel, $50 \rightarrow 10 \%$ hexanes in ethyl acetate) to afford vinyl iodide 25 (75.1 mg, $0.177 \mathrm{mmol}, 98 \%$ yield) as a colorless oil.

$\mathbf{R}_{f}=0.23$ (silica gel, 15\% hexanes in ethyl acetate, UV, $p$-anisaldehyde). $[\boldsymbol{\alpha}]_{\mathbf{D}}^{\mathbf{2 2}}=-78.7\left(c=0.60, \mathrm{CH}_{2} \mathrm{Cl}_{2}\right)$. 


\section{Rhoades, * et al.}

FT-IR (thin film): $v_{\max } 3421,3058,2956,2928,2872,1714,1670,1616,1541,1457,1435,1370,1340$, $1282,1259,1215,1170,1126,1101,1079,1060,1049,1020,978,943,904,827,784,735,701$, $675 \mathrm{~cm}^{-1}$.

${ }^{1} \mathbf{H}$ NMR (600 MHz, CDCl): $\delta 6.47(\mathrm{br} \mathrm{q}, J=0.8 \mathrm{~Hz}, 1 \mathrm{H}), 5.74(\mathrm{dd}, J=15.2,9.7 \mathrm{~Hz}, 1 \mathrm{H}), 5.39(\mathrm{dd}, J$ $=15.2,9.9 \mathrm{~Hz}, 1 \mathrm{H}), 5.29(\mathrm{~d}, J=10.7 \mathrm{~Hz}, 1 \mathrm{H}), 3.80(\mathrm{~d}, J=9.7 \mathrm{~Hz}, 1 \mathrm{H}), 3.78-3.72(\mathrm{~m}, 1 \mathrm{H}), 3.40(\mathrm{br} \mathrm{d}$, $J=10.0 \mathrm{~Hz}, 1 \mathrm{H}), 2.64(\mathrm{dd}, J=15.0,3.8 \mathrm{~Hz}, 1 \mathrm{H}), 2.54(\mathrm{dd}, J=15.0,3.0 \mathrm{~Hz}, 1 \mathrm{H}), 2.51(\mathrm{ddq}, J=10.7$, 9.9, $6.8 \mathrm{~Hz}, 1 \mathrm{H}), 2.37$ (br s, $1 \mathrm{H}), 1.86$ (br s, $1 \mathrm{H}), 1.83$ (d, $J=0.8 \mathrm{~Hz}, 3 \mathrm{H}), 1.69$ (dddd, $J=13.6,13.1$, 4.1, $4.0 \mathrm{~Hz}, 1 \mathrm{H}), 1.47$ (app td, $J=12.9,3.6 \mathrm{~Hz}, 1 \mathrm{H}), 1.40$ (app td, $J=12.9,4.4 \mathrm{~Hz}, 1 \mathrm{H}), 1.31$ (br s, 3 H), 1.26-1.19 (m, $1 \mathrm{H}), 0.92(\mathrm{~d}, J=6.8 \mathrm{~Hz}, 3 \mathrm{H}) \mathrm{ppm}$.

${ }^{13}$ C NMR (151 MHz, CDCl $): ~ \delta 172.1,143.5,136.7,130.8,84.3,80.4,77.2,73.5,69.4,41.0,38.4,35.7$, 29.9, 24.6, 19.2, $16.6 \mathrm{ppm}$.

HRMS (ESI, $\boldsymbol{m} / z)$ ): calcd for $\mathrm{C}_{16} \mathrm{H}_{25} \mathrm{IO}_{5} \mathrm{Na}^{+}[\mathrm{M}+\mathrm{Na}]^{+} 447.0639$, found 447.0649, $\Delta 2.2 \mathrm{ppm}$.
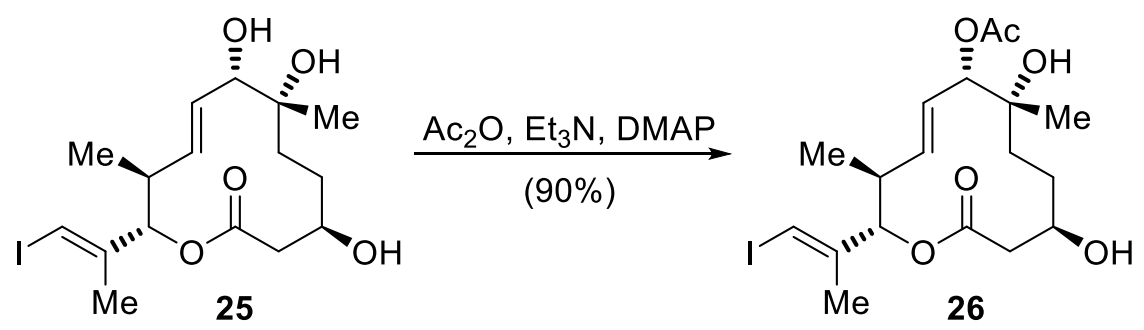

$(2 S, 3 S, 6 S, 7 R, 10 R, E)-7,10$-Dihydroxy-2-[(E)-1-iodoprop-1-en-2-yl]-3,7-dimethyl-12-

oxooxacyclododec-4-en-6-yl acetate (26): To a stirred solution of vinyl iodide 25 (54.0 mg, 0.127 mmol, 1.0 equiv) in dichloromethane $(1.3 \mathrm{~mL})$ at $0{ }^{\circ} \mathrm{C}$ was added triethylamine $(53 \mu \mathrm{L}, 0.381 \mathrm{mmol}, 3.0$ equiv) and 4-dimethylaminopyridine ( $3.1 \mathrm{mg} .25 \mu \mathrm{mol}, 0.2$ equiv), followed by the dropwise addition of a solution of acetic anhydride (1.0 M dichloromethane, $0.15 \mathrm{~mL}, 0.15 \mathrm{mmol}, 1.2$ equiv). After stirring for $30 \mathrm{~min}$, the reaction mixture was quenched with a saturated aqueous solution of ammonium chloride (3 $\mathrm{mL}$ ), and allowed to warm to $22{ }^{\circ} \mathrm{C}$. The two phases were separated, and the aqueous layer was extracted with ethyl acetate $(3 \times 5 \mathrm{~mL})$. The combined organic phases were dried over anhydrous sodium sulfate 


\section{Rhoades, * et al.}

and concentrated under reduced pressure. The obtained residue was purified by flash column chromatography (silica gel, $50 \rightarrow 15 \%$ hexanes in ethyl acetate) to afford acetyl ester 26 (53.0 mg, 0.114 mmol, $90 \%$ yield) as a colorless oil.

$\mathbf{R}_{f}=0.37$ (silica gel, 15\% hexanes in ethyl acetate, UV, $p$-anisaldehyde).

$[\boldsymbol{\alpha}]_{\mathbf{D}}^{22}=-41.7\left(c=0.30, \mathrm{CH}_{2} \mathrm{Cl}_{2}\right)$.

FT-IR (thin film): $v_{\max } 3502,3058,2954,2929,2870,2854,1735,1716,1674,1617,1541,1457,1436$, $1411,1369,1339,1281,1243,1169,1135,1103,1060,1020,980,943,913,864,819,786,745,707$, $674 \mathrm{~cm}^{-1}$.

${ }^{1}$ H NMR (600 MHz, CDCl $): \delta 6.48($ br q, $J=0.9 \mathrm{~Hz}, 1 \mathrm{H}), 5.68(\mathrm{dd}, J=15.2,9.7 \mathrm{~Hz}, 1 \mathrm{H}), 5.58(\mathrm{~d}, J=$ 15.2, 9.8 Hz, $1 \mathrm{H}), 5.30(\mathrm{~d}, J=10.7 \mathrm{~Hz}, 1 \mathrm{H}), 5.06$ (d, $J=9.7 \mathrm{~Hz}, 1 \mathrm{H}), 3.79-3.73(\mathrm{~m}, 1 \mathrm{H}), 3.42(\mathrm{~d}, J=$ $11.0 \mathrm{~Hz}, 1 \mathrm{H}), 2.64(\mathrm{dd}, J=15.1,3.7 \mathrm{~Hz}, 1 \mathrm{H}), 2.54(\mathrm{dd}, J=15.1,3.0 \mathrm{~Hz}, 1 \mathrm{H}), 2.49(\mathrm{ddq}, J=10.7,9.8$, $6.8 \mathrm{~Hz}, 1 \mathrm{H}), 2.09$ (br s, $1 \mathrm{H}), 2.09$ (s, $3 \mathrm{H}), 1.82$ (d, J = 0.9 Hz, $3 \mathrm{H}), 1.70$ (app tt, J = 13.5, 3.8 Hz, $1 \mathrm{H})$, $1.51(\operatorname{app} t d, J=14.2,3.4 \mathrm{~Hz}, 1 \mathrm{H}), 1.38(\operatorname{app} t d, J=14.2,4.2 \mathrm{~Hz}, 1 \mathrm{H}), 1.31-1.23(\mathrm{~m}, 1 \mathrm{H}), 1.21$ (br s, 3 H), $0.90(\mathrm{~d}, J=6.8 \mathrm{~Hz}, 3 \mathrm{H}) \mathrm{ppm}$.

${ }^{13}$ C NMR (151 MHz, CDCl3): $\delta$ 171.9, 169.8, 143.4, 139.8, 126.2, 84.4, 80.3, 78.8, 73.5, 69.2, 41.0, 38.4, $35.2,29.9,24.7,21.4,19.2,16.5 \mathrm{ppm}$.

HRMS (ESI, $\boldsymbol{m} / z$ ): calcd for $\mathrm{C}_{18} \mathrm{H}_{27} \mathrm{IO}_{6} \mathrm{Na}^{+}[\mathrm{M}+\mathrm{Na}]^{+} 489.0745$, found 489.0749, $\Delta 0.8 \mathrm{ppm}$.

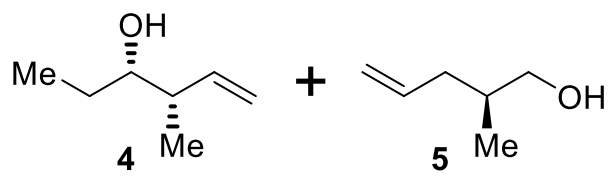

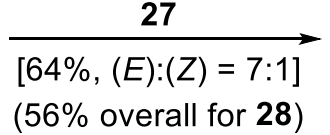

[gram scale]

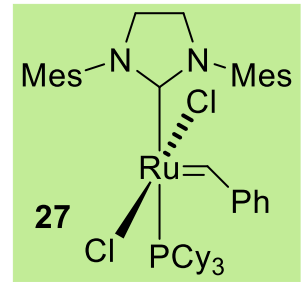

(2S,6S,7S,E)-2,6-Dimethylnon-4-ene-1,7-diol (28): To a stirred solution of terminal olefins 4 (1.52 g, $13.3 \mathrm{mmol}, 1.0$ equiv) and 5 (2.66 g, $26.6 \mathrm{mmol}, 2.0$ equiv) in dichloromethane $(67 \mathrm{~mL})$ at $50{ }^{\circ} \mathrm{C}$ was added a solution of Grubbs $2^{\text {nd }}$ Generation catalyst (27) in dichloromethane (1.0 M, $0.93 \mathrm{~mL}, 0.93 \mathrm{mmol}$, 


\section{Rhoades, * et al.}

0.07 equiv) dropwise via syringe pump over $30 \mathrm{~min}$. After stirring for an additional $5.5 \mathrm{~h}$, the reaction mixture was allowed to cool to $22{ }^{\circ} \mathrm{C}$ and concentrated under reduced pressure. The crude residue was purified by flash column chromatography $(10 \rightarrow 30 \rightarrow 50 \%$ ethyl acetate in hexanes) to afford diol 28 [1.59 g, $8.51 \mathrm{mmol}, 64 \%$ yield, $(E):(Z)=7: 1,56 \%$ overall yield for 28 ] as a colorless oil.

$\mathbf{R}_{f}=0.26$ (silica gel, 50\% ethyl acetate in hexanes, $p$-anisaldehyde).

$[\boldsymbol{\alpha}]_{\mathbf{D}}^{22}=-22.2\left(c=0.60, \mathrm{CH}_{2} \mathrm{Cl}_{2}\right)$.

FT-IR (thin film): $v_{\max } 3339,2960,2875,1458,1438,1376,1306,1275,1259,1131,1098,1040,970$, $909,857,764,749,674 \mathrm{~cm}^{-1}$.

${ }^{1}$ H NMR (600 MHz, CDCl 3$): \delta 5.50(\mathrm{dddd}, J=15.4,7.6,6.9,0.8 \mathrm{~Hz}, 1 \mathrm{H}), 5.41$ (dddd, $J=15.4,7.5$, 1.1, $0.9 \mathrm{~Hz}, 1 \mathrm{H}), 3.50$ (dd, $J=10.4,6.4 \mathrm{~Hz}, 1 \mathrm{H}), 3.46(\mathrm{dd}, J=10.4,5.8 \mathrm{~Hz}, 1 \mathrm{H}), 3.37$ (ddd, $J=8.6,4.8$, $3.8 \mathrm{~Hz}, 1 \mathrm{H}), 2.28-2.22(\mathrm{~m}, 1 \mathrm{H}), 2.14-2.09$ (m, $1 \mathrm{H}), 1.97-1.92(\mathrm{~m}, 1 \mathrm{H}), 1.74-1.69$ (m, $1 \mathrm{H}), 1.62$ (br s, $1 \mathrm{H}), 1.54(\mathrm{dqd}, J=14.5,7.4,3.8 \mathrm{~Hz}, 1 \mathrm{H}), 1.46($ br s, $1 \mathrm{H}), 1.36(\mathrm{ddq}, J=14.5,8.6,7.4 \mathrm{~Hz}, 1 \mathrm{H}), 1.00$ $(\mathrm{d}, J=6.9 \mathrm{~Hz}, 3 \mathrm{H}), 0.96(\operatorname{app~t}, J=7.4 \mathrm{~Hz}, 3 \mathrm{H}), 0.91(\mathrm{~d}, J=6.8 \mathrm{~Hz}, 3 \mathrm{H}) \mathrm{ppm}$.

${ }^{13}$ C NMR (151 MHz, CDCl3): $\delta$ 134.4, 129.3, 76.7, 68.2, 42.3, 37.1, 36.1, 27.0, 16.7, 14.9, 10.6 ppm. HRMS (ESI, $\boldsymbol{m} / z)$ ): calcd for $\mathrm{C}_{11} \mathrm{H}_{22} \mathrm{O}_{2} \mathrm{Na}^{+}[\mathrm{M}+\mathrm{Na}]^{+}$209.1512, found 209.1516, $\Delta 1.9 \mathrm{ppm}$.
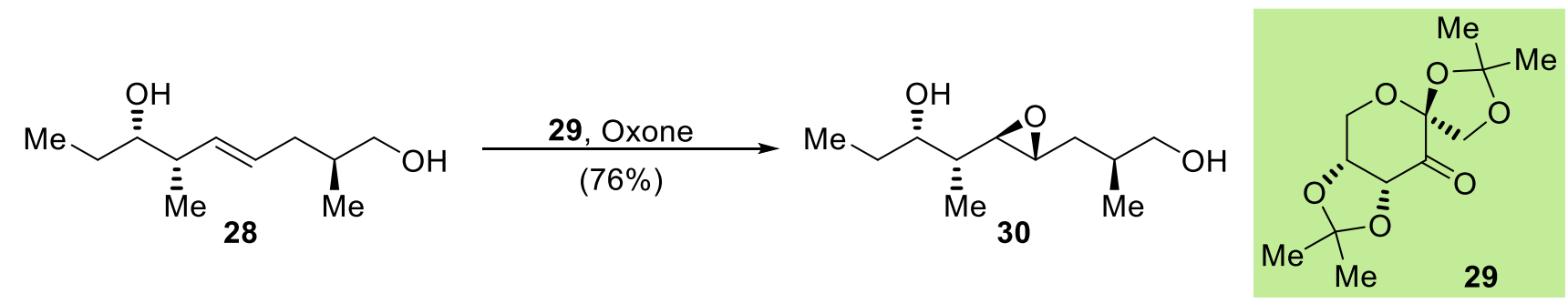

$(2 R, 3 S)-2-\{(2 R, 3 R)-3-[(S)-3-H y d r o x y-2-m e t h y l p r o p y l] o x i r a n-2-y l\}$ pentan-3-ol (30): To a vigorously stirred solution of diol 28 (512 mg, 2.75 mmol, 1.0 equiv) in a mixture of MeCN (40 mL) and pH 9.3 buffer (28 mL, 0.05 M sodium tetraborate decahydrate in $0.4 \mathrm{mM}$ aqueous $\mathrm{Na}_{2} \mathrm{EDTA}$ solution) at $22{ }^{\circ} \mathrm{C}$ was added 1,2:4,5-Di-O-isopropylidene- $\beta$-D-erythro-2,3-hexodiulo-2,6-pyranose (29) (2.49 g, 9.63 mmol, 3.5 equiv). After the biphasic reaction mixture became homogeneous (NOTE: this is critical for 


\section{Rhoades, * et al.}

complete conversion of starting material), it was cooled to $0{ }^{\circ} \mathrm{C}$. Next, a solution of potassium carbonate (4.66 g, $33.7 \mathrm{mmol}, 12.3$ equiv) in $0.4 \mathrm{mM}$ aqueous $\mathrm{Na}_{2}$ EDTA $(37 \mathrm{~mL})$ and a solution of potassium peroxymonosulfate (Oxone ${ }^{\circledR}, 3.47 \mathrm{~g}, 11.3 \mathrm{mmol}$, 4.1 equiv) in $0.4 \mathrm{mM}$ aqueous $\mathrm{Na}_{2} \mathrm{EDTA}(27 \mathrm{~mL}$ ) were added simultaneously dropwise via glass pipette sporadically every 15 minutes over the course of $1 \mathrm{~h}$ [NOTE: contact with trace metals (e.g., spatulas, needles, etc.) will decompose Oxone®]. After stirring for an additional $1 \mathrm{hr}$ at $0{ }^{\circ} \mathrm{C}$, the reaction mixture was diluted with ethyl acetate and allowed to warm to $22{ }^{\circ} \mathrm{C}$. The two phases were separated, and the aqueous layer was extracted with ethyl acetate $(3 \times 15$ $\mathrm{mL}$ ). The combined organic phases were dried over anhydrous sodium sulfate and concentrated under reduced pressure. The obtained residue was purified by flash column chromatography (silica gel, $40 \rightarrow 80 \%$ ethyl acetate in hexanes) to afford epoxy diol $\mathbf{3 0}$ (423 $\mathrm{mg}, 2.09 \mathrm{mmol}, 76 \%$ yield) as a colorless oil.

$\mathbf{R}_{f}=0.26$ (silica gel, 30\% hexanes in ethyl acetate, $p$-anisaldehyde).

$[\boldsymbol{\alpha}]_{\mathbf{D}}^{22}=+7.0\left(c=1.00, \mathrm{CH}_{2} \mathrm{Cl}_{2}\right)$.

FT-IR (thin film): $v_{\max } 3376,2963,2931,2877,1653,1559,1459,1425,1379,1338,1275,1247,1140$, $1099,1041,976,903,857,837,788,764,748,667 \mathrm{~cm}^{-1}$.

${ }^{1} \mathrm{H}$ NMR (600 MHz, CDCl$): ~ \delta 3.61(\mathrm{ddd}, J=6.6,6.6,3.2 \mathrm{~Hz}, 1 \mathrm{H}), 3.53(\mathrm{dd}, J=10.8,5.6 \mathrm{~Hz}, 1 \mathrm{H})$, $3.49(\mathrm{dd}, J=10.8,7.0 \mathrm{~Hz}, 1 \mathrm{H}), 2.88(\mathrm{ddd}, J=7.0,4.8,2.4 \mathrm{~Hz}, 1 \mathrm{H}), 2.73(\mathrm{dd}, J=7.0,2.4 \mathrm{~Hz}, 1 \mathrm{H}), 2.15$ (br s, 2 H), 1.91-1.85 (m, 1 H), 1.70 (ddd, $J=14.3,6.0,4.8 \mathrm{~Hz}, 1 \mathrm{H}), 1.53-1.44(\mathrm{~m}, 4 \mathrm{H}), 0.99$ (d, $J=6.9$ $\mathrm{Hz}, 3 \mathrm{H}), 0.95(\mathrm{~d}, J=7.1 \mathrm{~Hz}, 3 \mathrm{H}), 0.95(\operatorname{app~t}, J=7.4 \mathrm{~Hz}, 3 \mathrm{H}) \mathrm{ppm}$.

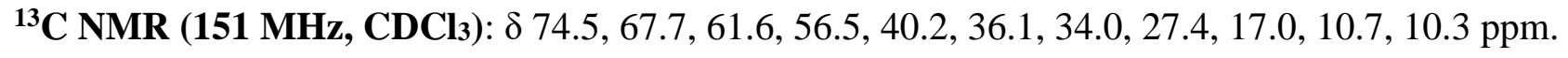
HRMS (ESI, $\boldsymbol{m} / z$ ): calcd for $\mathrm{C}_{11} \mathrm{H}_{22} \mathrm{O}_{3} \mathrm{Na}^{+}[\mathrm{M}+\mathrm{Na}]^{+} 225.1461$, found 225.1466, $\Delta 2.2 \mathrm{ppm}$. 


$$
\text { D. Rhoades,* et al. }
$$

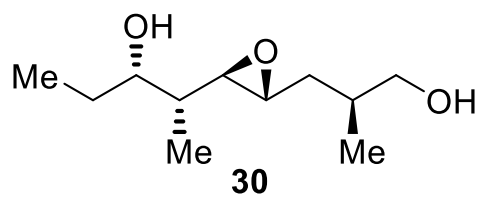

30

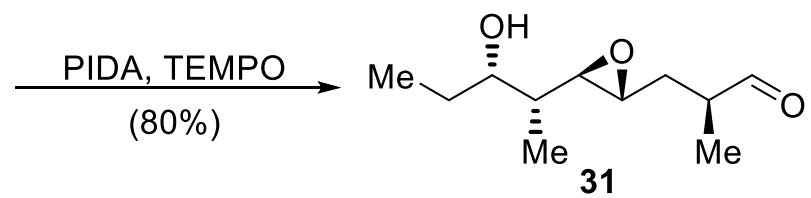

$(S)-3-\{(2 R, 3 R)-3-[(2 R, 3 S)-3-H y d r o x y p e n t a n-2-y l] o x i r a n-2-y l\}-2-m e t h y l p r o p a n a l ~(31):$ To a stirred solution of epoxy diol 28 (357 mg, $1.76 \mathrm{mmol}, 1.0$ equiv) in dichloromethane $(3.5 \mathrm{~mL})$ at $22{ }^{\circ} \mathrm{C}$ was added phenyliodine(III) acetate (625 mg, $1.94 \mathrm{mmol}, 1.1$ equiv) followed by (2,2,6,6tetramethylpiperidin-1-yl)oxyl (27.5 mg, $0.176 \mathrm{mmol}, 0.1$ equiv). After stirring for $1 \mathrm{~h}$, the reaction mixture was quenched with a saturated aqueous solution of sodium thiosulfate $(1.5 \mathrm{~mL})$ and a saturated aqueous solution of sodium bicarbonate $(1.5 \mathrm{~mL})$. The two phases were separated, and the aqueous layer was extracted with ethyl acetate $(3 \times 3 \mathrm{~mL})$. The combined organic phases were dried over anhydrous sodium sulfate and concentrated under reduced pressure. The obtained residue was purified by flash column chromatography (silica gel, $25 \rightarrow 55 \%$ ethyl acetate in hexanes) to afford epoxy aldehyde $\mathbf{3 1}$ (282 $\mathrm{mg}, 1.41 \mathrm{mmol}, 80 \%$ yield) as a colorless oil.

$\mathbf{R}_{f}=0.39$ (silica gel, 50\% ethyl acetate hexanes, $p$-anisaldehyde).

$[\boldsymbol{\alpha}]_{\mathbf{D}}^{22}=+17.5\left(c=1.00, \mathrm{CH}_{2} \mathrm{Cl}_{2}\right)$.

FT-IR (thin film): $v_{\max } 3443,2967,2934,2878,2723,1723,1558,1458,1397,1377,1333,1245,1140$, $1100,1056,1042,1024,975,965,907,841,816,796,754,667 \mathrm{~cm}^{-1}$.

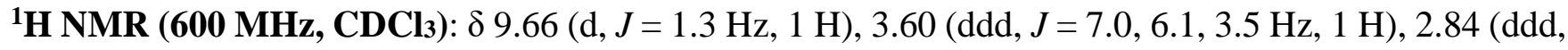
$J=6.9,4.8,2.3 \mathrm{~Hz}, 1 \mathrm{H}), 2.73(\mathrm{dd}, J=7.3,2.3 \mathrm{~Hz}, 1 \mathrm{H}), 2.58(\mathrm{dqdd}, J=7.6,7.2,6.1,1.3 \mathrm{~Hz}, 1 \mathrm{H}), 2.02$ (ddd, $J=14.3,7.6,4.8 \mathrm{~Hz}, 1 \mathrm{H}), 1.86(\mathrm{br} \mathrm{s}, 1 \mathrm{H}), 1.55(\mathrm{ddd}, J=14.3,6.9,6.1 \mathrm{~Hz}, 1 \mathrm{H}), 1.53-1.47(\mathrm{~m}, 2$ H), $1.45(\mathrm{dqd}, J=7.3,7.0,3.5 \mathrm{~Hz}, 1 \mathrm{H}), 1.21(\mathrm{~d}, J=7.2 \mathrm{~Hz}, 3 \mathrm{H}), 0.95$ (d, $J=7.0 \mathrm{~Hz}, 3 \mathrm{H}), 0.95$ (app t, $J=7.4 \mathrm{~Hz}, 3 \mathrm{H}) \mathrm{ppm}$.

${ }^{13}$ C NMR (151 MHz, CDCl3): $\delta$ 204.0, 74.5, 61.6, 55.9, 44.3, 40.2, 33.2, 27.4, 14.1, 10.6, 10.5 ppm. HRMS (CI, $\boldsymbol{m} / z)$ : calcd for $\mathrm{C}_{11} \mathrm{H}_{20} \mathrm{O}_{3}{ }^{++}[\mathrm{M} \cdot]^{+} 200.1407$, found 200.1401, $\Delta 3.0 \mathrm{ppm}$. 


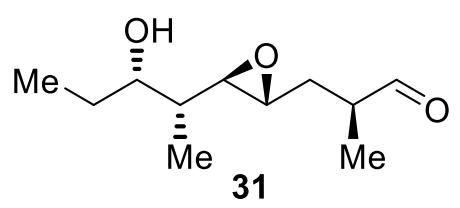

D. Rhoades, * et al.

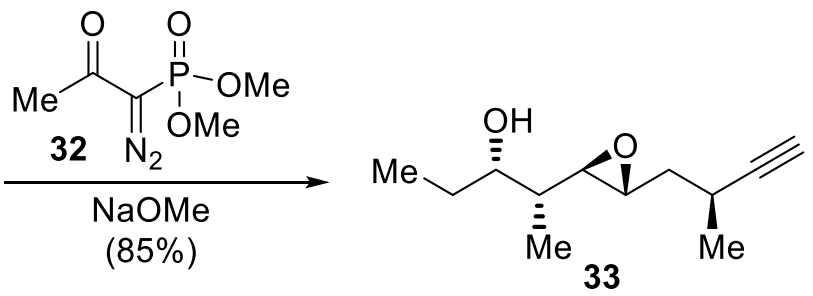

$(2 R, 3 S)-2-\{(2 R, 3 R)-3-[(S)-2-M e t h y l b u t-3-y n-1-y l] o x i r a n-2-y l\} p e n t a n-3-o l ~(33):$ To a stirred solution of dimethyl (1-diazo-2-oxopropyl)phosphonate 32 (1.02 g, $5.30 \mathrm{mmol}, 5.0$ equiv) in THF (5.3 mL) at $-78{ }^{\circ} \mathrm{C}$ was added a freshly prepared solution of sodium methoxide* $(0.5 \mathrm{M} \mathrm{THF}, 10.6 \mathrm{~mL}, 5.30 \mathrm{mmol}$, 5.0 equiv), and the bright yellow suspension was stirred for an additional $15 \mathrm{~min}$. Then a solution of epoxy aldehyde 31 (212 mg, $1.06 \mathrm{mmol}, 1.0$ equiv) in THF (2.1 mL) was added dropwise via cannula carefully down the side of the reaction vessel, the original flask was rinsed with additional tetrahydrofuran $(3 \times 0.5$ $\mathrm{mL}$ ), and the reaction mixture was stirred for an additional 15 min before being allowed to slowly warm to $-50^{\circ} \mathrm{C}$ over a period of $1.5 \mathrm{~h}$. Then the reaction mixture was quenched with a saturated aqueous solution of ammonium chloride ( $5 \mathrm{~mL})$, and allowed to warm to $22{ }^{\circ} \mathrm{C}$. The two phases were separated, and the aqueous layer was extracted with ethyl acetate $(3 \times 10 \mathrm{~mL})$. The combined organic phases were dried over anhydrous sodium sulfate and concentrated under reduced pressure. The obtained residue was purified by flash column chromatography (silica gel, $25 \rightarrow 50 \%$ diethyl ether in hexanes) to afford alkyne 33 (177 mg, $0.901 \mathrm{mmol}, 85 \%$ yield) as a colorless oil. *NOTE: A solution of sodium methoxide was prepared via the dropwise addition of anhydrous methanol to a stirred, equimolar suspension of $\mathrm{NaH}(60 \%$ $w / w$ in mineral oil) in tetrahydrofuran (ca. $0.5 \mathrm{M}$ ) at $22^{\circ} \mathrm{C}$, followed by stirring for an additional $30 \mathrm{~min}$. $\mathbf{R}_{f}=0.28$ (silica gel, 50\% diethyl ether in hexanes, $p$-anisaldehyde).

$[\boldsymbol{\alpha}]_{\mathbf{D}}^{22}=+47.2\left(c=1.00, \mathrm{CH}_{2} \mathrm{Cl}_{2}\right)$.

FT-IR (thin film): $v_{\max } 3445,3309,2970,2936,2877,2114,1638,1455,1377,1330,1244,1141,1110$, $1068,1039,1021,964,908,883,849,778,750 \mathrm{~cm}^{-1}$.

${ }^{1}$ H NMR (600 MHz, CDCl3): $\delta 3.63(\operatorname{app} t d, J=6.5,3.5 \mathrm{~Hz}, 1 \mathrm{H}), 2.97(\mathrm{ddd}, J=6.3,5.5,2.2 \mathrm{~Hz}, 1 \mathrm{H})$, $2.79(\mathrm{dd}, J=7.2,2.2 \mathrm{~Hz}, 1 \mathrm{H}), 2.70-2.64(\mathrm{~m}, 1 \mathrm{H}), 2.11(\mathrm{~d}, J=2.4 \mathrm{~Hz}, 1 \mathrm{H}), 1.84$ (br s, $1 \mathrm{H}), 1.70-1.62$ 


\section{Rhoades, * et al.}

$(\mathrm{m}, 2 \mathrm{H}), 1.55-1.49(\mathrm{~m}, 3 \mathrm{H}), 1.25(\mathrm{~d}, J=7.0 \mathrm{~Hz}, 3 \mathrm{H}), 1.00(\mathrm{~d}, J=7.1 \mathrm{~Hz}, 3 \mathrm{H}), 0.97(\operatorname{app~t}, J=7.4 \mathrm{~Hz}$, $3 \mathrm{H}) \mathrm{ppm}$.

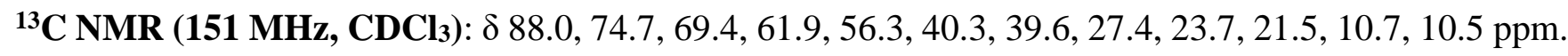
HRMS (ESI, $\boldsymbol{m} / \boldsymbol{z}$ ): calcd for $\mathrm{C}_{12} \mathrm{H}_{20} \mathrm{O}_{2} \mathrm{Na}^{+}[\mathrm{M}+\mathrm{Na}]^{+} 219.1356$, found 219.1359, $\Delta 1.4$ ppm.
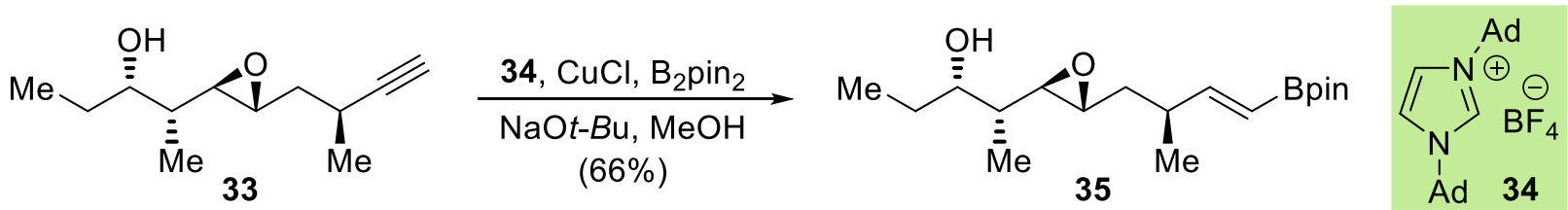

$(2 R, 3 S)-2-\{(2 R, 3 R)-3-[(S, E)-2-M e t h y l-4-(4,4,5,5-t e t r a m e t h y l-1,3,2-d i o x a b o r o l a n-2-y l) b u t-3-e n-1-$

yl]oxiran-2-yl\}pentan-3-ol (35): To a stirred suspension of copper(I) chloride (8.5 mg, $86 \mu \mathrm{mol}, 0.15$ equiv) and 1,3-bis(1-adamantyl)imidazolium tetrafluoroborate 34 (36.4 mg, $85.7 \mu \mathrm{mol}, 0.15$ equiv) in tetrahydrofuran $(2.4 \mathrm{~mL})$ at $22{ }^{\circ} \mathrm{C}$ was added sodium tert-butoxide (33.0 $\mathrm{mg}, 0.343 \mathrm{mmol}, 0.6$ equiv). After $1 \mathrm{~h}$, bis(pinacolato)diboron (174 mg, $0.685 \mathrm{mmol}, 1.2$ equiv) was added, and stirring was continued for an additional $30 \mathrm{~min}$. Then a solution of alkyne $\mathbf{3 3}(112 \mathrm{mg}, 0.571 \mathrm{mmol}, 1.0$ equiv) in tetrahydrofuran (0.5 mL) was added, followed by anhydrous methanol (0.50 mL, $1.2 \mathrm{mmol}, 2.1$ equiv). After $12 \mathrm{~h}$, the reaction mixture was quenched with a saturated aqueous solution of ammonium chloride (5 $\mathrm{mL})$, and the two phases were separated. The aqueous layer was extracted with ethyl acetate $(3 \times 3 \mathrm{~mL})$, and the combined organic phases were dried over anhydrous sodium sulfate and concentrated under reduced pressure. The obtained residue was purified by flash column chromatography (silica gel, $5 \rightarrow 20 \%$ diethyl ether in hexanes) to afford vinyl boronate ester 35 (122 mg, $0.377 \mathrm{mmol}, 66 \%$ yield) as a colorless oil. $\mathbf{R}_{f}=0.26$ (silica gel, 50\% diethyl ether in hexanes, $p$-anisaldehyde).

$[\boldsymbol{\alpha}]_{\mathbf{D}}^{22}=+27.8\left(c=1.00, \mathrm{CH}_{2} \mathrm{Cl}_{2}\right)$.

FT-IR (thin film): $v_{\max } 3470,2975,2929,2876,1637,1457,1397,1363,1326,1272,1233,1214,1165$, $1145,1107,999,970,924,901,849,657 \mathrm{~cm}^{-1}$. 


\section{Rhoades, * et al.}

${ }^{1}$ H NMR (600 MHz, CDCl 3$): \delta 6.54(\mathrm{dd}, J=18.0,7.3 \mathrm{~Hz}, 1 \mathrm{H}), 5.45(\mathrm{dd}, J=18.0,0.6 \mathrm{~Hz}, 1 \mathrm{H}), 3.58$ (app td, $J=6.6,3.6 \mathrm{~Hz}, 1 \mathrm{H}), 2.77(\operatorname{app} \mathrm{td}, J=5.9,2.2 \mathrm{~Hz}, 1 \mathrm{H}), 2.66(\mathrm{dd}, J=7.3,2.2 \mathrm{~Hz}, 1 \mathrm{H}), 2.49-$ $2.42(\mathrm{~m}, 1 \mathrm{H}), 1.94$ (br s, $1 \mathrm{H}), 1.62$ (app dt, $J=13.9,5.8 \mathrm{~Hz}, 1 \mathrm{H}), 1.53-1.47$ (m, $3 \mathrm{H}), 1.46-1.41(\mathrm{~m}, 1$ H), 1.26 (br s, $12 \mathrm{H}), 1.07$ (d, $J=6.8 \mathrm{~Hz}, 3 \mathrm{H}), 0.95(\operatorname{app~t}, J=7.4 \mathrm{~Hz}, 3 \mathrm{H}), 0.95(\mathrm{~d}, J=7.0 \mathrm{~Hz}, 3 \mathrm{H})$ ppm.

${ }^{13}$ C NMR (151 MHz, CDCl3): $\delta 158.3,117.7, * 83.3,74.7,62.1,56.9,40.3,38.9,37.8,27.3,24.9,20.3$, 10.71, 10.68 ppm. *Extensive line broadening due to $\mathrm{T}_{2}$ scalar coupling caused by the ${ }^{11} \mathrm{~B}$ quadrupole. ${ }^{22}$ HRMS (ESI, $\boldsymbol{m} / \boldsymbol{z}$ ): calcd for $\mathrm{C}_{18} \mathrm{H}_{33} \mathrm{BO}_{4} \mathrm{Na}^{+}[\mathrm{M}+\mathrm{Na}]^{+} 347.2364$, found 347.2374, $\Delta 2.9 \mathrm{ppm}$.

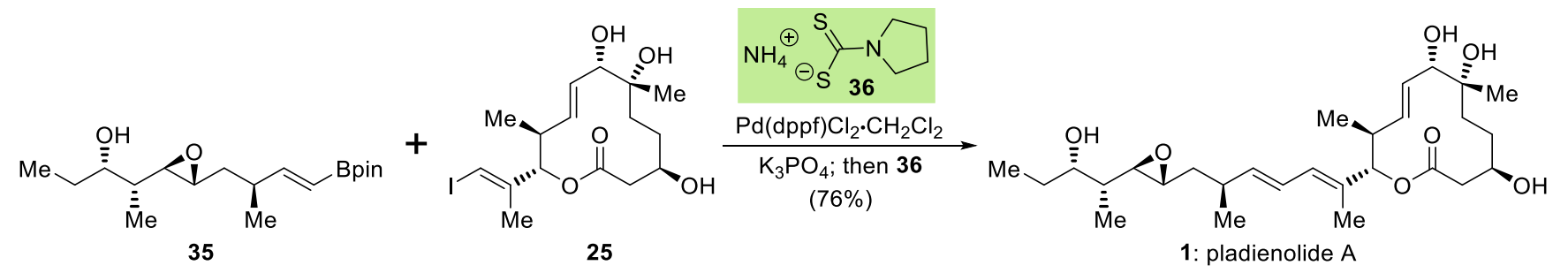

$(4 R, 7 R, 8 S, 11 S, 12 S, E)-4,7,8-T r i h y d r o x y-12-\{(S, 2 E, 4 E)-7-[(2 R, 3 R)-3-\{(2 R, 3 S)-3-h y d r o x y p e n t a n-2-$ yl\}oxiran-2-yl]-6-methylhepta-2,4-dien-2-yl\}-7,11-dimethyloxacyclododec-9-en-2-one (pladienolide A; 1): To a stirred solution of vinyl iodide $25(25.0 \mathrm{mg}, 58.9 \mu \mathrm{mol}, 1.0$ equiv) and vinyl boronate 35 (23.0 $\mathrm{mg}, 70.9 \mu \mathrm{mol}, 1.2$ equiv) in degassed (sparged with argon for $15 \mathrm{~min})$ aqueous tetrahydrofuran $(0.75$ mL, THF: $\left.\mathrm{H}_{2} \mathrm{O}=3: 1\right)$ at $22{ }^{\circ} \mathrm{C}$ was added $\mathrm{Pd}(\mathrm{dppf}) \mathrm{Cl}_{2} \cdot \mathrm{CH}_{2} \mathrm{Cl}_{2}(4.8 \mathrm{mg}, 5.9 \mu \mathrm{mol}, 0.1$ equiv) followed by potassium phosphate (38 mg, $0.18 \mathrm{mmol}, 3.0$ equiv). After stirring for $3 \mathrm{~h}$, ammonium pyrrolidinedithiocarbamate (36) (4.9 mg, $0.030 \mathrm{mmol}, 0.5$ equiv) was added, and stirring was continued for $1 \mathrm{~h}$. Then the reaction mixture was filtered through a layer of celite, rinsed thoroughly with ethyl acetate $(20 \mathrm{~mL})$, and concentrated under reduced pressure. The obtained residue was purified by flash column chromatography ( silica gel, $20 \%$ hexanes in ethyl acetate $\rightarrow 100 \%$ ethyl acetate) to afford pure pladienolide A (1) (22.0 mg, $44.5 \mu \mathrm{mol}, 76 \%$ yield) as a colorless oil.

$\mathbf{R}_{f}=0.38$ (silica gel, ethyl acetate, UV, $p$-anisaldehyde).

$[\boldsymbol{\alpha}]_{\mathrm{D}}^{22}=-1.5(c=1.00, \mathrm{MeOH})$ 


\section{Rhoades, * et al.}

FT-IR (thin film): $v_{\max } 3403,3027,2961,2928,2874,2854,1702,1667,1557,1456,1428,1408,1371$, $1340,1285,1249,1215,1175,1128,1101,1079,1050,1020,975,961,927,903,877,828,789,759$, $713,666 \mathrm{~cm}^{-1}$.

${ }^{1}$ H NMR (600 MHz, CD3OD): $\delta 6.37$ (dd, $\left.J=15.0,10.8 \mathrm{~Hz}, 1 \mathrm{H}\right), 6.12(\mathrm{dd}, J=10.8,0.8 \mathrm{~Hz}, 1 \mathrm{H}), 5.76$ $(\mathrm{dd}, J=15.0,9.8 \mathrm{~Hz}, 1 \mathrm{H}), 5.69(\mathrm{dd}, J=15.0,8.4 \mathrm{~Hz}, 1 \mathrm{H}), 5.42(\mathrm{dd}, J=15.0,9.8 \mathrm{~Hz}, 1 \mathrm{H}), 5.07(\mathrm{~d}, J=$ $10.8 \mathrm{~Hz}, 1 \mathrm{H}), 3.82-3.78(\mathrm{~m}, 1 \mathrm{H}), 3.74(\mathrm{~d}, J=9.8 \mathrm{~Hz}, 1 \mathrm{H}), 3.56(\mathrm{ddd}, J=8.7,4.5,4.5 \mathrm{~Hz}, 1 \mathrm{H}), 2.77$ $(\mathrm{ddd}, J=5.9,2.2,2.2 \mathrm{~Hz}, 1 \mathrm{H}), 2.70(\mathrm{dd}, J=8.2,2.2 \mathrm{~Hz}, 1 \mathrm{H}), 2.60(\mathrm{ddq}, J=10.8,9.8,6.8 \mathrm{~Hz}, 1 \mathrm{H})$, 2.57-2.53 (m, $2 \mathrm{H}), 2.53-2.48(\mathrm{~m}, 1 \mathrm{H}), 1.79(\mathrm{~d}, J=0.8 \mathrm{~Hz}, 3 \mathrm{H}), 1.68(\mathrm{ddd}, J=13.8,5.6,5.5 \mathrm{~Hz}, 1 \mathrm{H})$, 1.64-1.59 (m, $1 \mathrm{H}), 1.59-1.54(\mathrm{~m}, 1 \mathrm{H}), 1.56-1.52(\mathrm{~m}, 2 \mathrm{H}), 1.52-1.48(\mathrm{~m}, 1 \mathrm{H}), 1.43-1.35$ (m, $2 \mathrm{H})$, 1.31 (br s, $3 \mathrm{H}), 1.24(\mathrm{dqd}, J=8.2,7.0,2.2 \mathrm{~Hz}, 1 \mathrm{H}), 1.12(\mathrm{~d}, J=6.8 \mathrm{~Hz}, 3 \mathrm{H}), 0.98(\operatorname{app~t}, J=7.4 \mathrm{~Hz}, 3$ $\mathrm{H}), 0.948(\mathrm{~d}, J=6.8 \mathrm{~Hz}, 3 \mathrm{H}), 0.945(\mathrm{~d}, J=7.0 \mathrm{~Hz}, 3 \mathrm{H}) \mathrm{ppm}$. *Due to solvent exchange with $\mathrm{CD}_{3} \mathrm{OD}$, $4-\mathrm{OH}$ protons were not observed.

${ }^{13}$ C NMR (151 MHz, CD3OD): $\delta$ 171.9, 142.3, 137.7, 132.6, 132.1, 131.6, 125.9, 84.4, 78.2, 75.3, 74.7, 70.7, 63.0, 58.5, 42.8, 41.8, 40.7, 40.1, 37.6, 36.8, 30.5, 28.6, 24.4, 21.7, 17.1, 11.9, 10.88, 10.86 ppm.

HRMS (ESI, $\boldsymbol{m} / z$ ): calcd for $\mathrm{C}_{28} \mathrm{H}_{46} \mathrm{O}_{7} \mathrm{Na}^{+}[\mathrm{M}+\mathrm{Na}]^{+}$517.3136, found 517.3146, $\Delta 1.9 \mathrm{ppm}$.

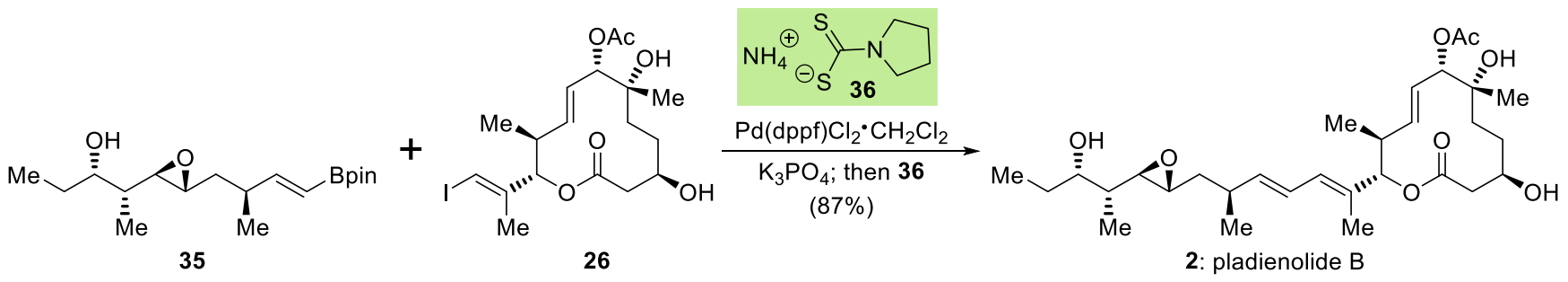

$(2 S, 3 S, 6 S, 7 R, 10 R, E)-7,10-D i h y d r o x y-2-\{(S, 2 E, 4 E)-7-[(2 R, 3 R)-3-\{(2 R, 3 S)-3-h y d r o x y p e n t a n-2-$

yl\}oxiran-2-yl]-6-methylhepta-2,4-dien-2-yl\}-3,7-dimethyl-12-oxooxacyclododec-4-en-6-yl acetate (pladienolide B; 2): To a stirred solution of vinyl iodide 26 (42.0 mg, $90.1 \mu$ mol, 1.0 equiv) and vinyl boronate 35 (35.0 mg, $108 \mu \mathrm{mol}, 1.2$ equiv) in degassed (sparged with argon for $15 \mathrm{~min}$ ) aqueous tetrahydrofuran $\left(1.1 \mathrm{~mL}, \mathrm{THF}: \mathrm{H}_{2} \mathrm{O}=3: 1\right)$ at $22{ }^{\circ} \mathrm{C}$ was added $\mathrm{Pd}(\mathrm{dppf}) \mathrm{Cl}_{2} \cdot \mathrm{CH}_{2} \mathrm{Cl}_{2}(7.3 \mathrm{mg}, 9.0 \mu \mathrm{mol}$, 


\section{Rhoades, * et al.}

0.1 equiv) followed by potassium phosphate $(57 \mathrm{mg}, 0.27 \mathrm{mmol}, 3.0$ equiv). After stirring for $3 \mathrm{~h}$, ammonium pyrrolidinedithiocarbamate (36) $(7.4 \mathrm{mg}, 45 \mu \mathrm{mol}, 0.5$ equiv) was added, and stirring was continued for $1 \mathrm{~h}$. Then the reaction mixture was filtered through a layer of celite, rinsed thoroughly with ethyl acetate $(20 \mathrm{~mL})$, and concentrated under reduced pressure. The obtained residue was purified by flash column chromatography (silica gel, $20 \%$ hexanes in ethyl acetate $\rightarrow 100 \%$ ethyl acetate) to afford pure pladienolide B (2) (42.0 mg, $78.3 \mu \mathrm{mol}, 87 \%$ yield) as a colorless oil.

$\mathbf{R}_{f}=0.50$ (silica gel, ethyl acetate, UV, $p$-anisaldehyde).

$[\boldsymbol{\alpha}]_{\mathbf{D}}^{\mathbf{2 2}}=+7.9(c=1.10, \mathrm{MeOH})$.

FT-IR (thin film): $v_{\max } 3424,2962,2929,2875,1730,1719,1684,1650,1559,1415,1373,1343,1243$, $1177,1100,1049,1021,978,928,911,878,823,791,780,703,671,660 \mathrm{~cm}^{-1}$.

${ }^{1}$ H NMR (600 MHz, CD3OD): $\delta 6.37$ (dd, $\left.J=15.0,10.8 \mathrm{~Hz}, 1 \mathrm{H}\right), 6.13(\mathrm{dd}, J=10.8,0.8 \mathrm{~Hz}, 1 \mathrm{H}), 5.74$ $(\mathrm{dd}, J=15.2,9.8 \mathrm{~Hz}, 1 \mathrm{H}), 5.70(\mathrm{dd}, J=15.0,8.5 \mathrm{~Hz}, 1 \mathrm{H}), 5.61(\mathrm{dd}, J=15.2,9.9 \mathrm{~Hz}, 1 \mathrm{H}), 5.09(\mathrm{~d}, J=$ $9.8 \mathrm{~Hz}, 1 \mathrm{H}), 5.09$ (d, $J=10.8 \mathrm{~Hz}, 1 \mathrm{H}), 3.84-3.80(\mathrm{~m}, 1 \mathrm{H}), 3.55$ (ddd, $J=8.7,4.5,4.5 \mathrm{~Hz}, 1 \mathrm{H}), 2.76$ (ddd, $J=6.0,5.5,2.2 \mathrm{~Hz}, 1 \mathrm{H}), 2.70(\mathrm{dd}, J=8.2,2.2 \mathrm{~Hz}, 1 \mathrm{H}), 2.61(\mathrm{ddq}, J=10.8,9.9,6.8 \mathrm{~Hz}, 1 \mathrm{H})$, 2.57-2.56 (m, $2 \mathrm{H}), 2.54-2.49$ (m, $1 \mathrm{H}), 2.10$ (s, $3 \mathrm{H}), 1.79(\mathrm{~d}, J=0.8 \mathrm{~Hz}, 3 \mathrm{H}), 1.70-1.66$ (m, $1 \mathrm{H}), 1.67-$ $1.64(\mathrm{~m}, 1 \mathrm{H}), 1.64-1.58(\mathrm{~m}, 1 \mathrm{H}), 1.58-1.51(\mathrm{~m}, 2 \mathrm{H}), 1.51-1.47$ (m, $1 \mathrm{H}), 1.46-1.41(\mathrm{~m}, 1 \mathrm{H}), 1.41-$ $1.37(\mathrm{~m}, 1 \mathrm{H}), 1.26-1.22(\mathrm{~m}, 1 \mathrm{H}), 1.23$ (br s, $3 \mathrm{H}), 1.12(\mathrm{~d}, J=6.8 \mathrm{~Hz}, 3 \mathrm{H}), 0.98(\operatorname{app~t}, J=7.4 \mathrm{~Hz}, 3$ H), $0.94(\mathrm{~d}, J=7.0 \mathrm{~Hz}, 3 \mathrm{H}), 0.92(\mathrm{~d}, J=6.8 \mathrm{~Hz}, 3 \mathrm{H}) \mathrm{ppm}$. *Due to solvent exchange with $\mathrm{CD}_{3} \mathrm{OD}$, $3-\mathrm{OH}$ protons were not observed.

${ }^{13}$ C NMR (151 MHz, CD3OD): $\delta$ 172.2, 171.7, 142.4, 141.7, 132.4, 132.2, 127.1, 125.9, 84.3, 80.3, 75.3, 74.1, 70.4, 63.0, 58.5, 42.8, 41.7, 40.7, 40.1, 37.5, 36.8, 30.4, 28.6, 24.2, 21.7, 21.1, 16.9, 11.9, 10.88, $10.86 \mathrm{ppm}$.

HRMS (ESI, $\boldsymbol{m} / \boldsymbol{z}$ ): calcd for $\mathrm{C}_{30} \mathrm{H}_{48} \mathrm{O}_{8} \mathrm{Na}^{+}[\mathrm{M}+\mathrm{Na}]^{+}$559.3241, found 559.3251, $\Delta 1.8$ ppm. 
D. Rhoades, ${ }^{*}$ et al.

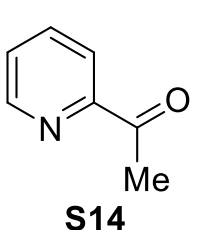

S14

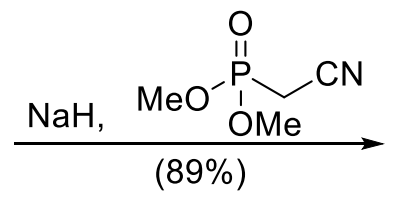

[Ref. 23]

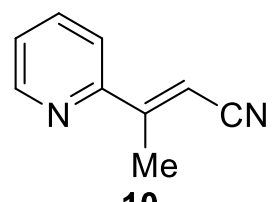

10

Scheme S11. Synthesis of nitrile 10 from commercially available 2-acetylpyridine (S14).

(E)-3-(Pyridin-2-yl)but-2-enenitrile (10): Nitrile 10 was prepared from commercially available 2acetylpyridine (S14) according to a literature procedure (Scheme S11). ${ }^{23}$ To a stirred solution of diethyl cyanomethylphosphonate $(8.67 \mathrm{~g}, 48.9 \mathrm{mmol}, 1.2 \mathrm{equiv})$ in tetrahydrofuran $(270 \mathrm{~mL})$ at $0{ }^{\circ} \mathrm{C}$ was carefully added sodium hydride (60\% w/w in mineral oil, $1.96 \mathrm{~g}, 48.9 \mathrm{mmol}, 1.2$ equiv) in portions. After stirring for $15 \mathrm{~min}$ at the same temperature, 2-acetylpyridine (S14) (4.58 mL, $40.8 \mathrm{mmol}, 1.0$ equiv) was added dropwise, and the reaction mixture slowly formed a yellow gummy precipitate. After an additional 30 min of vigorous stirring, the reaction mixture was quenched with a saturated aqueous solution of ammonium chloride $(50 \mathrm{~mL})$ and allowed to warm to $22{ }^{\circ} \mathrm{C}$. The two phases were separated, and the aqueous layer was extracted with ethyl acetate $(3 \times 15 \mathrm{~mL})$. The combined organic layers were dried over anhydrous sodium sulfate and concentrated under reduced pressure. The obtained residue was purified by flash column chromatography (silica gel, $5 \rightarrow 10 \rightarrow 25 \%$ ethyl acetate in hexanes) to afford nitrile $\mathbf{1 0}$ (5.23 g, $36.3 \mathrm{mmol}$, 89\% yield) as a colorless oil. The physical and spectral data are consistent with those previously reported. ${ }^{23}$

$\mathbf{R}_{f}=0.35$ (silica gel, 25\% ethyl acetate in hexanes, UV, potassium permanganate).

FT-IR (thin film): $v_{\max } 3059,3005,2985,2960,2926,2214,1613,1581,1568,1467,1432,1382,1330$, $1285,1272,1244,1225,1156,1102,1046,1015,992,966,926,893,852,772,741,659 \mathrm{~cm}^{-1}$.

${ }^{1}$ H NMR (600 MHz, CDCl3): $\delta 8.63(\mathrm{ddd}, J=4.7,1.8,0.8 \mathrm{~Hz}, 1 \mathrm{H}), 7.76(\mathrm{ddd}, J=7.9,7.6,1.8 \mathrm{~Hz}, 1$ H), $7.51(\mathrm{ddd}, J=7.9,1.0,0.8 \mathrm{~Hz}, 1 \mathrm{H}), 7.32(\mathrm{ddd}, J=7.6,4.7,1.0 \mathrm{~Hz}, 1 \mathrm{H}), 6.46(\mathrm{q}, J=1.1 \mathrm{~Hz}, 1 \mathrm{H})$, $2.50(\mathrm{~d}, J=1.1 \mathrm{~Hz}, 3 \mathrm{H}) \mathrm{ppm}$.

${ }^{13}$ C NMR (151 MHz, CDCl3): $\delta$ 157.1, 154.1, 149.7, 137.1, 124.7, 120.8, 117.9, 98.7, 18.5 ppm. 


\section{Rhoades, * et al.}

HRMS (ESI, $\boldsymbol{m} / z$ ): calcd for $\mathrm{C}_{9} \mathrm{H}_{9} \mathrm{~N}_{2}{ }^{+}[\mathrm{M}+\mathrm{H}]^{+} 145.0760$, found 145.0760 .
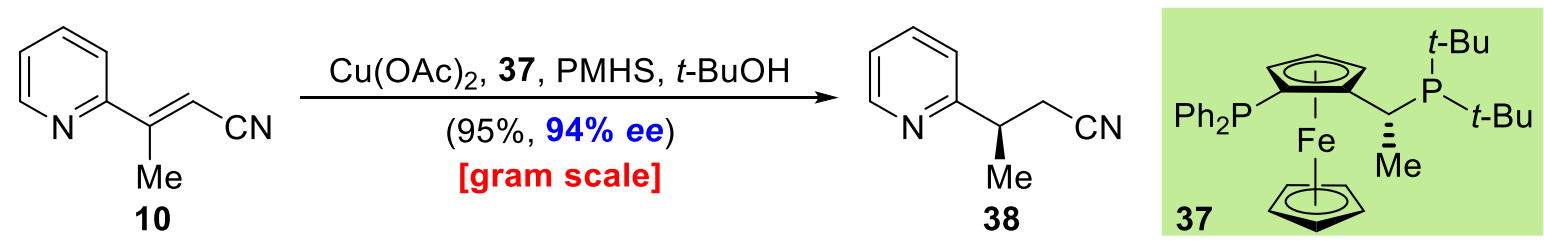

(R)-3-(Pyridin-2-yl)butanenitrile $\quad(\mathbf{3 8})$ : $\quad$ To a stirred solution of $\quad(2 R)-1-[(1 R)-1-[\operatorname{bis}(1,1-$ dimethylethyl)phosphino]ethyl]-2-(diphenylphosphino)ferrocene (37) (233 mg, $0.430 \mathrm{mmol}, 0.02$ equiv) and copper(II) acetate $\left(78.1 \mathrm{mg}, 0.430 \mathrm{mmol}, 0.02\right.$ equiv) in toluene $(21 \mathrm{~mL})$ at $0{ }^{\circ} \mathrm{C}$ was added polymethylhydrosiloxane $(5.18 \mathrm{~mL}, 86.0 \mathrm{mmol}, 4.0$ equiv). After $10 \mathrm{~min}$, the reaction mixture was cooled to $0{ }^{\circ} \mathrm{C}$, and a solution of nitrile $\mathbf{1 0}(3.10 \mathrm{~g}, 21.5 \mathrm{mmol}, 1.0$ equiv $)$ in toluene $(21 \mathrm{~mL})$ was added dropwise, followed by tert-butanol $(8.16 \mathrm{~mL}, 86.0 \mathrm{mmol}, 4.0$ equiv), and the reaction mixture was allowed to warm to $22{ }^{\circ} \mathrm{C}$ over $20 \mathrm{~min}$. After an additional 3.5 hours, the reaction mixture was diluted with diethyl ether (30 mL), quenched with an aqueous solution of sodium hydroxide $(2.5 \mathrm{M}, 50 \mathrm{~mL})$, and stirred vigorously for $30 \mathrm{~min}$. The two phases were separated, and the aqueous layer was extracted with ethyl acetate $(3 \times$ $20 \mathrm{~mL}$ ). The combined organic layers were dried over anhydrous sodium sulfate and concentrated under reduced pressure. The obtained residue was purified by flash column chromatography (silica gel, $10 \rightarrow 25 \% \rightarrow 35 \%$ ethyl acetate in hexanes) to afford nitrile $\mathbf{3 8}(2.98 \mathrm{~g}, 20.4 \mathrm{mmol}, 95 \%$ yield, $94 \%$ ee $)$ as a colorless oil. The enantiomeric excess was measured by normal phase chiral HPLC (CHIRALPAK®) AS-H, $5 \mu \mathrm{m}, \varphi 4.6 \times 250 \mathrm{~mm} ; 0 \rightarrow 1.5 \%$ isopropanol in hexanes; flow rate $=4.0 \mathrm{~mL} / \mathrm{min} ; 30 \mathrm{~min}$ run) with a racemic comparison prepared under the same conditions using Xantphos as an achiral ligand $(0.5 \mathrm{mmol}$ scale, $78 \%$ yield).

$\mathbf{R}_{f}=0.31$ (silica gel, 35\% ethyl acetate in hexanes).

$[\boldsymbol{\alpha}]_{\mathbf{D}}^{22}=-21.8\left(c=1.00, \mathrm{CH}_{2} \mathrm{Cl}_{2}\right)$.

FT-IR (thin film): $v_{\max } 3054,3011,2972,2932,2876,2247,1591,1571,1474,1435,1376,1328,1297$, $1271,1201,1150,1099,1072,1049,1024,992,946,893,853,786,750 \mathrm{~cm}^{-1}$. 


\section{Rhoades, * et al.}

${ }^{1} \mathbf{H}$ NMR (600 MHz, CDCl 3$): \delta 8.56(\mathrm{ddd}, J=4.8,1.8,0.8 \mathrm{~Hz}, 1 \mathrm{H}), 7.67(\mathrm{ddd}, J=7.8,7.6,1.8 \mathrm{~Hz}, 1$ H), $7.22(\mathrm{ddd}, J=7.8,1.0,0.8 \mathrm{~Hz}, 1 \mathrm{H}), 7.19(\mathrm{ddd}, J=7.6,4.8,1.0 \mathrm{~Hz}, 1 \mathrm{H}), 3.30(\mathrm{dqd}, J=7.3,7.0,6.7$ Hz, $1 \mathrm{H}), 2.84(\mathrm{dd}, J=16.7,6.7 \mathrm{~Hz}, 1 \mathrm{H}), 2.74(\mathrm{dd}, J=16.7,7.3 \mathrm{~Hz}, 1 \mathrm{H}), 1.46(\mathrm{~d}, J=7.0 \mathrm{~Hz}, 3 \mathrm{H}) \mathrm{ppm}$. ${ }^{13}$ C NMR (151 MHz, CDCl3): $\delta$ 161.7, 149.6, 137.1, 122.4, 122.0, 119.1, 38.4, 24.1, 20.3 ppm.

HRMS (ESI, $\boldsymbol{m} / \boldsymbol{z}$ ): calcd for $\mathrm{C}_{9} \mathrm{H}_{11} \mathrm{~N}_{2}{ }^{+}[\mathrm{M}+\mathrm{H}]^{+}$147.0917, found 147.0914, $\Delta 2.0 \mathrm{ppm}$.

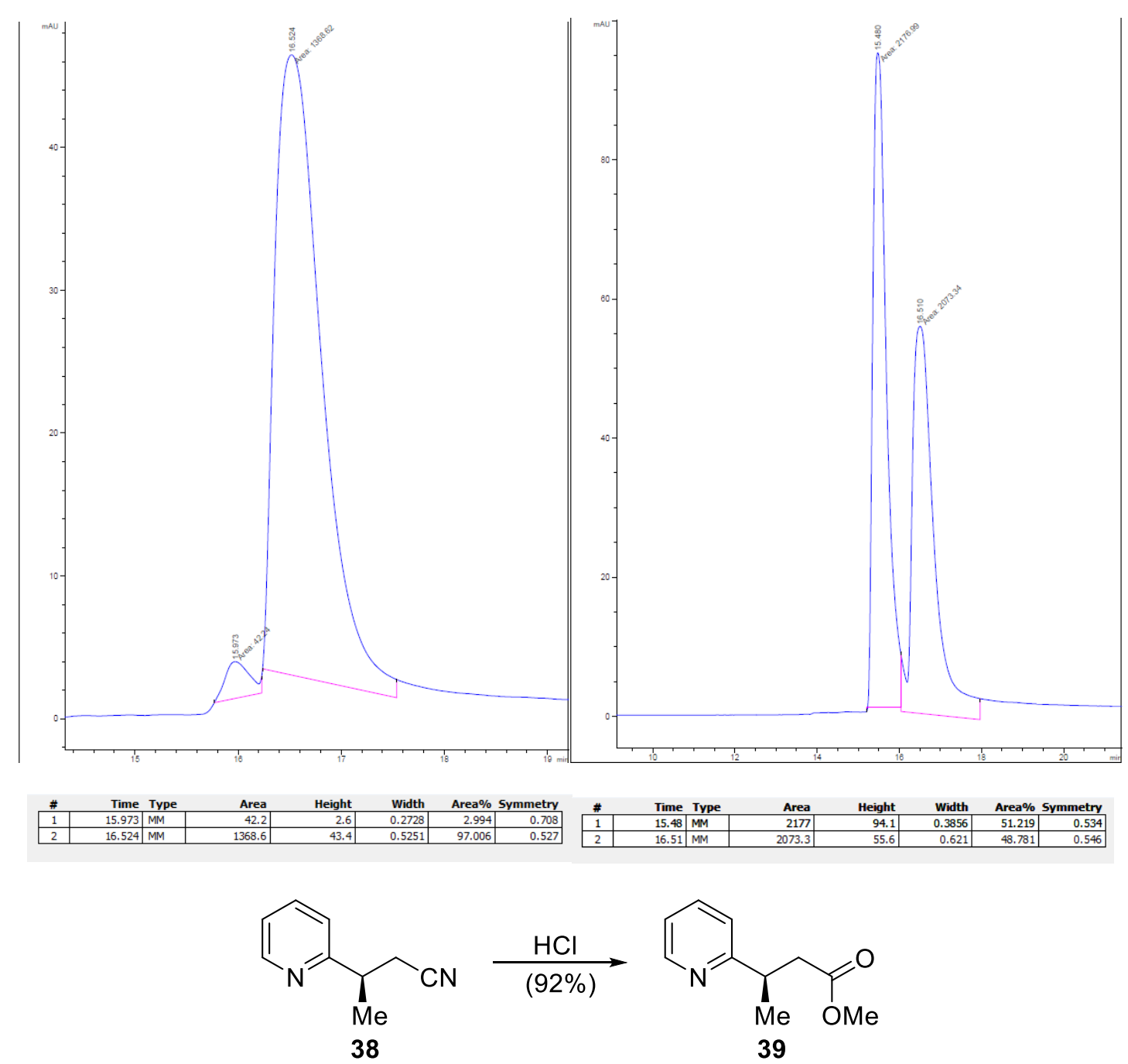

Methyl (R)-3-(pyridin-2-yl)butanoate (39): To a reaction tube containing a stir bar and nitrile 38 (2.52 $\mathrm{g}, 17.2 \mathrm{mmol}, 1.0$ equiv) at $22{ }^{\circ} \mathrm{C}$ was added a methanolic solution of hydrochloric acid (3.0 M, $\left.57 \mathrm{~mL}\right)$. The vessel was sealed, and the stirred reaction mixture was heated to $60{ }^{\circ} \mathrm{C}$, at which point the reaction vessel was briefly depressurized with an exit needle. After $4 \mathrm{~h}$, the reaction mixture was allowed to cool to $22{ }^{\circ} \mathrm{C}$ and was carefully neutralized with a saturated aqueous solution of sodium bicarbonate $(120 \mathrm{~mL})$. 


\section{Rhoades, * et al.}

The mixture was directly extracted with ethyl acetate $(3 \times 20 \mathrm{~mL})$, and the combined organic layers were dried over anhydrous sodium sulfate and concentrated under reduced pressure. The obtained residue was sufficiently pure for immediate use in the following step, but could also be purified by flash column chromatography (silica gel, 35\% ethyl acetate in hexanes) to afford ester 39 (2.83 g, $15.8 \mathrm{mmol}, 92 \%$ yield) as a colorless oil.

$\mathbf{R}_{f}=0.41$ (silica gel, 35\% ethyl acetate in hexanes, UV, potassium permanganate).

$[\boldsymbol{\alpha}]_{\mathbf{D}}^{22}=-21.4\left(c=0.70, \mathrm{CH}_{2} \mathrm{Cl}_{2}\right)$.

FT-IR (thin film): $v_{\max } 3056,3008,2968,2954,2931,2875,2847,1733,1592,1570,1474,1456,1435$, 1364, 1304, 1275, 1257, 1204, 1192, 1165, 1099, 1072, 1050, 1031, 1006, 991, 934, 900, 884, 841, 787, $749,713 \mathrm{~cm}^{-1}$.

${ }^{1} \mathbf{H}$ NMR $\left(600 \mathrm{MHz}, \mathbf{C D C l}_{3}\right): \delta 8.52(\mathrm{ddd}, J=4.8,1.8,0.8 \mathrm{~Hz}, 1 \mathrm{H}), 7.59(\mathrm{ddd}, J=7.8,7.5,1.8 \mathrm{~Hz}, 1$ H), 7.18 (ddd, $J=7.8,1.1,0.8 \mathrm{~Hz}, 1 \mathrm{H}), 7.10$ (ddd, $J=7.5,4.8,1.1 \mathrm{~Hz}, 1 \mathrm{H}), 3.61$ (s, $3 \mathrm{H}), 3.41$ (dqd, $J$ $=7.7,7.0,7.0 \mathrm{~Hz}, 1 \mathrm{H}), 2.88(\mathrm{dd}, J=15.8,7.5 \mathrm{~Hz}, 1 \mathrm{H}), 2.60(\mathrm{dd}, J=15.8,7.1 \mathrm{~Hz}, 1 \mathrm{H}), 1.32(\mathrm{~d}, J=7.0$ $\mathrm{Hz}, 3 \mathrm{H}) \mathrm{ppm}$.

${ }^{13}$ C NMR (151 MHz, CDCl $)$ ): $\delta$ 173.2, 164.5, 149.4, 136.6, 122.1, 121.6, 51.6, 40.7, 38.2, 20.9 ppm. HRMS (ESI, $\boldsymbol{m} / \boldsymbol{z}$ ): calcd for $\mathrm{C}_{10} \mathrm{H}_{14 \mathrm{NO}_{2}}{ }^{[}[\mathrm{M}+\mathrm{H}]^{+}$180.1019, found 180.1025, $\Delta 3.3 \mathrm{ppm}$.

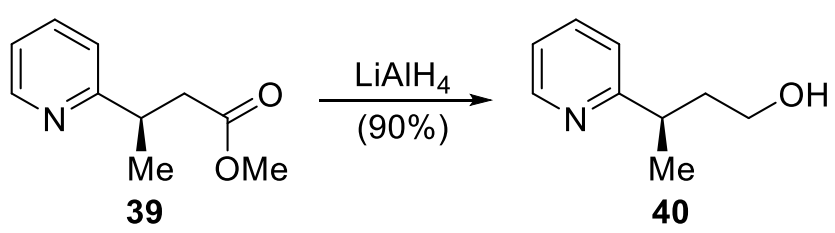

(R)-3-(Pyridin-2-yl)butan-1-ol (40): To a stirred solution of ester 39 (2.70 g, $15.1 \mathrm{mmol}, 1.0$ equiv) in tetrahydrofuran $(50 \mathrm{~mL})$ at $0{ }^{\circ} \mathrm{C}$ was added a solution of lithium aluminum hydride $(1.0 \mathrm{M}$ tetrahydrofuran, $15.1 \mathrm{~mL}, 15.1 \mathrm{mmol}, 1.0$ equiv) dropwise. After $30 \mathrm{~min}$, the reaction mixture was carefully quenched with water $(0.57 \mathrm{~mL})$, an aqueous solution of sodium hydroxide $(10 \% w / v, 0.57 \mathrm{~mL})$, and water $(1.71 \mathrm{~mL})$ sequentially, and was vigorously stirred for $10 \mathrm{~min}$. Then the two phases were 


\section{Rhoades, * et al.}

separated, and the aqueous layer was extracted with ethyl acetate $(3 \times 10 \mathrm{~mL})$. The combined organic layers were dried over anhydrous sodium sulfate and concentrated under reduced pressure. The obtained residue was purified by flash column chromatography (silica gel, dichloromethane $\rightarrow 10 \%$ methanol in dichloromethane) to afford alcohol 40 (2.06 g, $13.6 \mathrm{mmol}, 90 \%$ yield) as a colorless oil.

$\mathbf{R}_{f}=0.45$ (silica gel, 10\% methanol in dichloromethane, UV, potassium permanganate). $[\boldsymbol{\alpha}]_{\mathbf{D}}^{22}=-22.8\left(c=1.00, \mathrm{CH}_{2} \mathrm{Cl}_{2}\right)$.

FT-IR (thin film): $v_{\max } 3309,3084,3010,2962,2930,2872,1669,1594,1570,1476,1454,1434,1375$, $1288,1247,1212,1184,1151,1053,1044,1000,974,953,913,894,849,786,750,668 \mathrm{~cm}^{-1}$.

${ }^{1} \mathbf{H}$ NMR (600 MHz, CDCl$): ~ \delta 8.49(\mathrm{ddd}, J=4.9,1.8,0.9 \mathrm{~Hz}, 1 \mathrm{H}), 7.63(\mathrm{ddd}, J=7.9,7.5,1.8 \mathrm{~Hz}, 1$ H), 7.19 (ddd, $J=7.9,1.1,0.9 \mathrm{~Hz}, 1 \mathrm{H}), 7.12$ (ddd, $J=7.5,4.9,1.1 \mathrm{~Hz}, 1 \mathrm{H}), 3.77$ (br s, $1 \mathrm{H}$ ), 3.62 (ddd, $J=11.1,5.4,5.2 \mathrm{~Hz}, 1 \mathrm{H}), 3.57(\mathrm{ddd}, J=11.1,8.3,5.0 \mathrm{~Hz}, 1 \mathrm{H}), 3.17(\mathrm{dqd}, J=8.7,7.1,5.0 \mathrm{~Hz}, 1 \mathrm{H})$, 1.96 (dddd, $J=14.0,8.3,5.4,5.0 \mathrm{~Hz}, 1 \mathrm{H}), 1.88(\mathrm{dddd}, J=14.0,8.7,5.2,5.0 \mathrm{~Hz}, 1 \mathrm{H}), 1.33(\mathrm{~d}, J=7.1$ $\mathrm{Hz}, 3 \mathrm{H}) \mathrm{ppm}$.

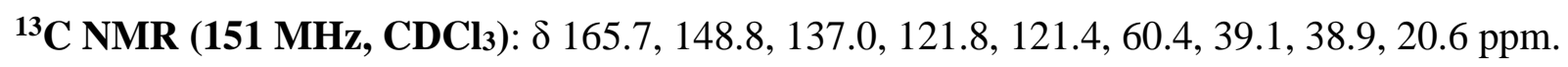

HRMS (ESI, $\boldsymbol{m} / z$ ): calcd for $\mathrm{C}_{9} \mathrm{H}_{14} \mathrm{NO}^{+}[\mathrm{M}+\mathrm{H}]^{+} 152.1070$, found $152.1068, \Delta 1.3 \mathrm{ppm}$.
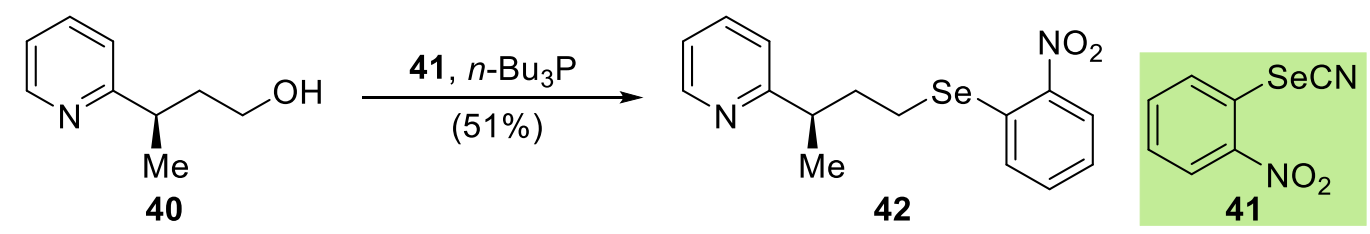

(R)-2-\{4-[(2-Nitrophenyl)selanyl]butan-2-yl\}pyridine (42): To a stirred solution of alcohol 40 (1.36 g, $8.99 \mathrm{mmol}, 1.0$ equiv) in tetrahydrofuran $(90 \mathrm{~mL})$ at $0{ }^{\circ} \mathrm{C}$ was added 2-nitrophenylselenocyanate (41) (4.09 g, $18.0 \mathrm{mmol}, 2.0$ equiv), followed by the dropwise addition of tri- $n$-butylphosphine ( $4.50 \mathrm{~mL}, 18.0$ mmol, 2.0 equiv). After $2 \mathrm{~h}$, the reaction mixture was quenched with a saturated aqueous solution of ammonium bicarbonate $(20 \mathrm{~mL})$ and water $(20 \mathrm{~mL})$, and allowed to warm to $22^{\circ} \mathrm{C}$. The two phases were separated, and the aqueous layer was extracted with ethyl acetate $(3 \times 15 \mathrm{~mL})$. The combined organic 


\section{Rhoades, * et al.}

layers were dried over anhydrous sodium sulfate and concentrated under reduced pressure. The obtained residue was filtered through a short silica pad, rinsed thoroughly with diethyl ether $(250 \mathrm{~mL})$, and concentrated under reduced pressure. The obtained residue was further purified by flash column chromatography (silica gel, $5 \rightarrow 25 \%$ diethyl ether in hexanes) to afford selenide 42 (1.54 g, 4.58 mmol, $51 \%$ yield) as a viscous orange oil.

$\mathbf{R}_{f}=0.27$ (silica gel, $40 \%$ ethyl acetate in hexanes, UV, potassium permanganate).

$[\boldsymbol{\alpha}]_{\mathbf{D}}^{22}=-14.3\left(c=0.30, \mathrm{CH}_{2} \mathrm{Cl}_{2}\right)$.

FT-IR (thin film): $v_{\max }$ 3080, 3006, 2963, 2925, 2853, 1590, 1566, 1541, 1511 1473, 1452, 1433, 1330, $1304,1250,1218,1193,1168,1150,1096,1050,1037,990,852,785,749,729,702,680 \mathrm{~cm}^{-1}$.

${ }^{1}$ H NMR (600 MHz, CDCl3): $\delta 8.59(\mathrm{ddd}, J=4.8,1.8,0.9 \mathrm{~Hz}, 1 \mathrm{H}), 8.27(\mathrm{ddd}, J=8.3,8.1,1.5 \mathrm{~Hz}, 1$ H), $7.63(\mathrm{ddd}, J=7.9,7.5,1.8 \mathrm{~Hz}, 1 \mathrm{H}), 7.46(\mathrm{ddd}, J=8.4,7.1,1.5 \mathrm{~Hz}, 1 \mathrm{H}), 7.41(\mathrm{ddd}, J=8.4,8.1,1.3$ $\mathrm{Hz}, 1 \mathrm{H}), 7.28(\mathrm{ddd}, J=8.3,7.1,1.4 \mathrm{~Hz}, 1 \mathrm{H}), 7.19(\mathrm{ddd}, J=7.9,1.1,0.9 \mathrm{~Hz}, 1 \mathrm{H}), 7.15$ (ddd, $J=7.5$, 4.8, 1.1 Hz, $1 \mathrm{H}), 3.08$ (dqd, $J=9.0,7.0,5.5 \mathrm{~Hz}, 1 \mathrm{H}), 2.82(\mathrm{ddd}, J=12.0,9.7,5.8 \mathrm{~Hz}, 1 \mathrm{H}), 2.78$ (ddd, $J=12.0,9.2,6.7 \mathrm{~Hz}, 1 \mathrm{H}), 2.30(\mathrm{dddd}, J=13.9,9.2,9.0,5.8 \mathrm{~Hz}, 1 \mathrm{H}), 2.07$ (dddd, $J=13.9,9.7,6.7,5.5$ $\mathrm{Hz}, 1 \mathrm{H}), 1.35$ (d, $J=7.0 \mathrm{~Hz}, 3 \mathrm{H}) \mathrm{ppm}$.

${ }^{13}$ C NMR (151 MHz, CDCl3): $\delta$ 164.9, 149.6, 147.0, 136.7, 133.8, 133.7, 129.2, 126.6, 125.4, 122.4, 121.7, 42.4, 35.5, 24.2, $21.1 \mathrm{ppm}$.

HRMS (ESI, $\boldsymbol{m} / z$ ): calcd for $\mathrm{C}_{15} \mathrm{H}_{17} \mathrm{~N}_{2} \mathrm{O}_{2} \mathrm{Se}^{+}[\mathrm{M}+\mathrm{H}]^{+}$337.0450, found 337.0458, $\Delta 2.4$ ppm.

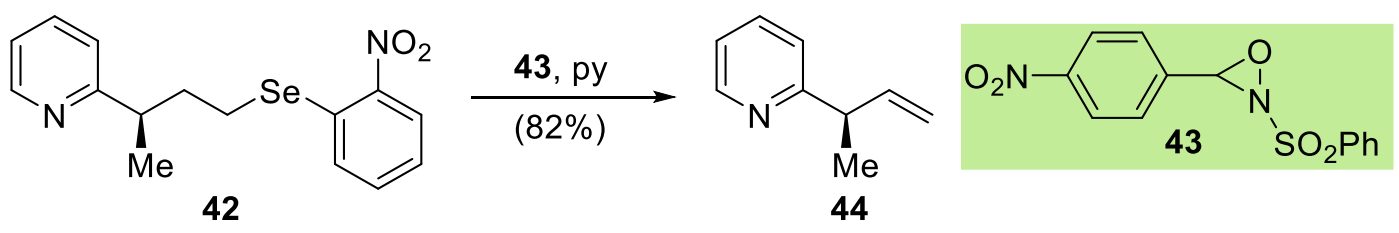

(R)-2-(But-3-en-2-yl)pyridine (44): To a stirred solution of selenide 42 (846 mg, 2.52 mmol, 1.0 equiv) in chloroform $(3.8 \mathrm{~mL})$ at $0{ }^{\circ} \mathrm{C}$ was added pyridine $(1.00 \mathrm{~mL}, 12.6 \mathrm{mmol}, 5.0$ equiv), followed by oxaziridine 43 (772 mg, $2.52 \mathrm{mmol}, 1.0$ equiv), and the reaction mixture was allowed to slowly warm to 


\section{Rhoades, * et al.}

$22{ }^{\circ} \mathrm{C}$. After 6 hours, the reaction mixture was filtered to remove the sulfonimine byproduct, and the filtrate was carefully concentrated at $0{ }^{\circ} \mathrm{C}$ under reduced pressure (170-160 torr). The obtained residue was purified by flash column chromatography (pentanes $\rightarrow 10 \%$ diethyl ether in pentanes) to afford allylic pyridine 44 (275 mg, $2.07 \mathrm{mmol}, 82 \%$ yield) as a colorless, odiferous oil with residual solvent. Concentration to dryness results in substantial material loss. An analytically pure sample was obtained by allowing an aliquot of the purified solution to slowly evaporate to dryness in a loosely sealed flask.

$\mathbf{R}_{f}=0.37$ (silica gel, $40 \%$ diethyl ether in pentanes, UV, potassium permanganate).

$[\boldsymbol{\alpha}]_{\mathbf{D}}^{22}=-20.8\left(c=0.50, \mathrm{CHCl}_{3}\right)$.

FT-IR (thin film): $v_{\max } 3079,3006,2965,2925,2853,1638,1590,1569,1554,1520,1472,1433,1365$, $1333,1306,1203,1149,1032,1018,993,914,857,800,780,748,732,685 \mathrm{~cm}^{-1}$.

${ }^{1} \mathbf{H}$ NMR (600 MHz, CDCl3): $\delta 8.55(\mathrm{ddd}, J=4.8,1.9,0.9 \mathrm{~Hz}, 1 \mathrm{H}), 7.61(\mathrm{ddd}, 7.9,7.5,1.9 \mathrm{~Hz}, 1 \mathrm{H})$, $7.17(\mathrm{ddd}, J=7.9,1.2,0.9 \mathrm{~Hz}, 1 \mathrm{H}), 7.11$, (ddd, $J=7.5,4.8,1.2 \mathrm{~Hz}, 1 \mathrm{H}), 6.09$ (ddd, $J=17.2,10.3,6.8$ Hz, $1 \mathrm{H}), 5.12$ (ddd, $J=17.2,1.5,1.5 \mathrm{~Hz}, 1 \mathrm{H}), 5.09$ (ddd, $J=10.3,1.4,1.4 \mathrm{~Hz}, 1 \mathrm{H}), 3.64$ (qddd, $J=7.1$, 6.8, 1.5, $1.4 \mathrm{~Hz}, 1 \mathrm{H}), 1.42(\mathrm{~d}, J=7.1 \mathrm{~Hz}, 3 \mathrm{H}) \mathrm{ppm}$.

${ }^{13}$ C NMR (151 MHz, CDCl3): $\delta$ 164.8, 149.4, 142.0, 136.6, 121.7, 121.4, 114.2, 45.9, 19.9 ppm.

HRMS (ESI, $\boldsymbol{m} / z)$ ): calcd for $\mathrm{C}_{9} \mathrm{H}_{12} \mathrm{~N}^{+}[\mathrm{M}+\mathrm{H}]^{+}$134.0964, found 134.0962, $\Delta 1.5 \mathrm{ppm}$.
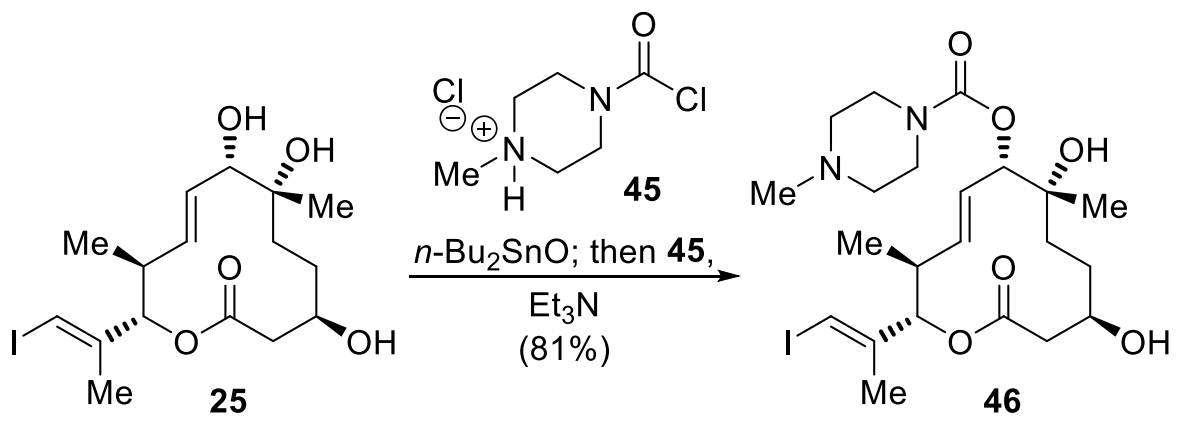

$(2 S, 3 S, 6 S, 7 R, 10 R, E)-7,10-D i h y d r o x y-2-[(E)-1-i o d o p r o p-1-e n-2-y l]-3,7-d i m e t h y l-12-$

oxooxacyclododec-4-en-6-yl 4-methylpiperazine-1-carboxylate (46): To a reaction tube containing a stir bar and activated $4 \AA$ molecular sieves $(150 \mathrm{mg})$ under argon at $22{ }^{\circ} \mathrm{C}$ was added dibutyltin oxide 


\section{Rhoades, * et al.}

(32.4 $\mathrm{mg}, 0.130 \mathrm{mmol}, 1.1$ equiv) and toluene ( $3.8 \mathrm{~mL}$ ) with stirring, followed by a solution of $\mathbf{2 5}$ (50.0 $\mathrm{mg}, 0.118 \mathrm{mmol}, 1.0$ equiv) in toluene $(1.2 \mathrm{~mL})$, and the reaction vessel was sealed and heated to $100{ }^{\circ} \mathrm{C}$. After 12 hours, the reaction mixture was cooled to $80{ }^{\circ} \mathrm{C}$, triethylamine $(0.06 \mathrm{~mL}, 0.4 \mathrm{mmol}, 3.4$ equiv) and 4-methyl-1-piperazinecarbonyl chloride hydrochloride (45) (60 mg, $0.3 \mathrm{mmol}, 2.5$ equiv) were added sequentially, and stirring was continued for an additional 36 hours. Then the reaction mixture was allowed to cool to $22{ }^{\circ} \mathrm{C}$, filtered through a celite pad, and rinsed thoroughly with ethyl acetate $(15 \mathrm{~mL})$ and $10 \%$ methanol in dichloromethane $(3 \mathrm{~mL})$. The filtrate was washed with a saturated aqueous solution of sodium bicarbonate $(15 \mathrm{~mL})$, and the organic layer was dried over anhydrous sodium sulfate and concentrated under reduced pressure. The obtained residue was purified by flash column chromatography (silica gel, dichloromethane $\rightarrow 2 \%$ methanol in dichloromethane $\rightarrow 10 \%$ methanol in dichloromethane) to afford carbamate 46 (53 mg, $96 \mu \mathrm{mol}, 81 \%$ yield) as a colorless oil.

$\mathbf{R}_{f}=0.30$ (silica gel, 10\% methanol in dichloromethane, UV, $p$-anisaldehyde).

$[\boldsymbol{\alpha}]_{\mathbf{D}}^{22}=-54.5\left(c=0.20, \mathrm{CH}_{2} \mathrm{Cl}_{2}\right)$.

FT-IR (thin film): $v_{\max } 3426,3056,2925,2854,2797,1730,1699,1681,1617,1459,1431,1376,1338$, $1292,1261,1239,1218,1156,1103,1062,1053,1016,1004,980,965,943,905,837,789,672 \mathrm{~cm}^{-1}$. ${ }^{1} \mathbf{H}$ NMR (600 MHz, CDCl 3$): \delta 6.48($ br q, $J=1.1 \mathrm{~Hz}, 1 \mathrm{H}), 5.71(\mathrm{dd}, J=15.2,9.8 \mathrm{~Hz}, 1 \mathrm{H}), 5.58(\mathrm{dd}, J$ $=15.2,10.0 \mathrm{~Hz}, 1 \mathrm{H}), 5.30(\mathrm{~d}, J=10.7 \mathrm{~Hz}, 1 \mathrm{H}), 5.01(\mathrm{~d}, J=9.8 \mathrm{~Hz}, 1 \mathrm{H}), 3.77-3.75(\mathrm{~m}, 1 \mathrm{H}), 3.51-3.50$ (m, $4 \mathrm{H}), 2.64(\mathrm{dd}, J=15.0,3.7 \mathrm{~Hz}, 1 \mathrm{H}), 2.54(\mathrm{dd}, J=15.0,3.1 \mathrm{~Hz}, 1 \mathrm{H}), 2.50(\mathrm{ddq}, J=10.7,10.0,6.8$ $\mathrm{Hz}, 1 \mathrm{H}), 2.42-2.35$ (m, 4 H), 2.31 (br s, $3 \mathrm{H}), 1.82$ (d, $J=1.1 \mathrm{~Hz}, 3 \mathrm{H}), 1.71$ (br s, $2 \mathrm{H}), 1.70$ (app tt, $J=$ 13.5, $3.8 \mathrm{~Hz}, 1 \mathrm{H}), 1.52(\operatorname{app} t d, J=14.2,3.4 \mathrm{~Hz}, 1 \mathrm{H}), 1.38(\operatorname{app} t d, J=14.2,4.2 \mathrm{~Hz}, 1 \mathrm{H}), 1.31-1.27(\mathrm{~m}$, $1 \mathrm{H}), 1.23(\mathrm{br} \mathrm{s}, 3 \mathrm{H}), 0.91(\mathrm{~d}, J=6.8 \mathrm{~Hz}, 3 \mathrm{H}) \mathrm{ppm}$.

${ }^{13}$ C NMR (151 MHz, CDCl) : $\delta$ 171.9, 154.4, 143.5, 139.3, 126.8, 84.3, 80.4, 80.0, 74.0, 69.3, 54.8, 46.3, 44.0, 41.1, 38.5, 35.5, 30.0, 24.9, 19.2, $16.5 \mathrm{ppm}$.

HRMS (ESI, $\boldsymbol{m} / \mathbf{z}$ ): calcd for $\mathrm{C}_{22} \mathrm{H}_{36} \mathrm{IN}_{2} \mathrm{O}_{6}{ }^{+}[\mathrm{M}+\mathrm{H}]^{+}$551.1613, found 551.1607, $\Delta 1.1 \mathrm{ppm}$. 
D. Rhoades, * et al.
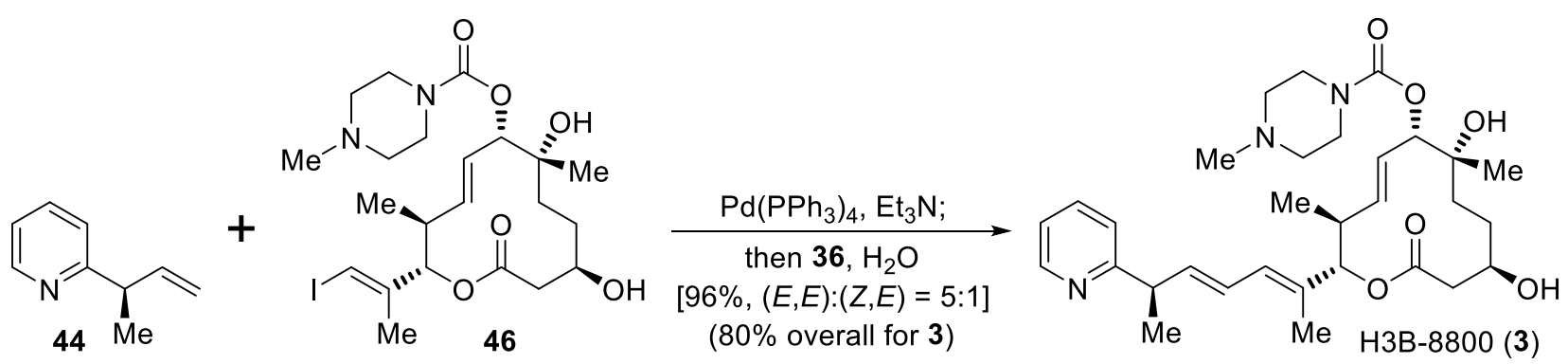

Table S7. Optimization of Mizoroki-Heck coupling of $\mathbf{4 4}$ and $\mathbf{4 6}$ to afford H3B-8800 (3).

\begin{tabular}{|c|c|c|c|}
\hline entry & conditions $^{\mathrm{a}}$ & yield $^{\mathrm{b}}$ & $(E, E):(Z, E)^{\mathrm{c}}$ \\
\hline 1 & $\mathrm{Pd}(\mathrm{OAc})_{2}(10 \mathrm{~mol} \%), \mathrm{P}\left(o-\mathrm{tol}_{3}\right)_{3}(20 \mathrm{~mol} \%), \mathrm{Et}_{3} \mathrm{~N}$ (3.0 equiv), DMF, $80^{\circ} \mathrm{C}, 4 \mathrm{~h}$ & $48 \%{ }^{\mathrm{d}}$ & $3: 4$ \\
\hline 2 & $\mathrm{Pd}(\mathrm{OAc})_{2}(10 \mathrm{~mol} \%), \mathrm{P}(o \text {-tol })_{3}(20 \mathrm{~mol} \%), \mathrm{Et}_{3} \mathrm{~N}$ (3.0 equiv), DMF, $80^{\circ} \mathrm{C}, 12 \mathrm{~h}$ & $70 \%{ }^{\mathrm{d}}$ & $3: 4$ \\
\hline 3 & $\mathrm{Pd}(\mathrm{OAc})_{2}(10 \mathrm{~mol} \%), \mathrm{P}(o \text {-tol })_{3}\left(20 \mathrm{~mol}^{\circ}\right), \mathrm{Et}_{3} \mathrm{~N}$ (3.0 equiv), $\mathrm{MeCN}, 80^{\circ} \mathrm{C}, 4 \mathrm{~h}$ & $60 \%{ }^{\mathrm{d}}$ & $1: 2$ \\
\hline 4 & $\mathrm{Pd}(\mathrm{OAc})_{2}(10 \mathrm{~mol} \%), \mathrm{P}\left(o-\text { tol }_{3}\right)_{3}(20 \mathrm{~mol} \%), \mathrm{Et}_{3} \mathrm{~N}$ (3.0 equiv), $\mathrm{MeCN}, 80^{\circ} \mathrm{C}, 12 \mathrm{~h}$ & $60 \%{ }^{\mathrm{d}}$ & $1: 2$ \\
\hline 5 & Herrmann-Beller palladacycle ( $10 \mathrm{~mol} \%), \mathrm{Et}_{3} \mathrm{~N}$ (3.0 equiv), DMF, $80^{\circ} \mathrm{C}, 4 \mathrm{~h}$ & $67 \%{ }^{\mathrm{d}}$ & $3: 4$ \\
\hline 6 & Herrmann-Beller palladacycle (10 mol\%), Et ${ }_{3} \mathrm{~N}$ (3.0 equiv), DMF, $80^{\circ} \mathrm{C}, 12 \mathrm{~h}$ & $77 \%{ }^{\mathrm{d}}$ & $3: 4$ \\
\hline 7 & Herrmann-Beller palladacycle ( $25 \mathrm{~mol} \%$ ), $\mathrm{Et}_{3} \mathrm{~N}$ (3.0 equiv), DMF, $80^{\circ} \mathrm{C}, 4 \mathrm{~h}$ & $84 \%$ & $3: 4$ \\
\hline 8 & Herrmann-Beller palladacycle (10 mol\%), $\mathrm{Et}_{3} \mathrm{~N}$ (3.0 equiv), $\mathrm{MeCN}, 80^{\circ} \mathrm{C}, 4 \mathrm{~h}$ & $60 \%{ }^{\mathrm{d}}$ & $1: 2$ \\
\hline 9 & $\mathrm{Pd}\left(\mathrm{PPh}_{3}\right)_{4}(10 \mathrm{~mol} \%), \mathrm{Et}_{3} \mathrm{~N}$ (3.0 equiv), DMF, $80{ }^{\circ} \mathrm{C}, 4 \mathrm{~h}$ & $85 \%$ & $2: 1$ \\
\hline 10 & $\mathrm{Pd}\left(\mathrm{PPh}_{3}\right)_{4}(10 \mathrm{~mol} \%), \mathrm{Et}_{3} \mathrm{~N}$ (3.0 equiv), $\mathrm{MeCN}, 80^{\circ} \mathrm{C}, 4 \mathrm{~h}$ & $80 \%$ & $3: 1$ \\
\hline 11 & $\mathrm{Pd}\left(\mathrm{PPh}_{3}\right)_{4}(10 \mathrm{~mol} \%), \mathrm{Et}_{3} \mathrm{~N}$ (3.0 equiv), $\mathrm{PhH}, 80^{\circ} \mathrm{C}, 4 \mathrm{~h}$ & $90 \%$ & $5: 1$ \\
\hline 12 & $\mathrm{Pd}\left(\mathrm{PPh}_{3}\right)_{4}(10 \mathrm{~mol} \%), \mathrm{Et}_{3} \mathrm{~N}$ (3.0 equiv), $\mathrm{PhMe}, 100{ }^{\circ} \mathrm{C}, 4 \mathrm{~h}$ & $74 \%$ & $5: 1$ \\
\hline $13^{\mathrm{e}}$ & $\mathrm{Pd}\left(\mathrm{PPh}_{3}\right)_{4}(10 \mathrm{~mol} \%), \mathrm{Et}_{3} \mathrm{~N}$ (3.0 equiv), $\mathrm{PhH}, 80^{\circ} \mathrm{C}, 4 \mathrm{~h}$ & $96 \%$ & $5: 1$ \\
\hline
\end{tabular}

${ }^{\mathrm{a}}$ All reactions were performed on a $5.5 \mu \mathrm{mol}(3.0 \mathrm{mg})$ scale based on 1.0 equiv of $\mathbf{4 6}$, unless otherwise indicated.

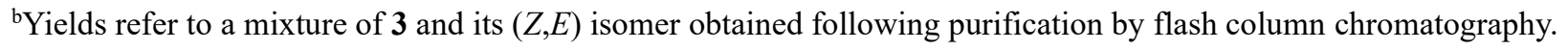

cProduct distributions were measured by ${ }^{1} \mathrm{H}$ NMR spectroscopic analysis.

${ }^{\mathrm{d}}$ Incomplete conversion of $\mathbf{4 6}$ was observed.

${ }^{\mathrm{e} R e a c t i o n}$ was performed on a $47 \mu \mathrm{mol}(26 \mathrm{mg})$ scale.

$(2 S, 3 S, 6 S, 7 R, 10 R, E)-7,10-D i h y d r o x y-3,7-d i m e t h y l-12-0 x o-2-[(R, 2 E, 4 E)$-6-(pyridin-2-yl)hepta-2,4-

dien-2-yl]oxacyclododec-4-en-6-yl 4-methylpiperazine-1-carboxylate (3): To a stirred solution of vinyl iodide 46 ( $26 \mathrm{mg}, 47 \mu \mathrm{mol}, 1.0$ equiv) and allylic pyridine 44 (19 mg, $140 \mu \mathrm{mol}, 3.0$ equiv) in benzene at $22{ }^{\circ} \mathrm{C}$ was added triethylamine $(19 \mu \mathrm{L}, 140 \mu$ mol, 3.0 equiv), followed by tetrakis(triphenylphosphine)palladium(0) $(5.4 \mathrm{mg}, 4.7 \mu \mathrm{mol}, 0.1$ equiv), and the reaction mixture was heated to $80{ }^{\circ} \mathrm{C}$. After $4 \mathrm{~h}$, the reaction mixture was allowed to cool to $22{ }^{\circ} \mathrm{C}$, ammonium pyrrolidinedithiocarbamate $36(3.9 \mathrm{mg}, 24 \mu \mathrm{mol}, 0.5$ equiv) and water $(0.010 \mathrm{~mL}, 0.55 \mathrm{mmol}, 12$ equiv) were added, and stirring was continued for $1 \mathrm{~h}$. Then the reaction mixture was filtered through a layer of 


\section{Rhoades, * et al.}

celite, rinsed thoroughly with ethyl acetate $(20 \mathrm{~mL})$ and $10 \%$ methanol in dichloromethane $(3 \mathrm{~mL})$, and concentrated under reduced pressure. The obtained residue was purified by flash column chromatography (silica gel, dichloromethane $\rightarrow 2 \%$ methanol in dichloromethane $\rightarrow 10 \%$ methanol in dichloromethane) to afford 3 [25 mg, $45 \mu \mathrm{mol}, 96 \%$ yield, $(E: E) /(Z: E)=5: 1,80 \%$ overall yield for 3] as a colorless film. An analytically pure sample was obtained via preparative thin layer chromatography (10\% methanol in acetone).

$\mathbf{R}_{f}=0.15$ (silica gel, $10 \%$ triethylamine in ethyl acetate, UV, $p$-anisaldehyde).

$[\boldsymbol{\alpha}]_{\mathrm{D}}^{22}=-14.0\left(c=0.20, \mathrm{CH}_{2} \mathrm{Cl}_{2}\right)$

FT-IR (thin film): $v_{\max } 3439,3051,2961,2933,2871,2796,1696,1684,1589,1570,1459,1432,1373$, $1340,1292,1261,1238,1217,1174,1153,1103,1063,1051,1004,978,960,930,875,837,786,763$ $732 \mathrm{~cm}^{-1}$.

${ }^{1}$ H NMR (600 MHz, CDCl3): $\delta 8.55(\mathrm{ddd}, J=4.8,1.9,0.9 \mathrm{~Hz}, 1 \mathrm{H}), 7.61(\mathrm{ddd}, 7.9,7.5,1.9 \mathrm{~Hz}, 1 \mathrm{H})$, $7.16(\mathrm{ddd}, J=7.9,1.2,0.9 \mathrm{~Hz}, 1 \mathrm{H}), 7.12$, $(\mathrm{ddd}, J=7.5,4.8,1.2 \mathrm{~Hz}, 1 \mathrm{H}), 6.32(\mathrm{ddd}, J=15.1,10.8,1.0$ Hz, $1 \mathrm{H}), 6.11(\mathrm{br} \mathrm{d}, J=10.8 \mathrm{~Hz}, 1 \mathrm{H}), 6.00(\mathrm{dd}, J=15.1,7.5 \mathrm{~Hz}, 1 \mathrm{H}), 5.68(\mathrm{dd}, J=15.2,9.7 \mathrm{~Hz}, 1 \mathrm{H})$, $5.60(\mathrm{dd}, J=15.2,9.8 \mathrm{~Hz}, 1 \mathrm{H}), 5.15(\mathrm{~d}, J=10.7 \mathrm{~Hz}, 1 \mathrm{H}), 5.01(\mathrm{~d}, J=9.7 \mathrm{~Hz}, 1 \mathrm{H}), 3.77-3.72(\mathrm{~m}, 1 \mathrm{H})$ $3.71(\mathrm{dqd}, J=7.5,7.0,1.0 \mathrm{~Hz}, 1 \mathrm{H}), 3.51-3.49(\mathrm{~m}, 4 \mathrm{H}), 2.61(\mathrm{dd}, J=14.9,3.5 \mathrm{~Hz}, 1 \mathrm{H}), 2.53(\mathrm{dd}, J=$ 14.9, $2.7 \mathrm{~Hz}, 1 \mathrm{H}), 2.51$ (ddq, $J=10.7,9.8,6.8 \mathrm{~Hz}, 1 \mathrm{H}), 2.43-2.36$ (m, $4 \mathrm{H}), 2.30$ (br s, $3 \mathrm{H}), 1.94$ (br s, $1 \mathrm{H}), 1.73$ (br s, $3 \mathrm{H}), 1.70(\operatorname{app} t \mathrm{t}, J=13.3,3.8 \mathrm{~Hz}, 1 \mathrm{H}), 1.54($ br s, $1 \mathrm{H}), 1.53(\operatorname{app} \mathrm{td}, J=14.0,3.2 \mathrm{~Hz}$ $1 \mathrm{H}), 1.44(\mathrm{~d}, J=7.0 \mathrm{~Hz}, 3 \mathrm{H}), 1.37(\operatorname{app} t d, J=14.0,3.4 \mathrm{~Hz}, 1 \mathrm{H}), 1.31(\operatorname{app} t \mathrm{tt}, J=13.3,2.7 \mathrm{~Hz}, 1 \mathrm{H})$, 1.23 (br s, $3 \mathrm{H}), 0.88$ (d, J=6.8 Hz, $3 \mathrm{H}) \mathrm{ppm}$.

${ }^{13}$ C NMR (151 MHz, CDCl3): $\delta$ 172.2, 164.6, 154.5, 149.5, 140.2, 139.9, 136.8, 131.35, 131.31, 126.1, $124.8,121.7,121.6,82.9,80.2,74.0,69.4,54.9,46.3,45.5,44.1,40.9,38.5,35.5,30.0,24.9,20.5,16.7$ $11.9 \mathrm{ppm}$.

HRMS (ESI, $\boldsymbol{m} / z)$ ): calcd for $\mathrm{C}_{31} \mathrm{H}_{46} \mathrm{~N}_{3} \mathrm{O}_{6}{ }^{+}[\mathrm{M}+\mathrm{H}]^{+}$556.3381, found 556.3382, $\Delta 0.2 \mathrm{ppm}$. 
D. Rhoades, * et al.

V. Comparison of Spectroscopic Data of Natural and Synthetic Pladienolides (A) and (B)

Natural Pladienolide A (1) ${ }^{1} \mathrm{H}$ NMR $\left(600 \mathrm{MHz}, \mathrm{CD}_{3} \mathrm{OD}\right)$

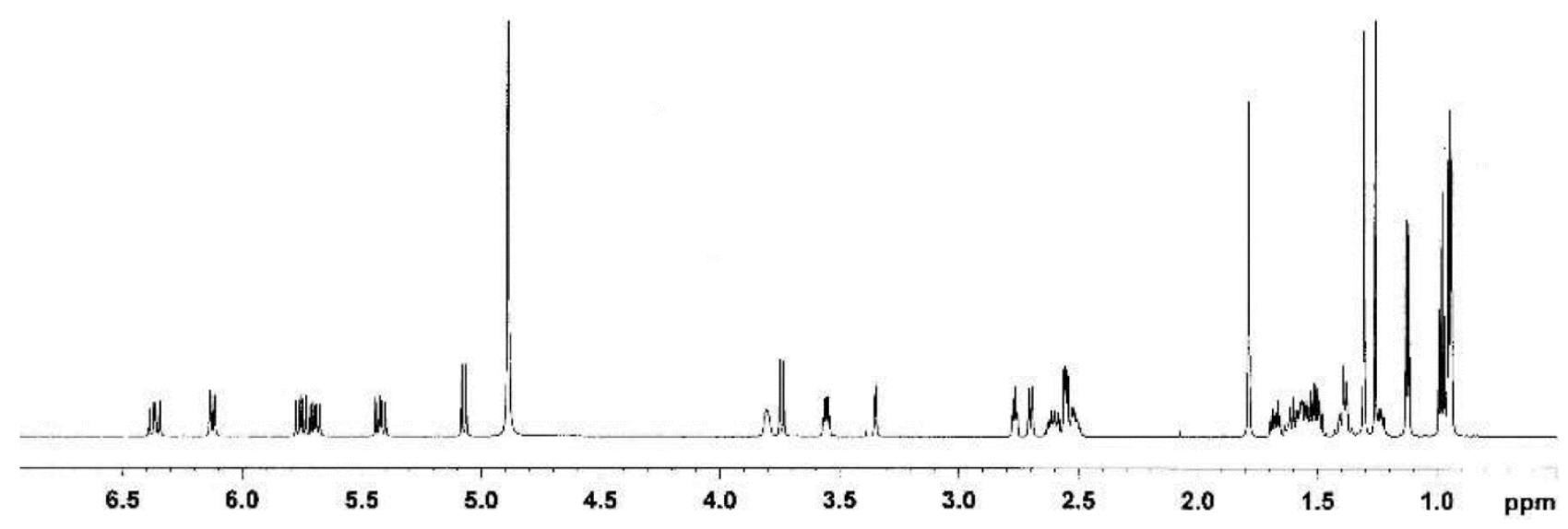

Synthetic Pladienolide A (1) ${ }^{1} \mathrm{H}$ NMR $\left(600 \mathrm{MHz}, \mathrm{CD}_{3} \mathrm{OD}\right)$

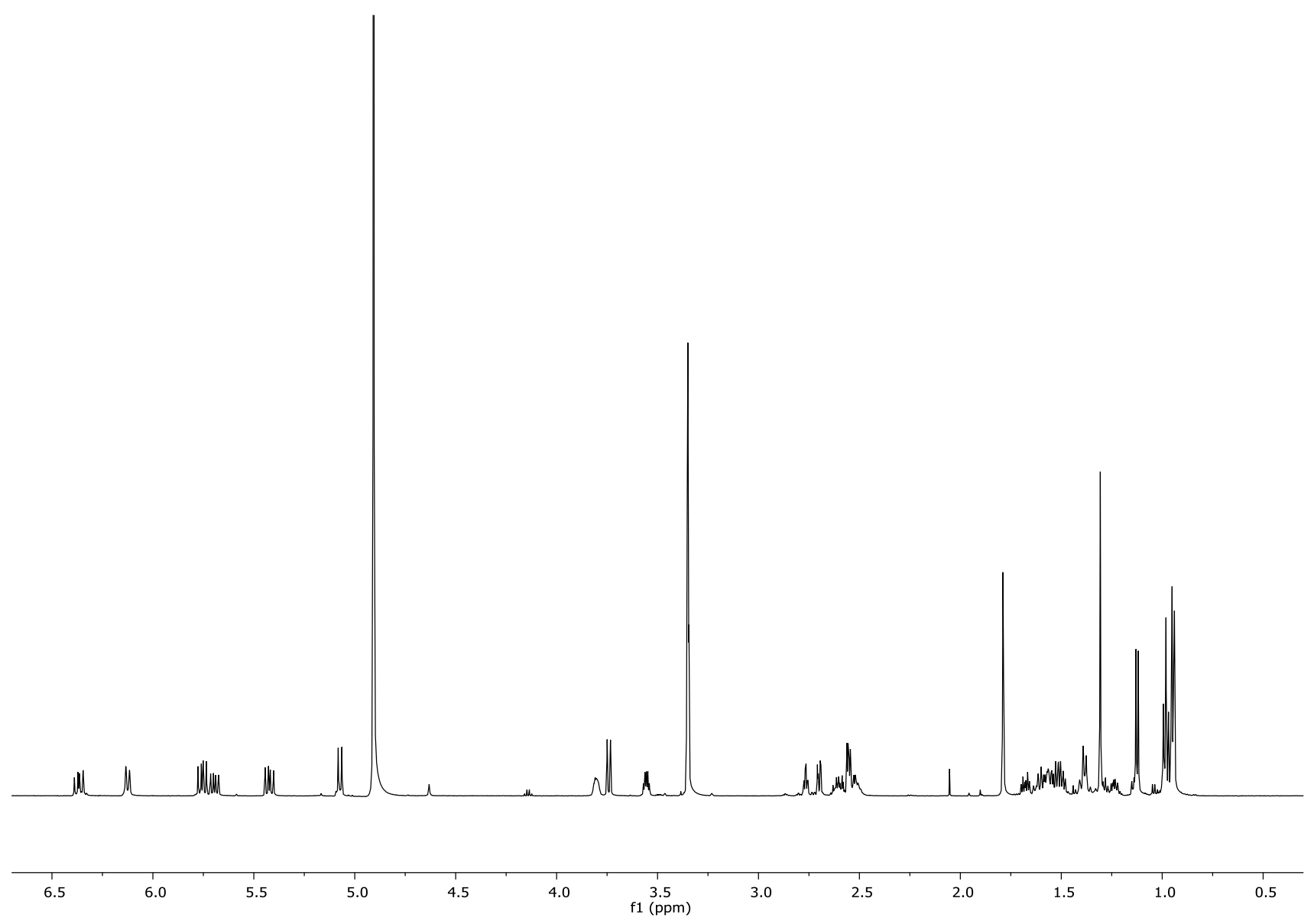


D. Rhoades, * et al.

Natural Pladienolide A (1) ${ }^{13} \mathrm{C}$ NMR (151 MHz, CD 3 OD)

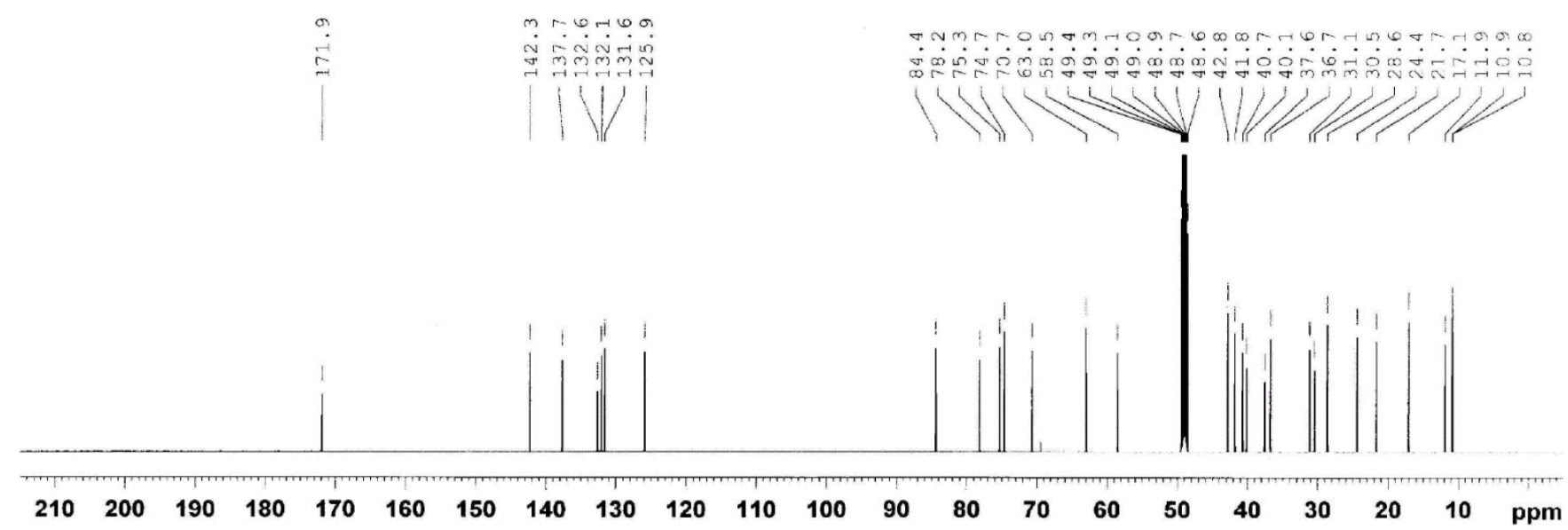

Synthetic Pladienolide A (1) ${ }^{13} \mathrm{C}$ NMR (151 MHz, CD ${ }_{3} \mathrm{OD}$ )

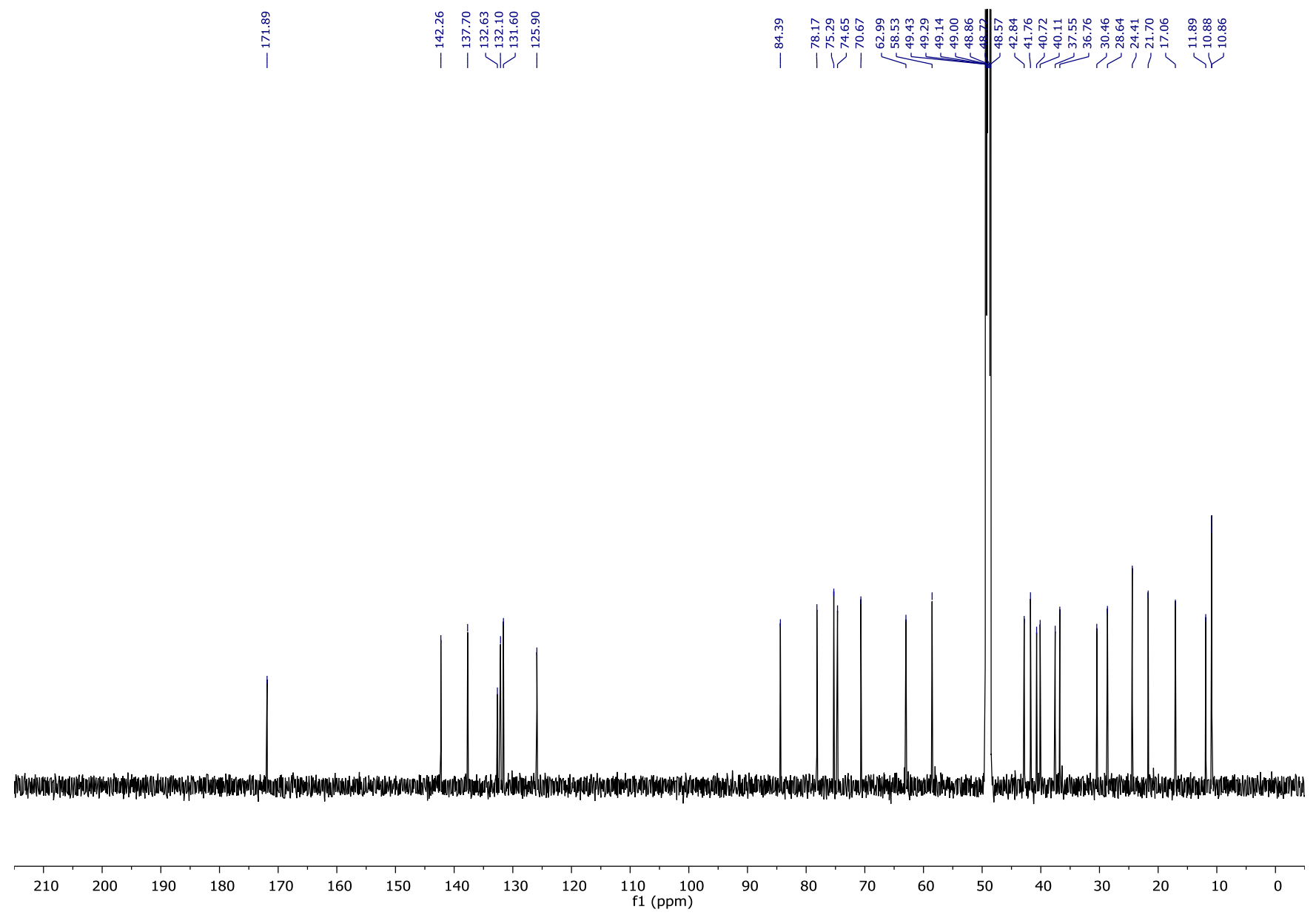


D. Rhoades, * et al.

Natural Pladienolide B (2) ${ }^{1} \mathrm{H}$ NMR $\left(600 \mathrm{MHz}, \mathrm{CD}_{3} \mathrm{OD}\right)$

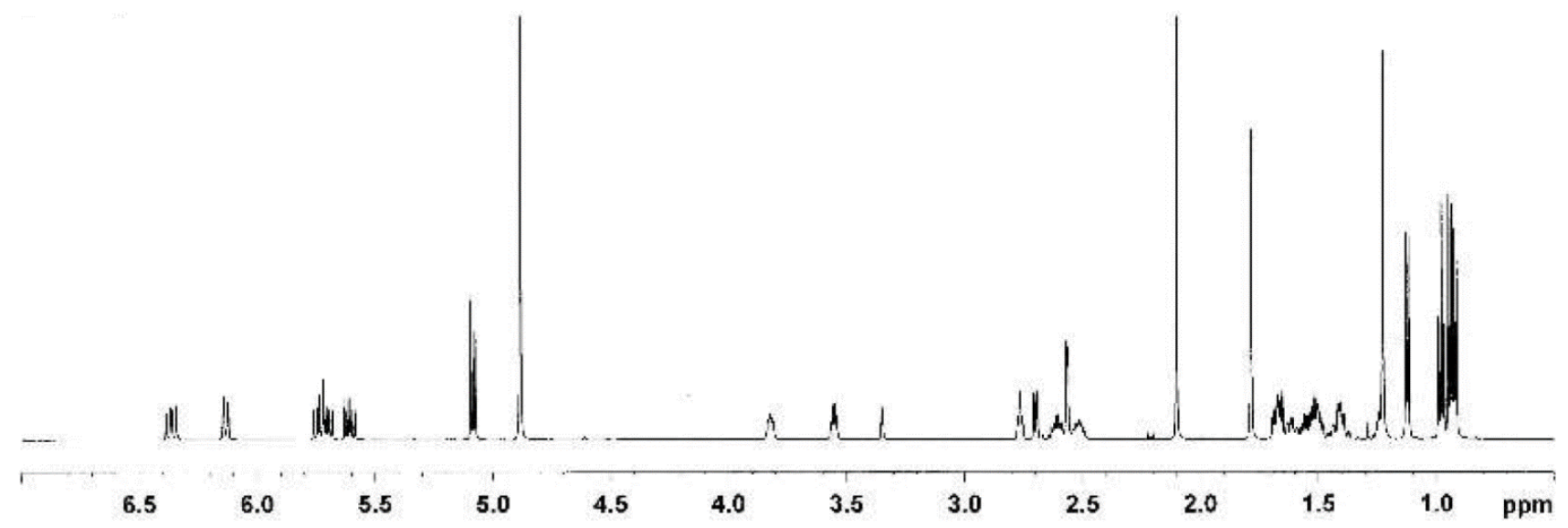

Synthetic Pladienolide B (2) ${ }^{1} \mathrm{H}$ NMR $\left(600 \mathrm{MHz}, \mathrm{CD}_{3} \mathrm{OD}\right)$

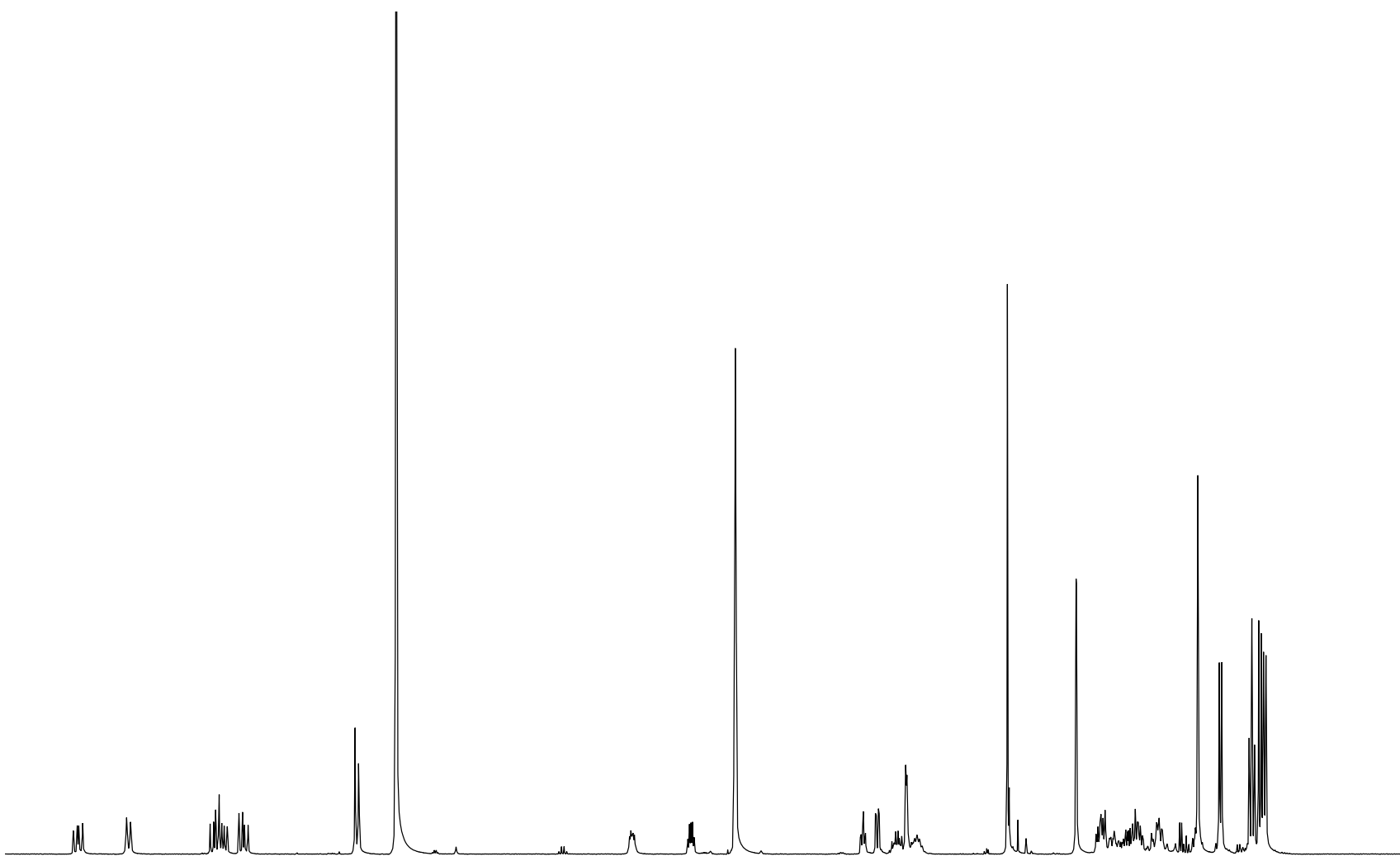

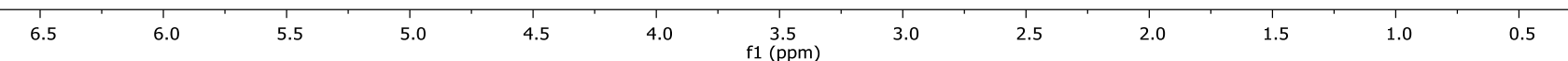


D. Rhoades, * et al.

Natural Pladienolide B (2) ${ }^{13} \mathrm{C}$ NMR (151 MHz, $\left.\mathrm{CD}_{3} \mathrm{OD}\right)$

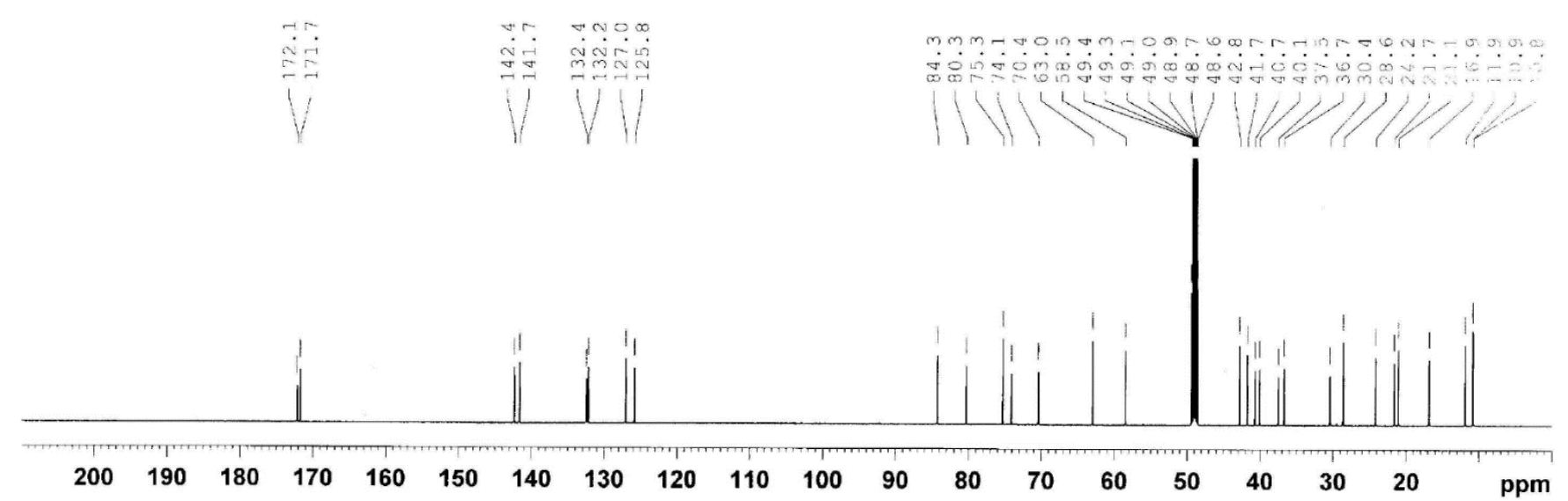

Synthetic Pladienolide B (2) ${ }^{13} \mathrm{C} N M R\left(151 \mathrm{MHz}, \mathrm{CD}_{3} \mathrm{OD}\right)$

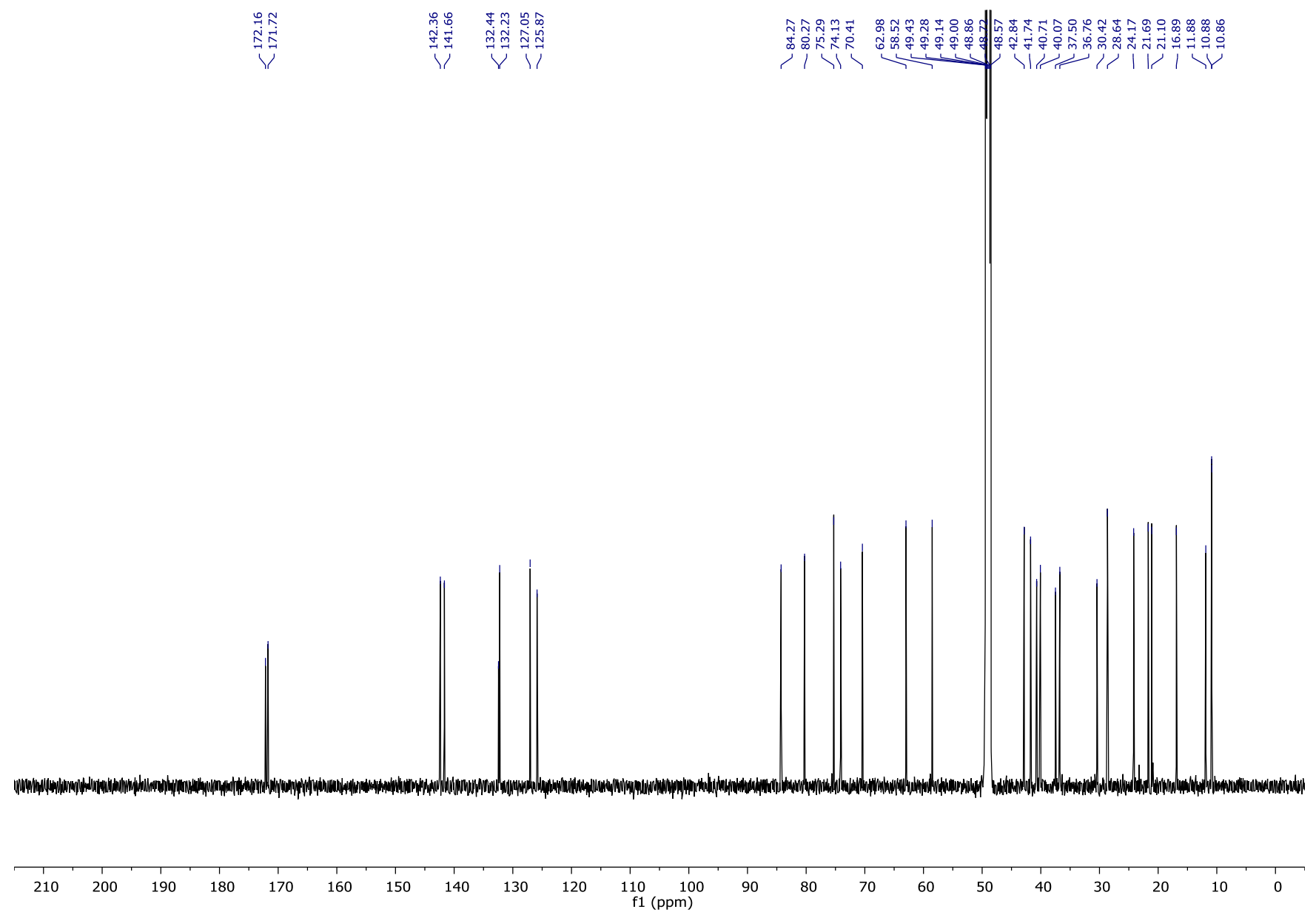




\section{Rhoades, * et al.}

\section{Comparison Tables for Pladienolides A (1) and B (2)}

Table S8. Comparison of ${ }^{1} \mathrm{H}$ NMR spectroscopic data of natural and synthetic pladienolide A (1)

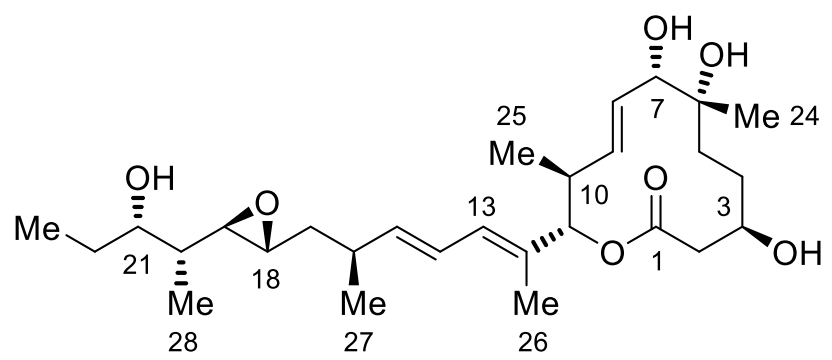

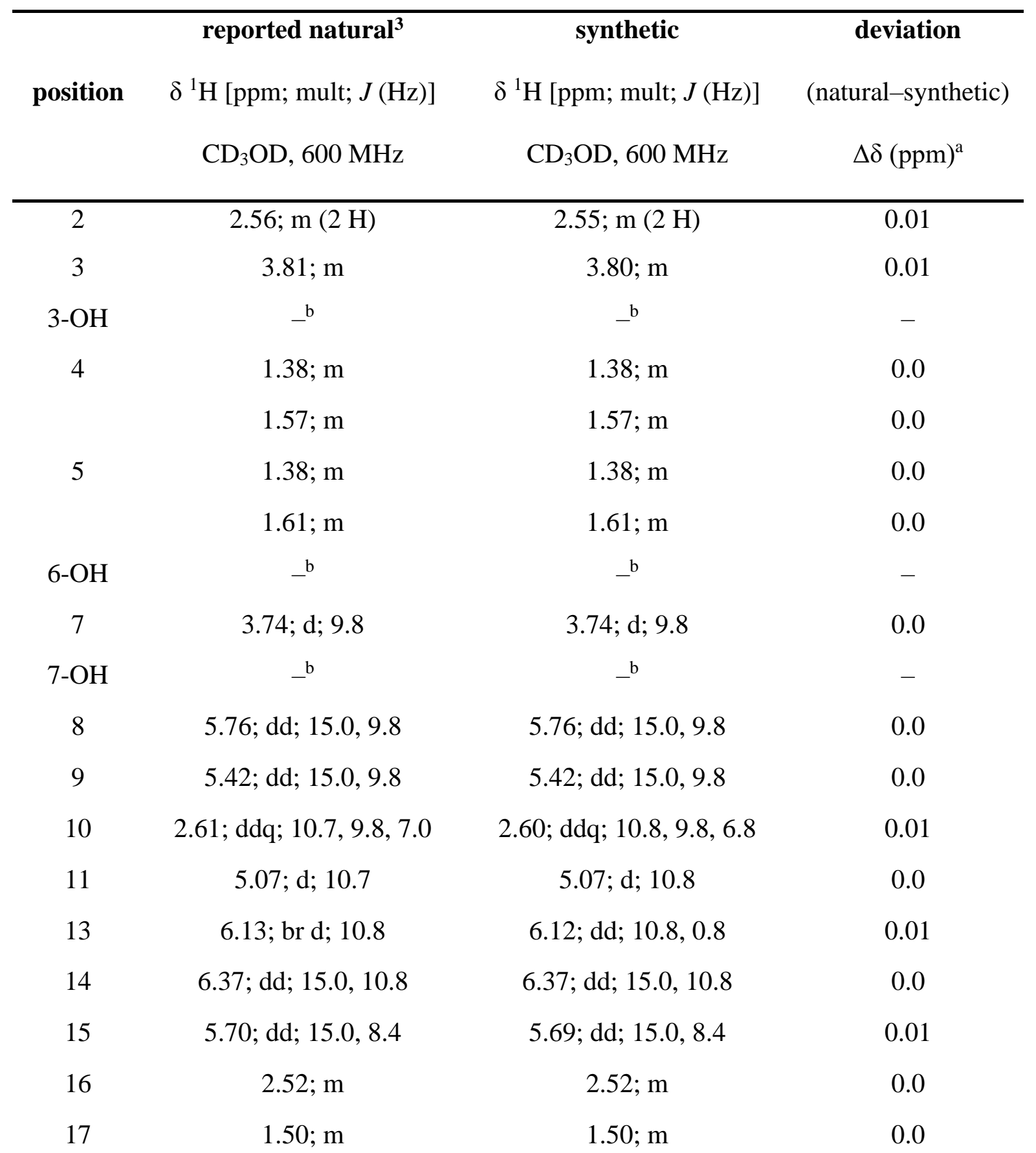




\begin{tabular}{cccc} 
& \multicolumn{3}{c}{ D. Rhoades, * et al. } \\
& $1.68 ;$ ddd; $14.0,5.9,5.5$ & $1.68 ;$ ddd; $13.8,5.6,5.5$ & 0.0 \\
18 & $2.77 ;$ ddd; $5.9,2.2,2.2$ & $2.77 ;$ ddd; $5.9,2.2,2.2$ & 0.0 \\
19 & $2.70 ; \mathrm{dd} ; 8.2,2.2$ & $2.70 ; \mathrm{dd} ; 8.2,2.2$ & 0.0 \\
20 & $1.24 ; \mathrm{m}$ & $1.24 ; \mathrm{dqd} ; 8.2,6.7,4.5$ & 0.0 \\
21 & $3.56 ; \mathrm{ddd} ; 8.7,4.5,4.5$ & $3.56 ; \mathrm{ddd} ; 8.7,4.5,4.5$ & 0.0 \\
$21-\mathrm{OH}$ & $-{ }^{\mathrm{b}}$ & $-{ }^{\mathrm{b}}$ & - \\
22 & $1.56 ; \mathrm{m}(2 \mathrm{H})$ & $1.56 ;(\mathrm{m} 2 \mathrm{H})$ & 0.0 \\
23 & $0.98 ; \mathrm{t} ; 7.4$ & $0.98 ; \mathrm{app} \mathrm{t} ; 7.4$ & 0.0 \\
24 & $1.31 ; \mathrm{s}$ & $1.31 ; \mathrm{s}$ & 0.0 \\
25 & $0.95 ; \mathrm{d} ; 6.7$ & $0.948 ; \mathrm{d} ; 6.8$ & 0.002 \\
26 & $1.79 ; \mathrm{br} \mathrm{s}$ & $1.79 ; \mathrm{d} ; 0.8$ & 0.0 \\
27 & $1.13 ; \mathrm{d} ; 6.8$ & $1.12 ; \mathrm{d} ; 6.8$ & 0.01 \\
28 & $0.95 ; \mathrm{d} ; 7.0$ & $0.945 ; \mathrm{d} ; 7.0$ & 0.005
\end{tabular}

${ }^{a}$ Chemical shift values $(\delta, \mathrm{ppm})$ for synthetic $\mathbf{1}$ are based on the reference value used by Y. Kotake et al. (3.35 ppm). ${ }^{3}$ ${ }^{b}$ Due to solvent exchange with $\mathrm{CD}_{3} \mathrm{OD}$, hydroxy groups were not observable. 
D. Rhoades, * et al.

Table S9. Comparison of ${ }^{13} \mathrm{C}$ NMR spectroscopic data of natural and synthetic pladienolide A (1)

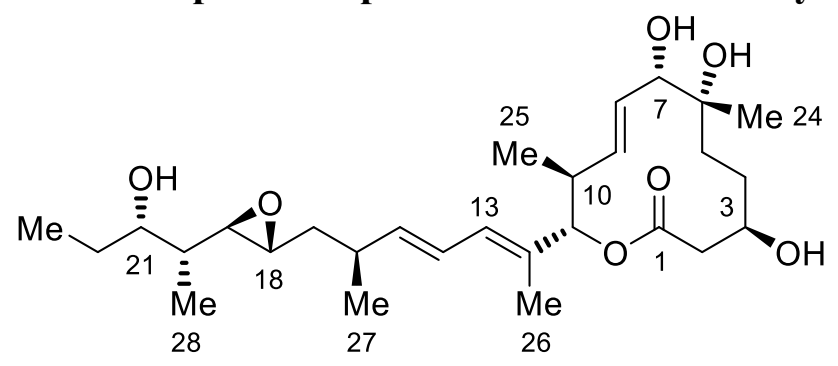

\begin{tabular}{|c|c|c|c|}
\hline position & $\begin{array}{c}\text { reported natural }^{3} \\
\delta{ }^{13} \mathrm{C}[\mathrm{ppm}]\end{array}$ & $\begin{array}{c}\text { synthetic } \\
\delta{ }^{13} \mathrm{C}[\mathrm{ppm}]\end{array}$ & $\begin{array}{c}\text { deviation } \\
\text { (natural-synthetic) }\end{array}$ \\
\hline & $\mathrm{CD}_{3} \mathrm{OD}, 151 \mathrm{MHz}$ & $\mathrm{CD}_{3} \mathrm{OD}, 151 \mathrm{MHz}$ & $\Delta \delta(\mathrm{ppm})^{\mathrm{a}}$ \\
\hline 1 & 171.9 & 171.9 & 0.0 \\
\hline 2 & 40.1 & 40.1 & 0.0 \\
\hline 3 & 70.7 & 70.7 & 0.0 \\
\hline 4 & 30.5 & 30.5 & 0.0 \\
\hline 5 & 37.6 & 37.6 & 0.0 \\
\hline 6 & 74.7 & 74.7 & 0.0 \\
\hline 7 & 78.2 & 78.2 & 0.0 \\
\hline 8 & 131.6 & 131.6 & 0.0 \\
\hline 9 & 137.7 & 137.7 & 0.0 \\
\hline 10 & 41.8 & 41.8 & 0.0 \\
\hline 11 & 84.4 & 84.4 & 0.0 \\
\hline 12 & 132.1 & 132.1 & 0.0 \\
\hline 13 & 132.6 & 132.6 & 0.0 \\
\hline 14 & 125.9 & 125.9 & 0.0 \\
\hline 15 & 142.3 & 142.3 & 0.0 \\
\hline 16 & 36.7 & 36.8 & -0.1 \\
\hline 17 & 40.7 & 40.7 & 0.0 \\
\hline 18 & 58.5 & 58.5 & 0.0 \\
\hline 19 & 63.0 & 63.0 & 0.0 \\
\hline 20 & 42.8 & 42.8 & 0.0 \\
\hline
\end{tabular}


D. Rhoades, ${ }^{*}$ et al.

\begin{tabular}{lccc}
21 & 75.3 & 75.3 & 0.0 \\
22 & 28.6 & 28.6 & 0.0 \\
23 & 10.8 & 10.86 & -0.06 \\
24 & 24.4 & 24.4 & 0.0 \\
25 & 17.1 & 17.1 & 0.0 \\
26 & 11.9 & 11.9 & 0.0 \\
27 & 21.7 & 21.7 & 0.0 \\
28 & 10.9 & 10.88 & 0.02 \\
\hline
\end{tabular}

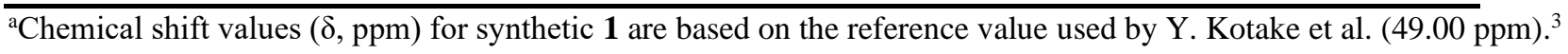


Table S10. Comparison of ${ }^{1} \mathrm{H}$ NMR spectroscopic data of natural and synthetic pladienolide B (2)

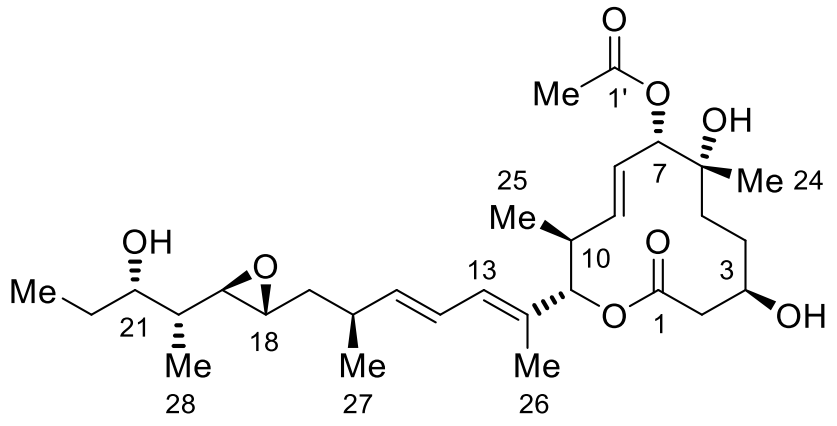

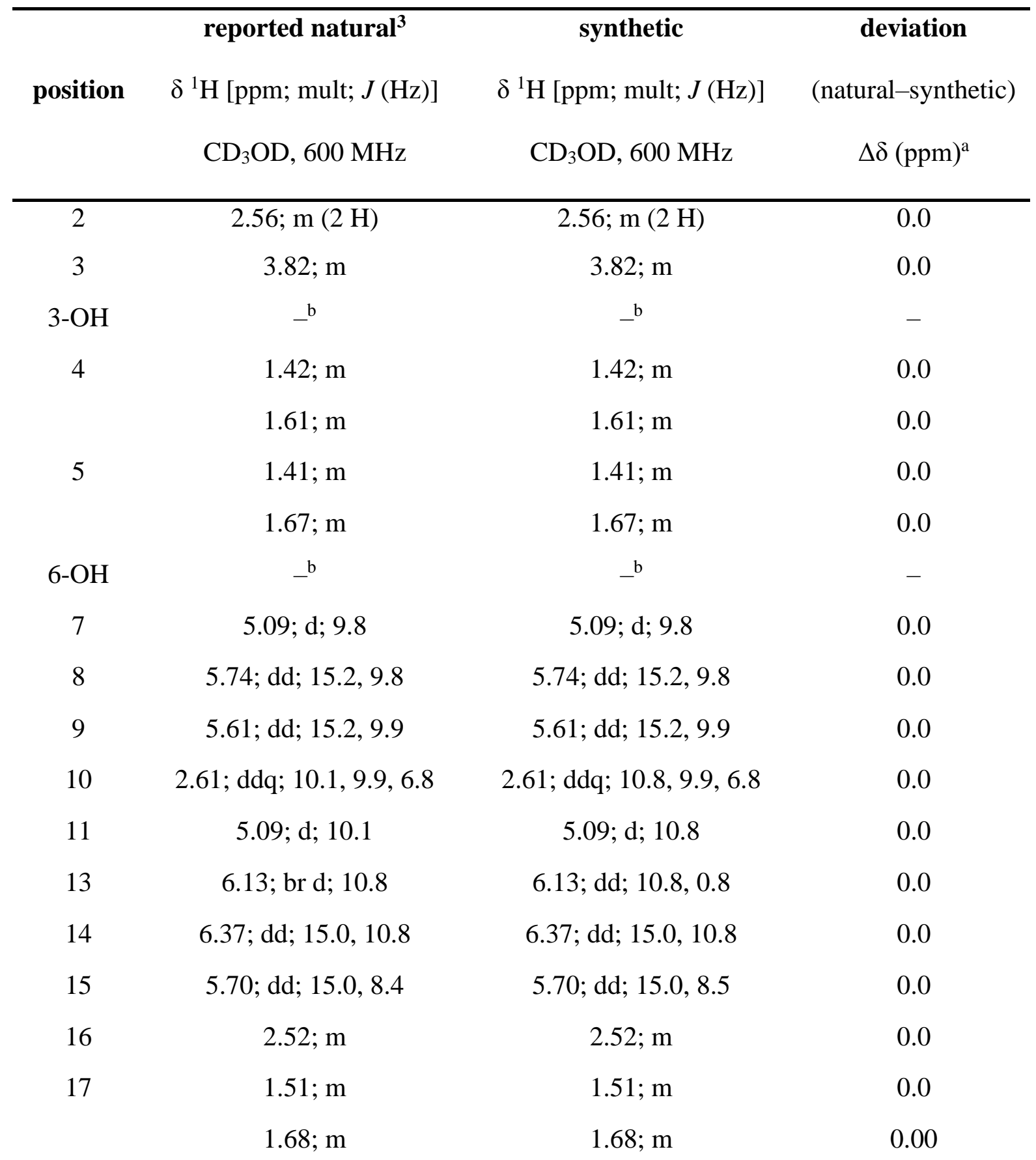




\begin{tabular}{cccc} 
& \multicolumn{3}{c}{ D. Rhoades, et al. } \\
18 & $2.77 ; \mathrm{ddd} ; 5.9,5.9,2.2$ & $2.76 ; \mathrm{ddd} ; 6.0,5.5,2.2$ & 0.01 \\
19 & $2.70 ; \mathrm{dd} ; 8.2,2.2$ & $2.70 ; \mathrm{dd} ; 8.2,2.2$ & 0.0 \\
20 & $1.24 ; \mathrm{m}$ & $1.24 ; \mathrm{m}$ & 0.0 \\
21 & $3.55 ; \mathrm{ddd} ; 8.7,4.5,4.5$ & $3.55 ; \mathrm{ddd} ; 8.7,4.5,4.5$ & 0.0 \\
$21-\mathrm{OH}$ & $-{ }^{\mathrm{b}}$ & $-{ }^{\mathrm{b}}$ & - \\
22 & $1.52 ; \mathrm{m}(2 \mathrm{H})$ & $1.52 ; \mathrm{m}(2 \mathrm{H})$ & 0.0 \\
23 & $0.98 ; \mathrm{t} ; 7.4$ & $0.98 ; \mathrm{app} \mathrm{t} ; 7.4$ & 0.0 \\
24 & $1.23 ; \mathrm{s}$ & $1.23 ; \mathrm{br} \mathrm{s}$ & 0.0 \\
25 & $0.93 ; \mathrm{d} ; 6.8$ & $0.92 ; \mathrm{d} ; 6.8$ & 0.01 \\
26 & $1.79 ; \mathrm{br} \mathrm{s}$ & $1.79 ; \mathrm{d} ; 0.8$ & 0.0 \\
27 & $1.13 ; \mathrm{d} ; 6.8$ & $1.12 ; \mathrm{d} ; 6.8$ & 0.01 \\
28 & $0.95 ; \mathrm{d} ; 7.0$ & $0.94 ; \mathrm{d} ; 7.0$ & 0.01 \\
2 & $2.10 ; \mathrm{s}$ & $2.10 ; \mathrm{s}$ & 0.0
\end{tabular}

${ }^{\mathrm{a} C h e m i c a l}$ shift values $(\delta, \mathrm{ppm})$ for synthetic $\mathbf{2}$ are based on the reference value used by Y. Kotake et al. (3.35 ppm). ${ }^{3}$ ${ }^{b}$ Due to solvent exchange with $\mathrm{CD}_{3} \mathrm{OD}$, hydroxy groups were not observable. 


\section{Rhoades, * et al.}

Table S11. Comparison of ${ }^{13}$ C NMR spectroscopic data of natural and synthetic pladienolide B (2)

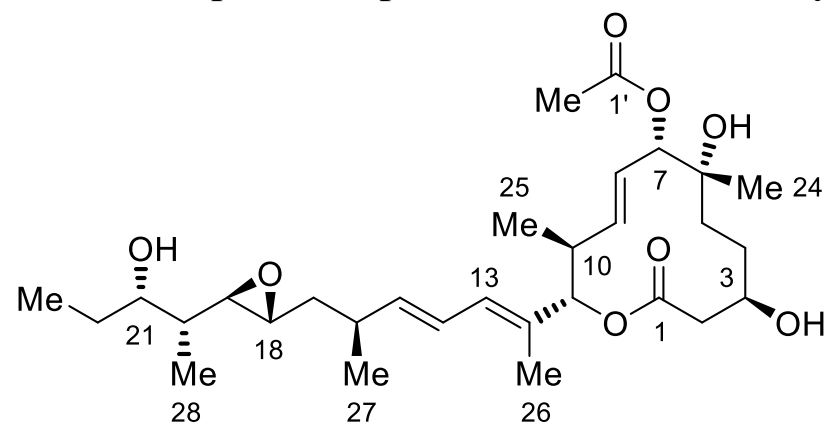

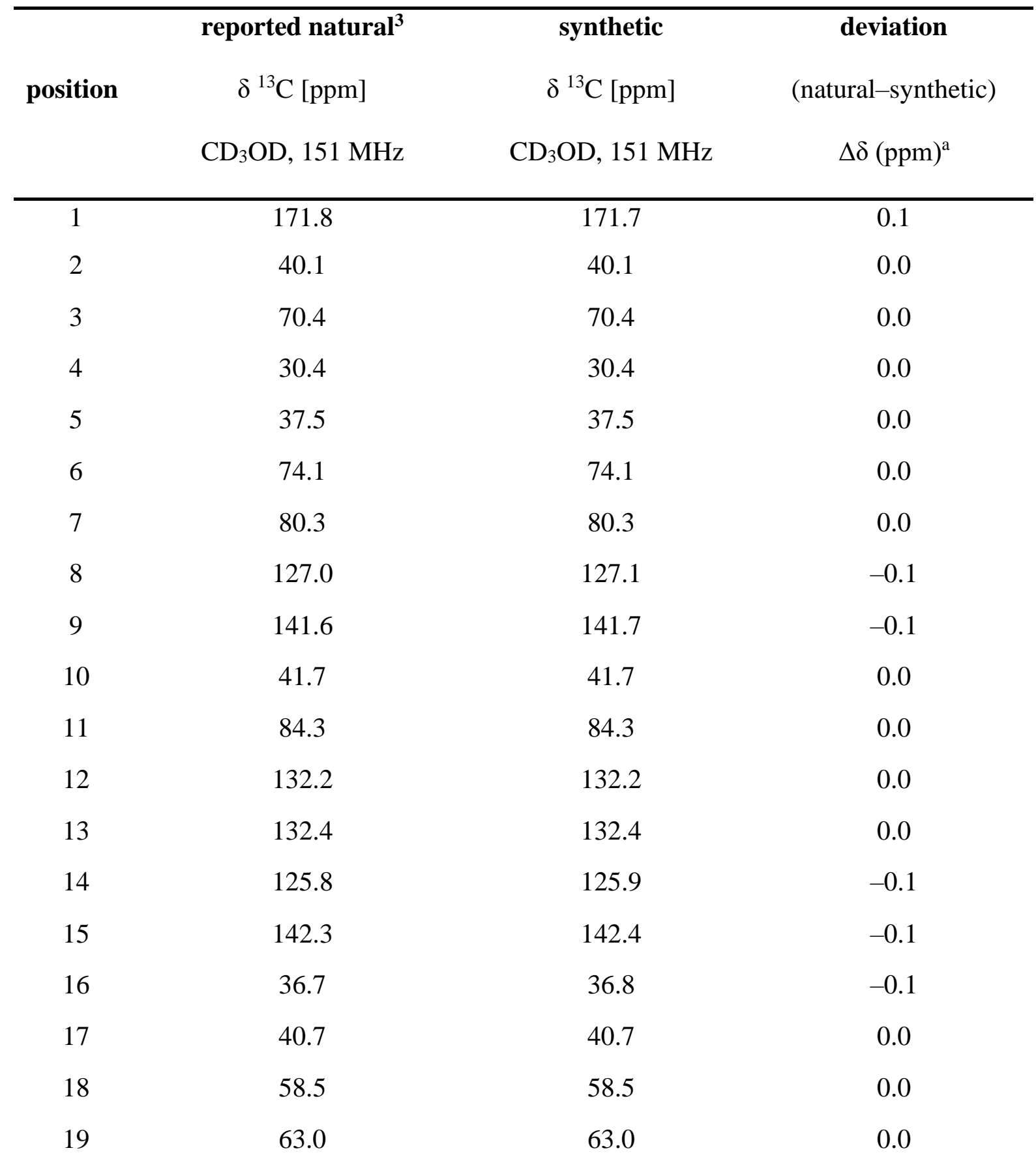




\begin{tabular}{cccc} 
& & D. Rhoades, ${ }^{*}$ et al. & \\
20 & 42.8 & 42.8 & 0.0 \\
21 & 75.3 & 75.3 & 0.0 \\
22 & 28.6 & 28.6 & 0.0 \\
23 & 10.8 & 10.86 & -0.06 \\
24 & 24.2 & 24.2 & 0.0 \\
25 & 16.9 & 16.9 & 0.0 \\
26 & 11.9 & 11.9 & 0.0 \\
27 & 21.7 & 21.7 & 0.0 \\
28 & 10.9 & 10.88 & 0.02 \\
$1^{\prime}$ & 172.2 & 172.2 & 0.0 \\
$2^{\prime}$ & 21.1 & 21.1 & 0.0 \\
\hline${ }^{a}$ Chemical shift values $\left(\delta\right.$, ppm) for synthetic 2 are based on the reference value used by Y. Kotake et al. $(49.00 \text { ppm })^{3}$
\end{tabular}




\section{Rhoades, * et al.}

\section{NMR Spectroscopic Assignments for H3B-8800 (3)}

Table S12. ${ }^{1} \mathrm{H}$ and ${ }^{13} \mathrm{C}$ NMR spectroscopic assignments for H3B-8800 (3)

\begin{tabular}{|c|c|c|c|}
\hline \multirow{3}{*}{ position } & semisynthetic $^{7}$ & synthetic & synthetic \\
\hline & $\delta{ }^{1} \mathrm{H}[\mathrm{ppm} ; \text { mult; } J(\mathrm{~Hz})]^{\mathrm{a}}$ & $\delta{ }^{1} \mathrm{H}[\mathrm{ppm} ; \text { mult; } J(\mathrm{~Hz})]^{\mathrm{b}}$ & $\delta^{13} \mathrm{C}(\mathrm{ppm})^{\mathrm{b}}$ \\
\hline & $\mathrm{CDCl}_{3}, 400 \mathrm{MHz}$ & $\mathrm{CDCl}_{3}, 600 \mathrm{MHz}$ & $\mathrm{CDCl}_{3}, 151 \mathrm{MHz}$ \\
\hline 1 & - & - & 172.2 \\
\hline \multirow[t]{2}{*}{2} & $2.45-2.68 ; \mathrm{m}$ & $2.53 ; \mathrm{dd} ; 14.9,2.7$ & 38.5 \\
\hline & $2.45-2.68 ; \mathrm{m}$ & $2.61 ; \mathrm{dd} ; 14.9,3.5$ & \\
\hline 3 & 3.74; dd; 14.2. 7.2 & $3.74 ; \mathrm{m}$ & 69.4 \\
\hline $3-\mathrm{OH}$ & 2.07; s & 1.54 ; br s & - \\
\hline \multirow[t]{2}{*}{4} & $1.22-1.48 ; \mathrm{m}$ & $1.31 ;$ app tt; $13.3,2.7$ & 30.0 \\
\hline & $1.66-1.83 ; \mathrm{m}$ & 1.70; app tt; 13.3, 3.8 & - \\
\hline \multirow[t]{2}{*}{5} & $1.22-1.48 ; \mathrm{m}$ & 1.37; app td; $14.0,3.4$ & 35.5 \\
\hline & $1.50-1.63 ; \mathrm{m}$ & 1.53; app td; 14.0, 3.2 & - \\
\hline 6 & - & - & 74.0 \\
\hline $6-\mathrm{OH}$ & 1.97; s & 1.94; br s & - \\
\hline 7 & $5.04 ; \mathrm{d} ; 9.3$ & $5.01 ; \mathrm{d} ; 9.7$ & 80.2 \\
\hline 8 & $5.57-5.76 ; \mathrm{m}$ & $5.68 ; \mathrm{dd} ; 15.2,9.7$ & 126.1 \\
\hline 9 & $5.57-5.76 ; \mathrm{m}$ & $5.60 ; \mathrm{dd} ; 15.2,9.8$ & 140.2 \\
\hline 10 & $2.45-2.68 ; \mathrm{m}$ & $2.51 ; \mathrm{ddq} ; 10.7,9.8,6.8$ & 40.9 \\
\hline 11 & $5.17 ; \mathrm{d} ; 10.5$ & $5.15 ; \mathrm{d} ; 10.7$ & 82.9 \\
\hline $12^{\mathrm{c}}$ & - & - & 131.35 \\
\hline $13^{\mathrm{c}}$ & $6.13 ; \mathrm{d} ; 10.8$ & $6.11 ;$ br d; 10.8 & 131.31 \\
\hline
\end{tabular}


D. Rhoades, * et al.

$\begin{array}{lccc}14 & 6.34 ; \text { ddd; } 15.1,10.7,1.0 & 6.32 ; \mathrm{ddd} ; 15.1,10.8,1.0 & 124.8 \\ 15 & 6.02 ; \mathrm{dd} ; 15.1,7.5 & 6.00 ; \mathrm{dd} ; 15.1,7.5 & 139.9 \\ 16 & 3.74 ; \mathrm{dd} ; 14.2 .7 .2 & 3.71 ; \mathrm{dqd} ; 7.5,7.0,1.0 & 45.5 \\ 17 & - & - & 164.6 \\ 18 & 7.18 ; \mathrm{d} ; 7.4 & 7.16 ; \mathrm{ddd} ; 7.9,1.2,0.9 & 121.7 \\ 19 & 7.63 ; \mathrm{t} ; 7.3 & 7.61 ; \mathrm{ddd} ; 7.9,7.5,1.9 & 136.8 \\ 20 & 7.14 ; \mathrm{t} ; 6.2 & 7.12 ; \mathrm{ddd} ; 7.5,4.8,1.2 & 121.6 \\ 21 & 8.57 ; \mathrm{d} ; 5.1 & 8.55 ; \mathrm{ddd} ; 4.8,1.9,0.9 & 149.5 \\ 22 & 1.22-1.48 ; \mathrm{m} & 1.23 ; \mathrm{br} \mathrm{s} & 24.9 \\ 23 & 0.82-1.00 ; \mathrm{m} & 0.88 ; \mathrm{d} ; 6.8 & 16.7 \\ 24 & 1.66-1.83 ; \mathrm{m} & 1.72 ; \mathrm{br} \mathrm{s} & 11.9 \\ 25 & 1.22-1.48 ; \mathrm{m} & 1.44 ; \mathrm{d} ; 7.0 & 20.5 \\ 1^{\prime} & - & 2.37 ; \mathrm{m} ;(4 \mathrm{H}) & 154.5 \\ 2^{\prime} & 2.43 ; \mathrm{br} \mathrm{s} & 2.30 ; \mathrm{br} \mathrm{s} & 44.1 \\ 3^{\prime} & 3.44-3.61 ; \mathrm{m} & 3.50 ; \mathrm{m}(4 \mathrm{H}) & 54.9 \\ 4^{\prime} & 2.33 & 46.3\end{array}$

${ }^{\mathrm{a} O n l y}{ }^{1} \mathrm{H}$ NMR chemical shift values $(\delta, \mathrm{ppm})$ were reported for semisynthetic $3{ }^{7}$

${ }^{\mathrm{b}}$ Chemical shift values for synthetic 3 are based on accepted reference values $\left(\delta_{\mathrm{H}}=7.26 \mathrm{ppm} ; \delta_{\mathrm{C}}=77.16 \mathrm{ppm}\right){ }^{2}$

${ }^{\mathrm{c}} \mathrm{C} 12$ vs. $\mathrm{C} 13$ identification was unambiguously determined by ${ }^{13} \mathrm{C}$ DEPTQ135 and DEPT90 NMR (vide infra). 
D. Rhoades, * et al.

VIII. ${ }^{1} \mathrm{H},{ }^{13} \mathrm{C},{ }^{19} \mathrm{~F}$, and Selected 2D NMR Spectra of Compounds

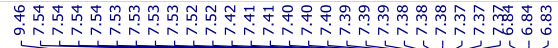

$\mathrm{PhMe}_{2} \mathrm{Si} \overbrace{\mathrm{Me}}$

6

${ }^{1} \mathrm{H}$ NMR spectrum

$\left(\mathrm{CDCl}_{3}, 600 \mathrm{MHz}\right)$
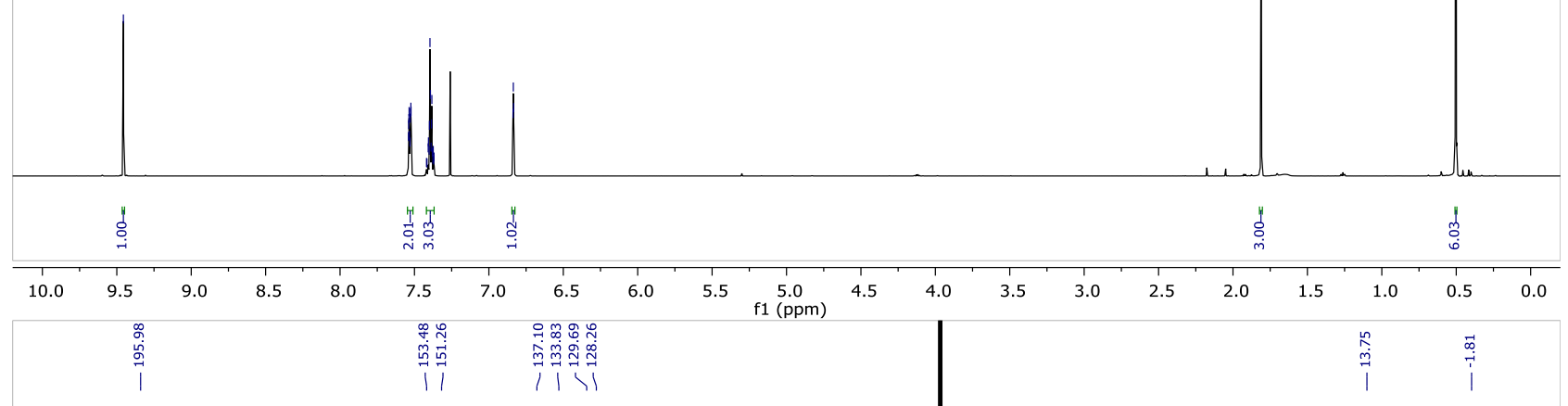

$\mathrm{PhMe}_{2} \mathrm{Si} \overbrace{\mathrm{Me}} \mathrm{O}_{\mathrm{O}}$

6

${ }^{13} \mathrm{C}$ NMR spectrum

$\left(\mathrm{CDCl}_{3}, 151 \mathrm{MHz}\right)$

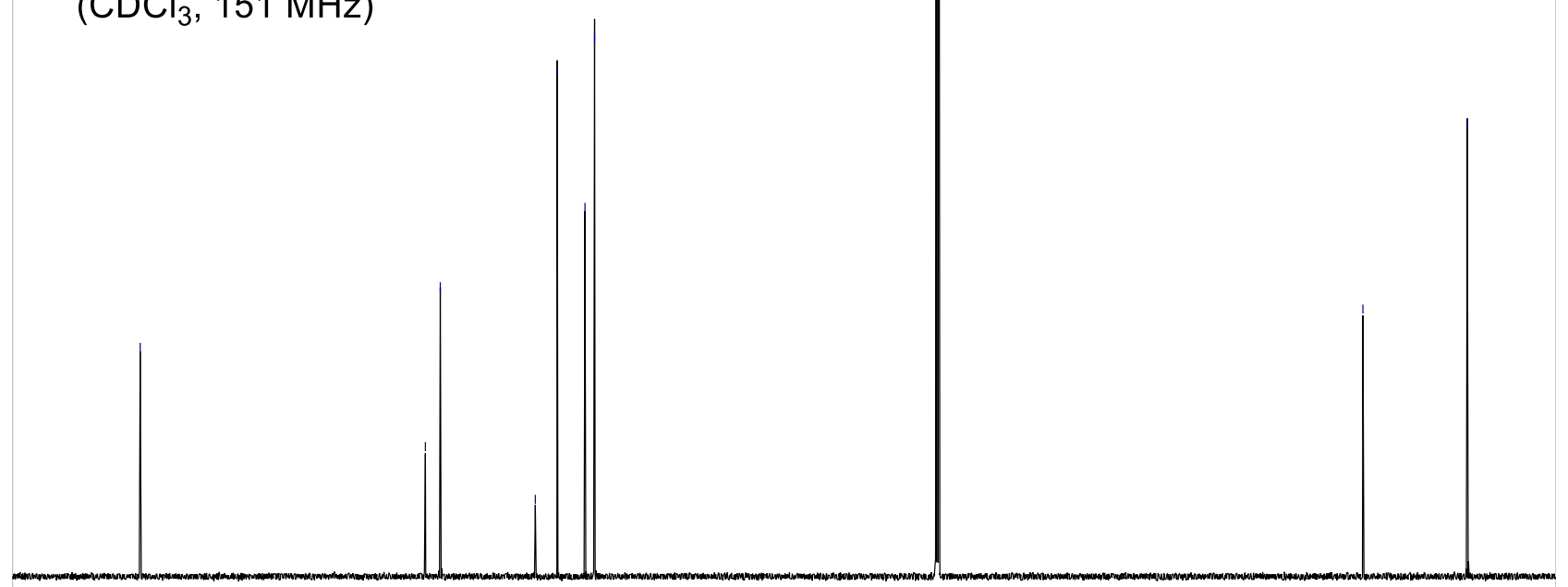

$\begin{array}{lllllllllllllllllllllllll}210 & 200 & 190 & 180 & 170 & 160 & 150 & 140 & 130 & 120 & 110 & 100 & 90 & 80 & 70 & 60 & 50 & 40 & 30 & 20 & 10 & 0 & -10\end{array}$ 
D. Rhoades, * et al.

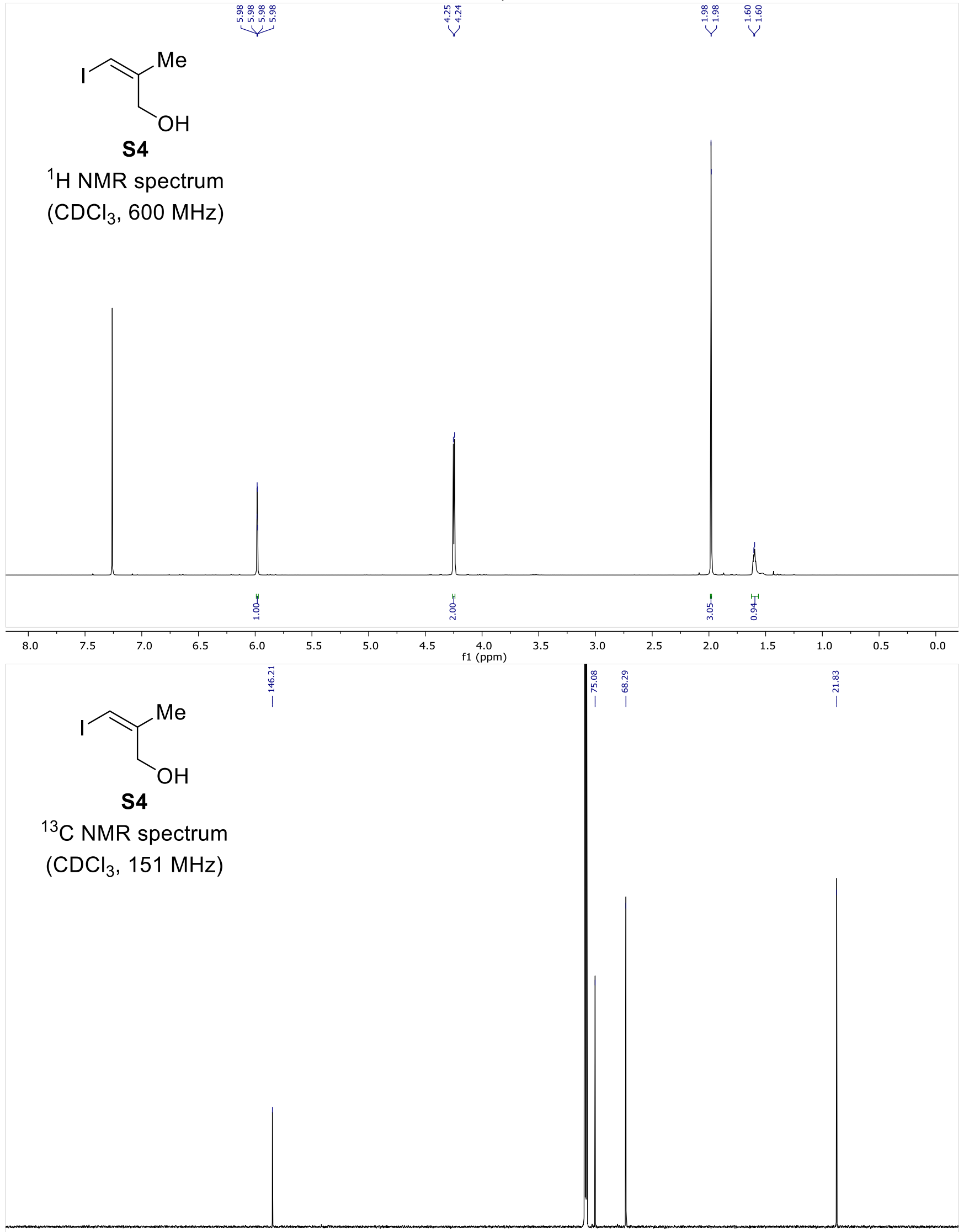

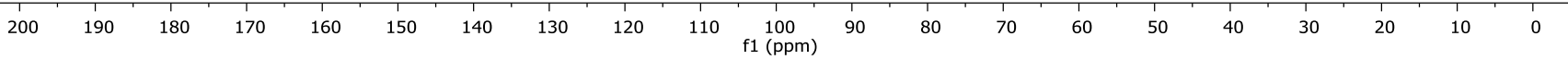
S76 
D. Rhoades, * et al.

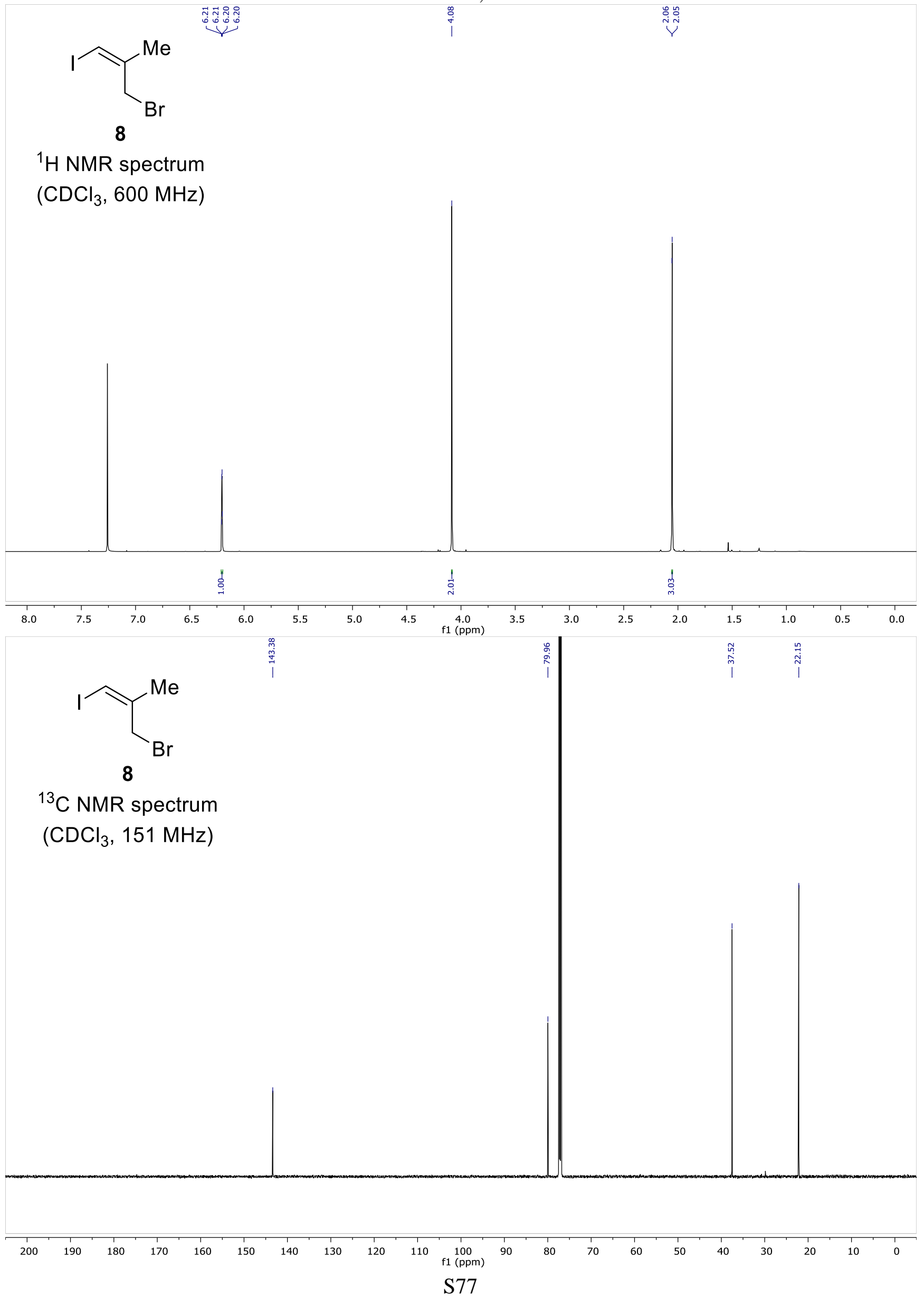


D. Rhoades, * et al.

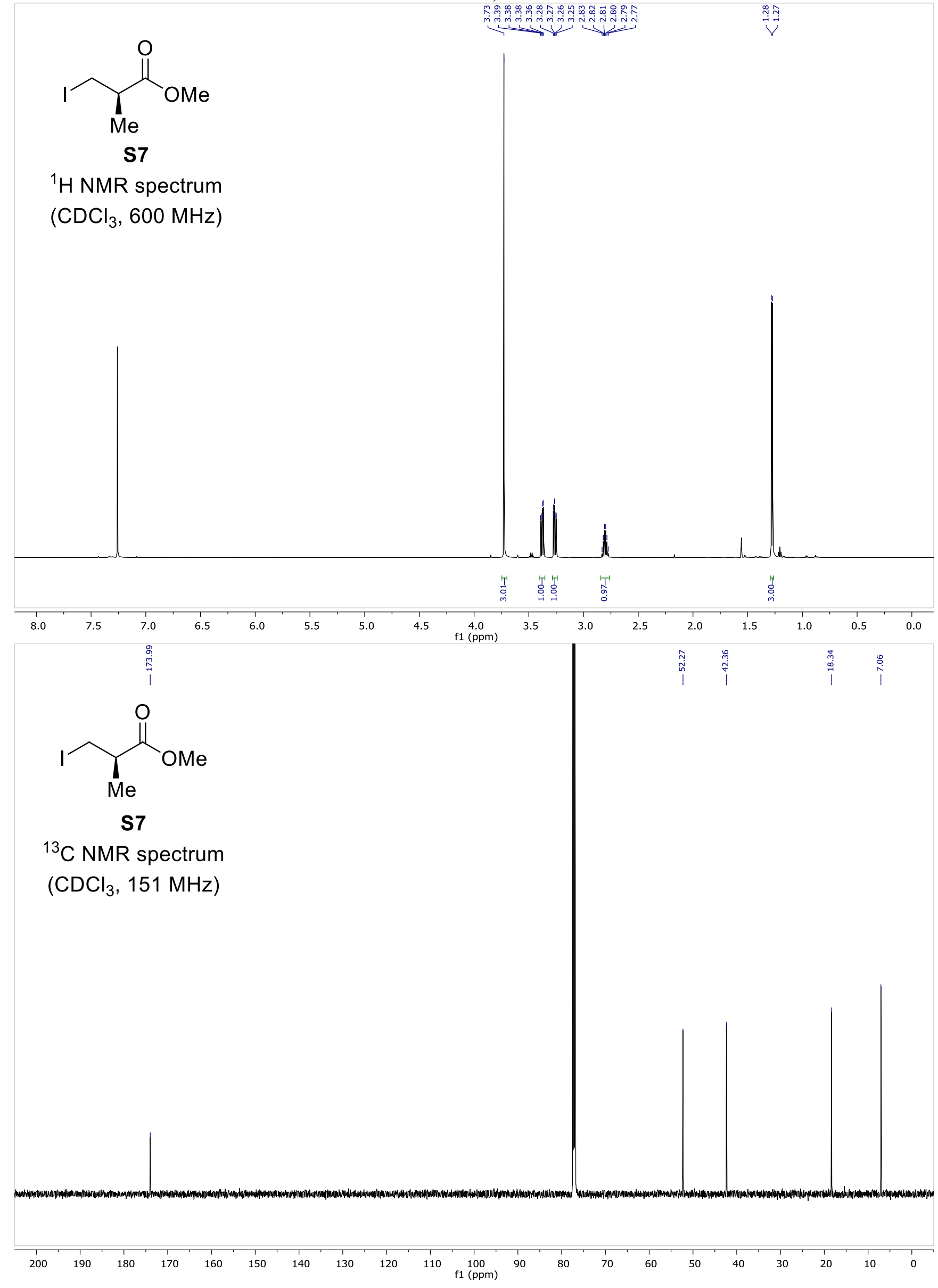


D. Rhoades, * et al.

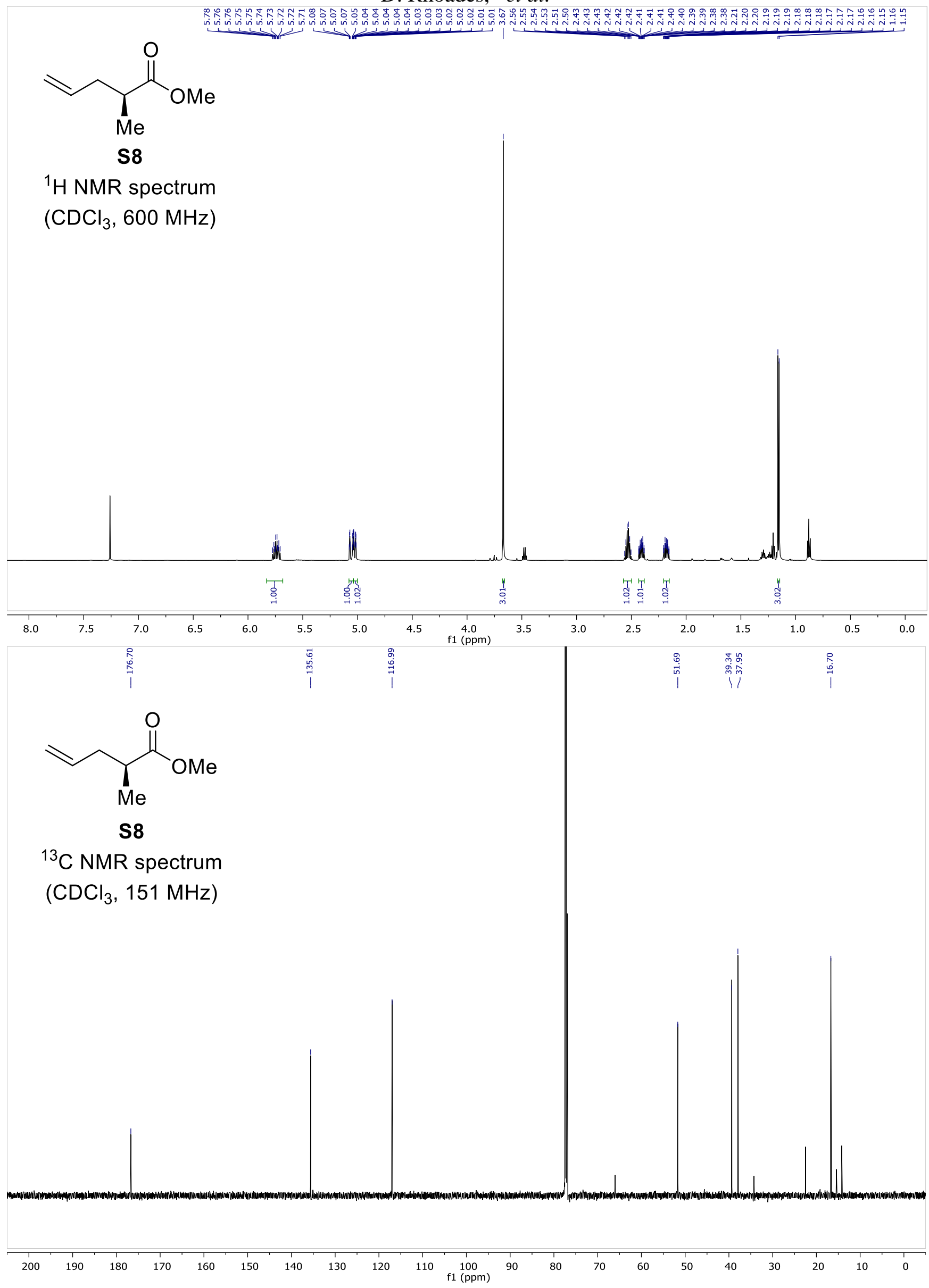


D. Rhoades, * et al.

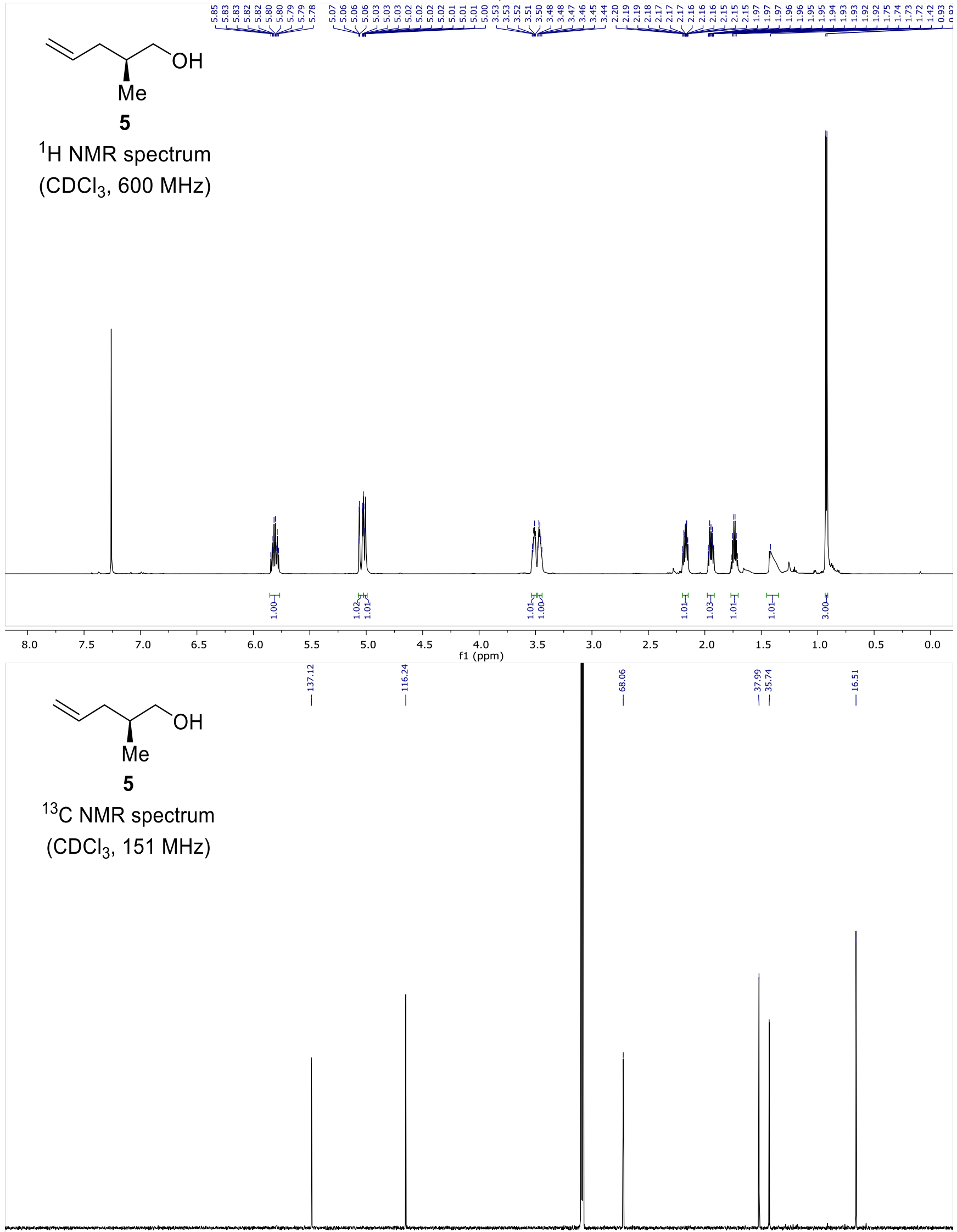


D. Rhoades, * et al.<smiles>C=C[C@H](C)[C@@H](O)CC</smiles>

4

${ }^{1} \mathrm{H}$ NMR spectrum

$\left(\mathrm{CDCl}_{3}, 600 \mathrm{MHz}\right)$

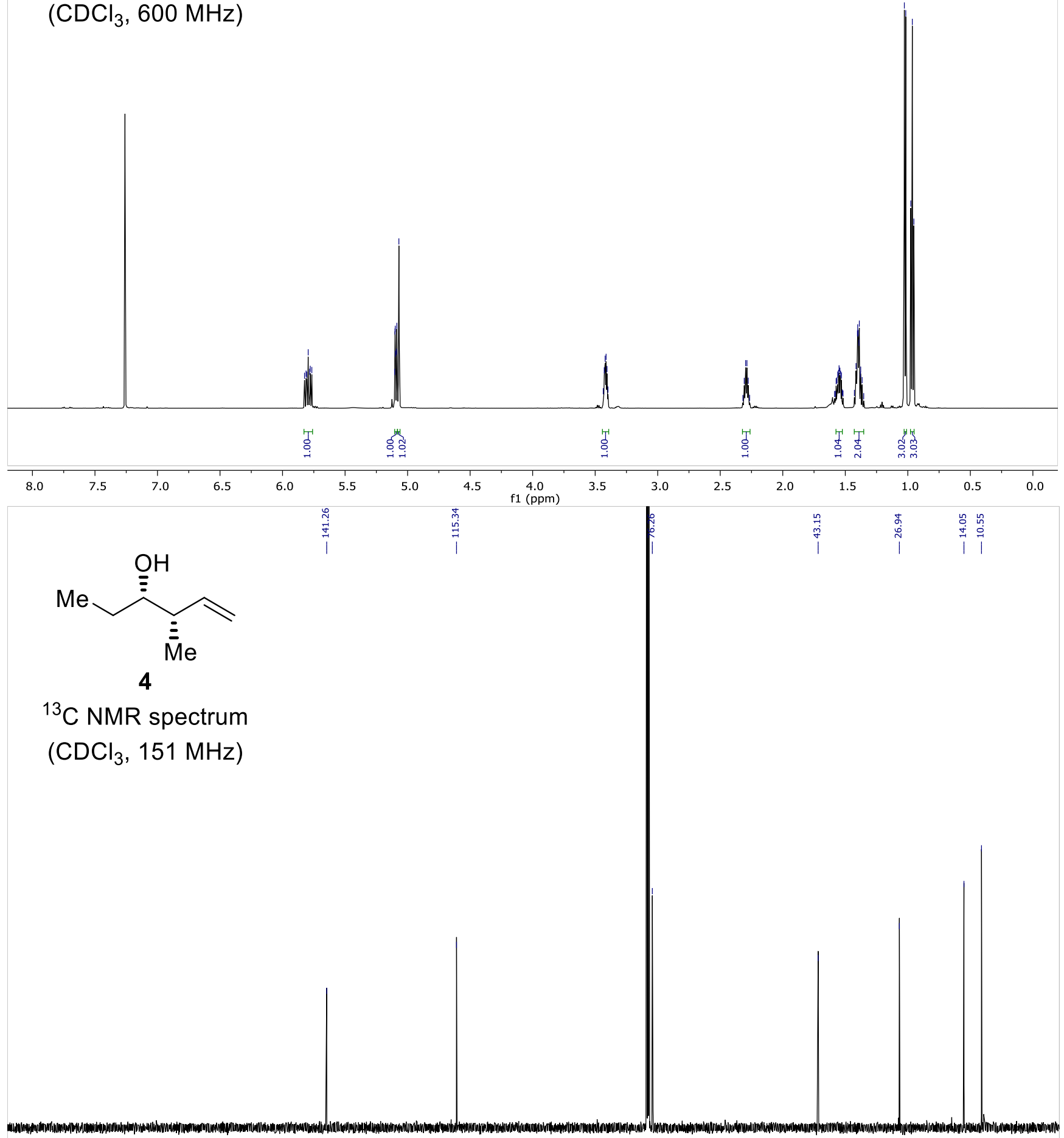


D. Rhoades, * et al.

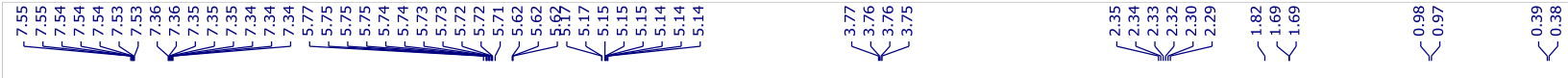<smiles>C=C[C@H](C)[C@H](O)/C(C)=C/[SiH2]C(C)C</smiles>

12

${ }^{1} \mathrm{H}$ NMR spectrum $\left(\mathrm{CDCl}_{3}, 600 \mathrm{MHz}\right)$
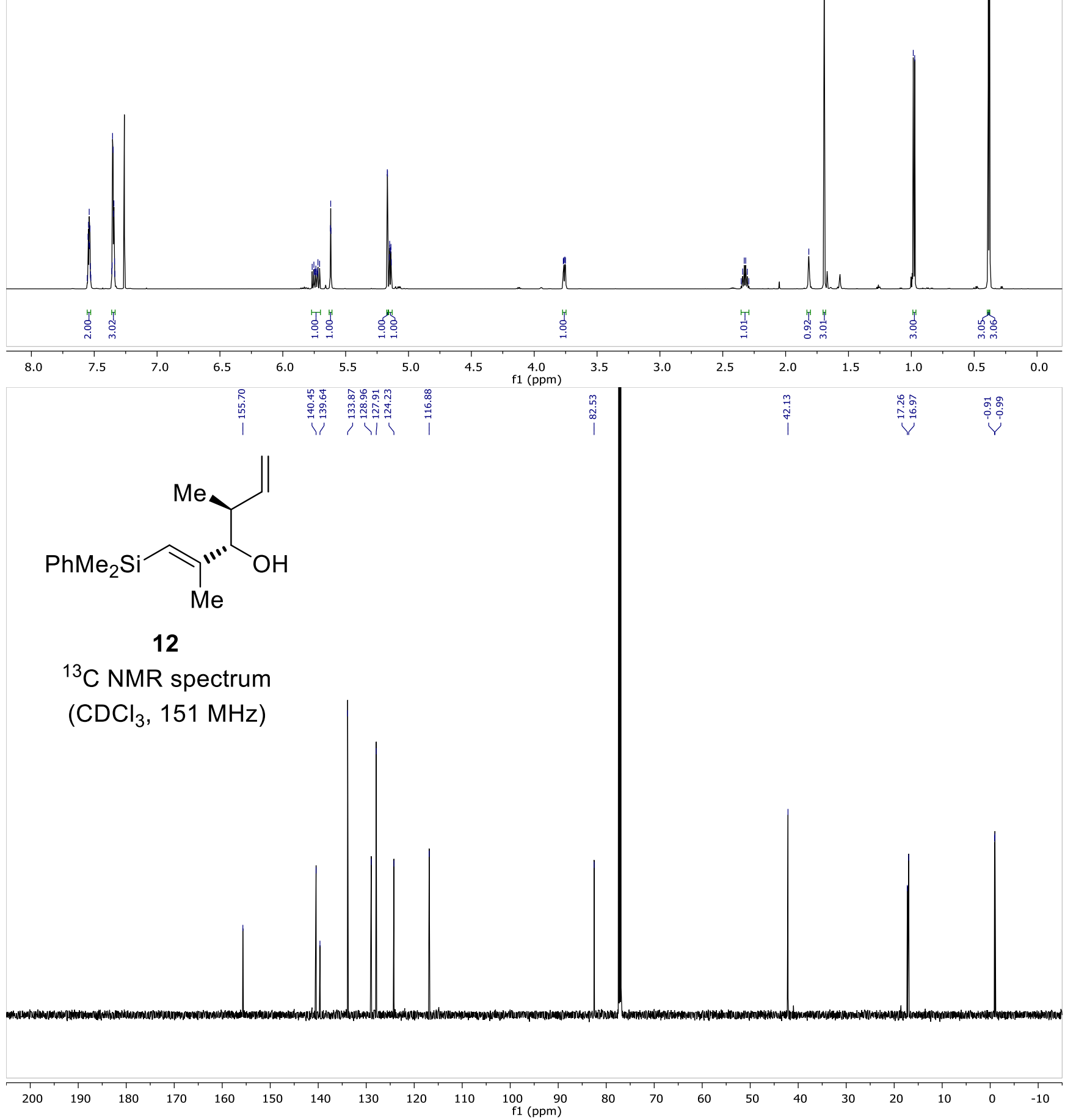
D. Rhoades, * et al.

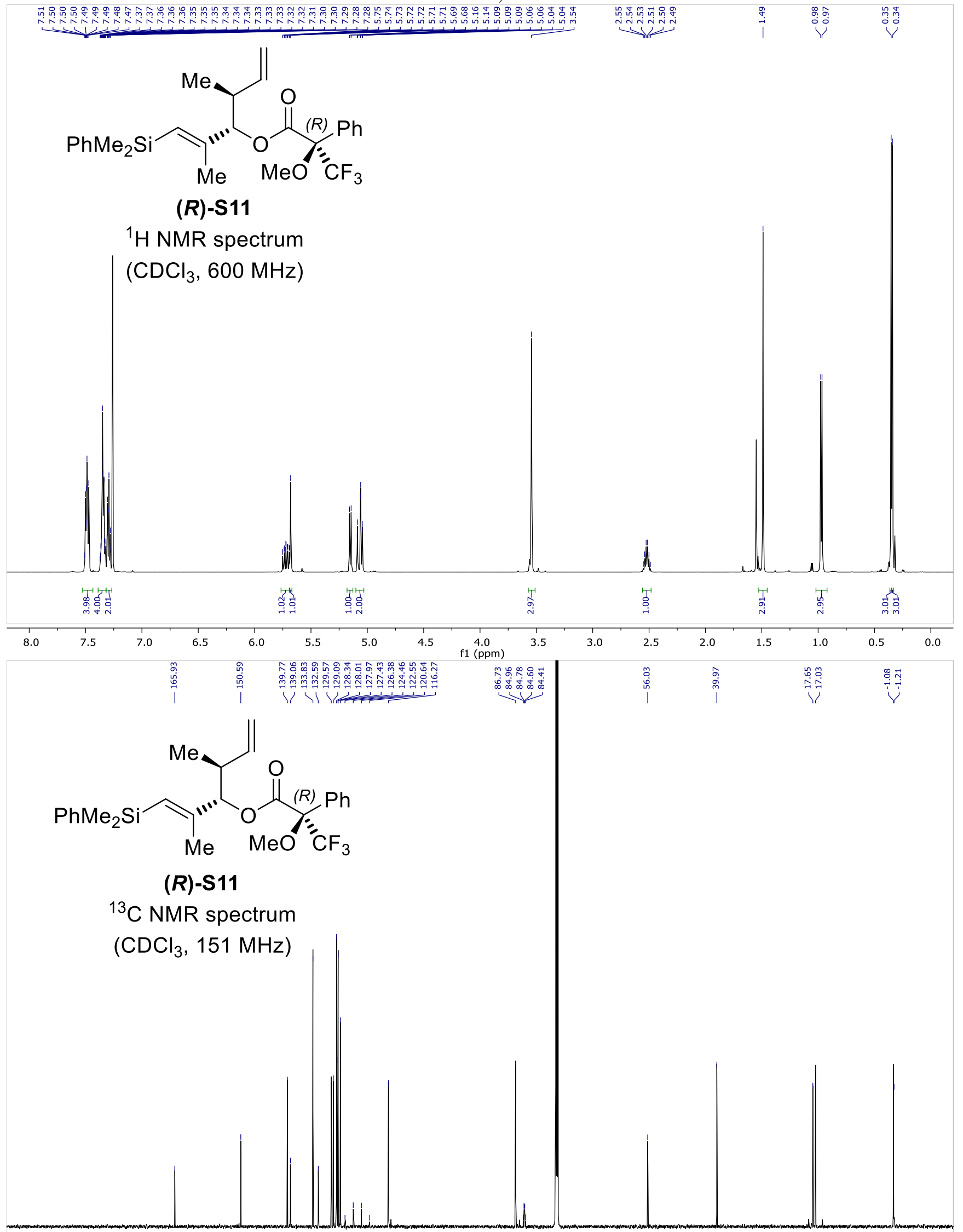

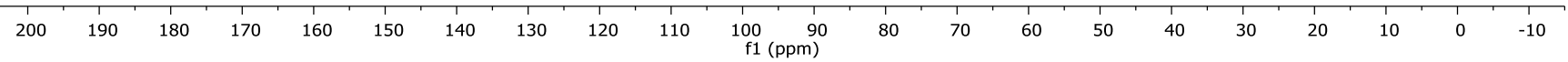


D. Rhoades, * et al.

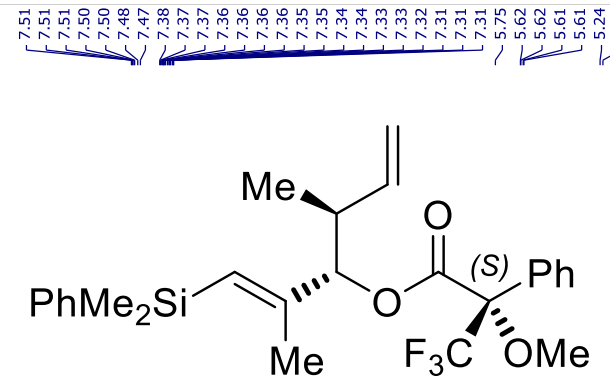

(S)-S11

${ }^{1} \mathrm{H}$ NMR spectrum

$\left(\mathrm{CDCl}_{3}, 600 \mathrm{MHz}\right)$

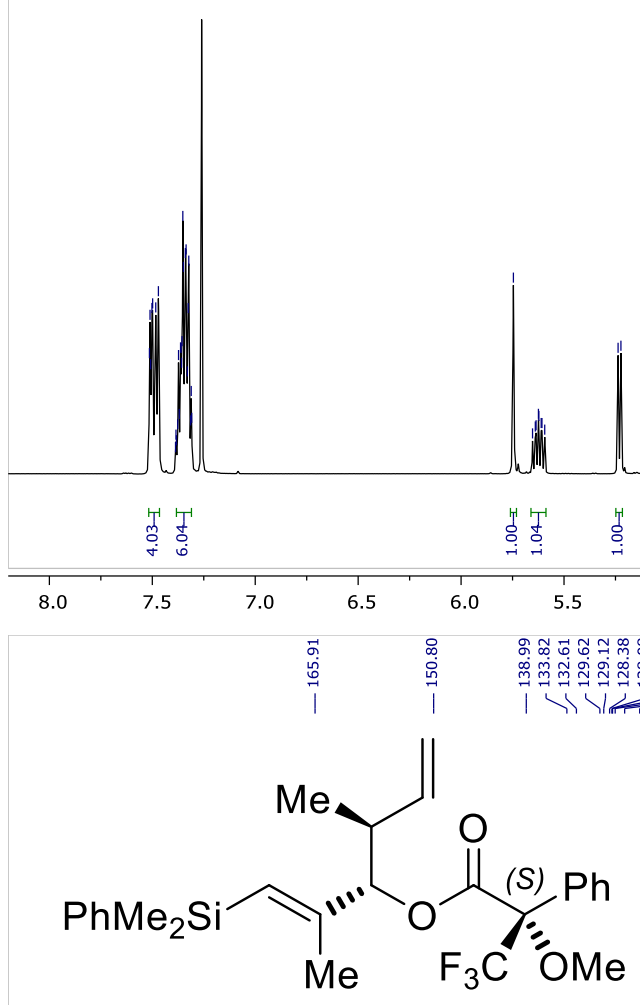

(S)-S11

${ }^{13} \mathrm{C}$ NMR spectrum

$\left(\mathrm{CDCl}_{3}, 151 \mathrm{MHz}\right)$

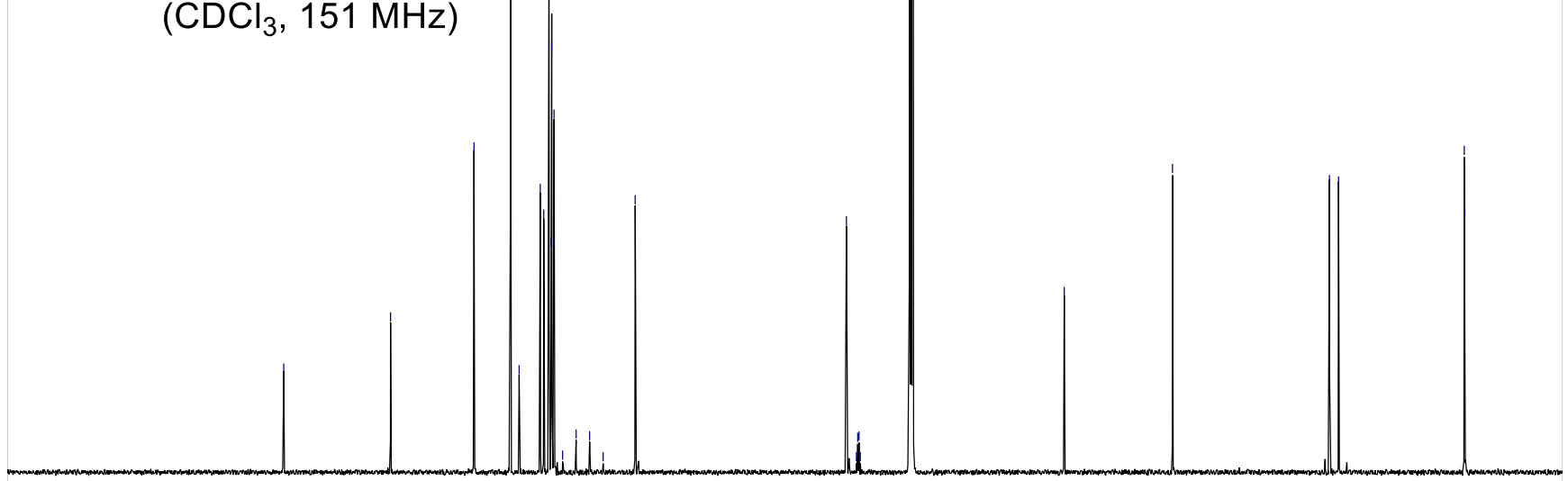



D. Rhoades, * et al.
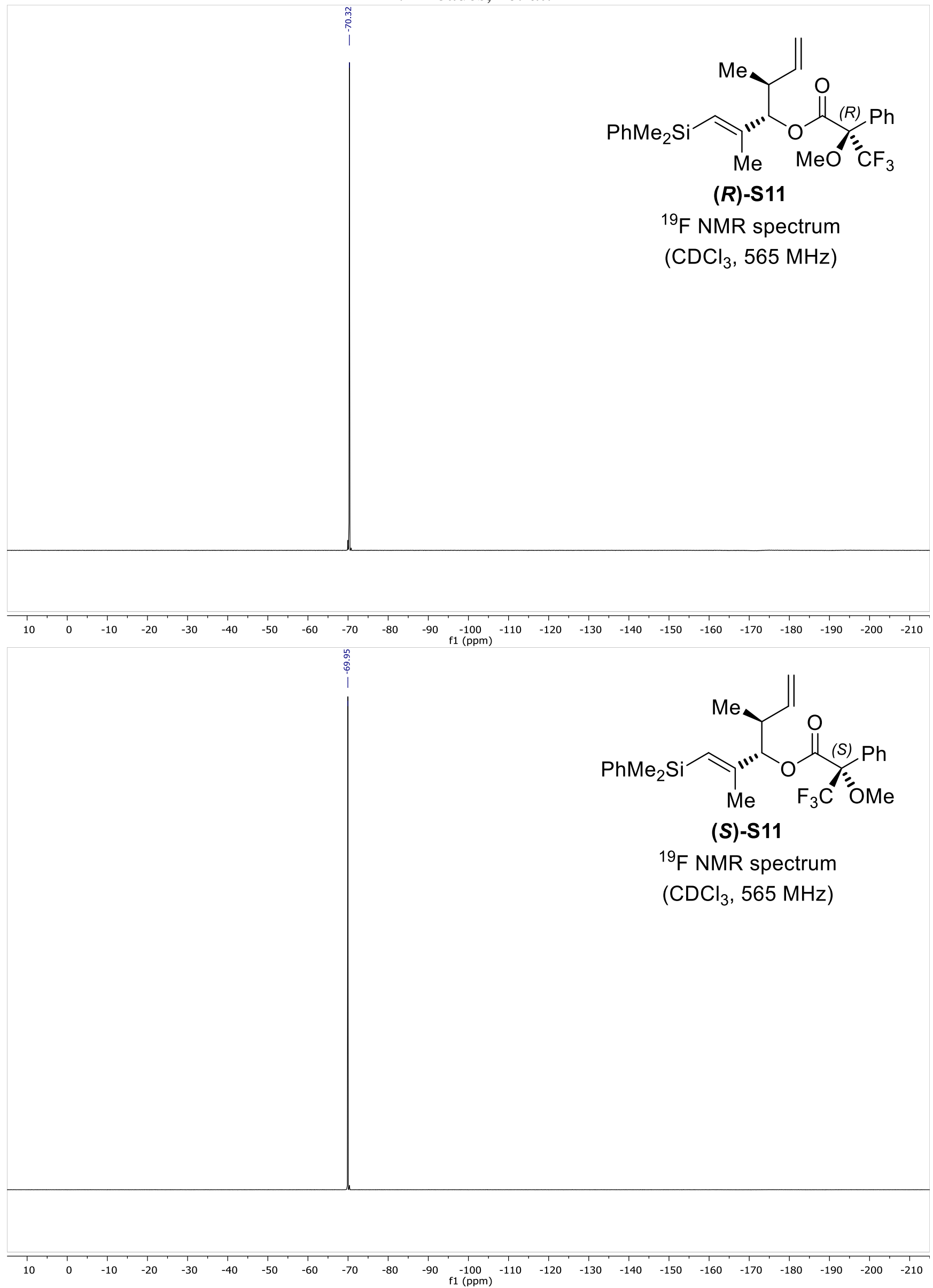


\section{Rhoades,* et al.}<smiles>CCC(C)OC(=O)CC(=O)CC/C(C)=C\I</smiles>

13

${ }^{1} \mathrm{H}$ NMR spectrum

$\left(\mathrm{CDCl}_{3}, 600 \mathrm{MHz}\right)$
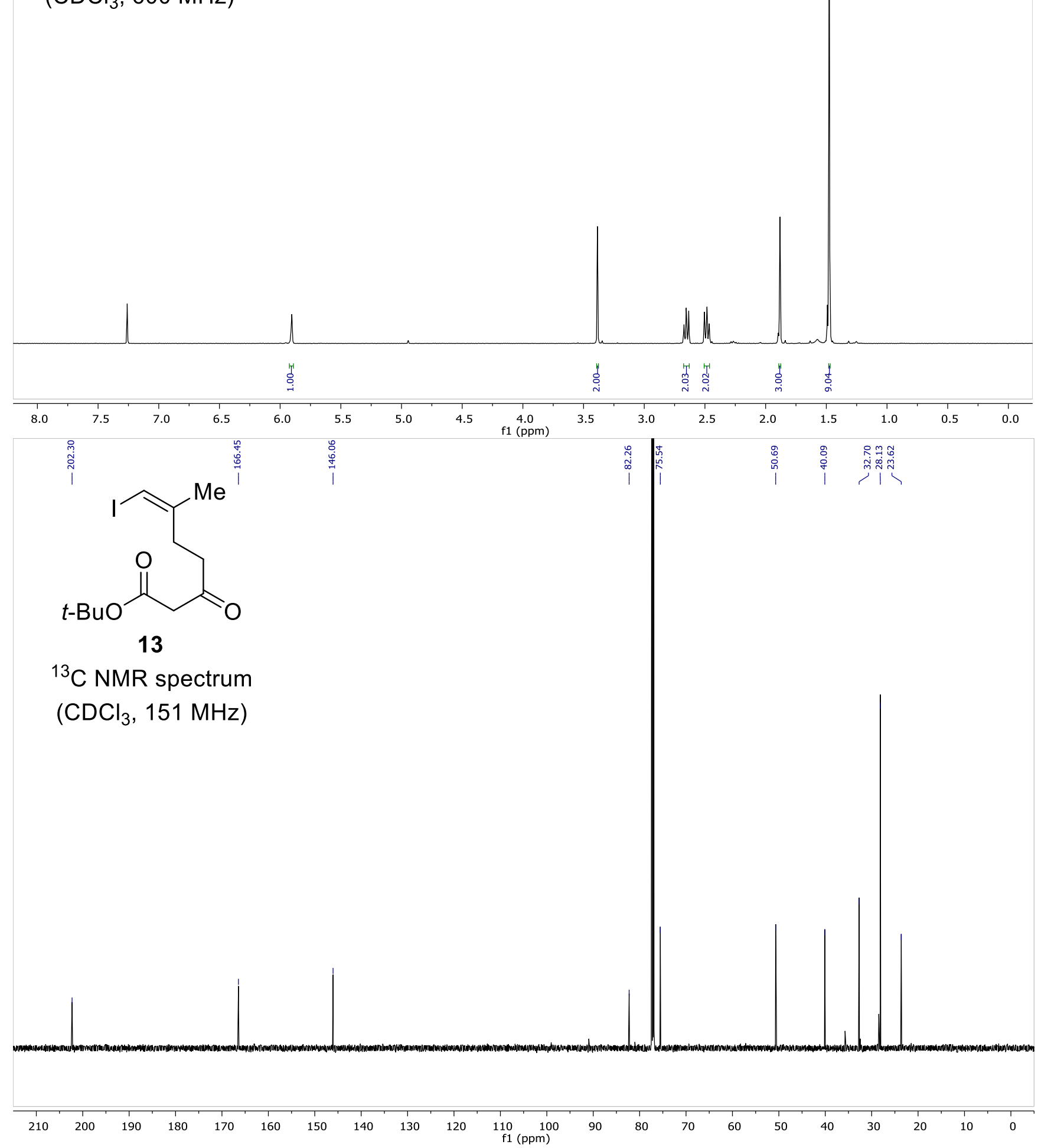
D. Rhoades, * et al.

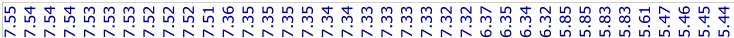<smiles>CCCCOC(=O)CC(=O)CC/C(C)=C\C=C/[C@H](C)[C@H](O)/C(C)=C/[SiH2]C(C)(C)C</smiles>

14

${ }^{1} \mathrm{H}$ NMR spectrum

$\left(\mathrm{CDCl}_{3}, 600 \mathrm{MHz}\right)$

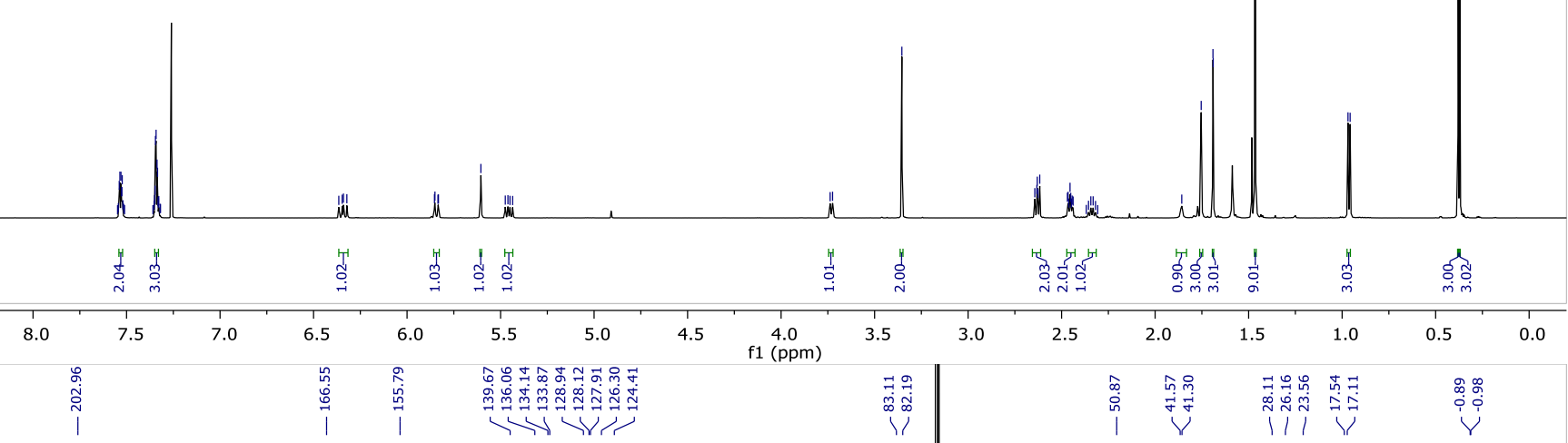<smiles>CCCCCOC(=O)CC(=O)CC/C(C)=C\C=C/[C@H](C)[C@@H](O)/C(C)=C/[SiH2]P</smiles>

14

${ }^{13} \mathrm{C}$ NMR spectrum $\left(\mathrm{CDCl}_{3}, 151 \mathrm{MHz}\right)$

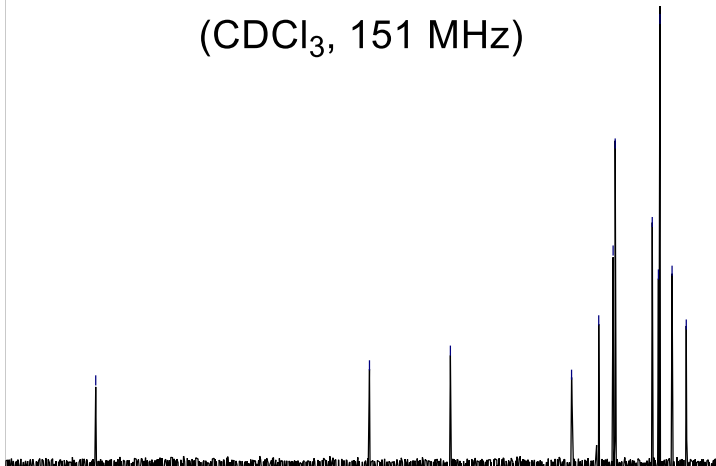




\section{Rhoades, * et al.}
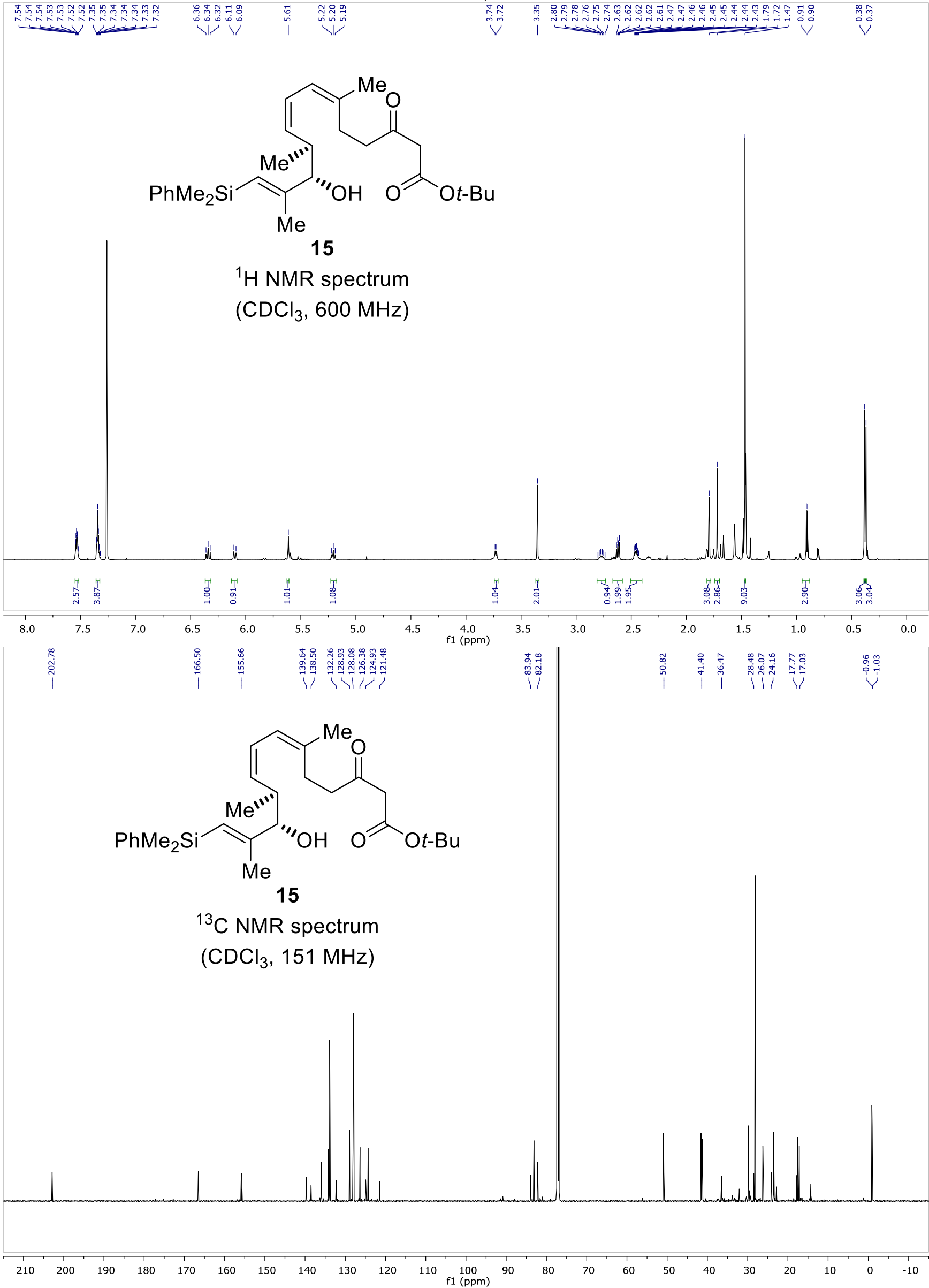


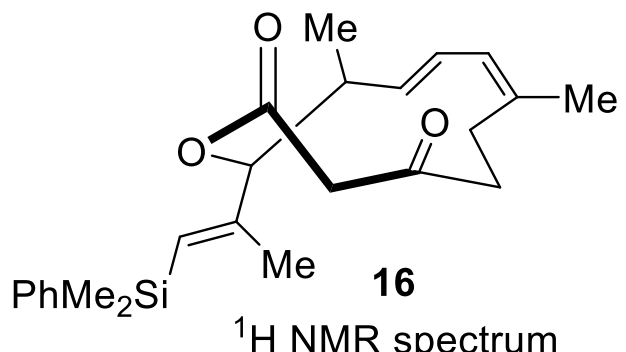

${ }^{1} \mathrm{H}$ NMR spectrum

$\left(\mathrm{CDCl}_{3}, 600 \mathrm{MHz}\right)$
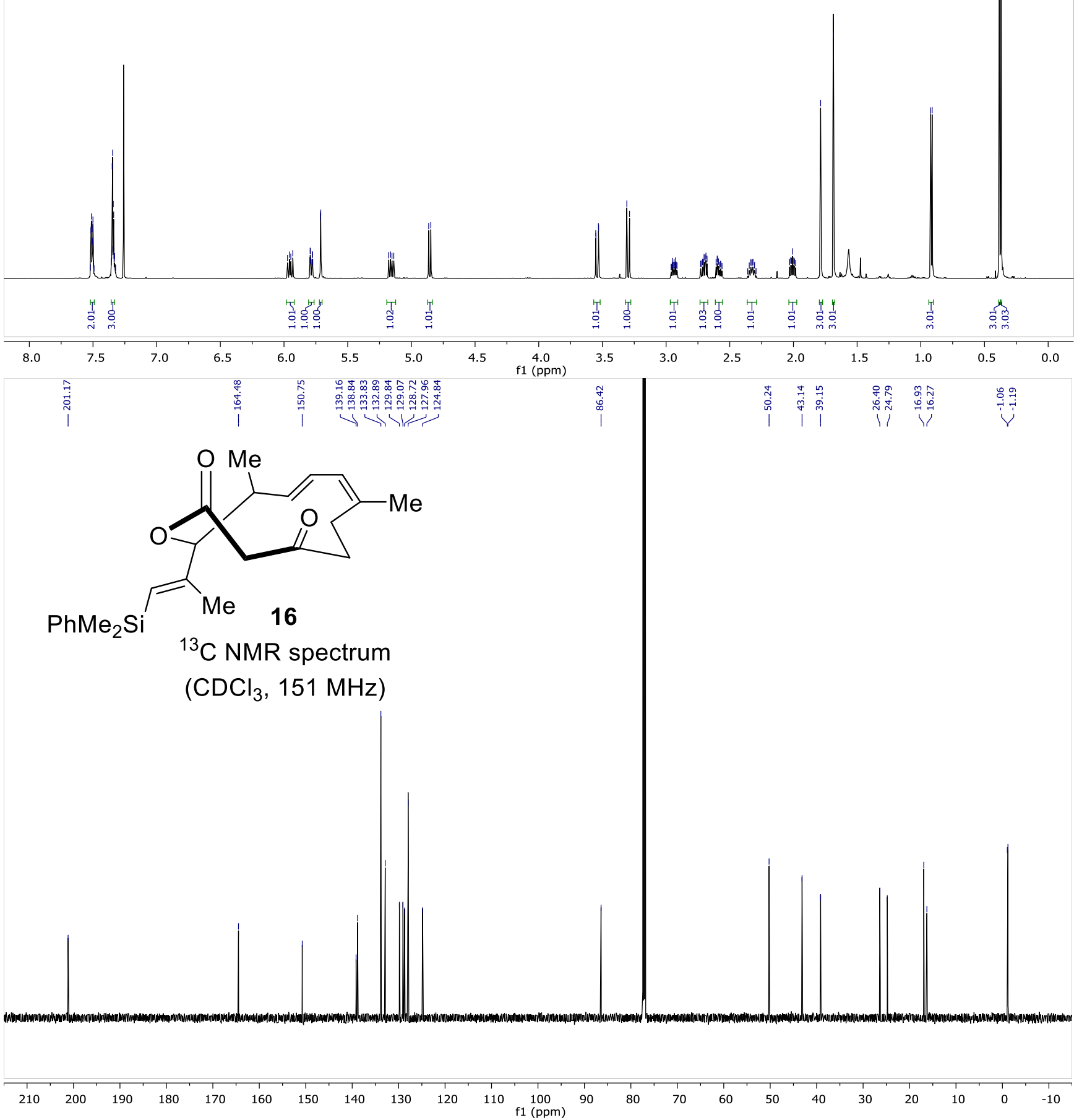
D. Rhoades,* et al.

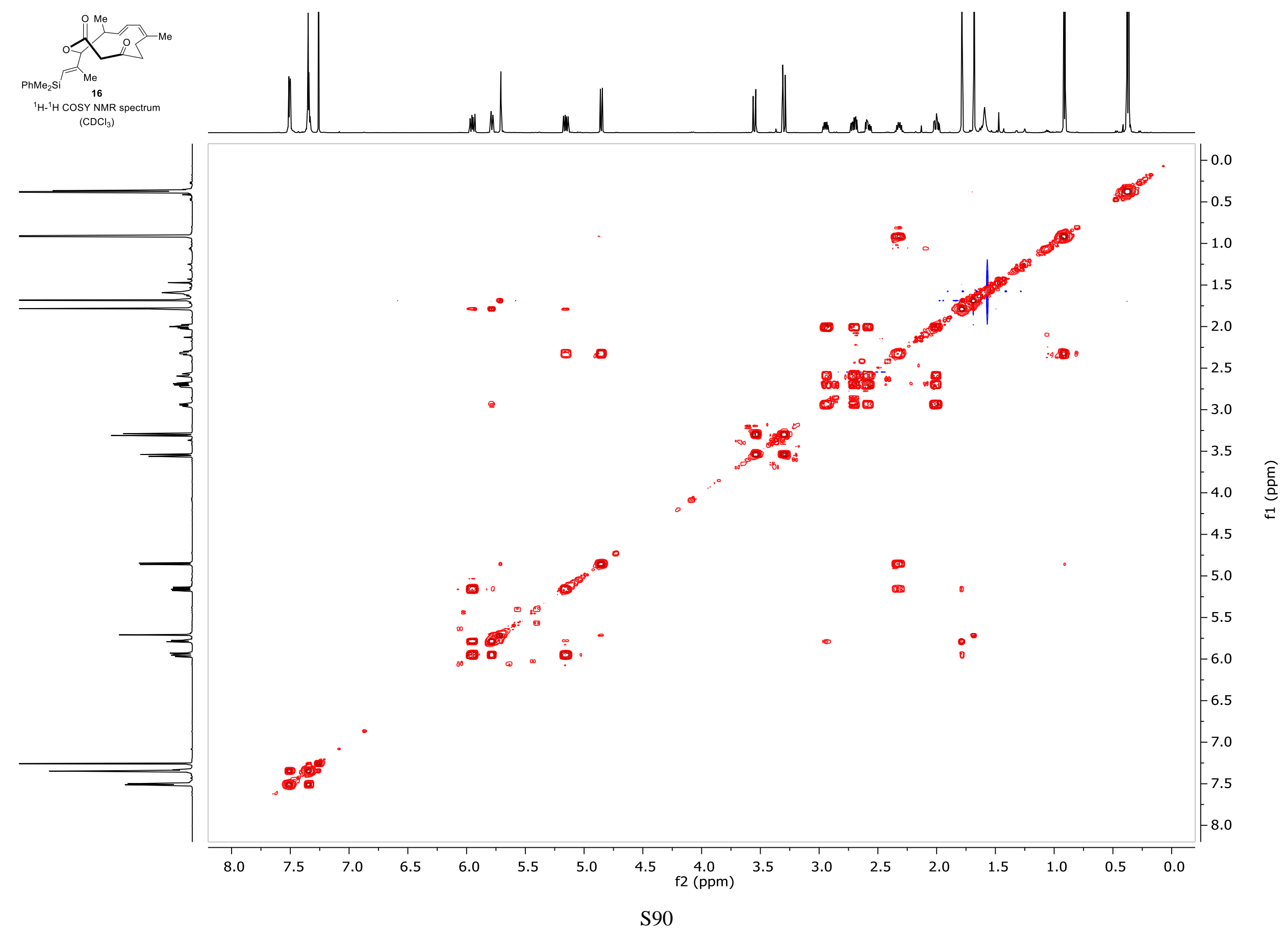


D. Rhoades, * et al.

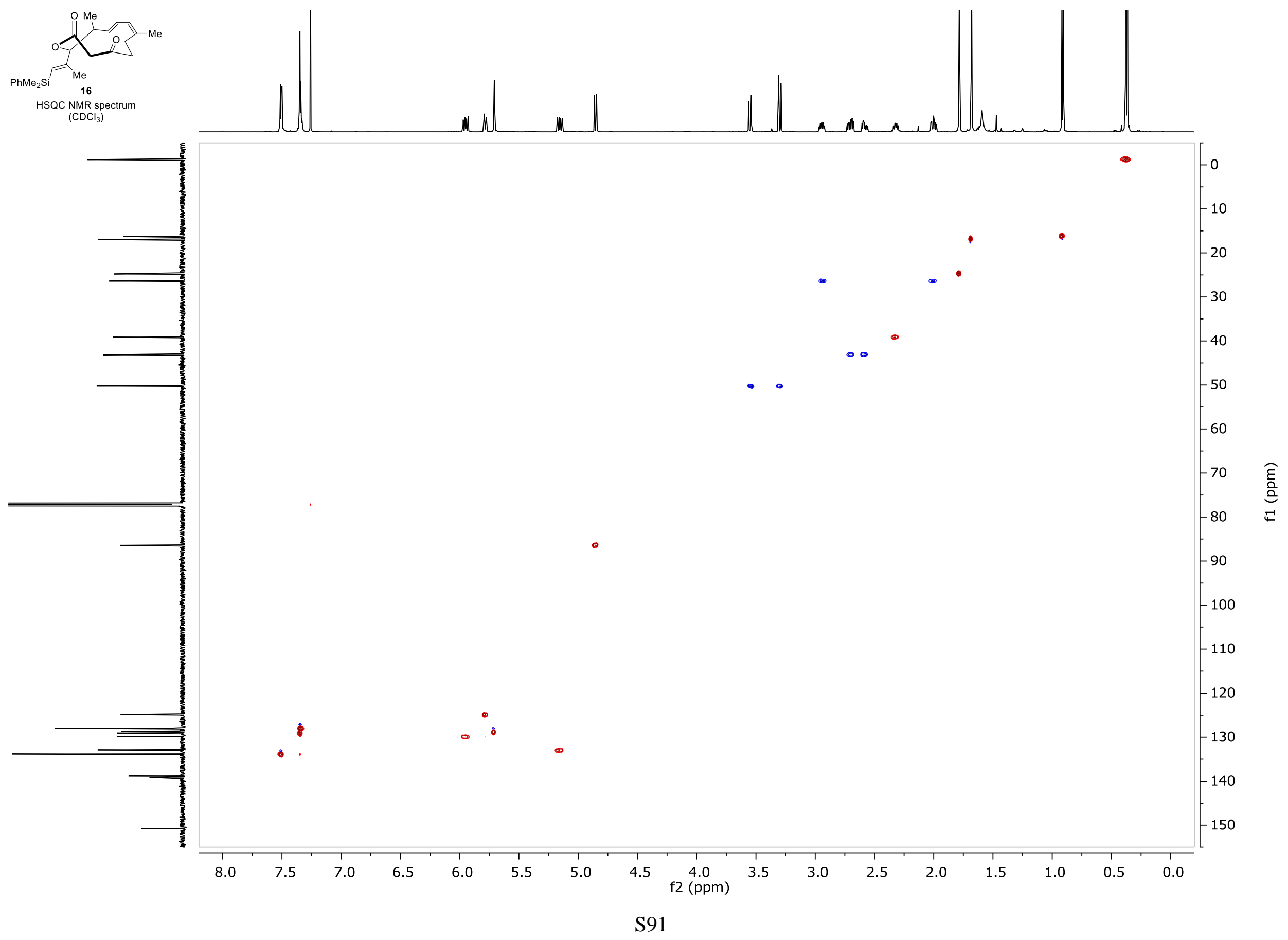


D. Rhoades,* et al.

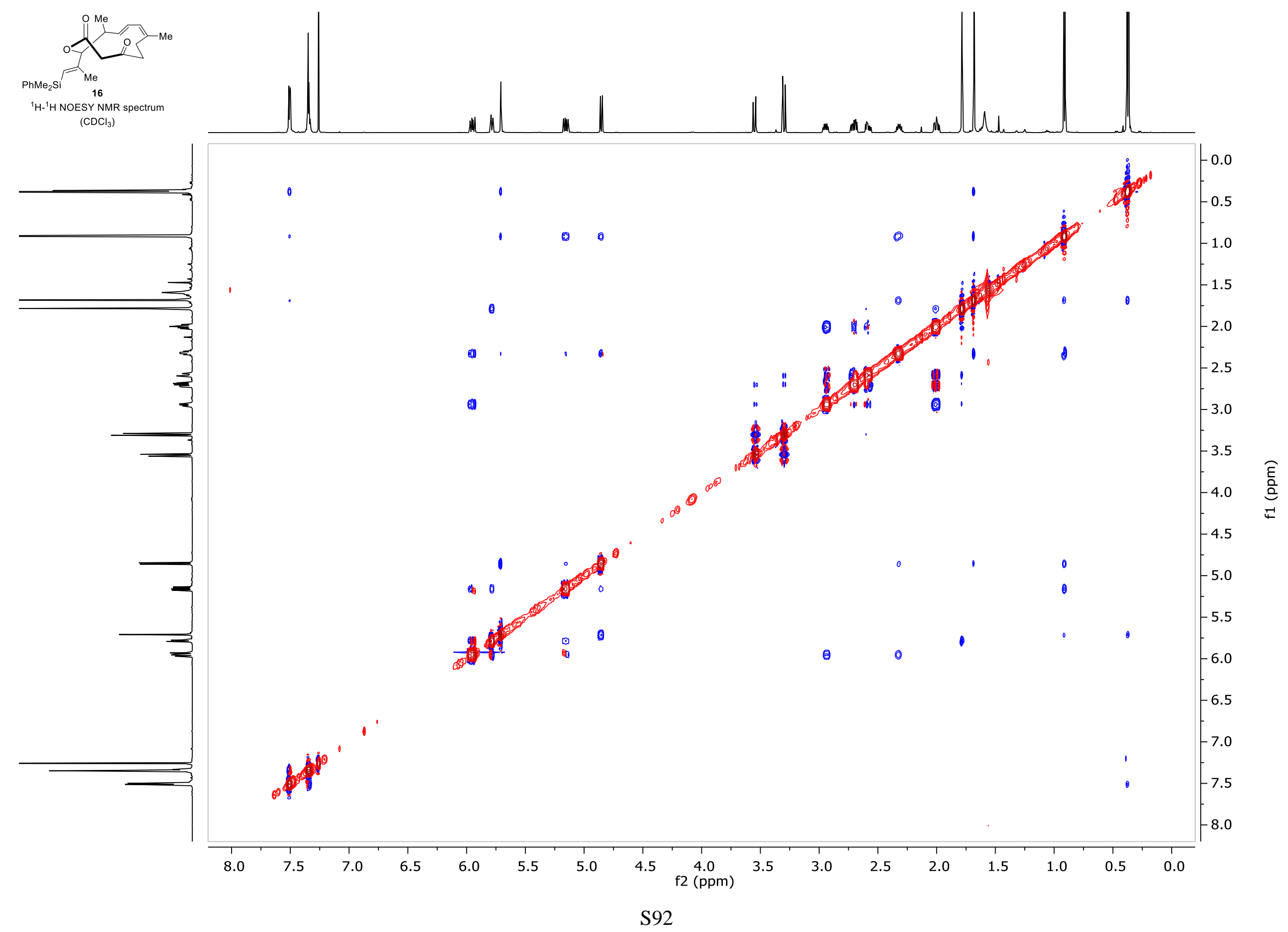



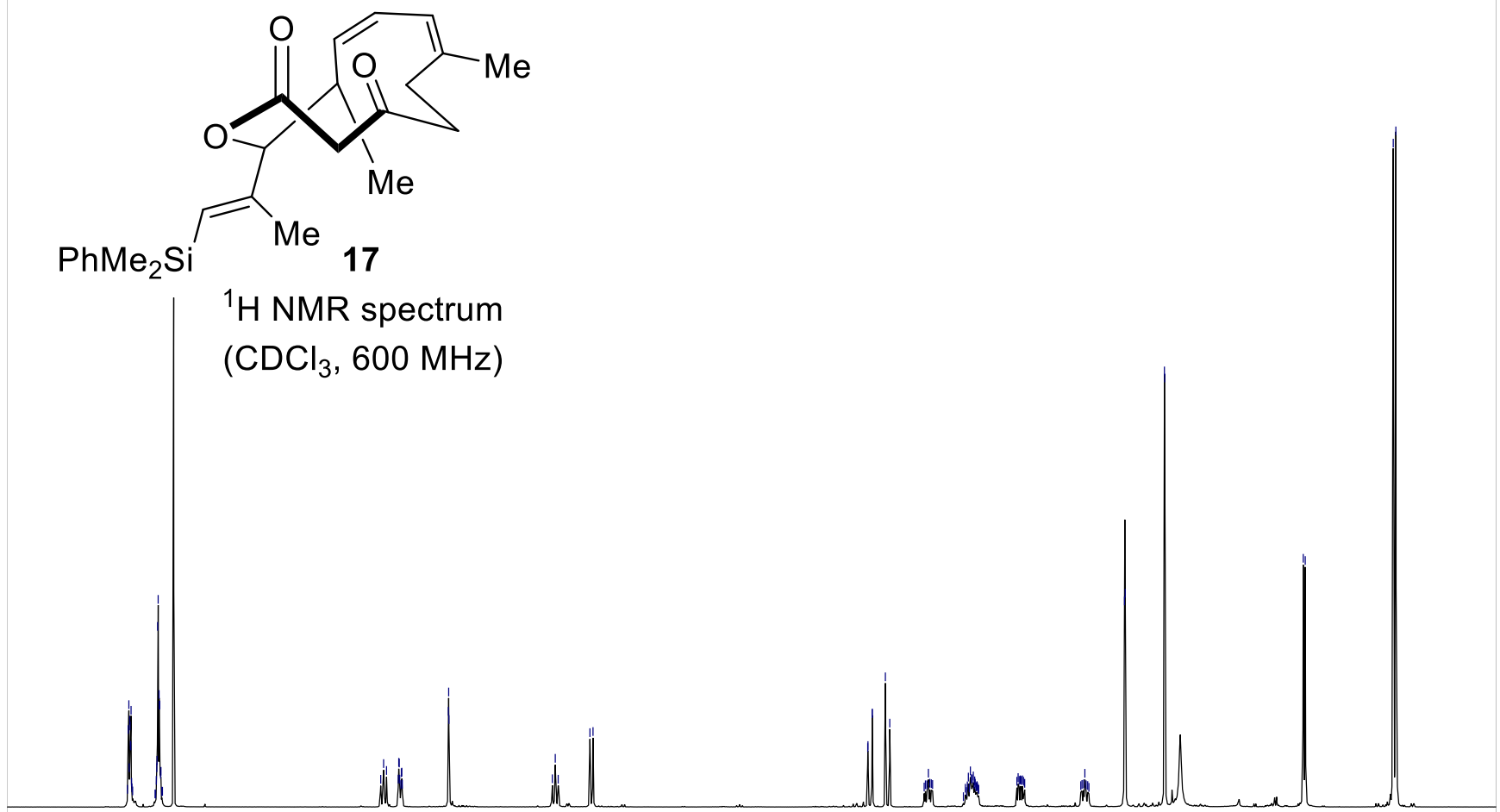

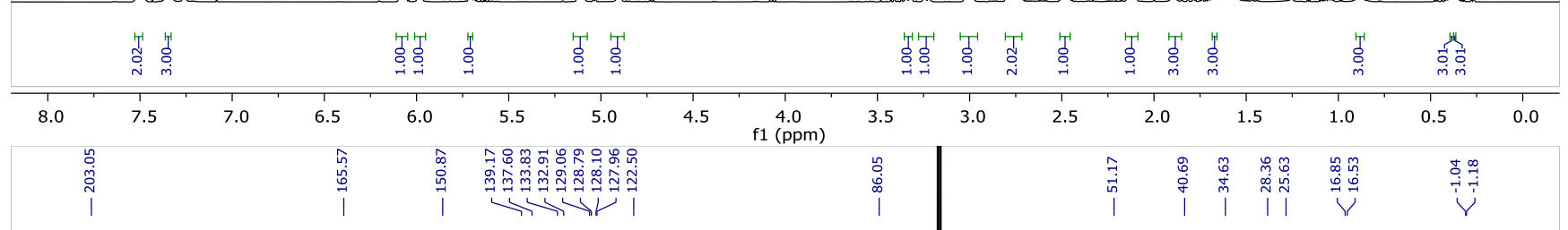

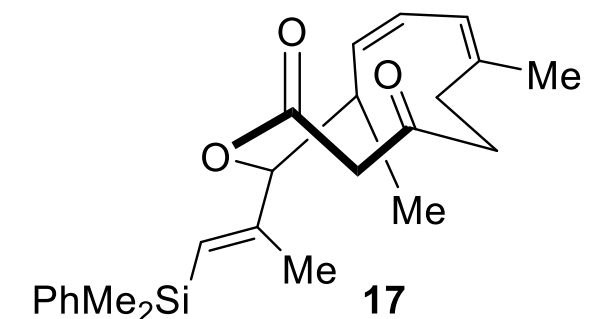

${ }^{13} \mathrm{C}$ NMR spectrum

$\left(\mathrm{CDCl}_{3}, 151 \mathrm{MHz}\right)$
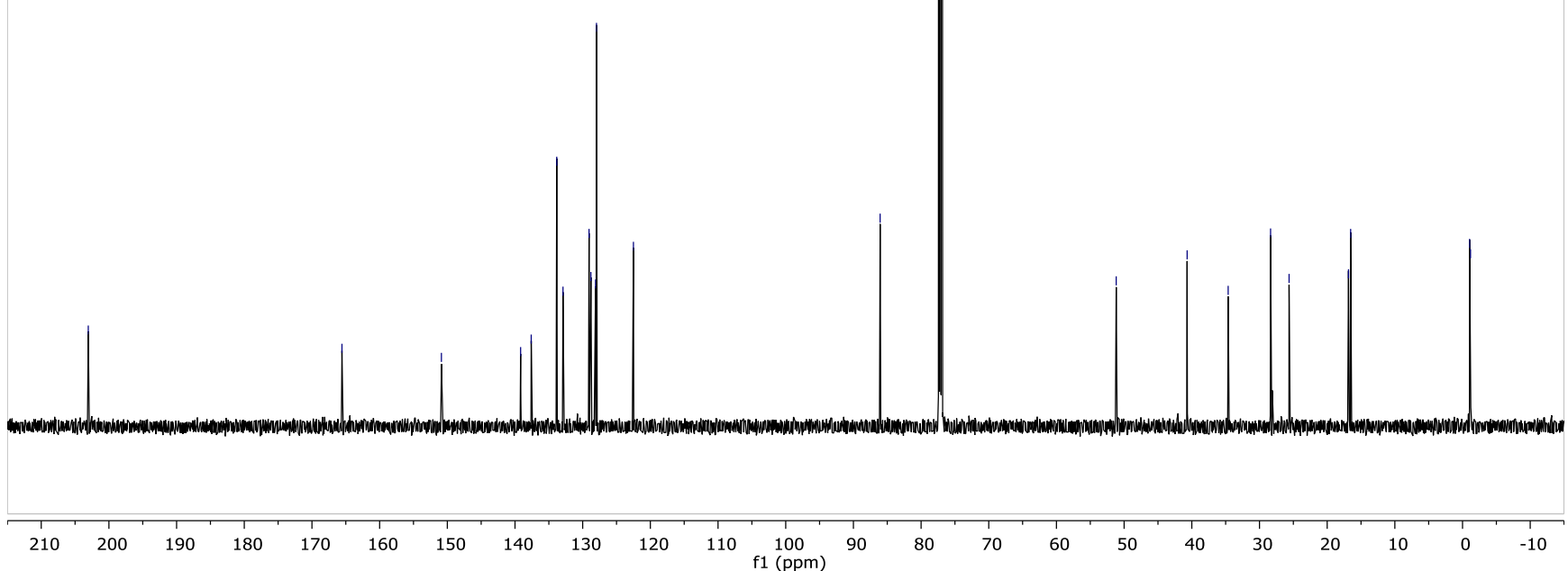
D. Rhoades, ${ }^{*}$ et al.

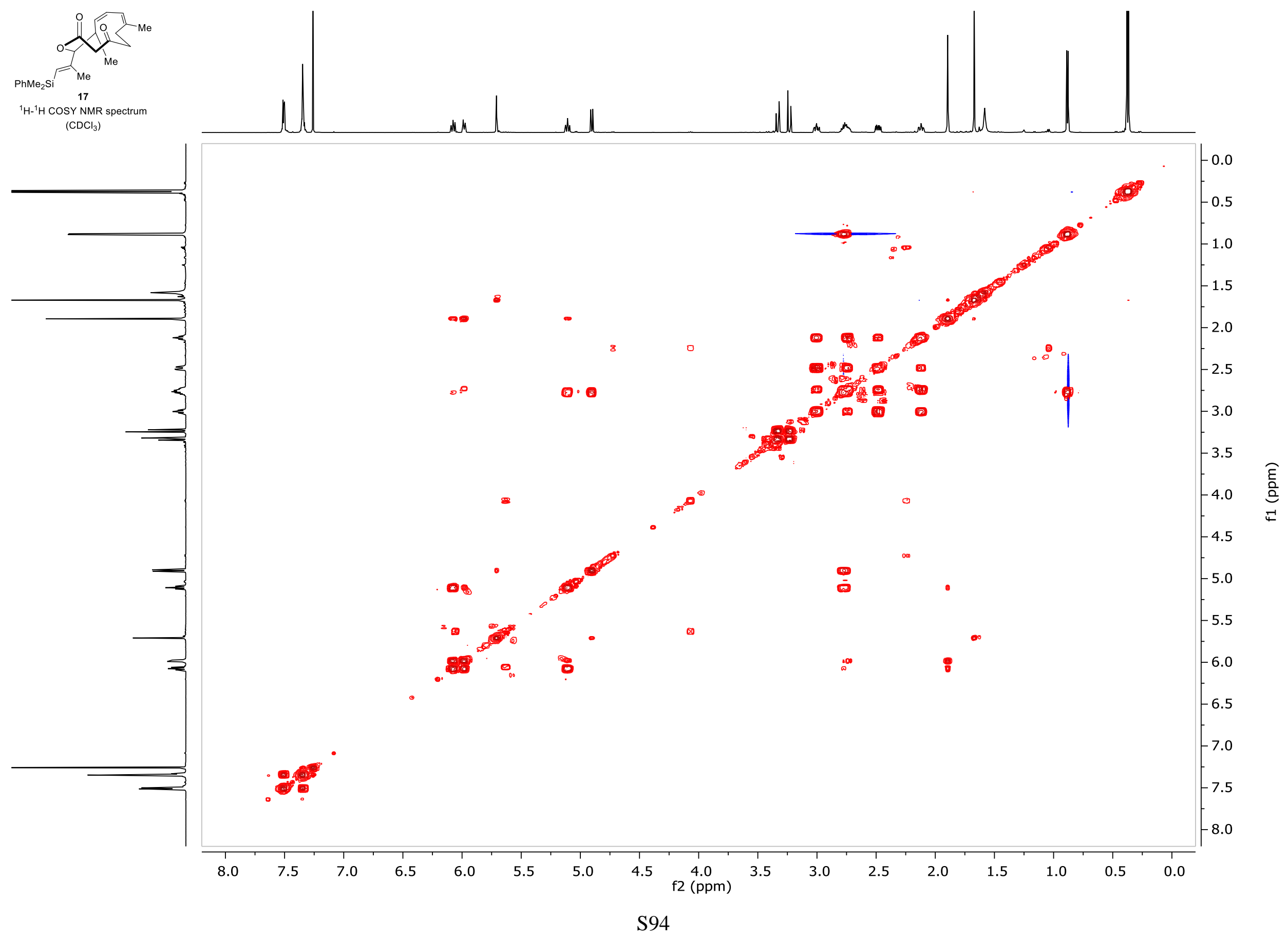


D. Rhoades, * et al.

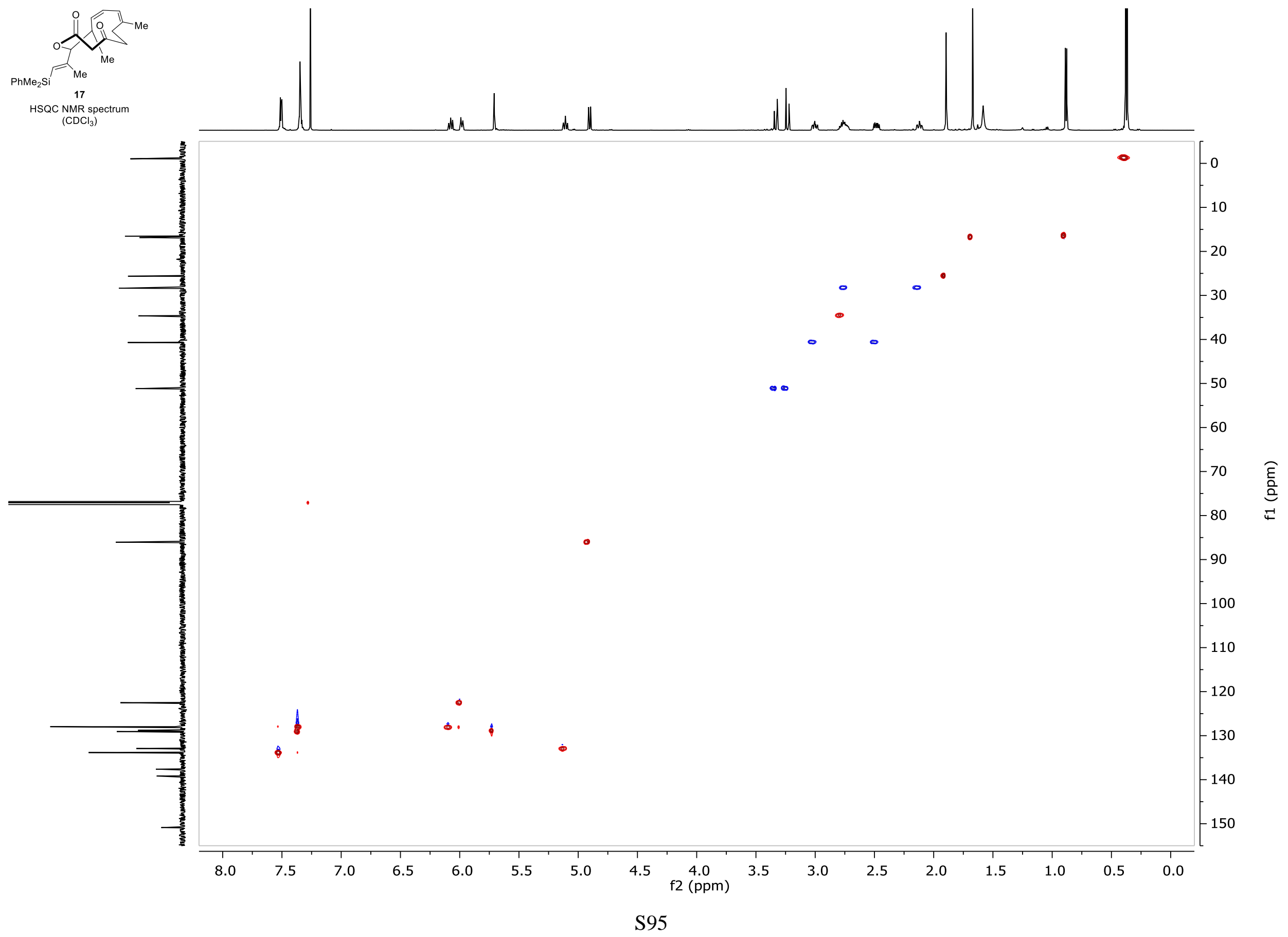


D. Rhoades,* et al.

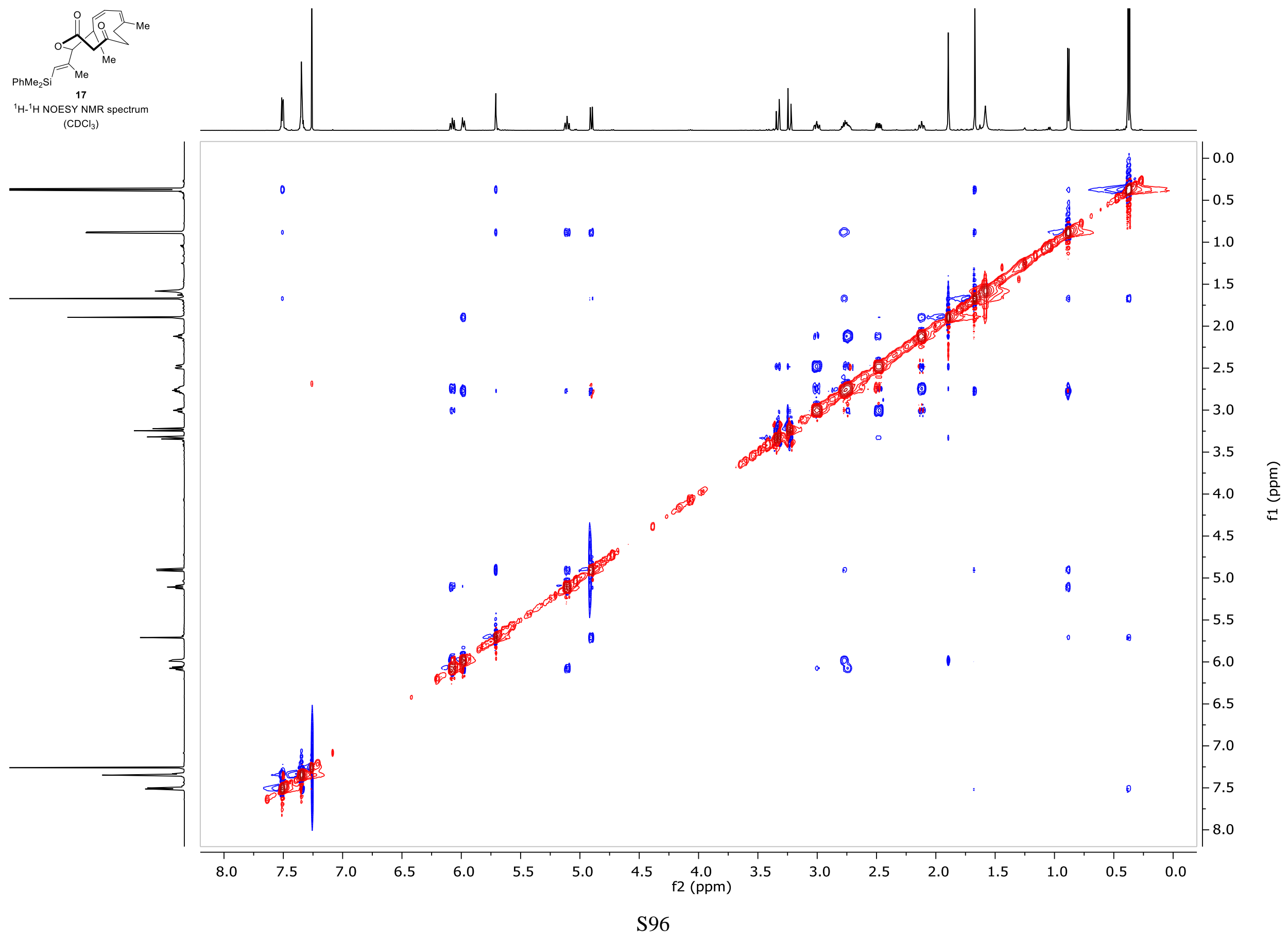




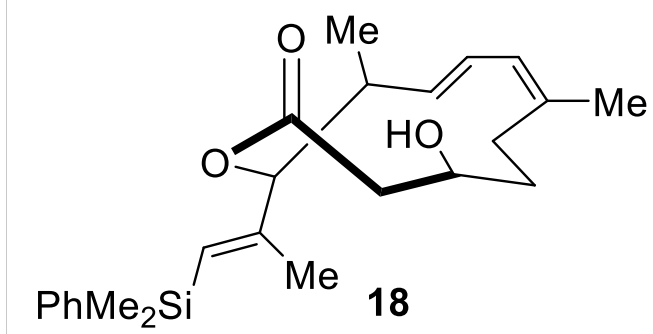

${ }^{1} \mathrm{H}$ NMR spectrum
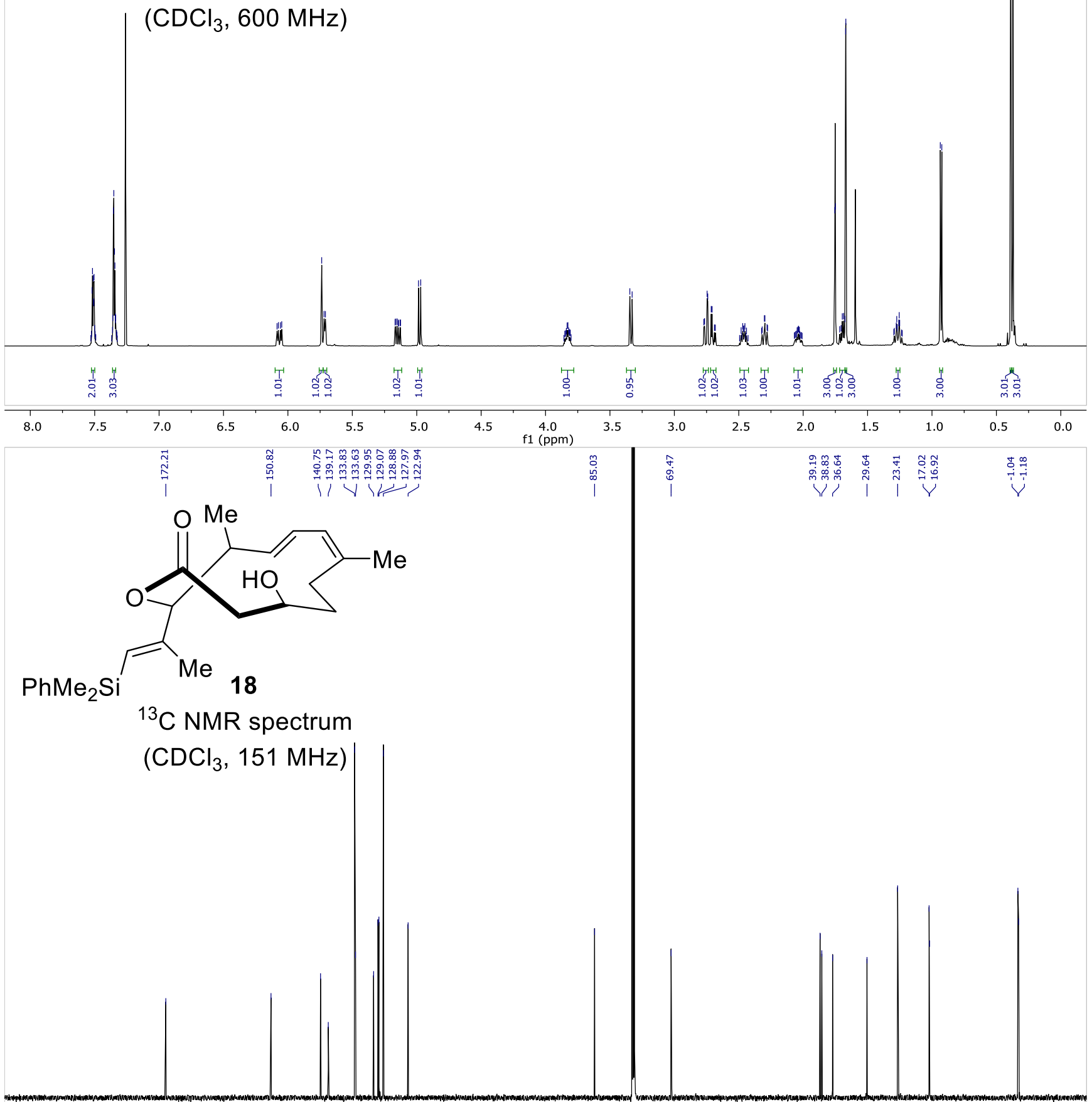
D. Rhoades,* et al.

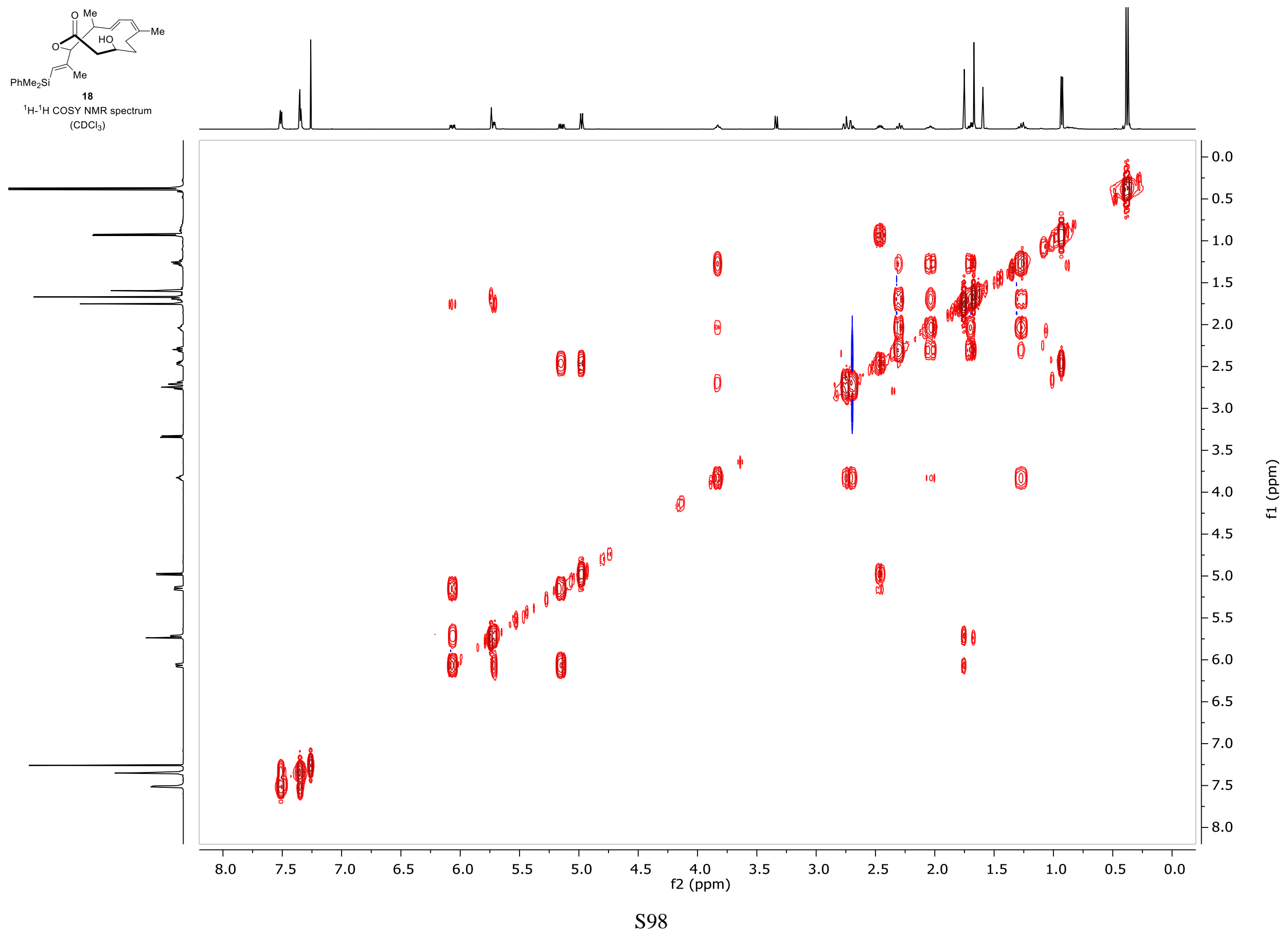


D. Rhoades,* et al.

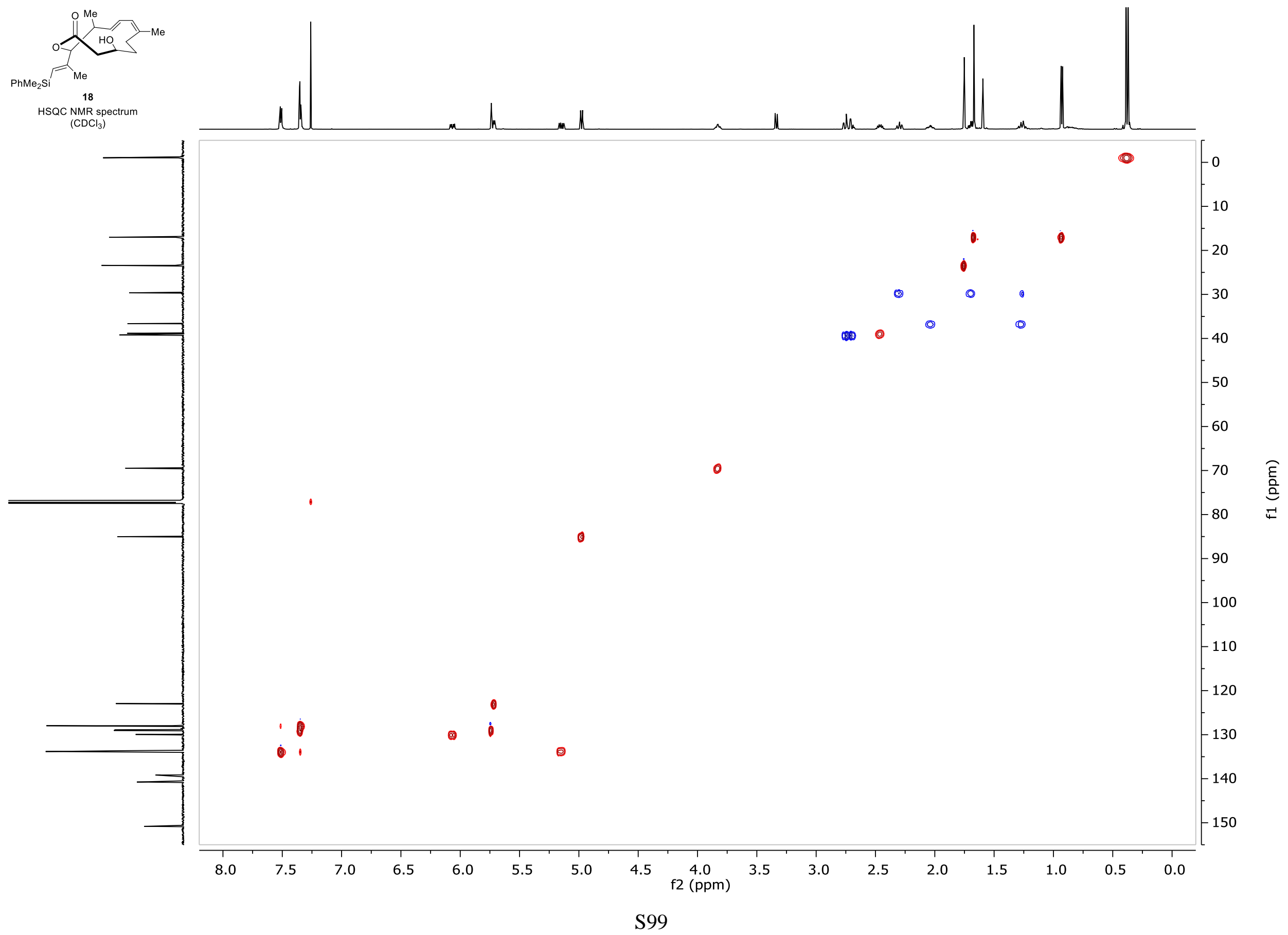



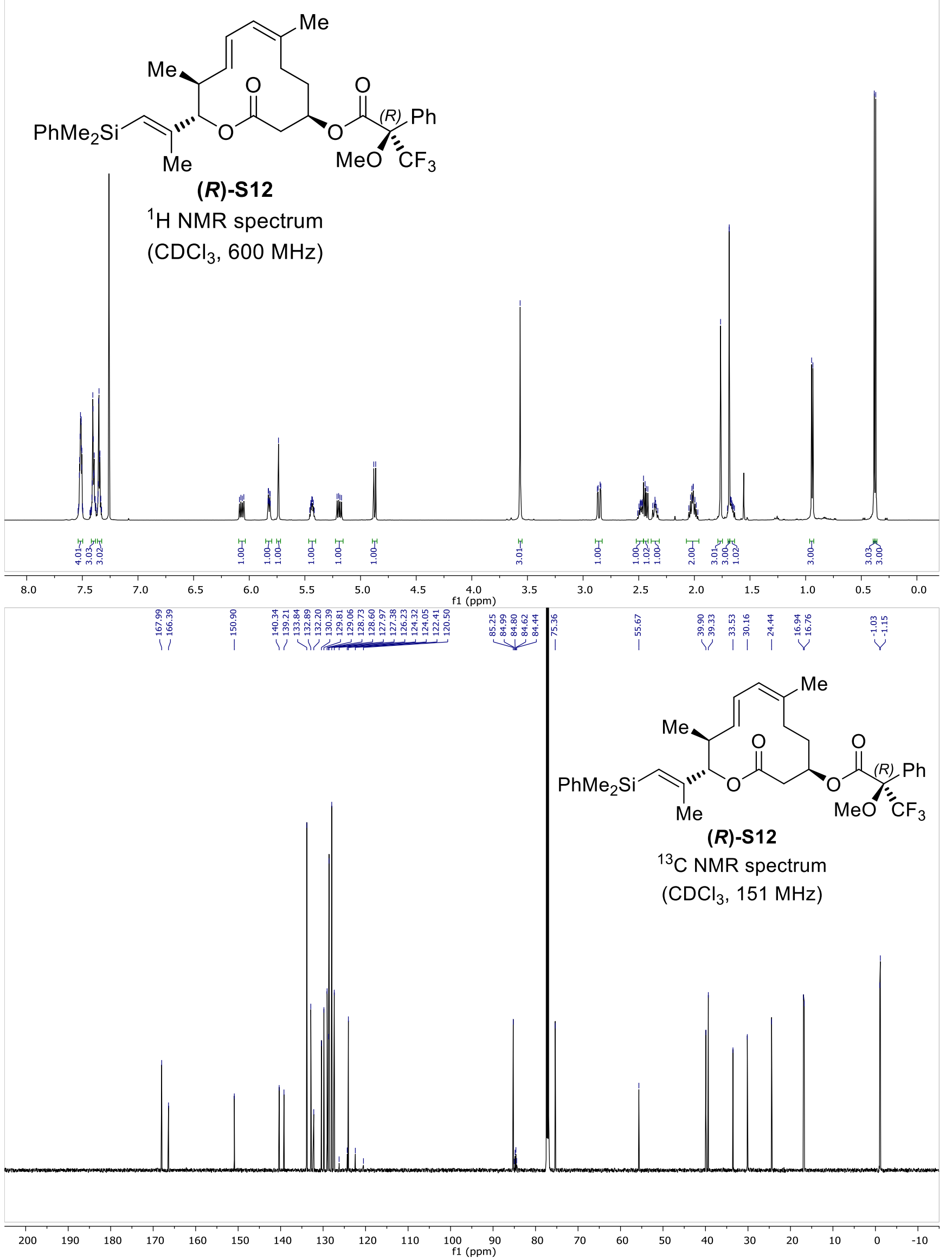
D. Rhoades, ${ }^{*}$ et al.

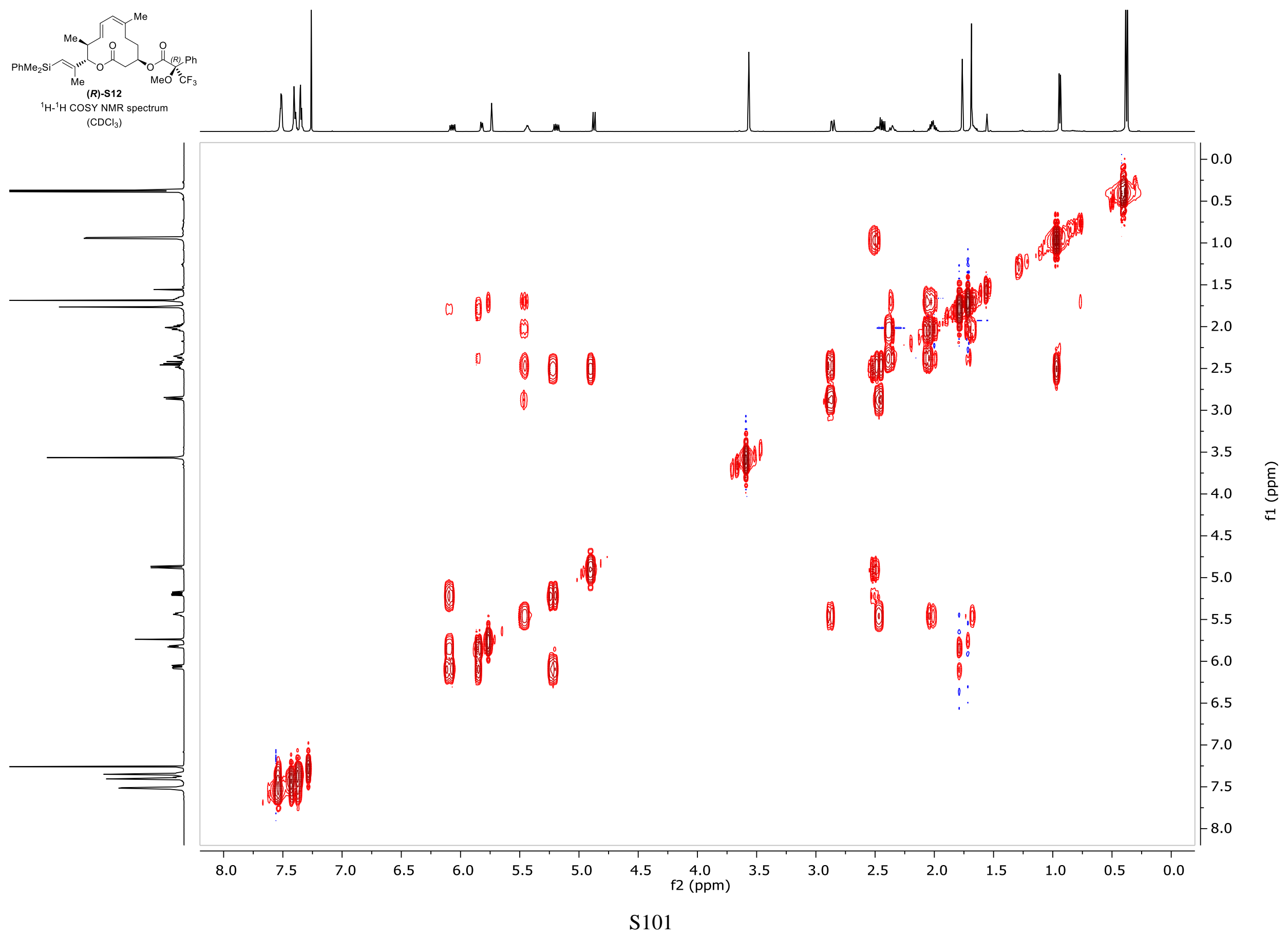


D. Rhoades, * et al.

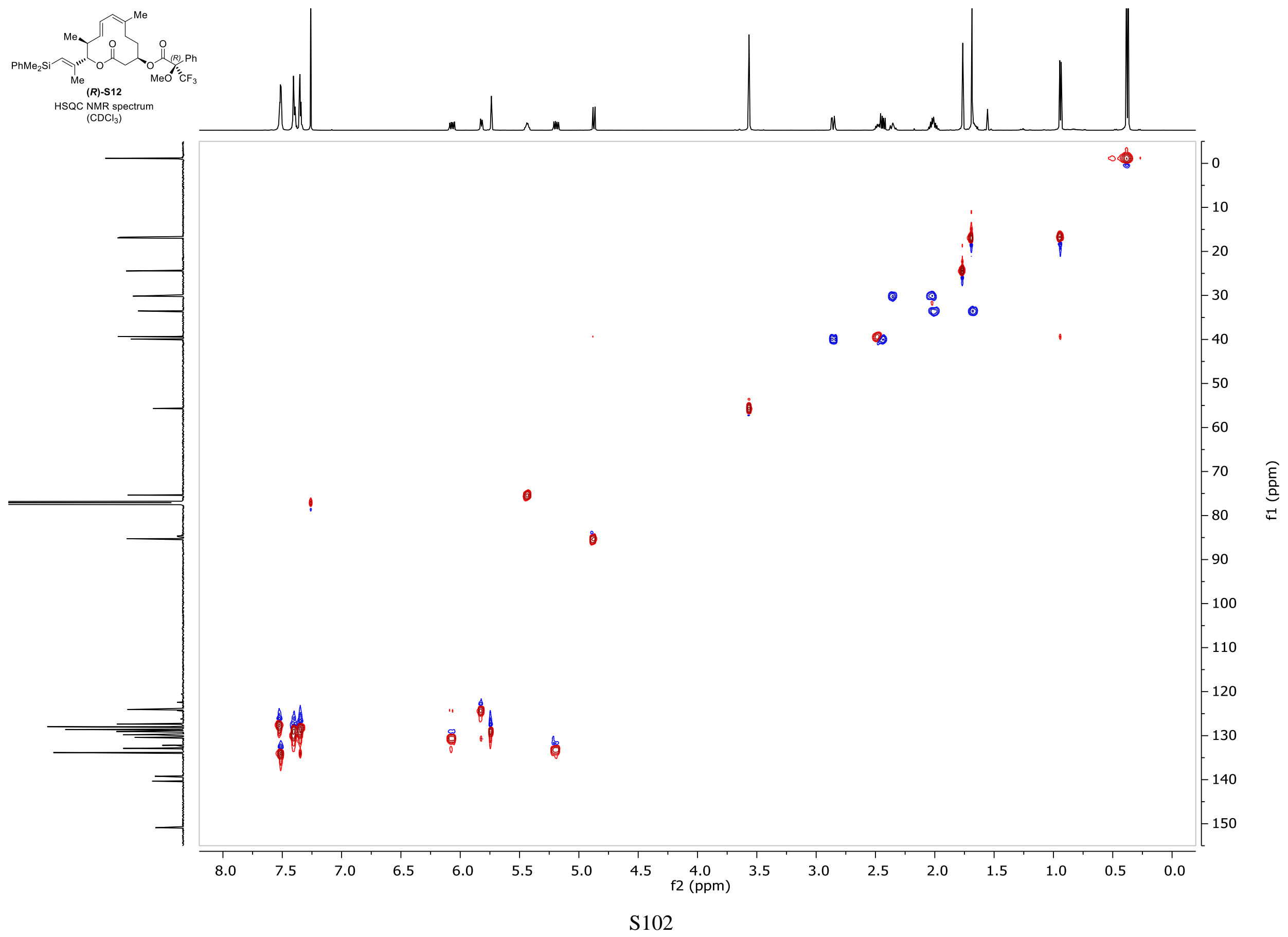


D. Rhoades, ${ }^{*}$ et al.

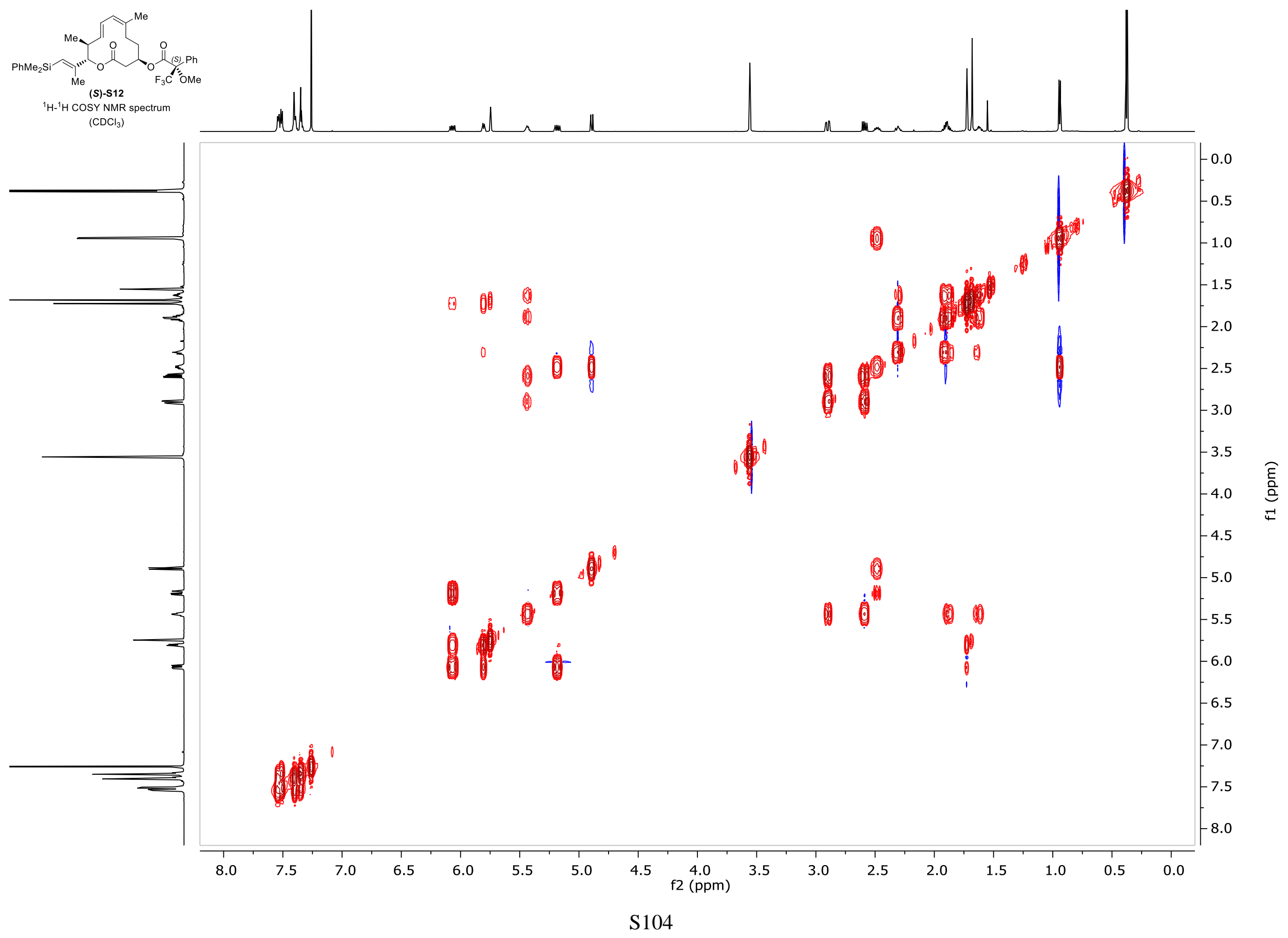


D. Rhoades, * et al.

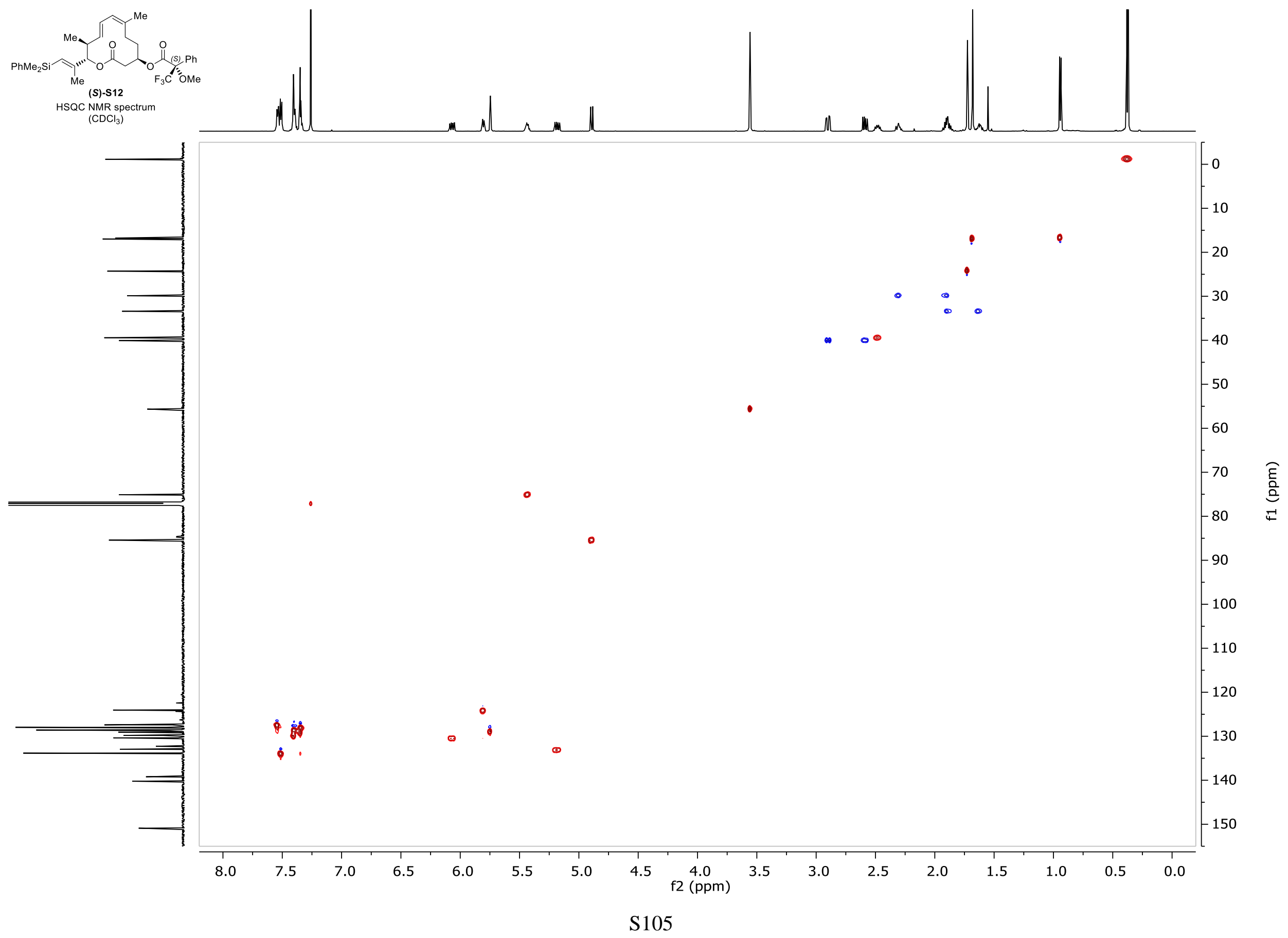



D. Rhoades, * et al.
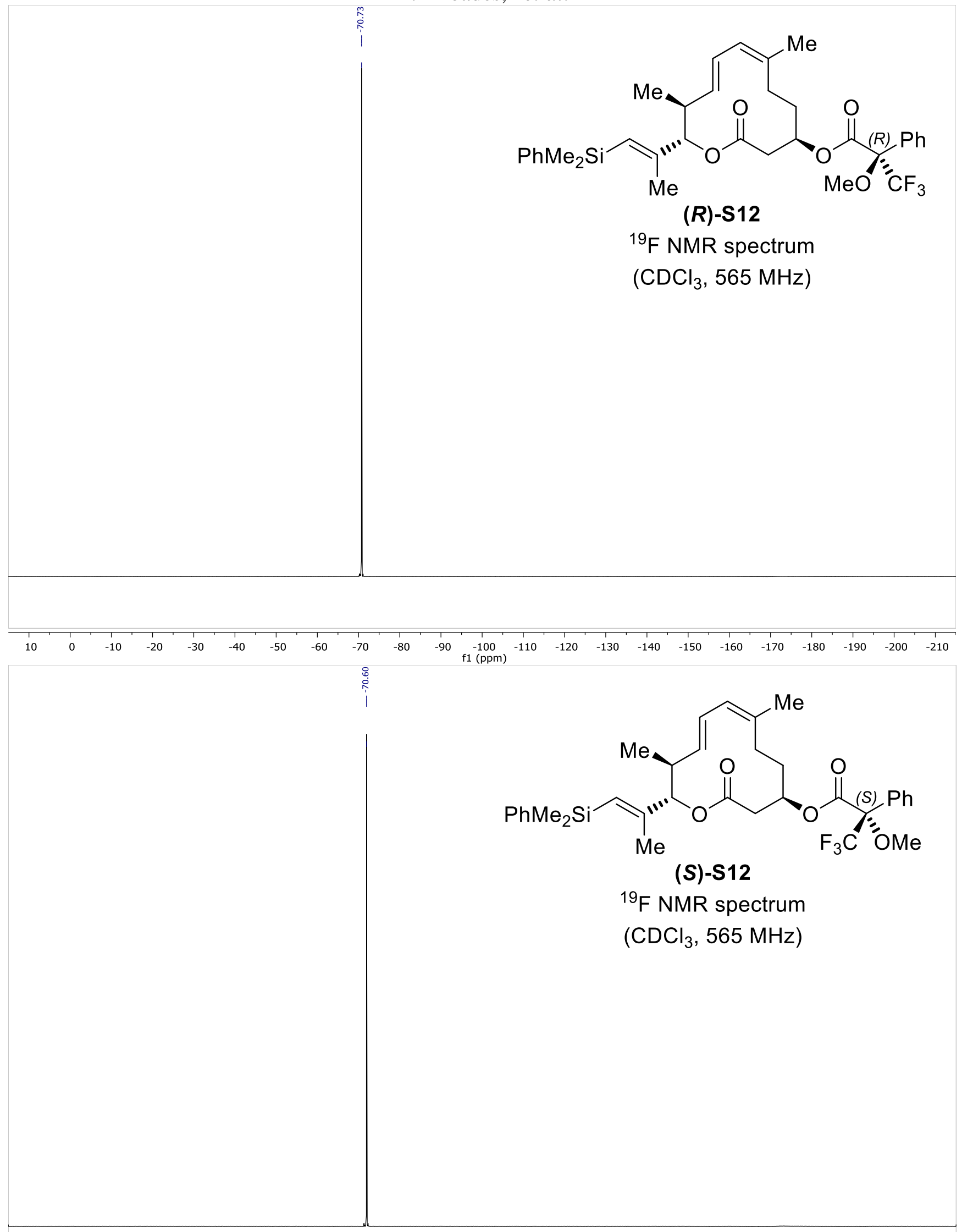


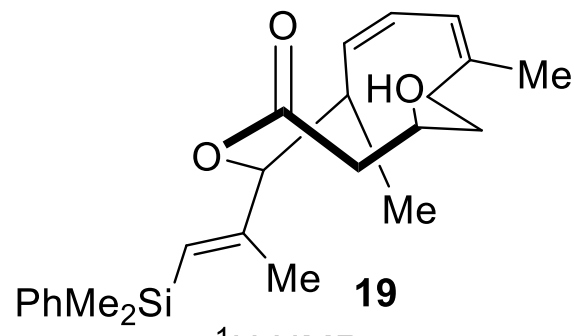

${ }^{1} \mathrm{H}$ NMR spectrum

$\left(\mathrm{CDCl}_{3}, 600 \mathrm{MHz}\right)$
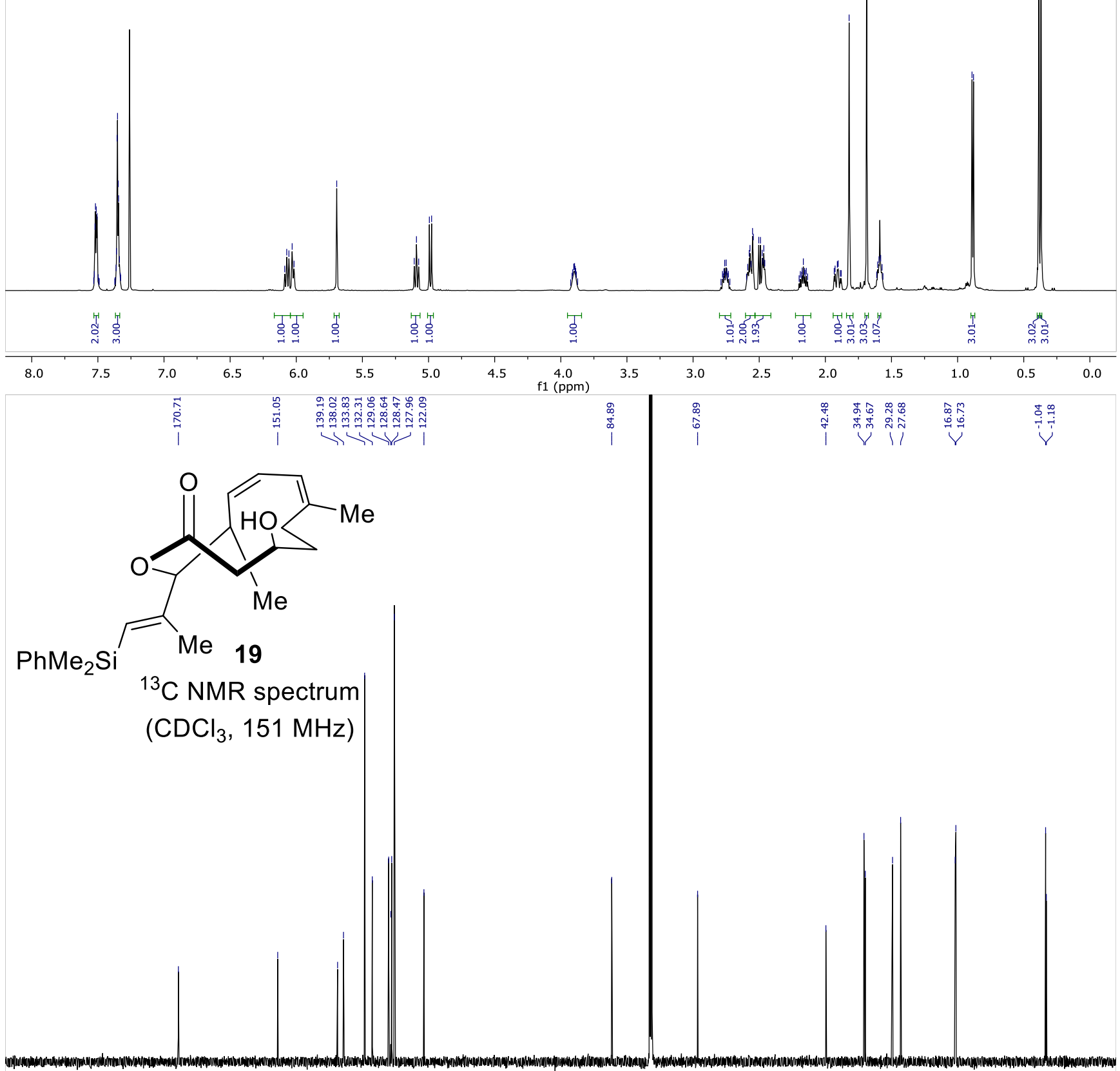
D. Rhoades, ${ }^{*}$ et al.

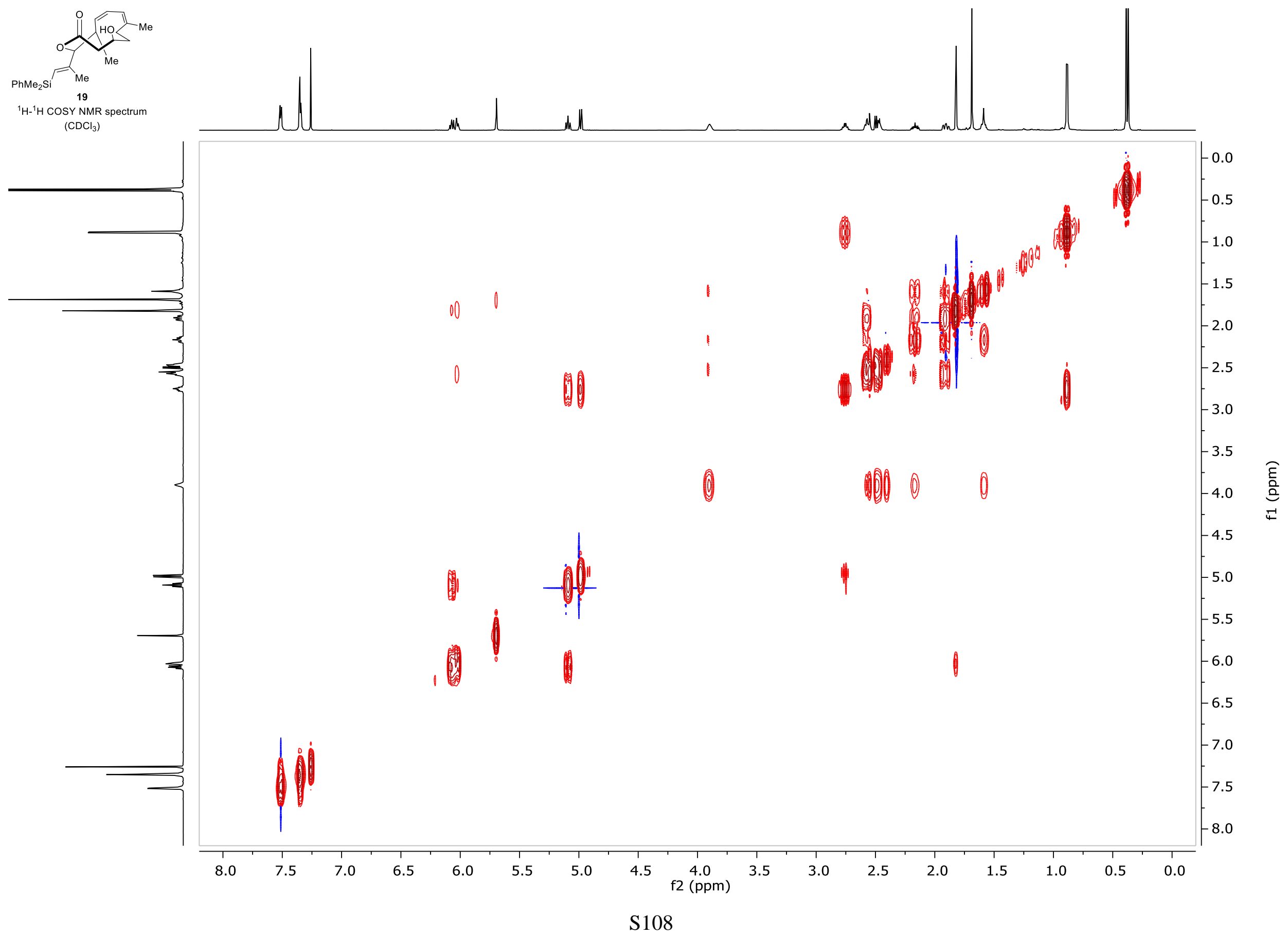


D. Rhoades, * et al.

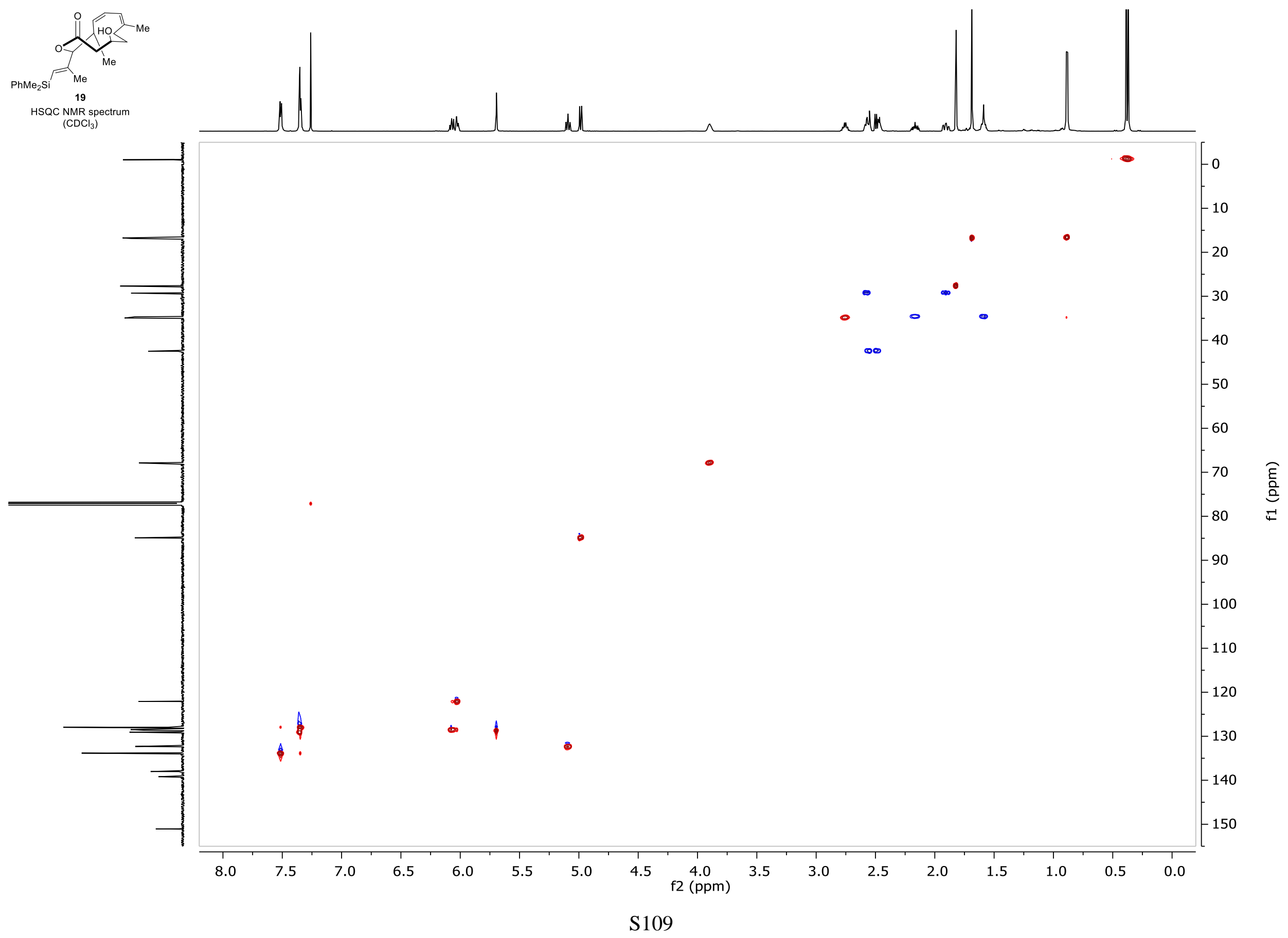


D. Rhoades, * et al.

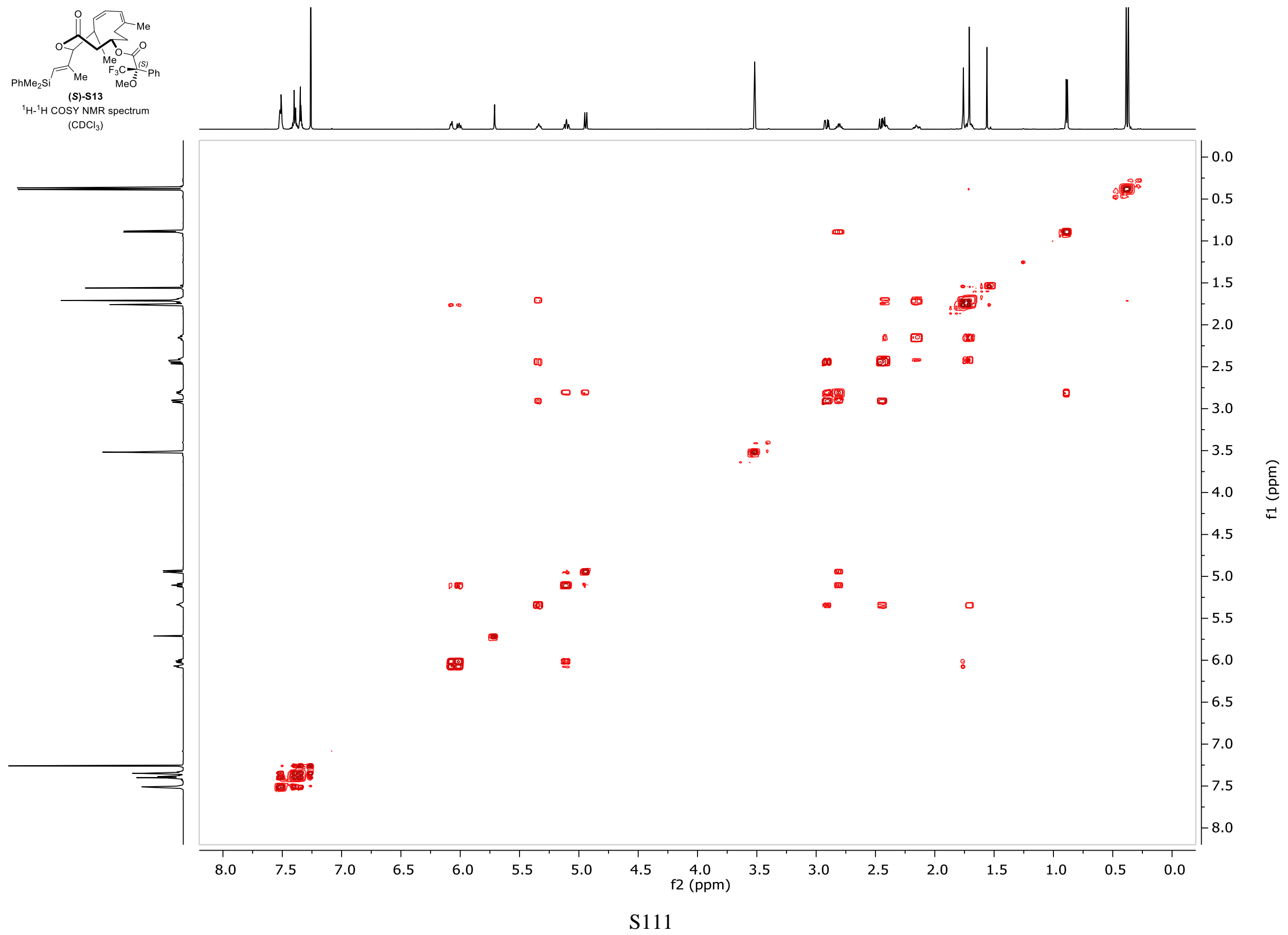



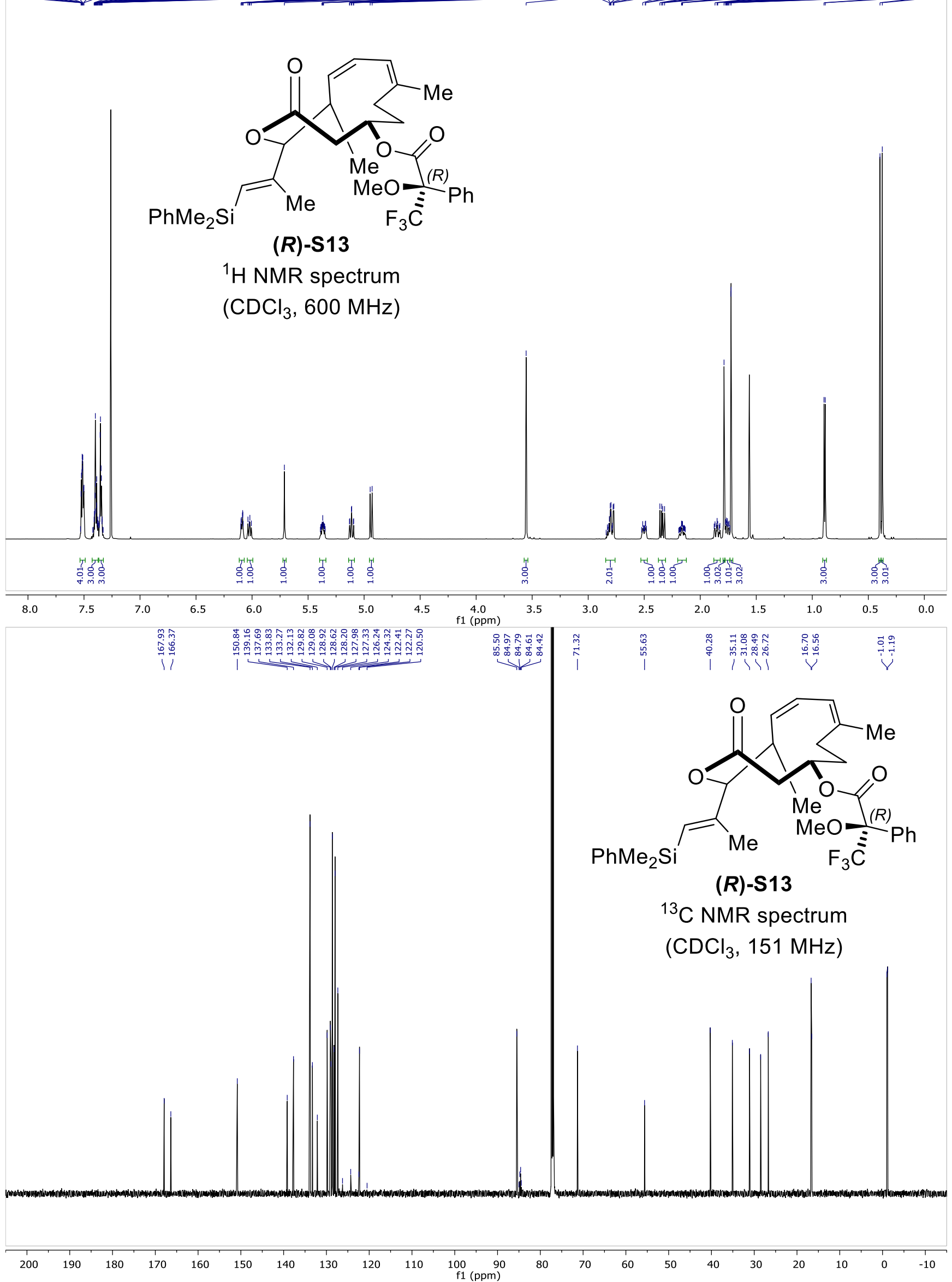
D. Rhoades, * et al.

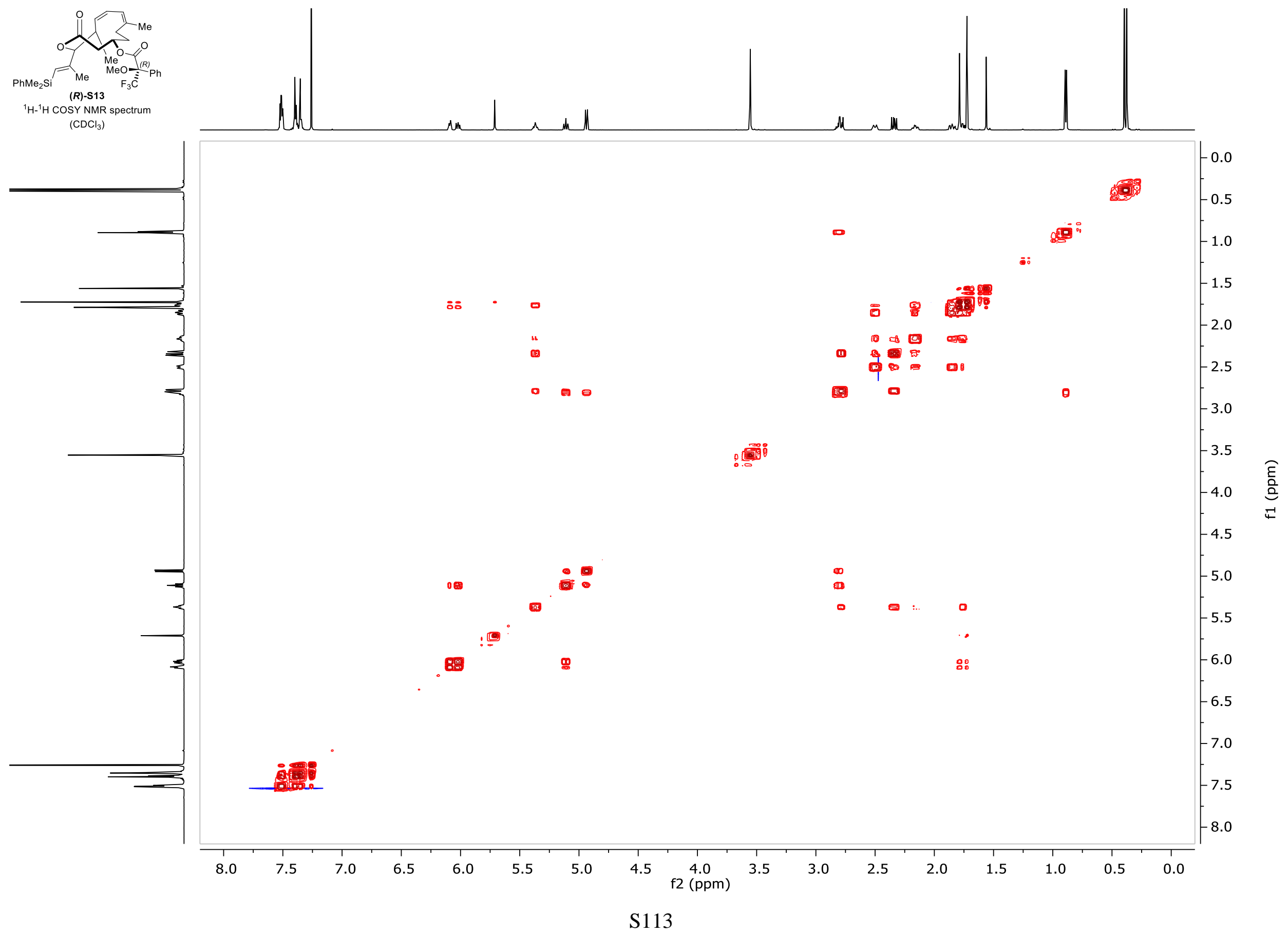



D. Rhoades, * et al.
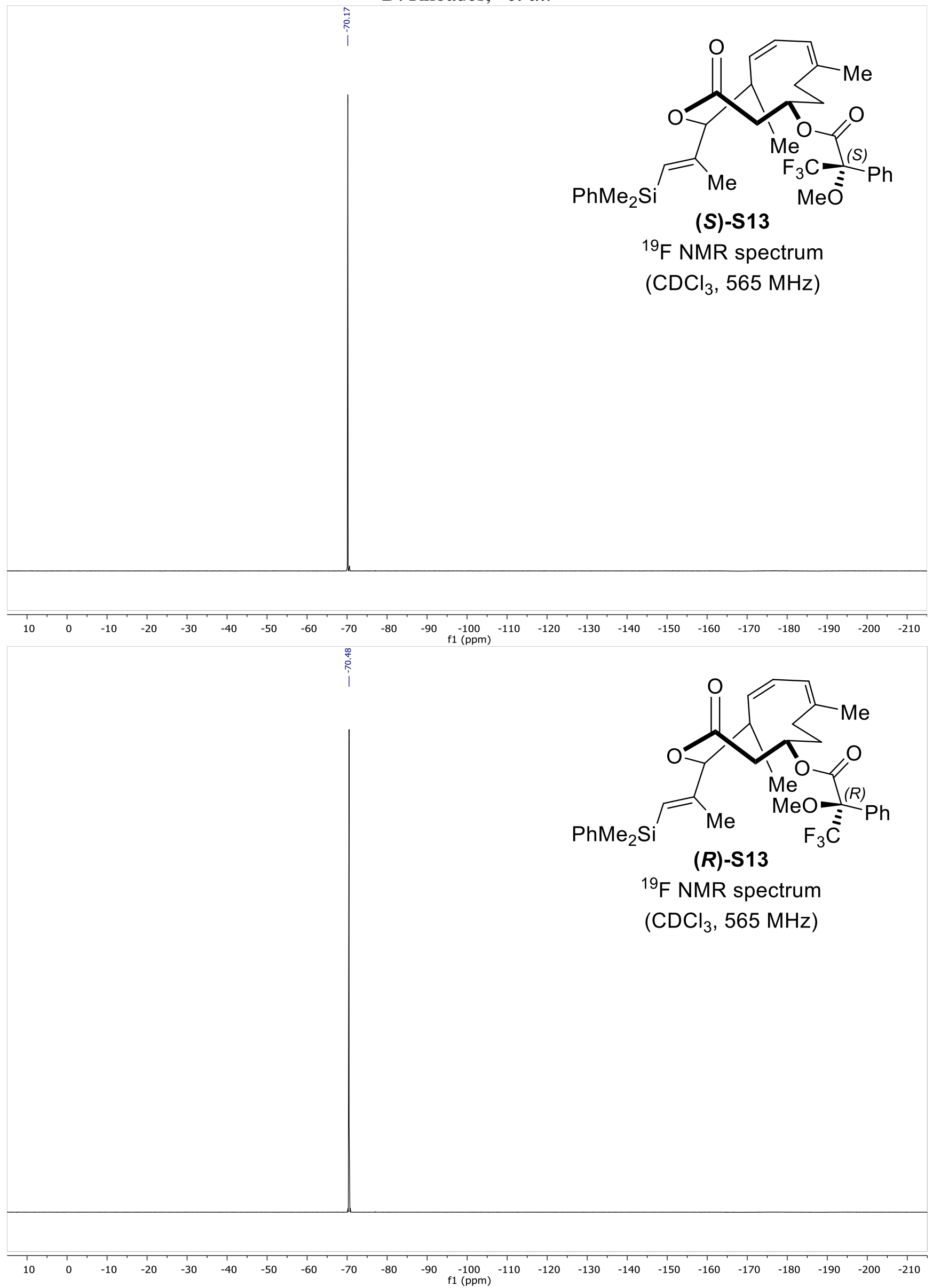
D. Rhoades, * et al.

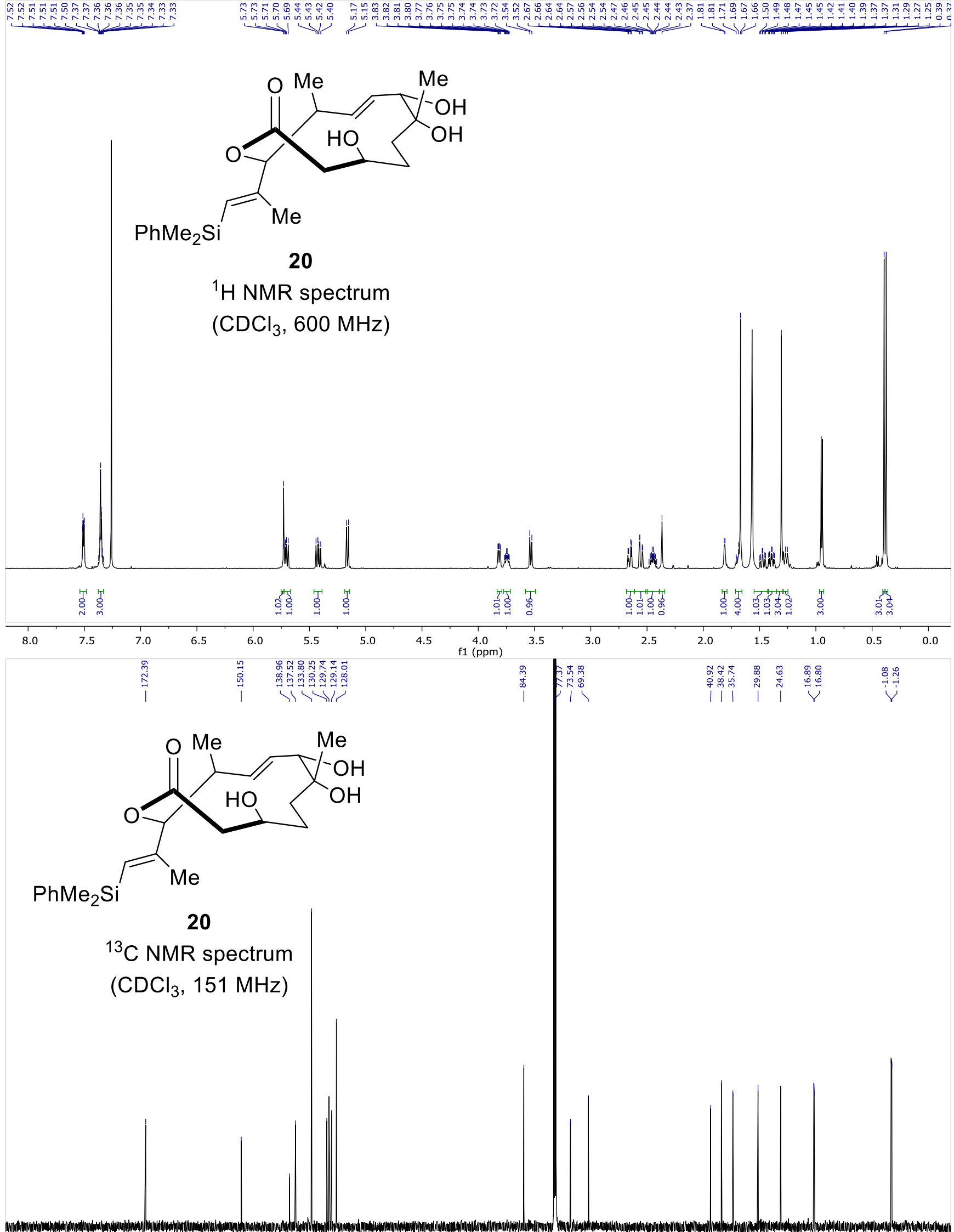

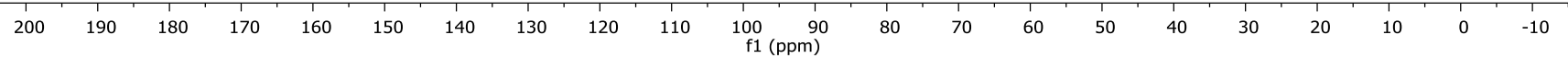


D. Rhoades, * et al.

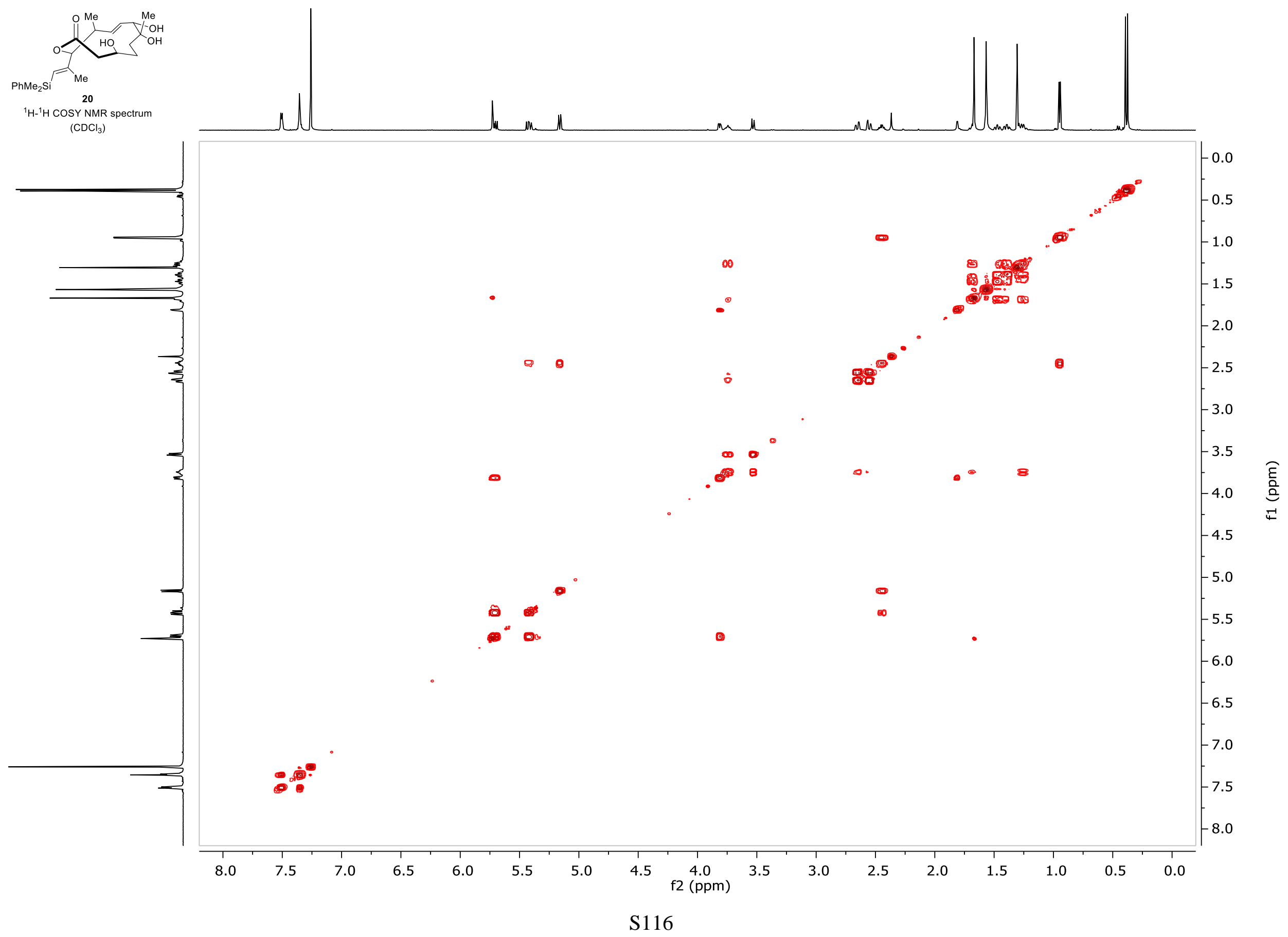


D. Rhoades, * et al.

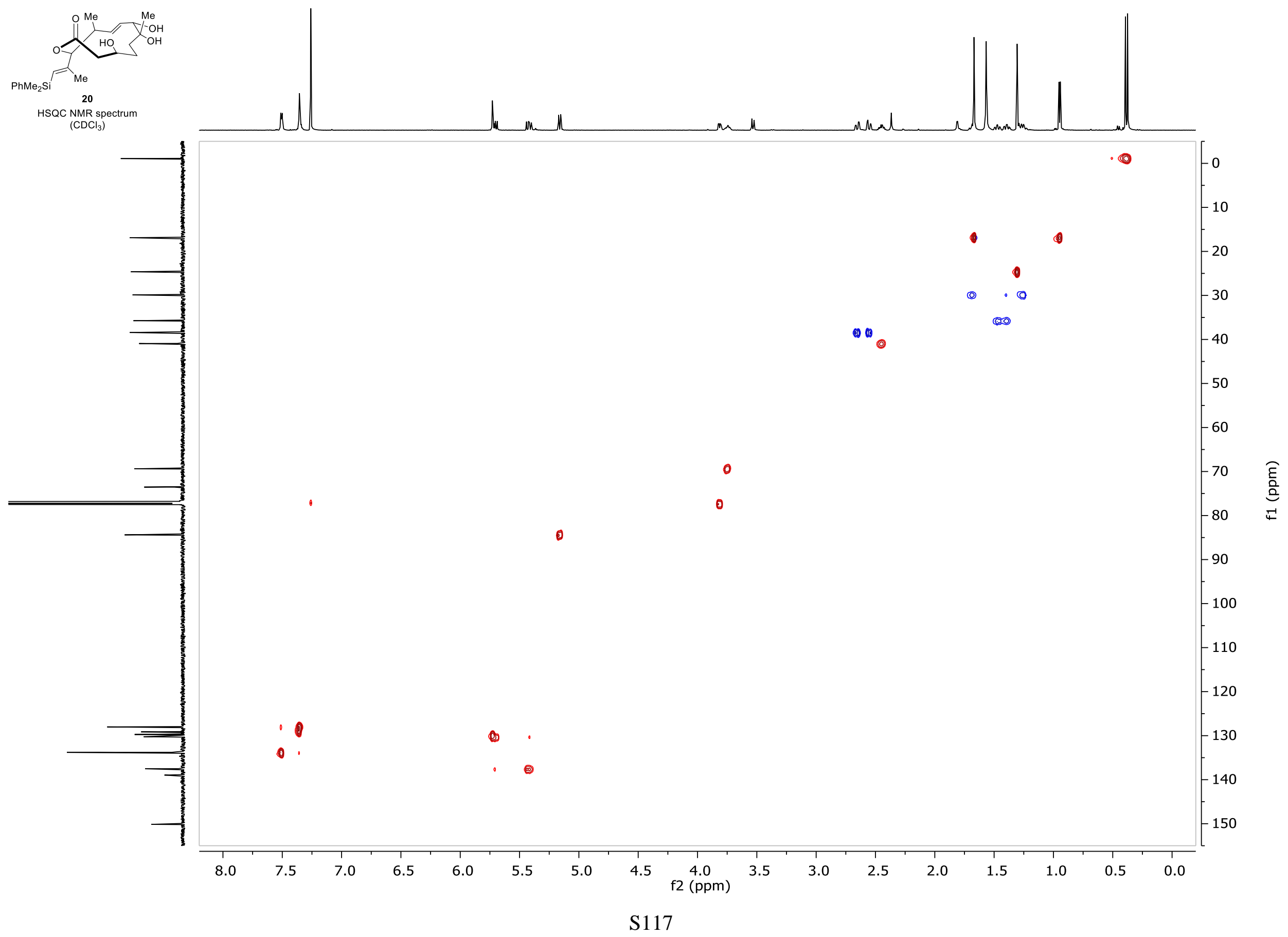




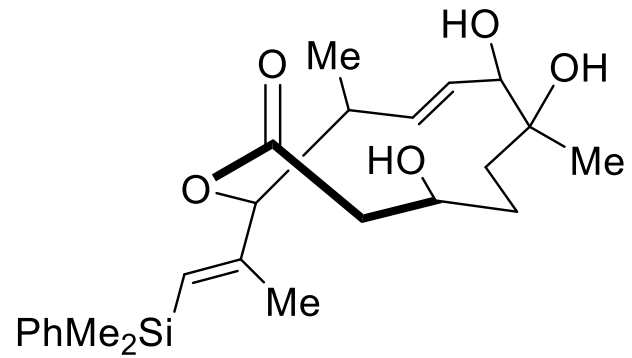

21

${ }^{1} \mathrm{H}$ NMR spectrum

$\left(\mathrm{CDCl}_{3}, 600 \mathrm{MHz}\right)$

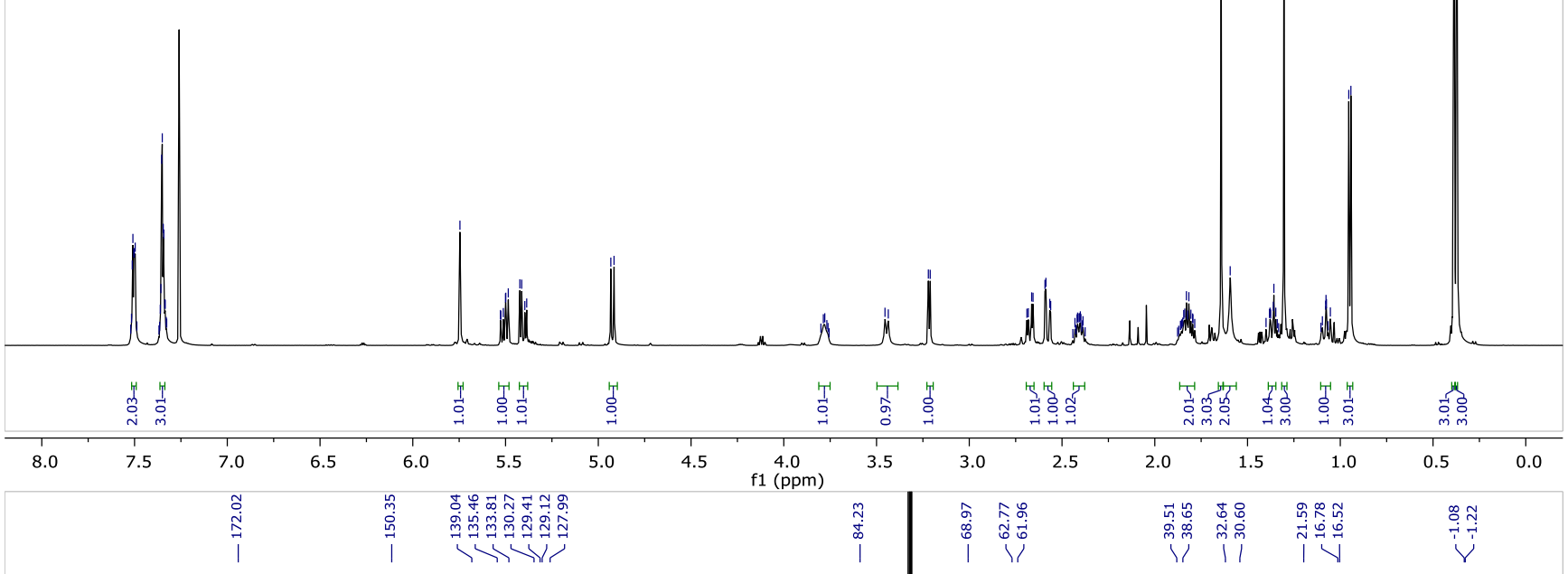

$\mathrm{PhMe}_{2} \mathrm{~S}$

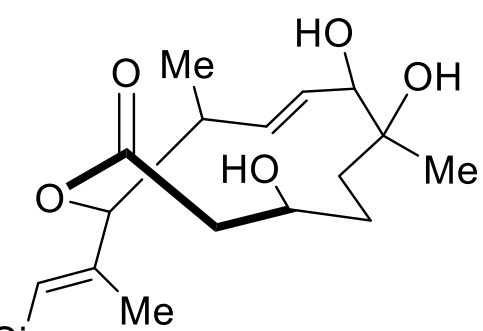

21

${ }^{13} \mathrm{C}$ NMR spectrum

$\left(\mathrm{CDCl}_{3}, 151 \mathrm{MHz}\right)$ 
D. Rhoades, * et al.

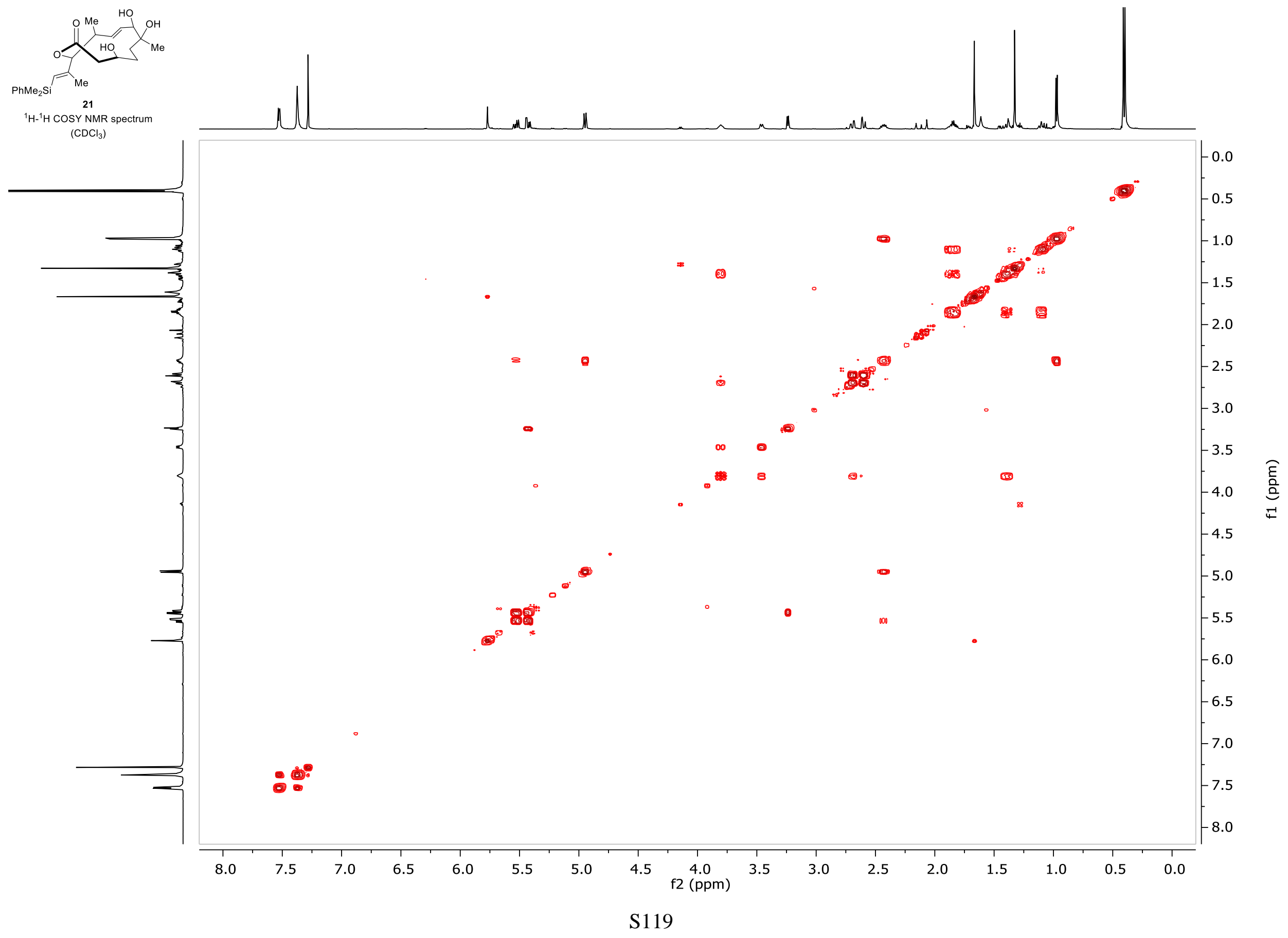


D. Rhoades, ${ }^{*}$ et al.

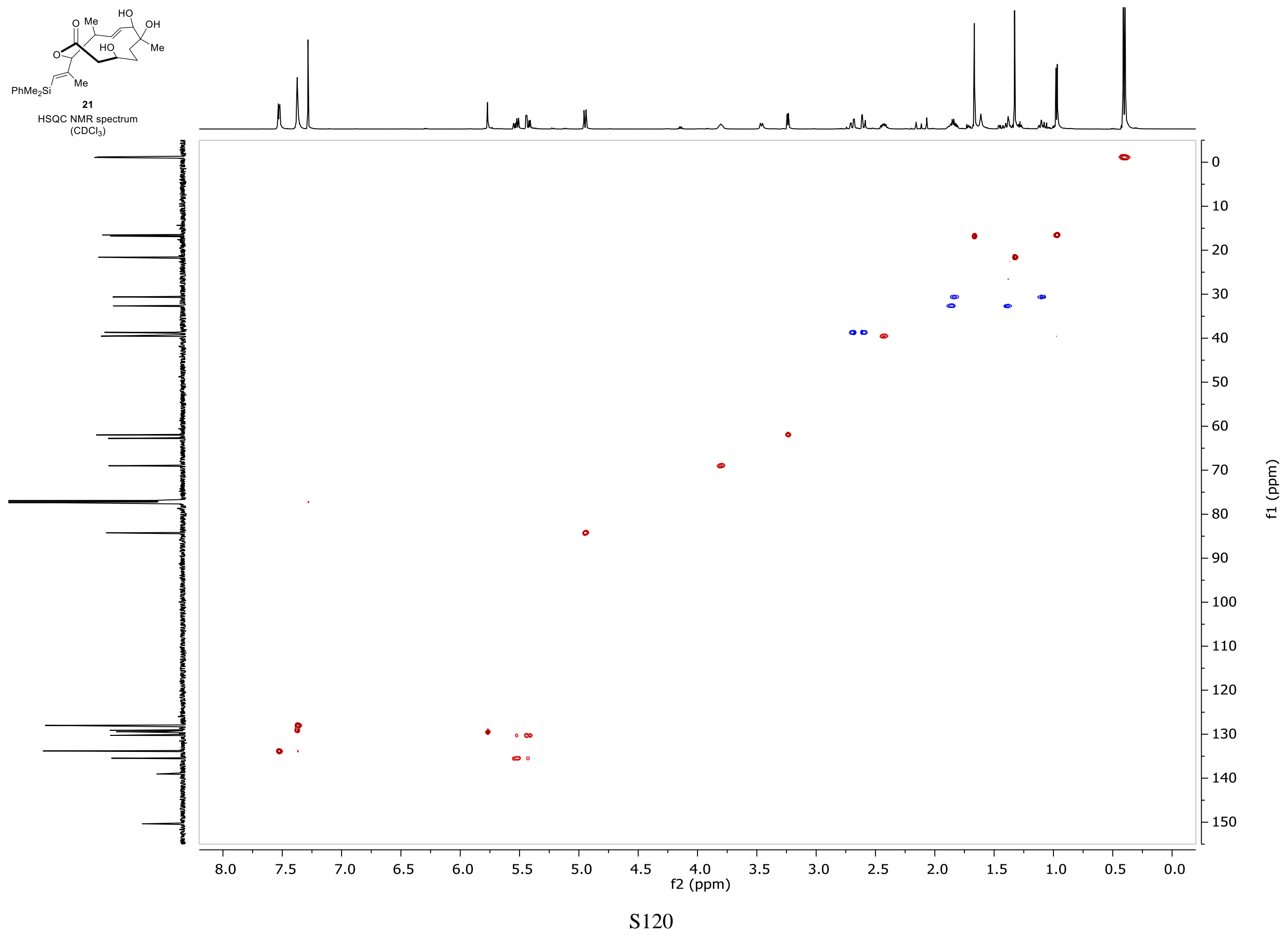




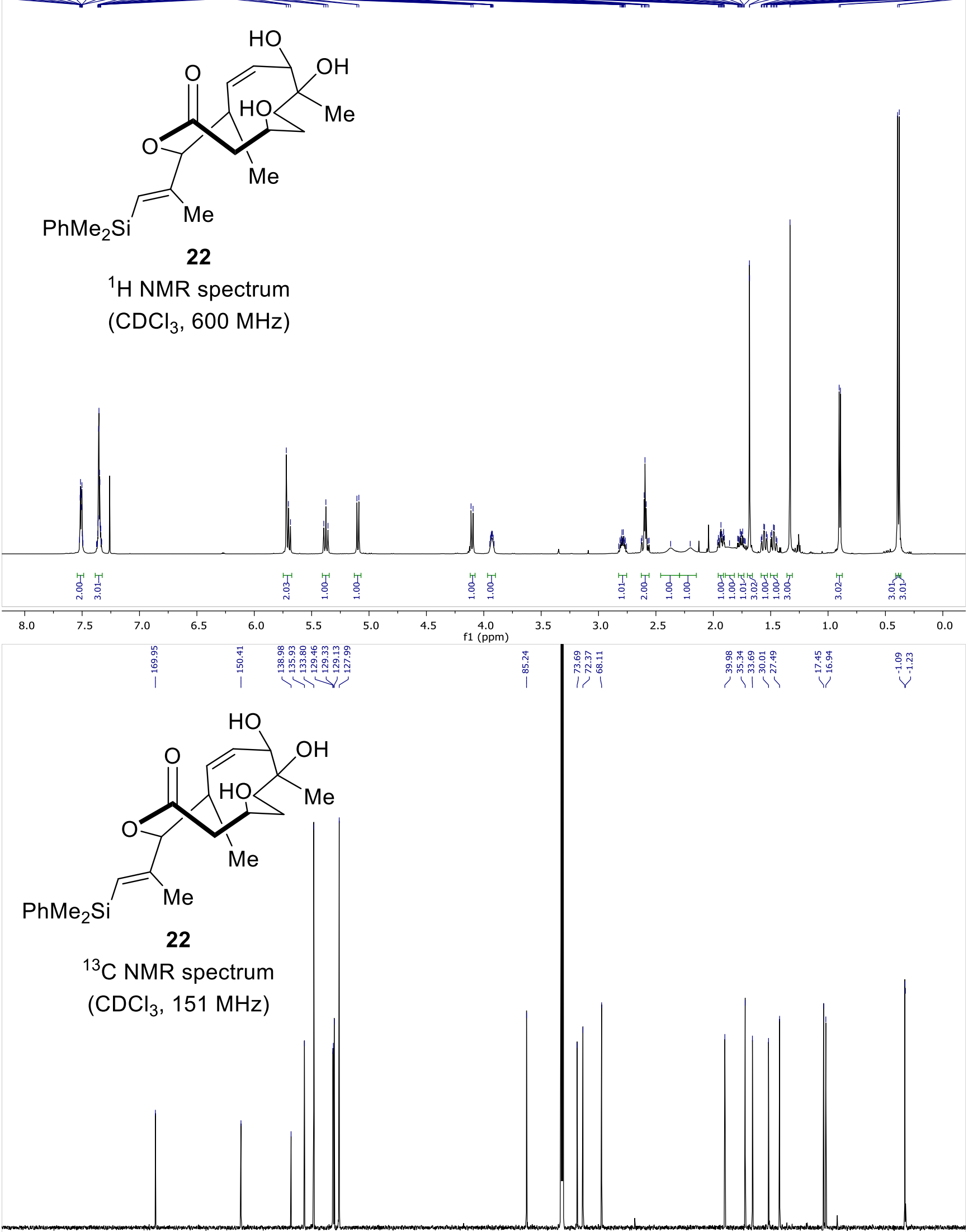


D. Rhoades, * et al.

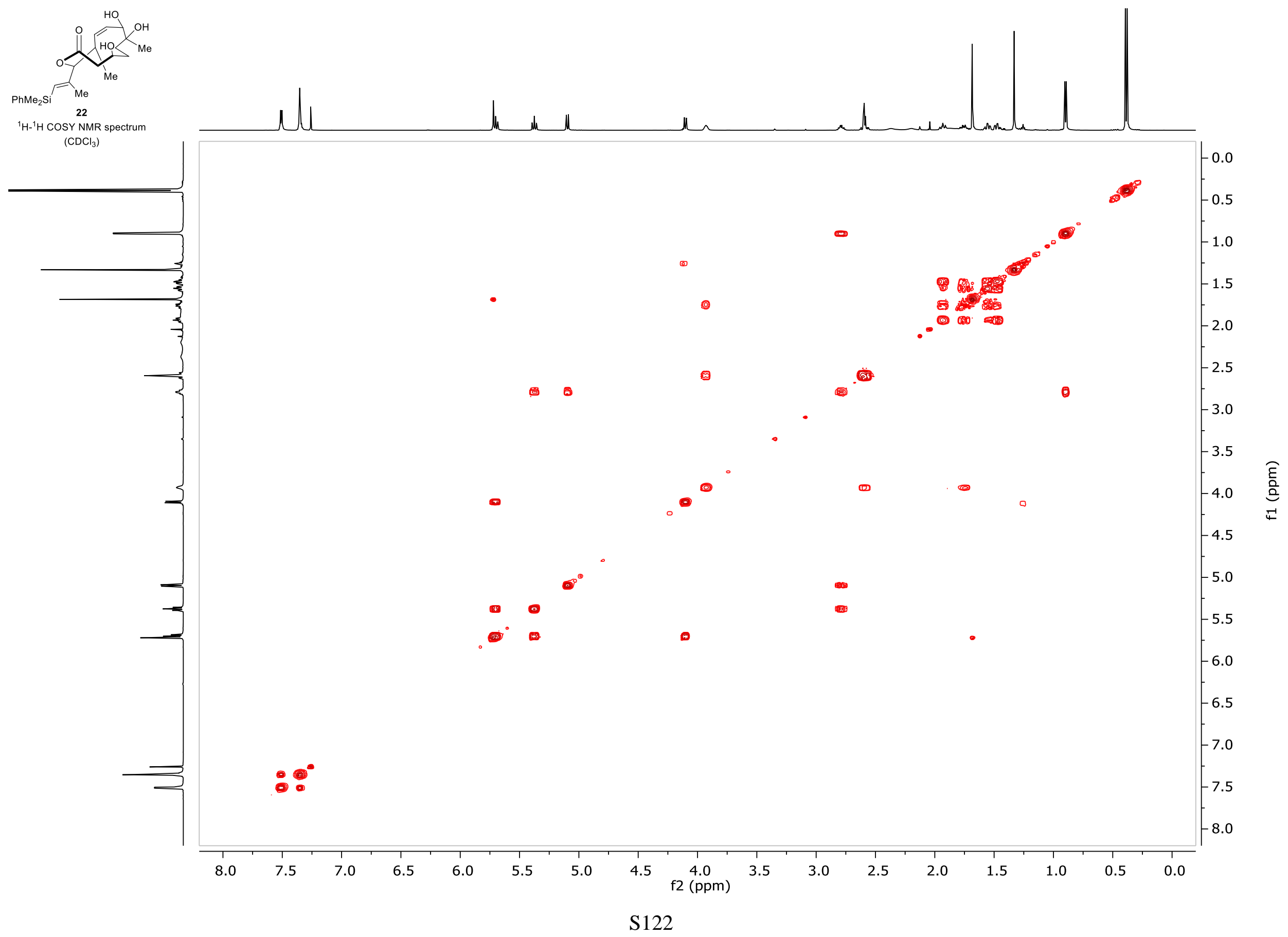


D. Rhoades, ${ }^{*}$ et al.

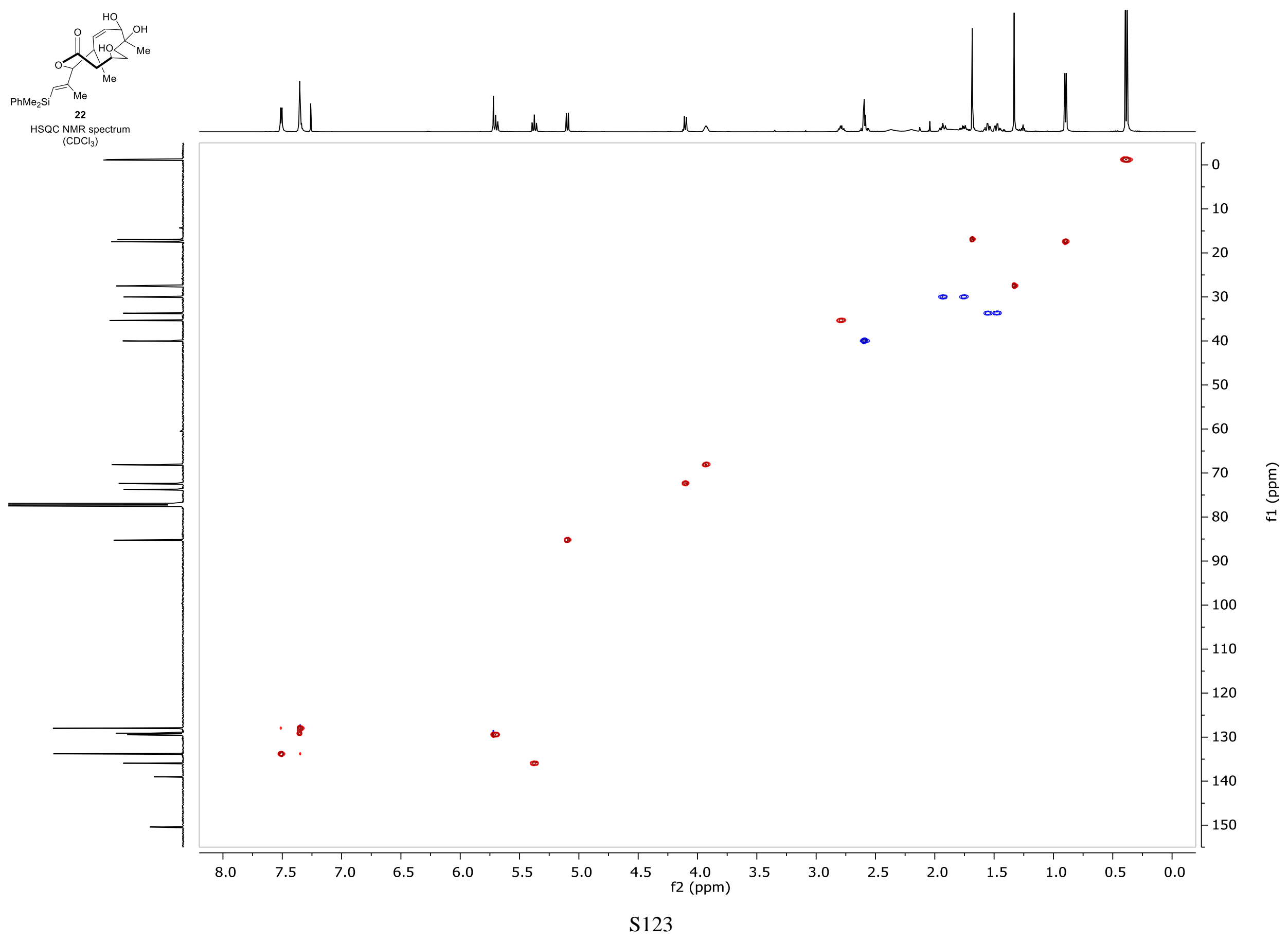


D. Rhoades, * et al.

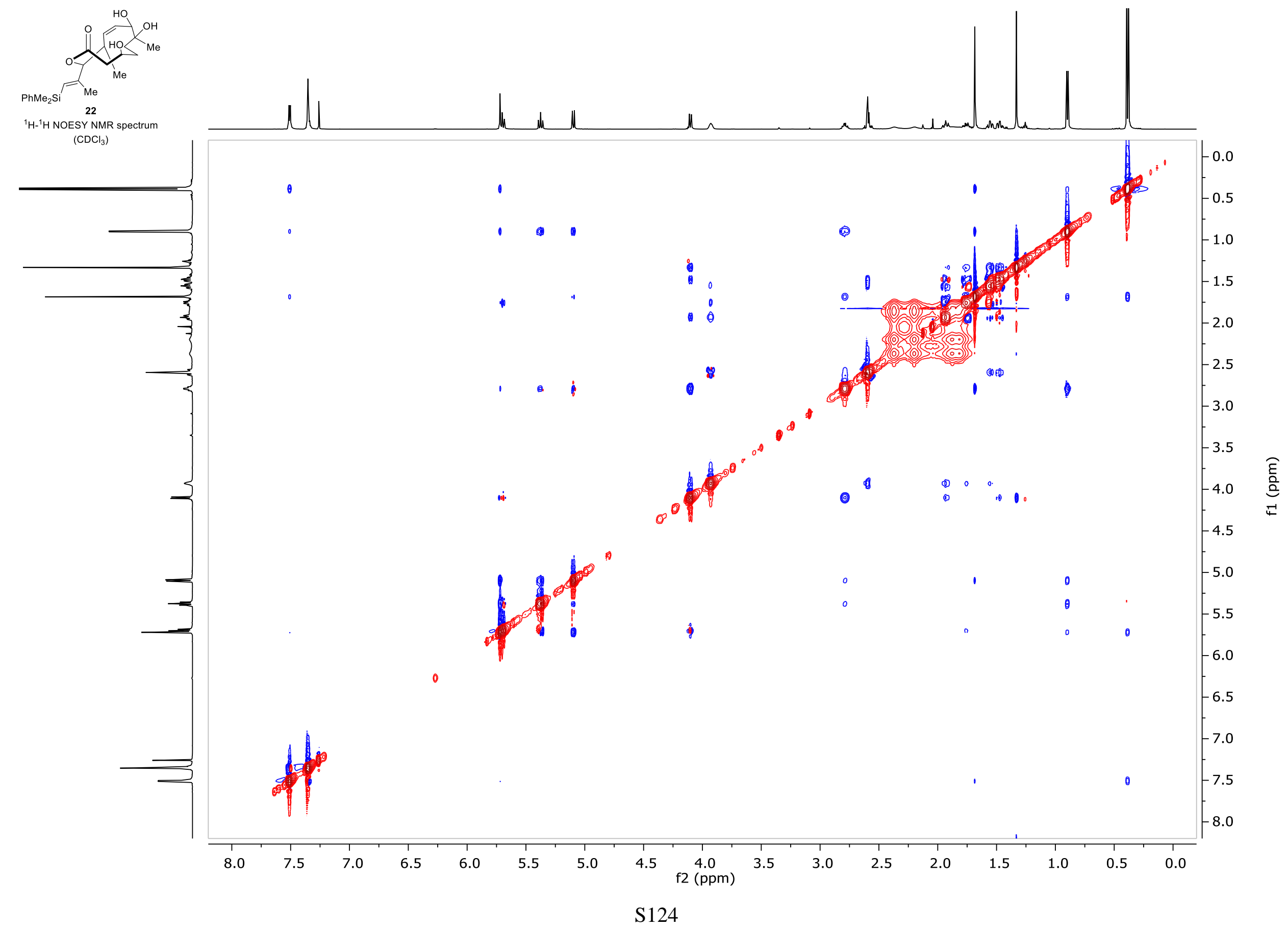


D. Rhoades, * et al.

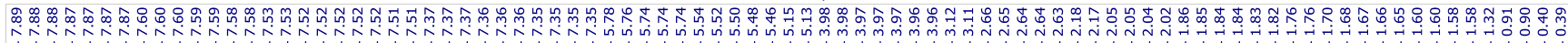

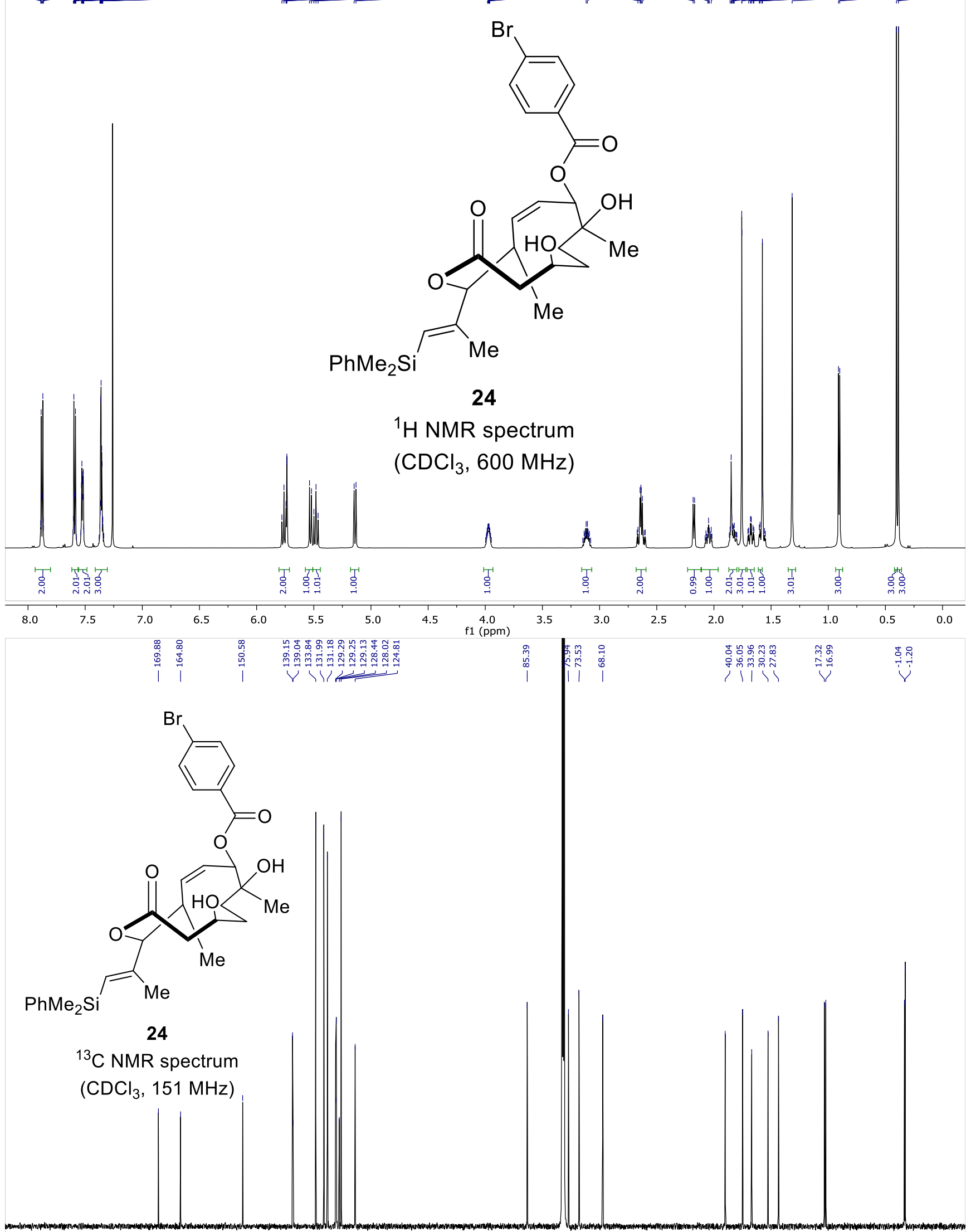


D. Rhoades, * et al.

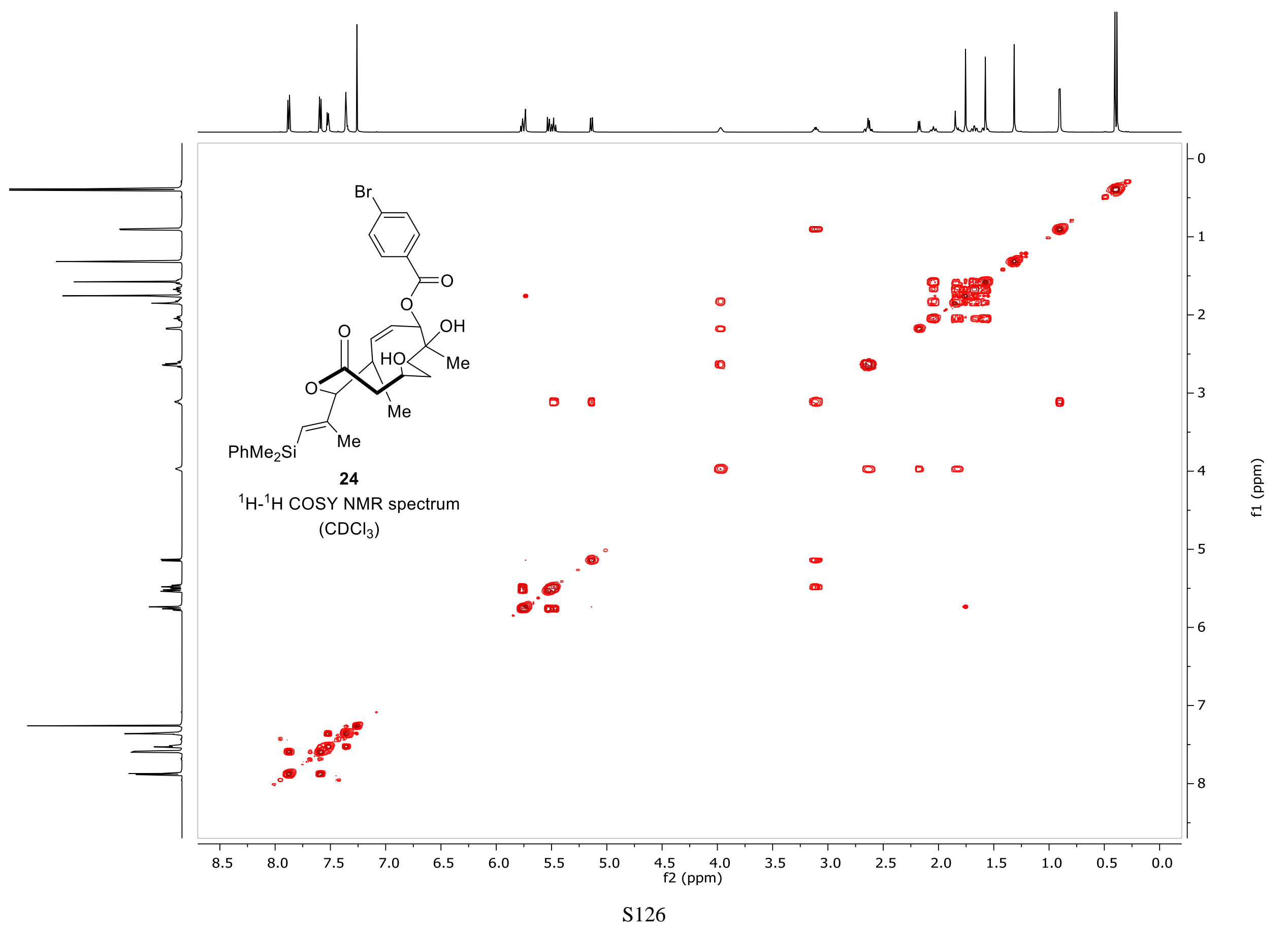


D. Rhoades, * et al.

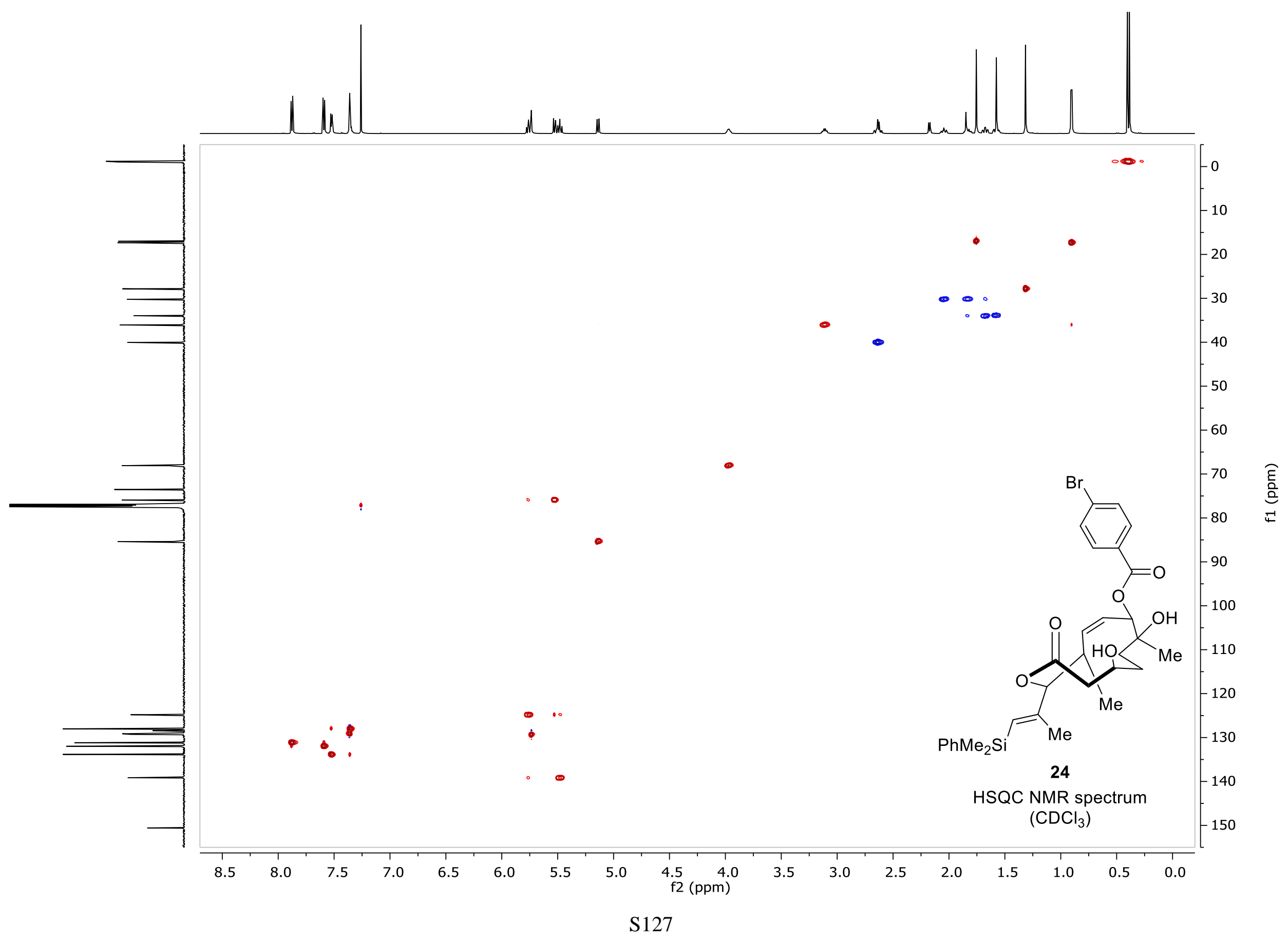




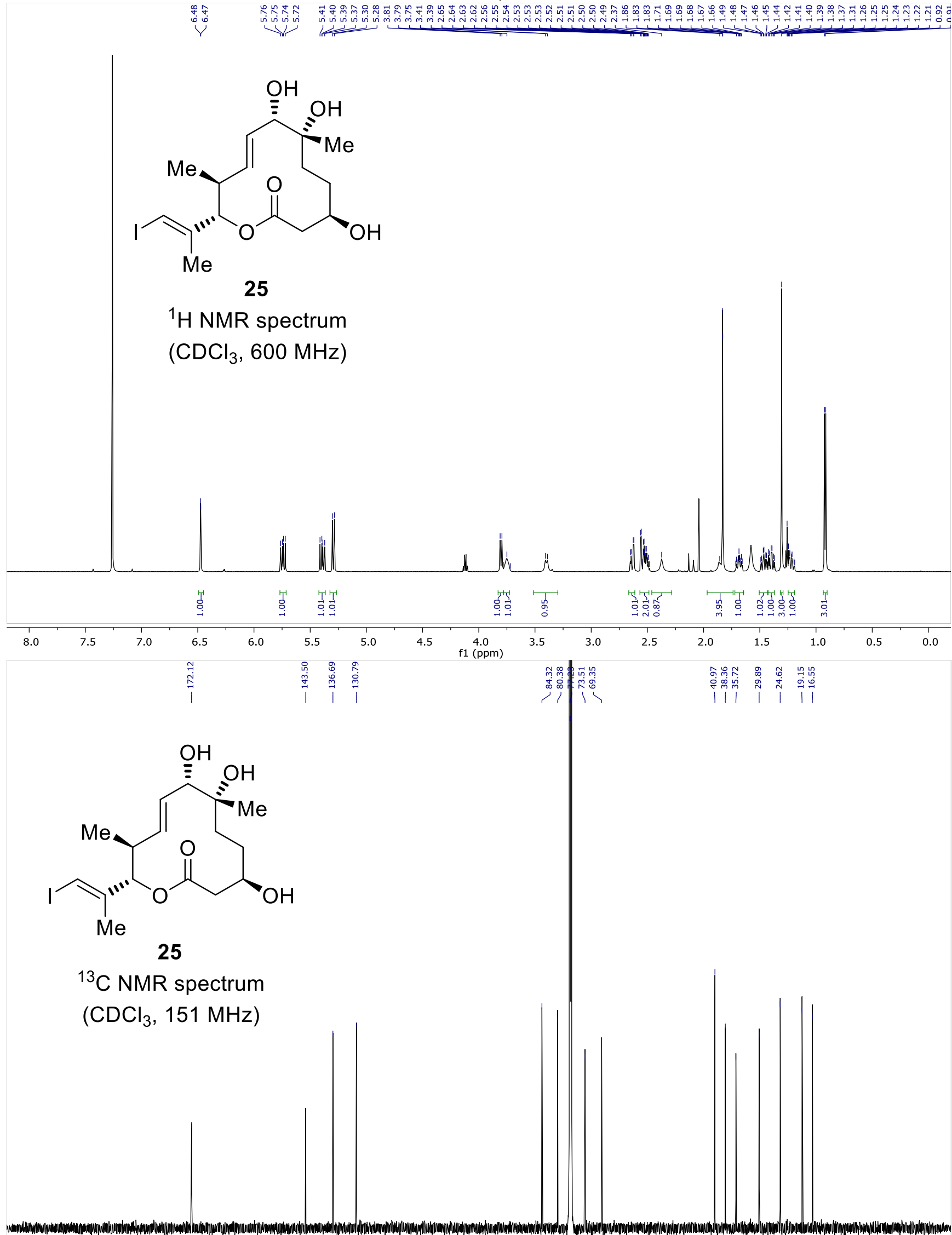

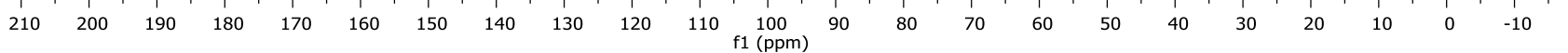




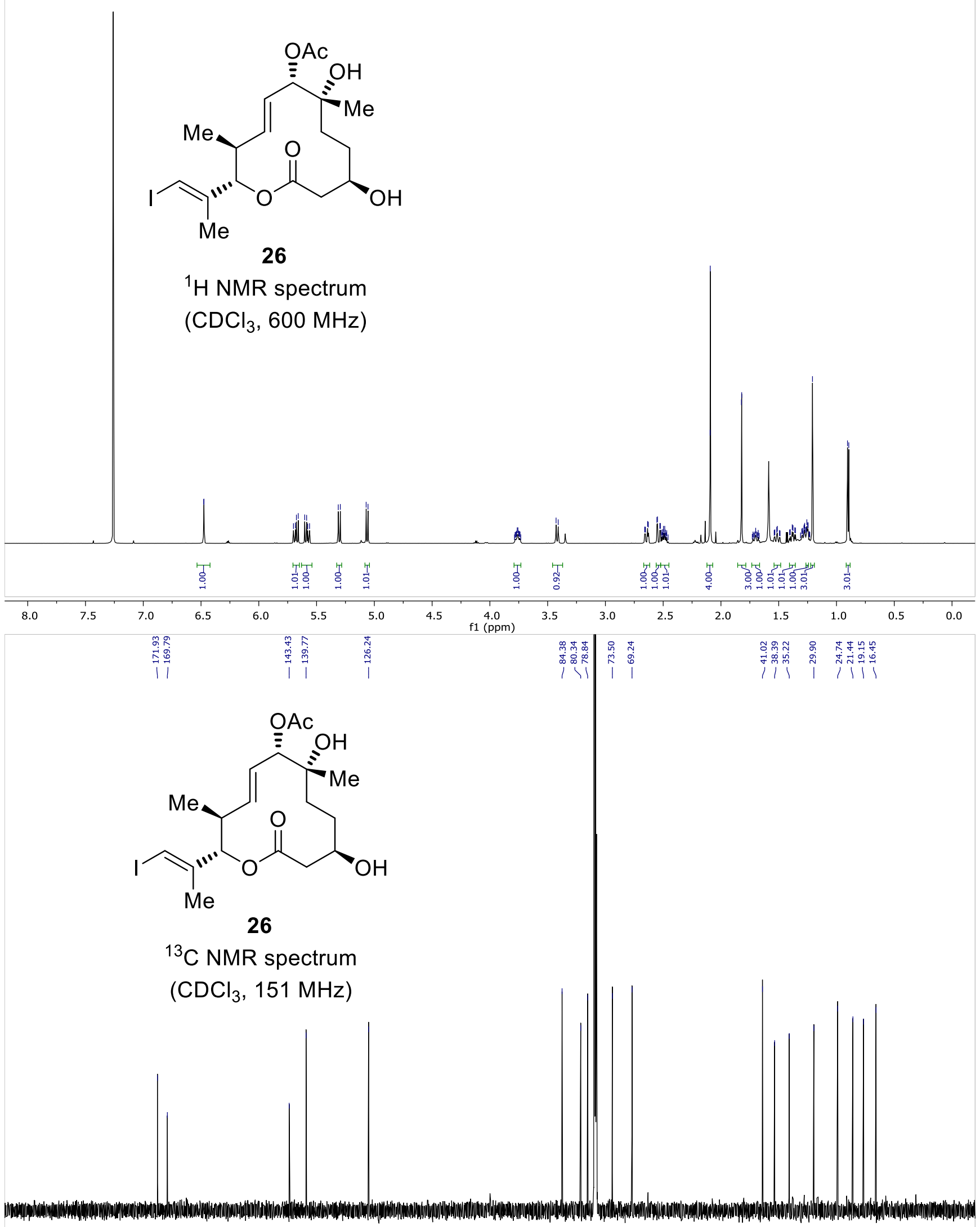


D. Rhoades, * et al.

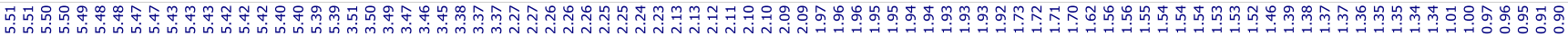

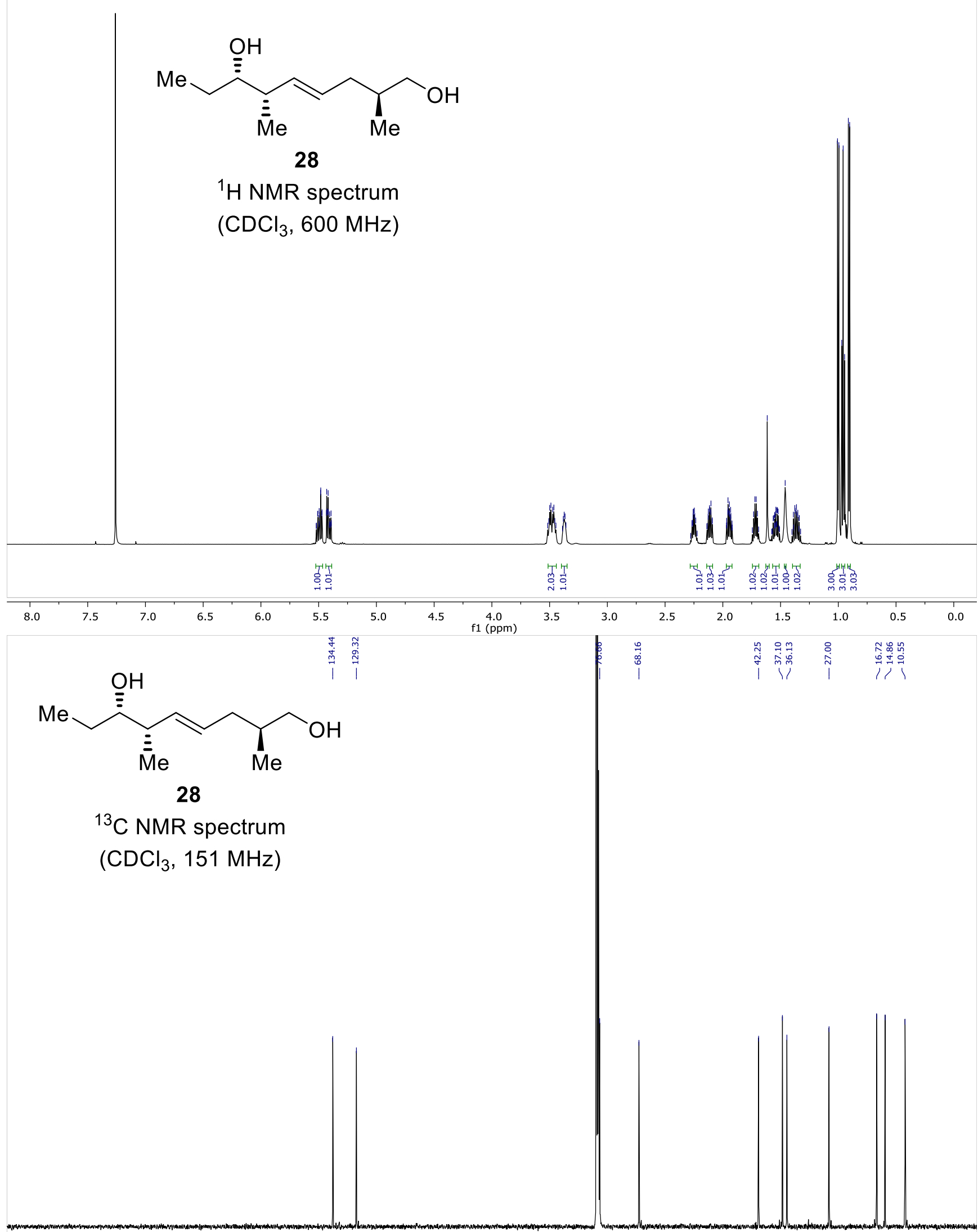


D. Rhoades, * et al.

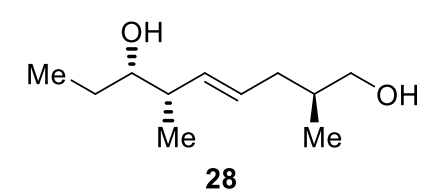

${ }^{1} \mathrm{H}-{ }^{1} \mathrm{H}$ COSY NMR spectrum

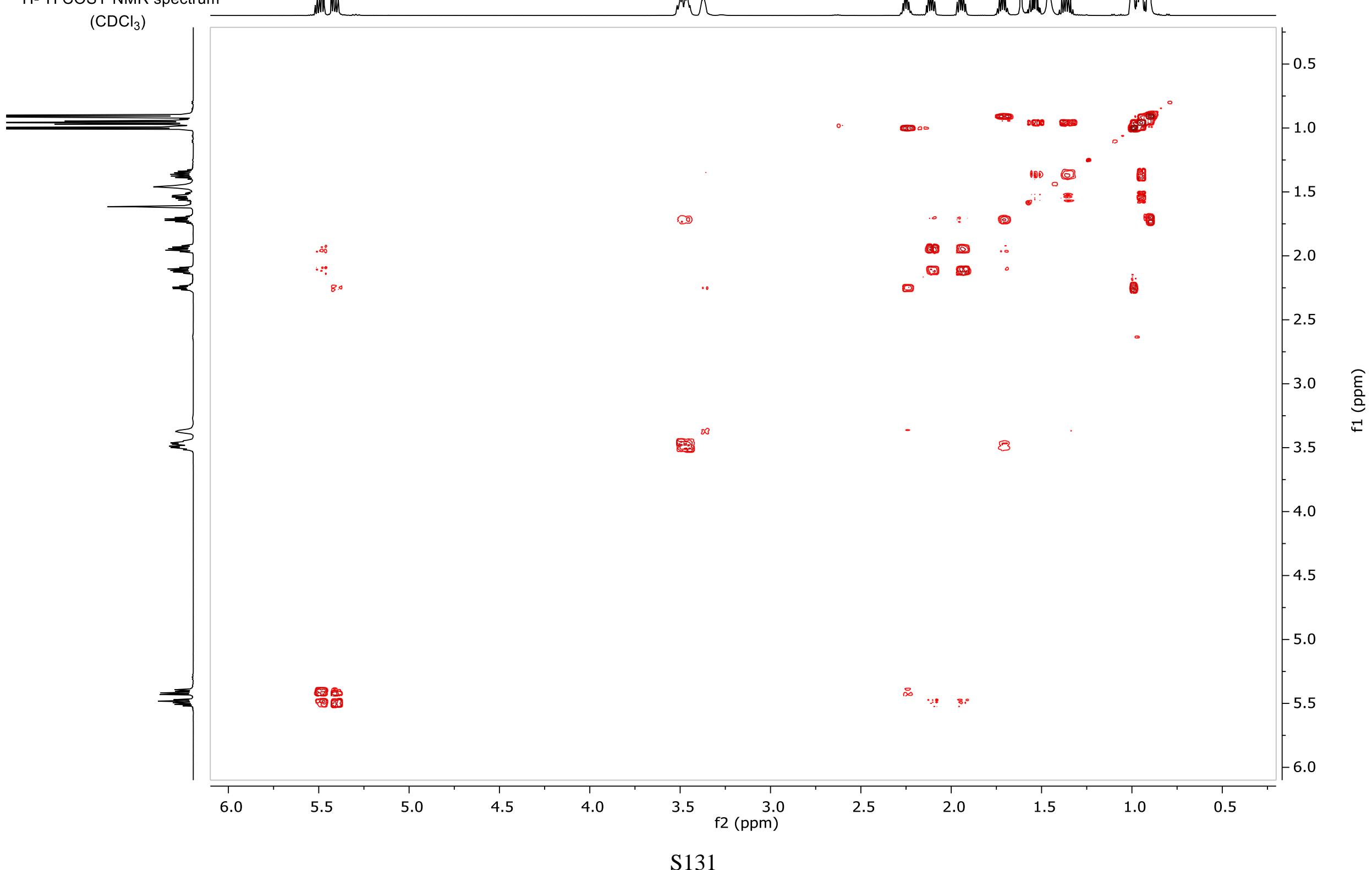

S131 
D. Rhoades, * et al.

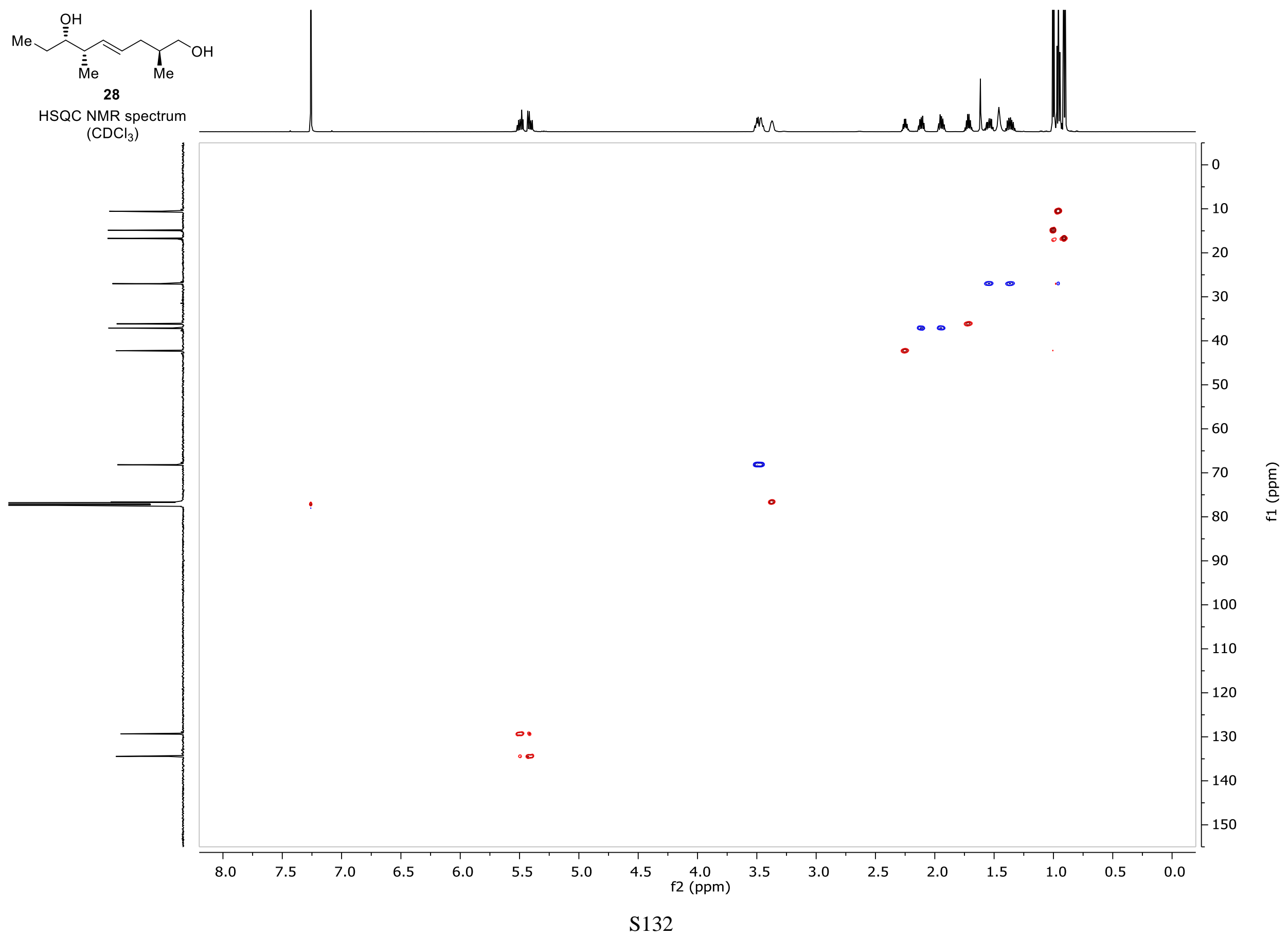


D. Rhoades, * et al.

莺<smiles>CC[C@H](O)[C@H](C)[C@H]1O[C@@H]1C[C@H](C)CO</smiles>

30

${ }^{1} \mathrm{H}$ NMR spectrum

$\left(\mathrm{CDCl}_{3}, 600 \mathrm{MHz}\right)$
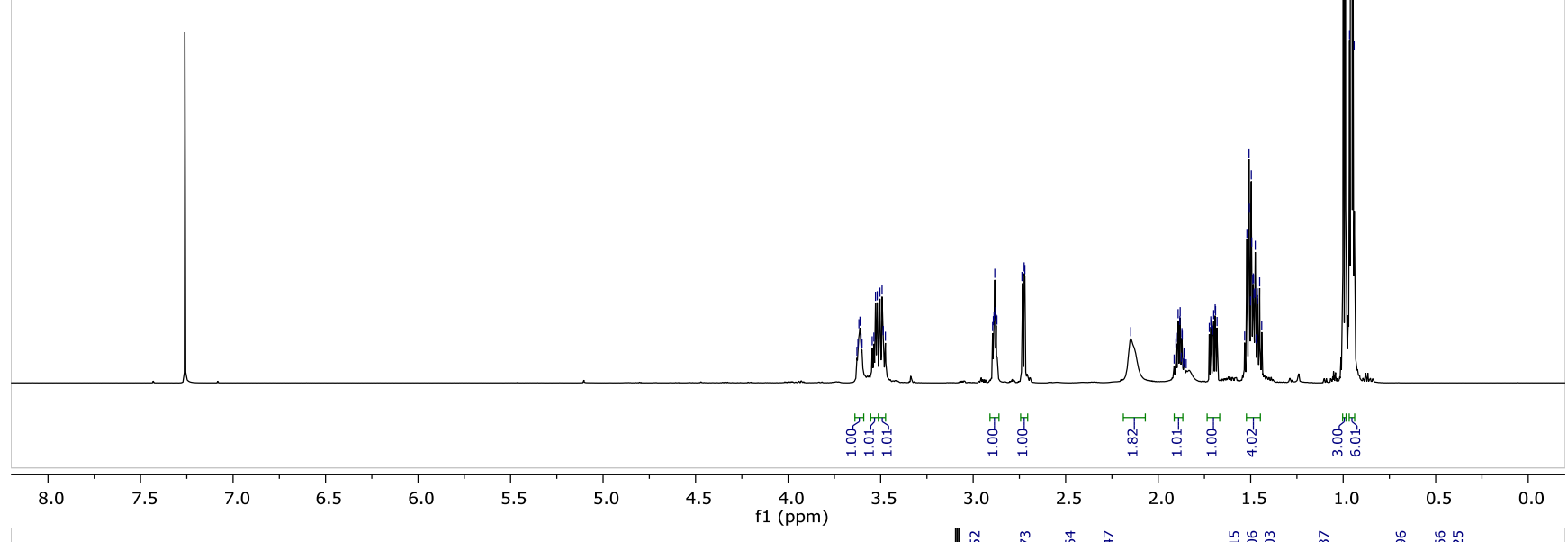<smiles>CC[C@H](O)[C@H](C)[C@H]1O[C@@H]1C[C@H](C)CO</smiles>

${ }^{13} \mathrm{C}$ NMR spectrum

$\left(\mathrm{CDCl}_{3}, 151 \mathrm{MHz}\right)$

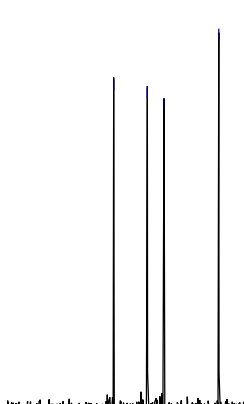


D. Rhoades, * et al.

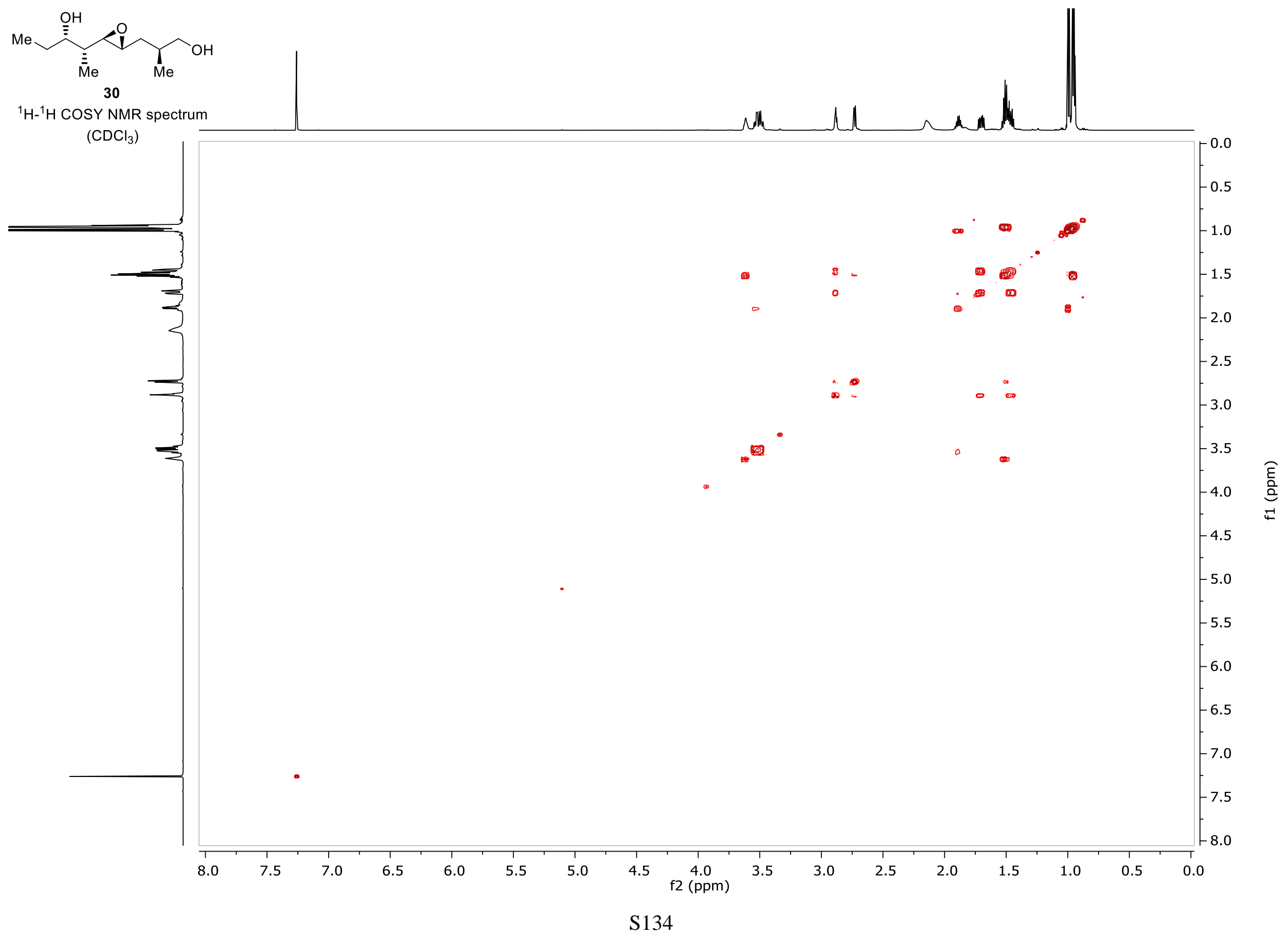


D. Rhoades, * et al.

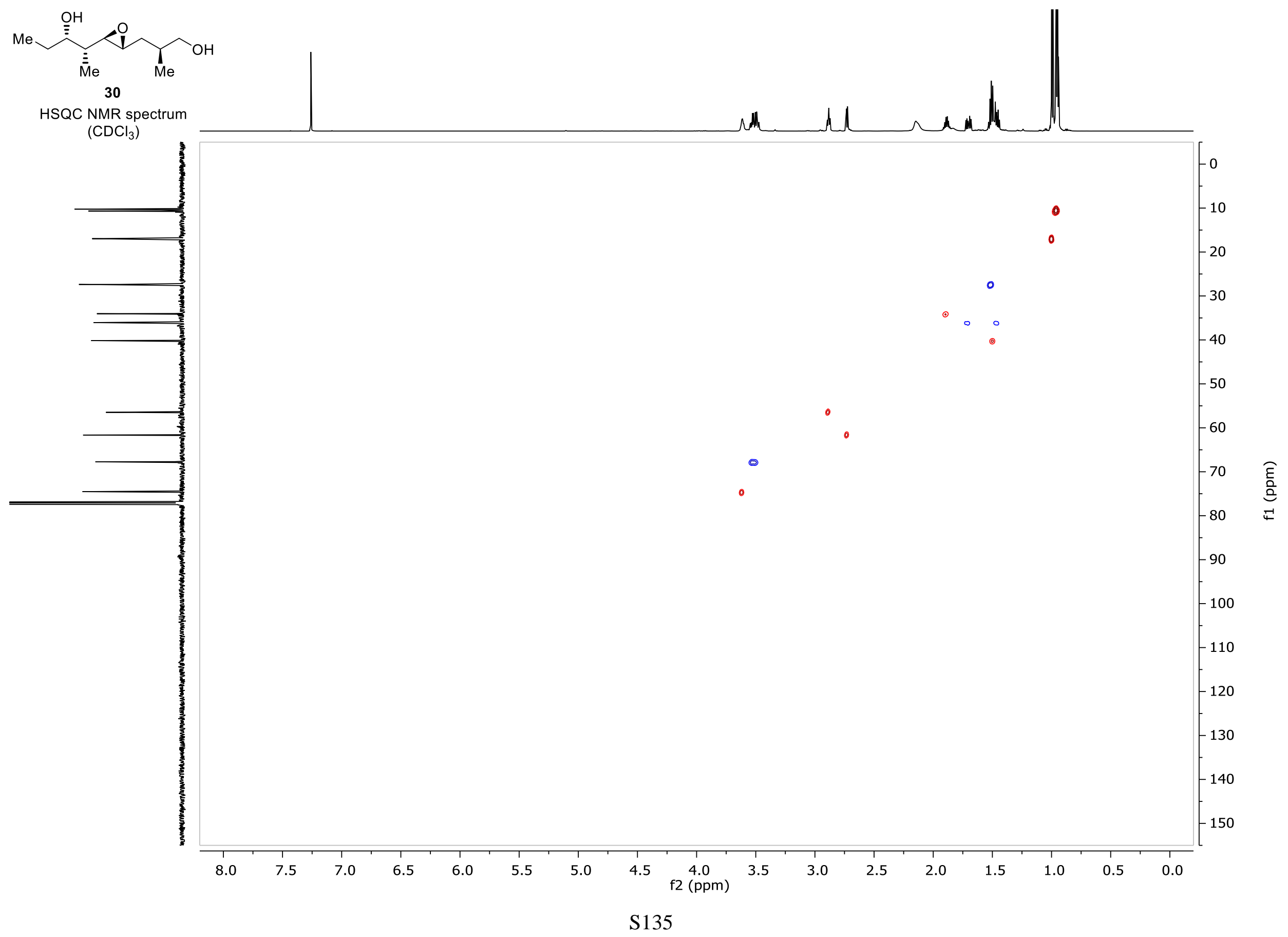


<smiles>CC[C@H](O)[C@H](C)[C@H]1O[C@@H]1C[C@H](C)C=O</smiles>

31

${ }^{1} \mathrm{H}$ NMR spectrum $\left(\mathrm{CDCl}_{3}, 600 \mathrm{MHz}\right)$
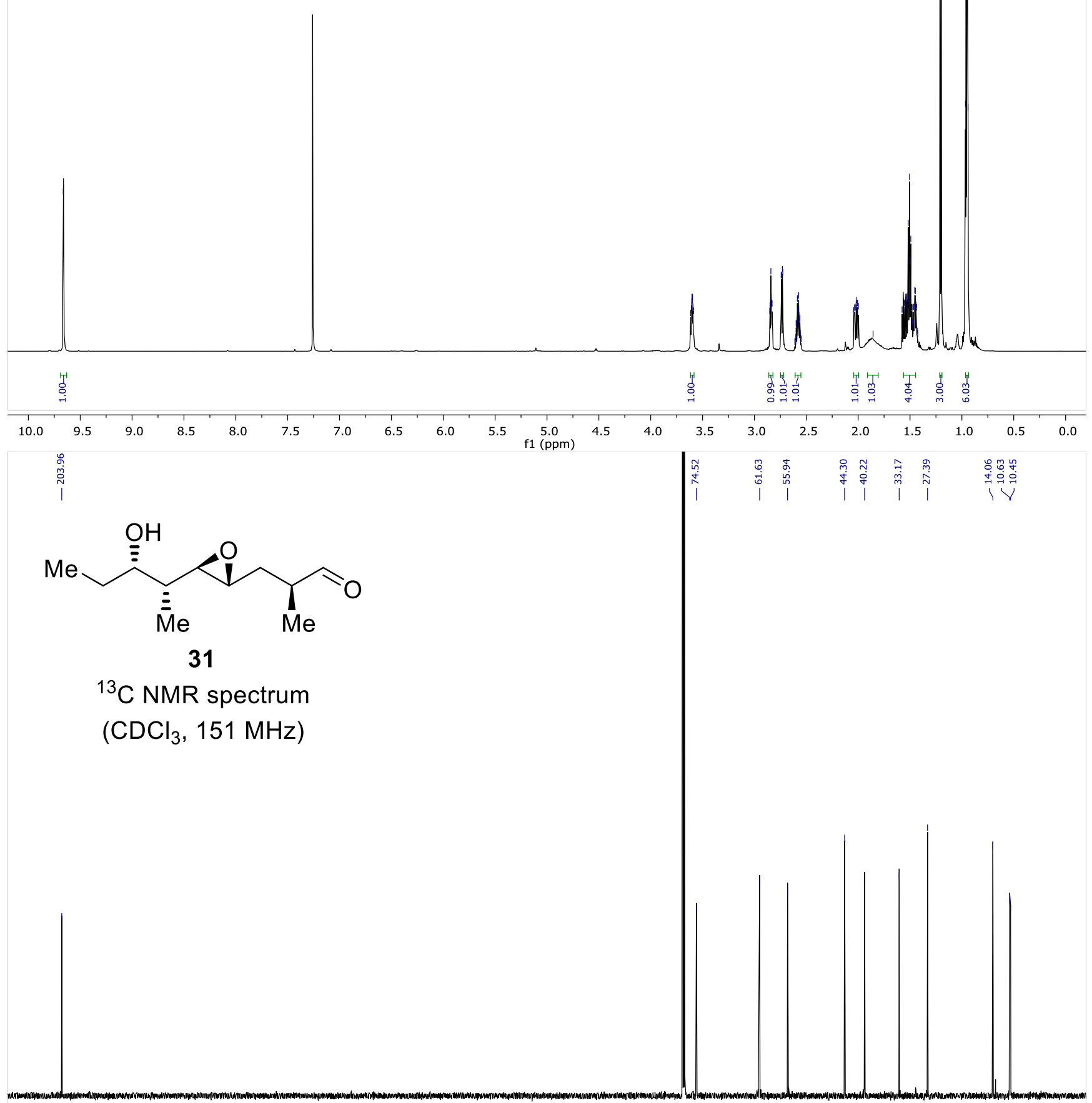
D. Rhoades, * et al.

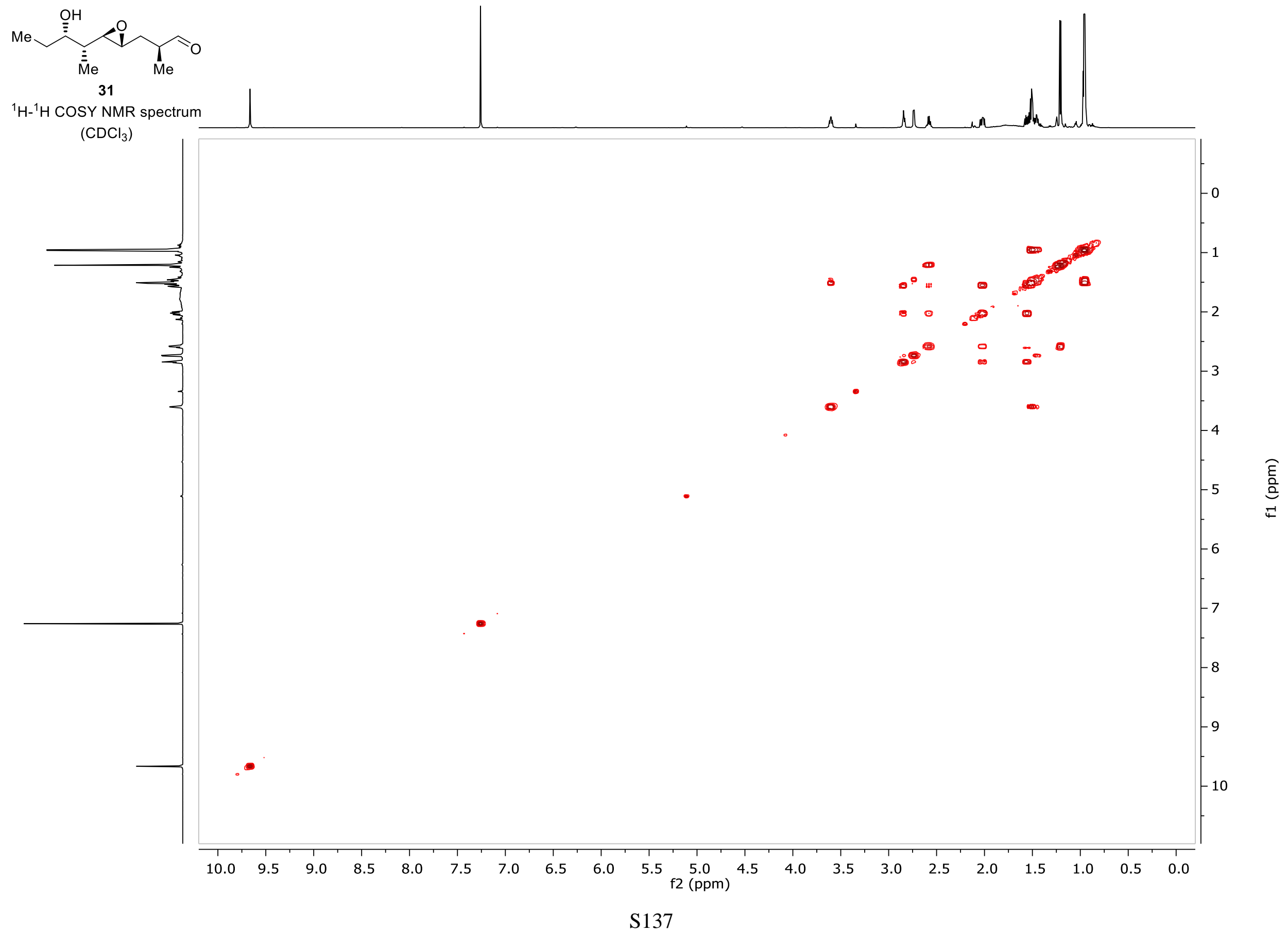


D. Rhoades, * et al.

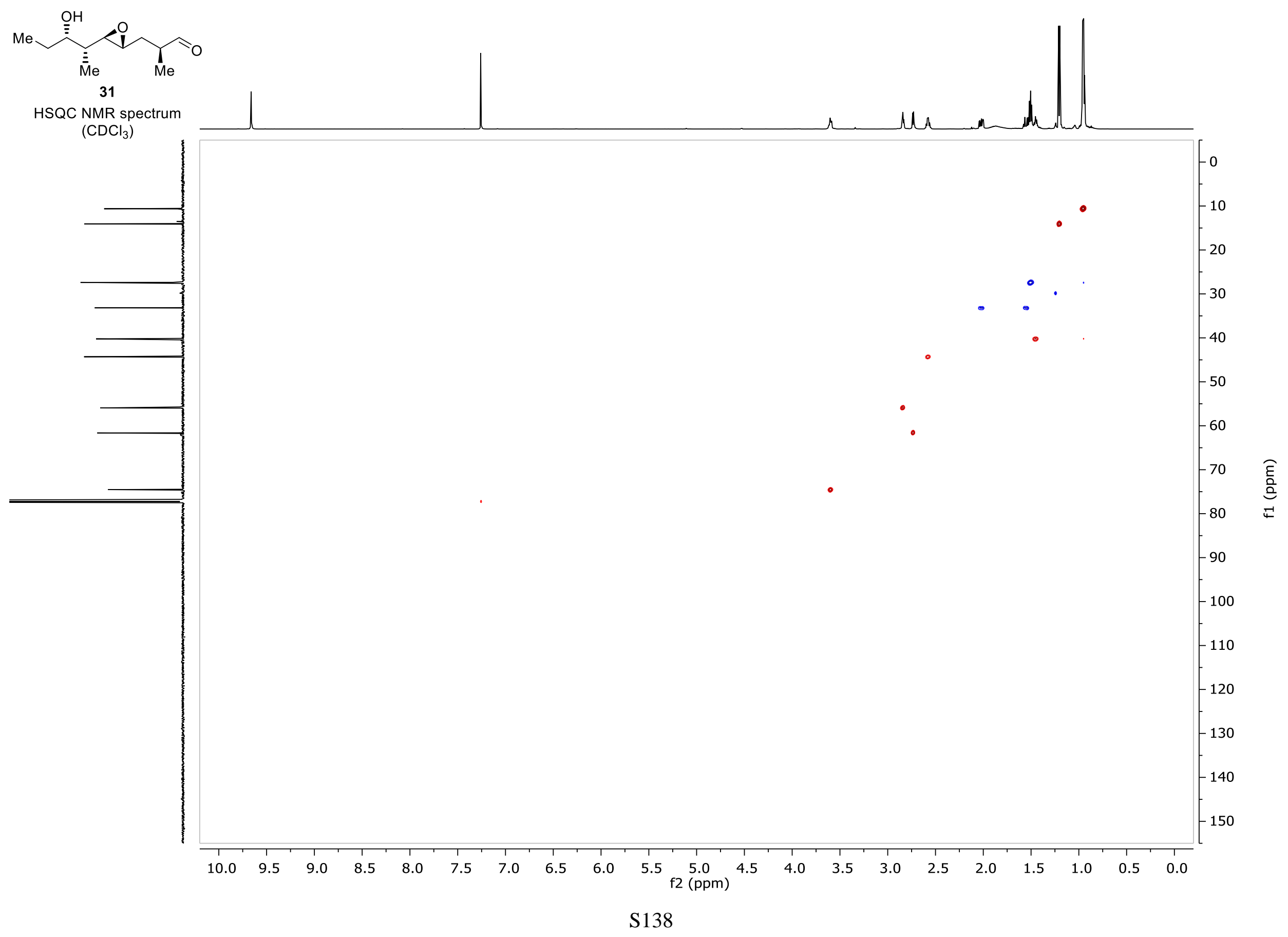


D. Rhoades, * et al.

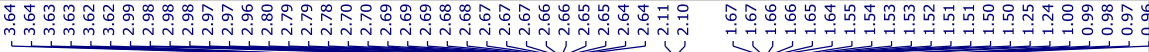<smiles>C#C[C@H](C)C[C@@H]1O[C@H]1[C@H](C)[C@H](O)CC</smiles>

33

${ }^{1} \mathrm{H}$ NMR spectrum

$\left(\mathrm{CDCl}_{3}, 600 \mathrm{MHz}\right)$
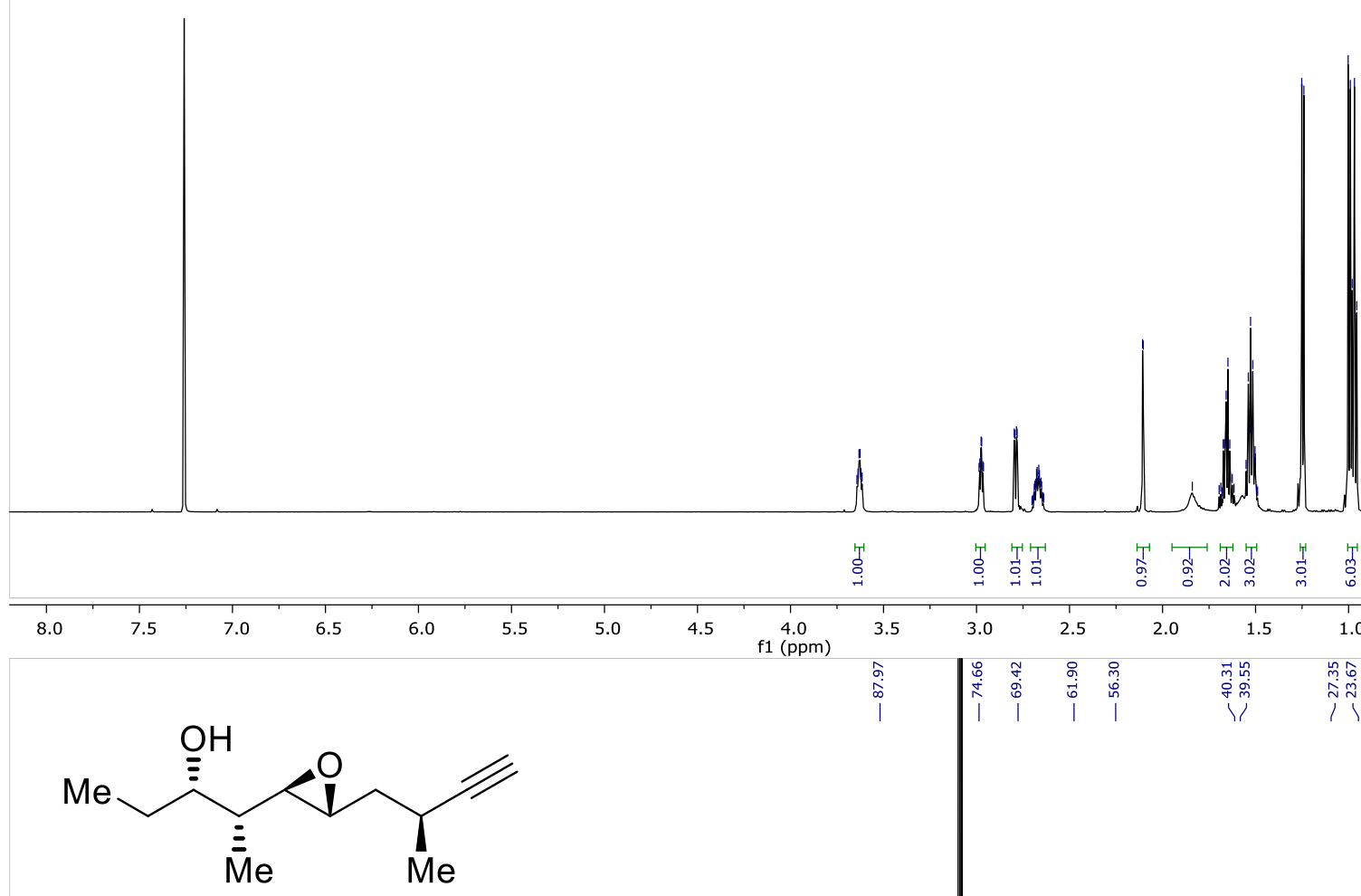

33

${ }^{13} \mathrm{C}$ NMR spectrum

$\left(\mathrm{CDCl}_{3}, 151 \mathrm{MHz}\right)$

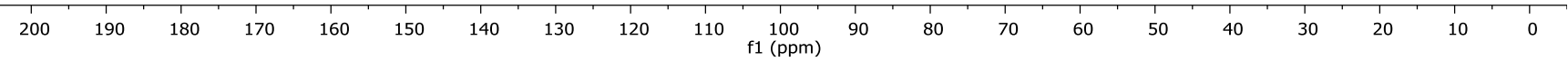


D. Rhoades,* et al.

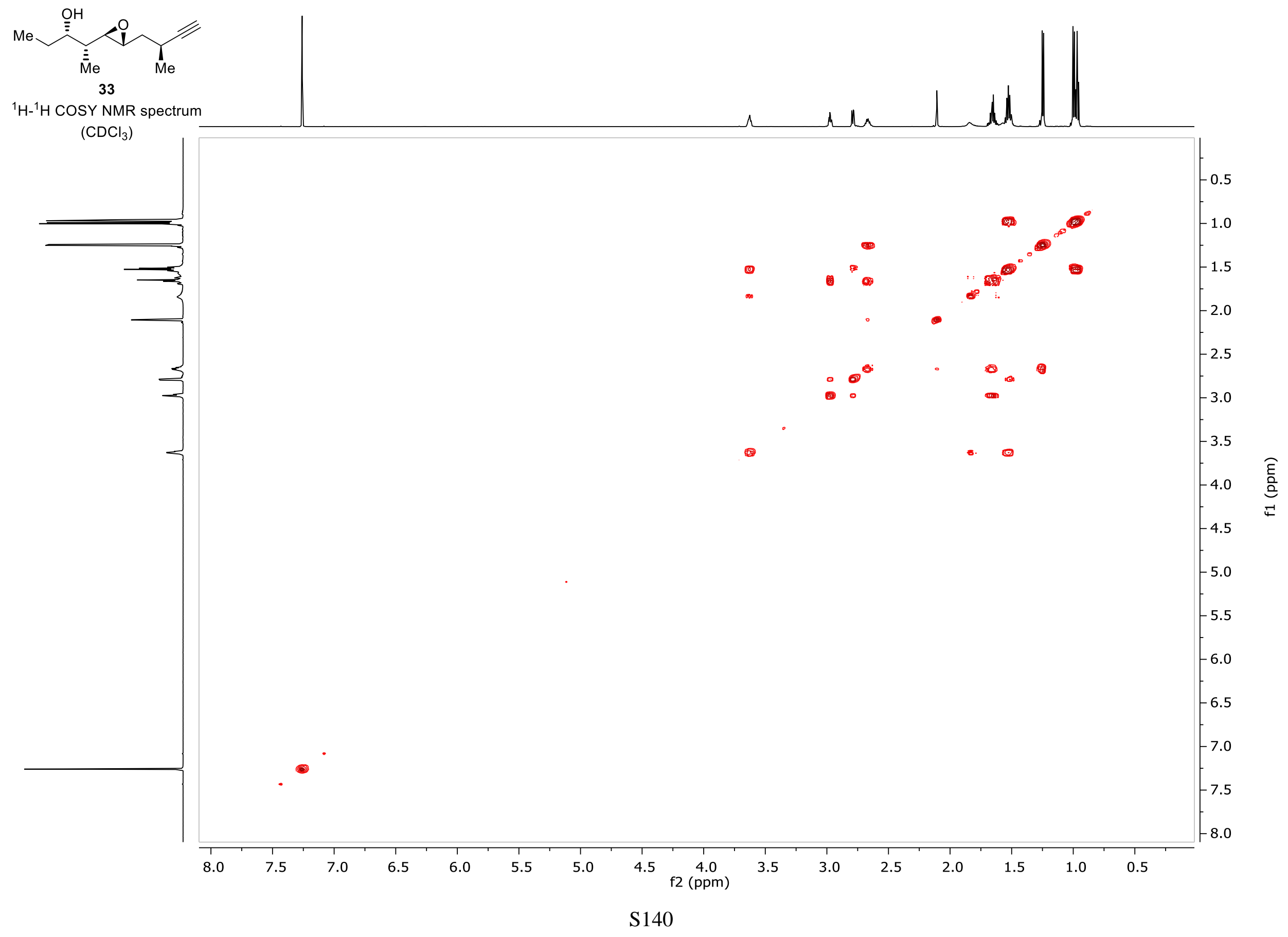


D. Rhoades, * et al.

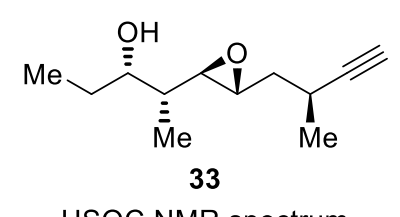

HSQC NMR spectrum

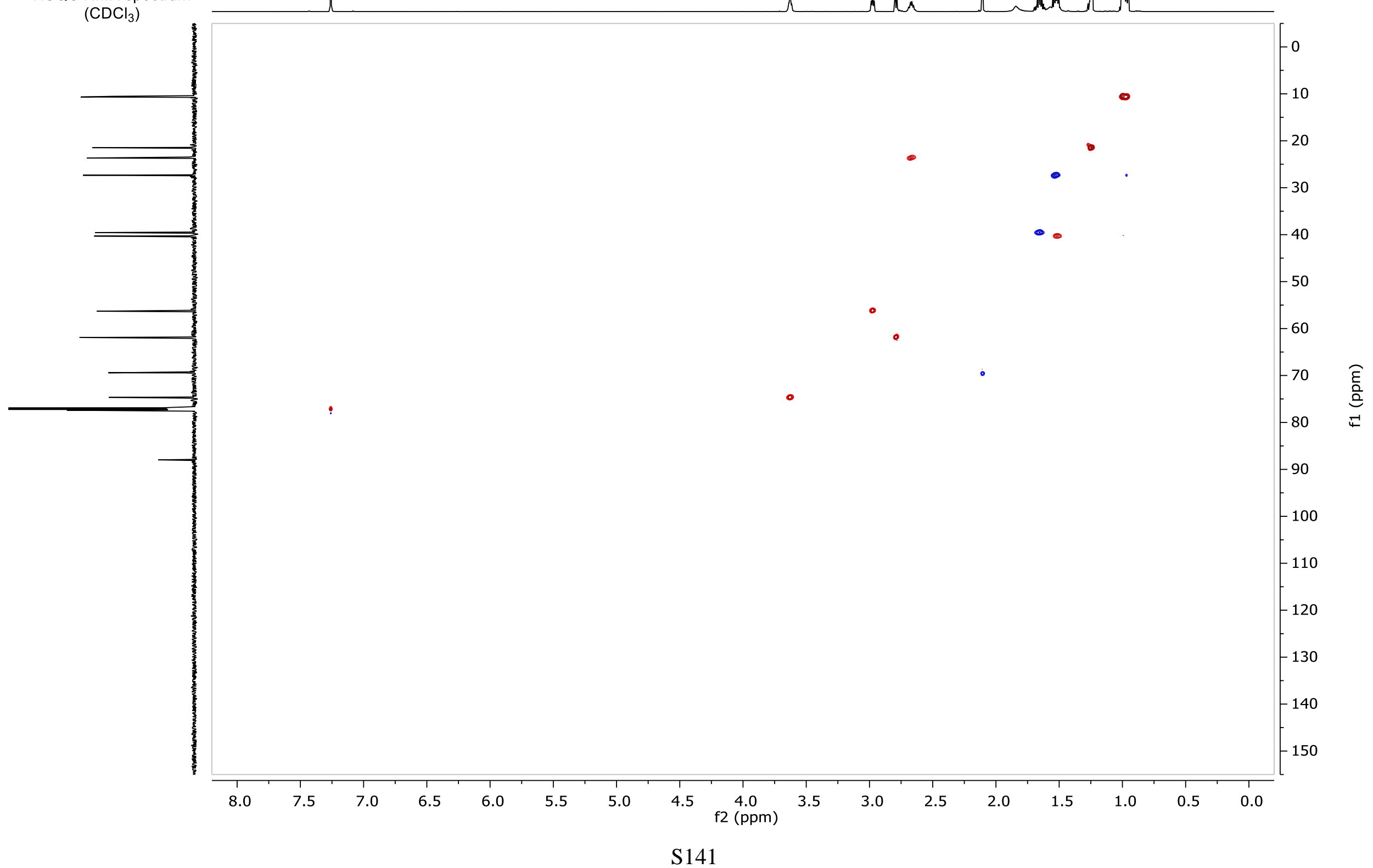


D. Rhoades, * et al.

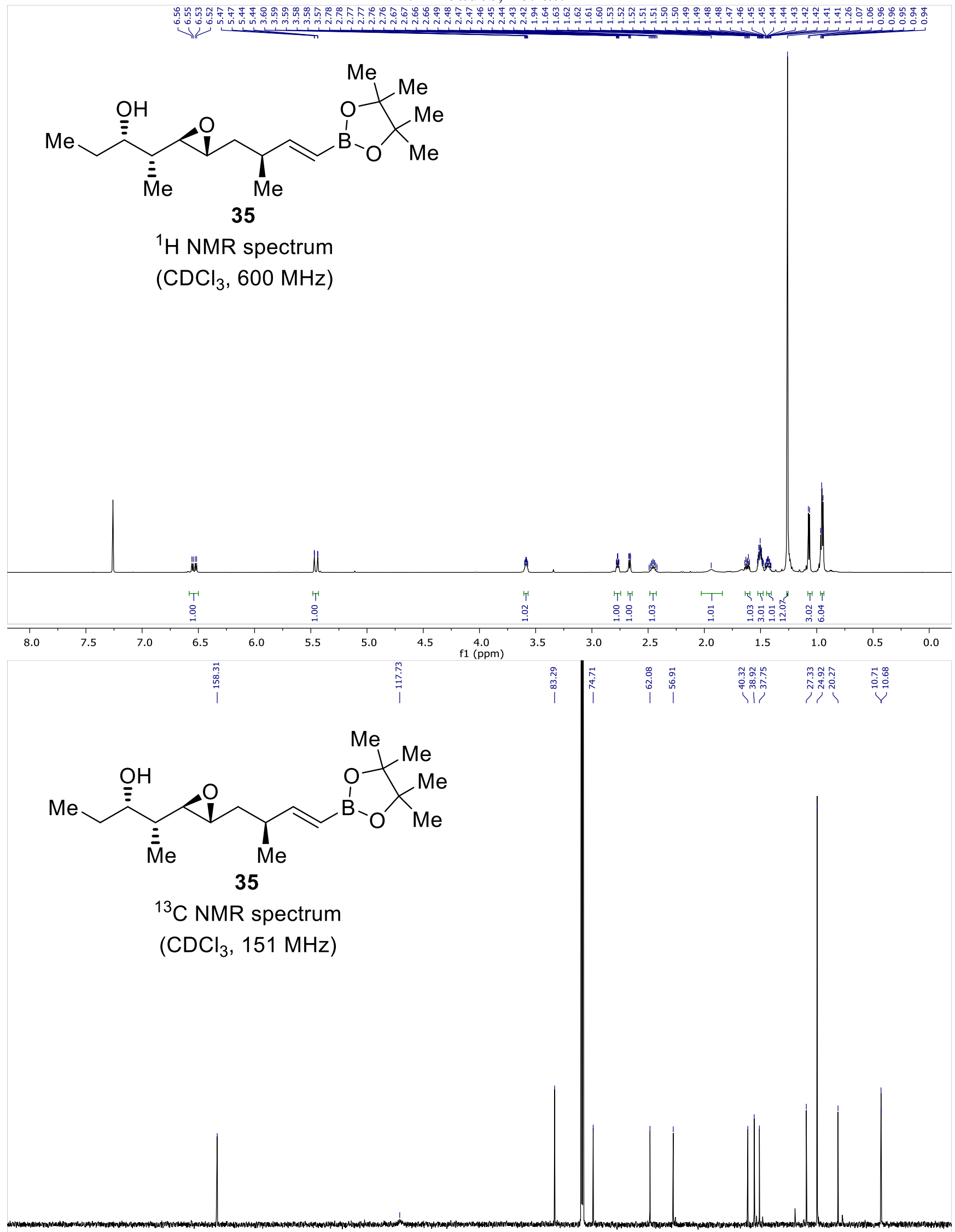


D. Rhoades, * et al.

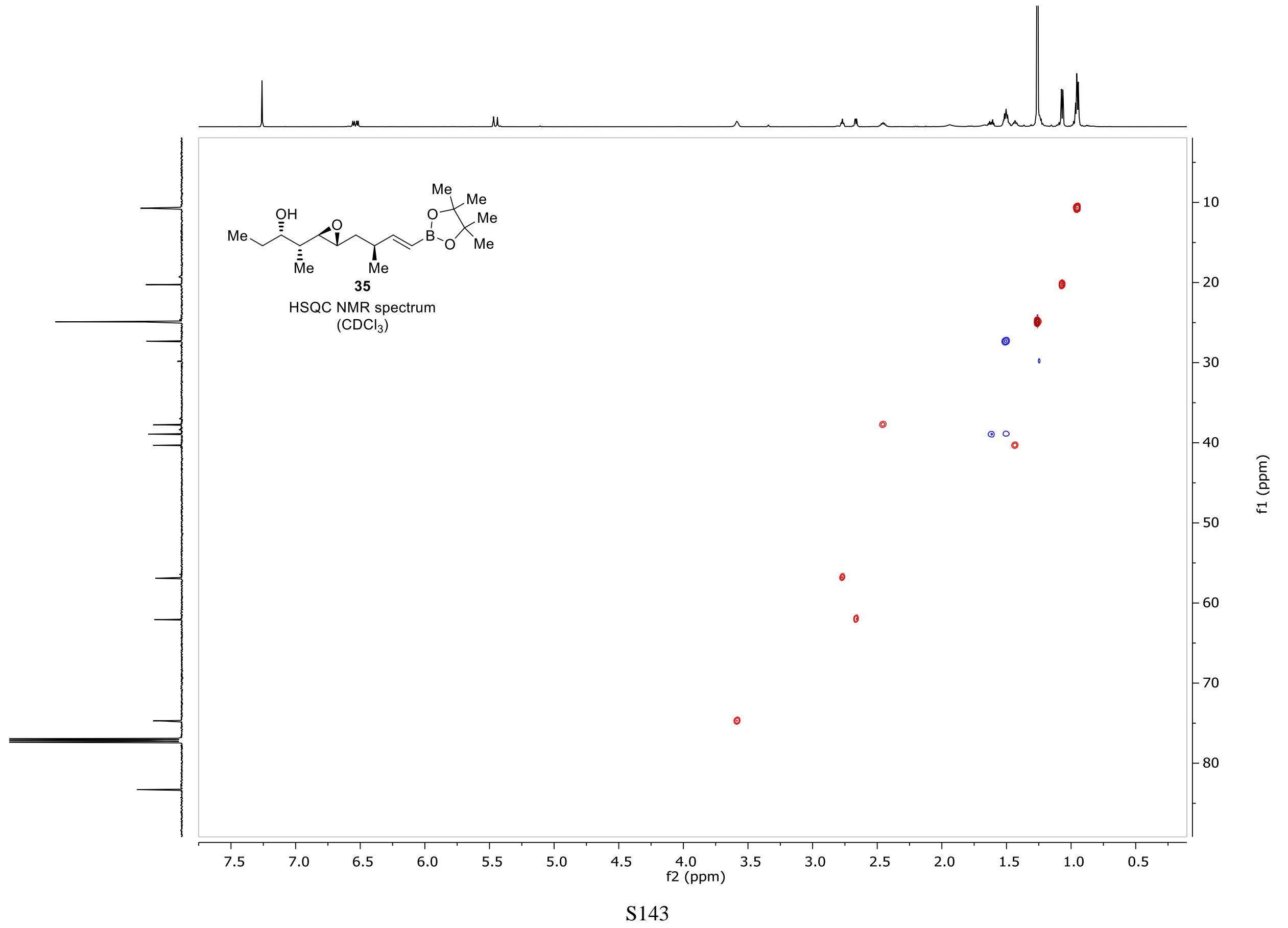


D. Rhoades, * et al.

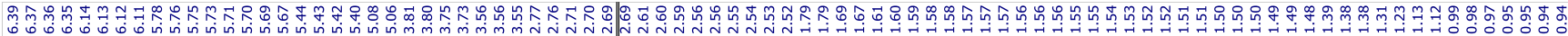

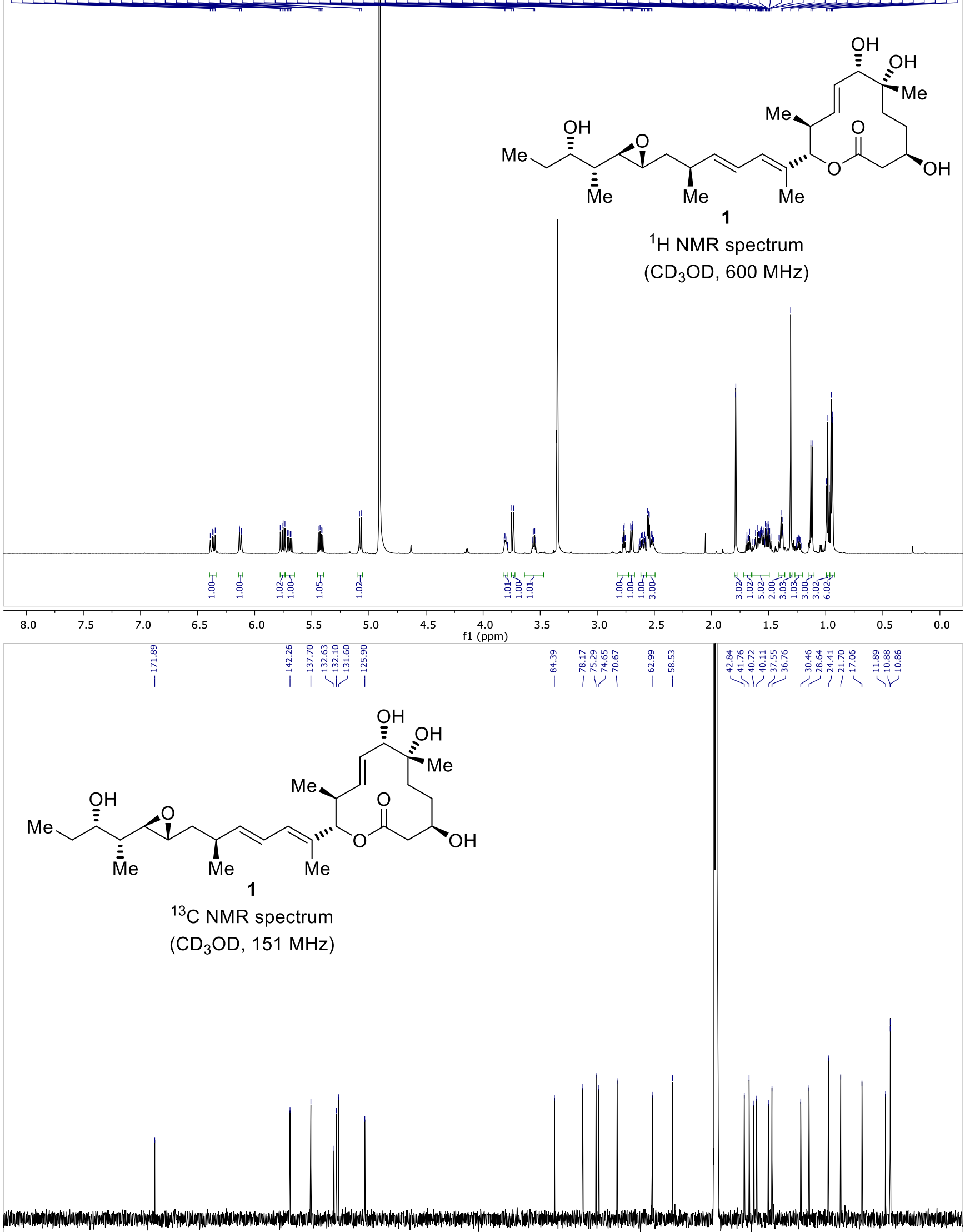


D. Rhoades, * et al.

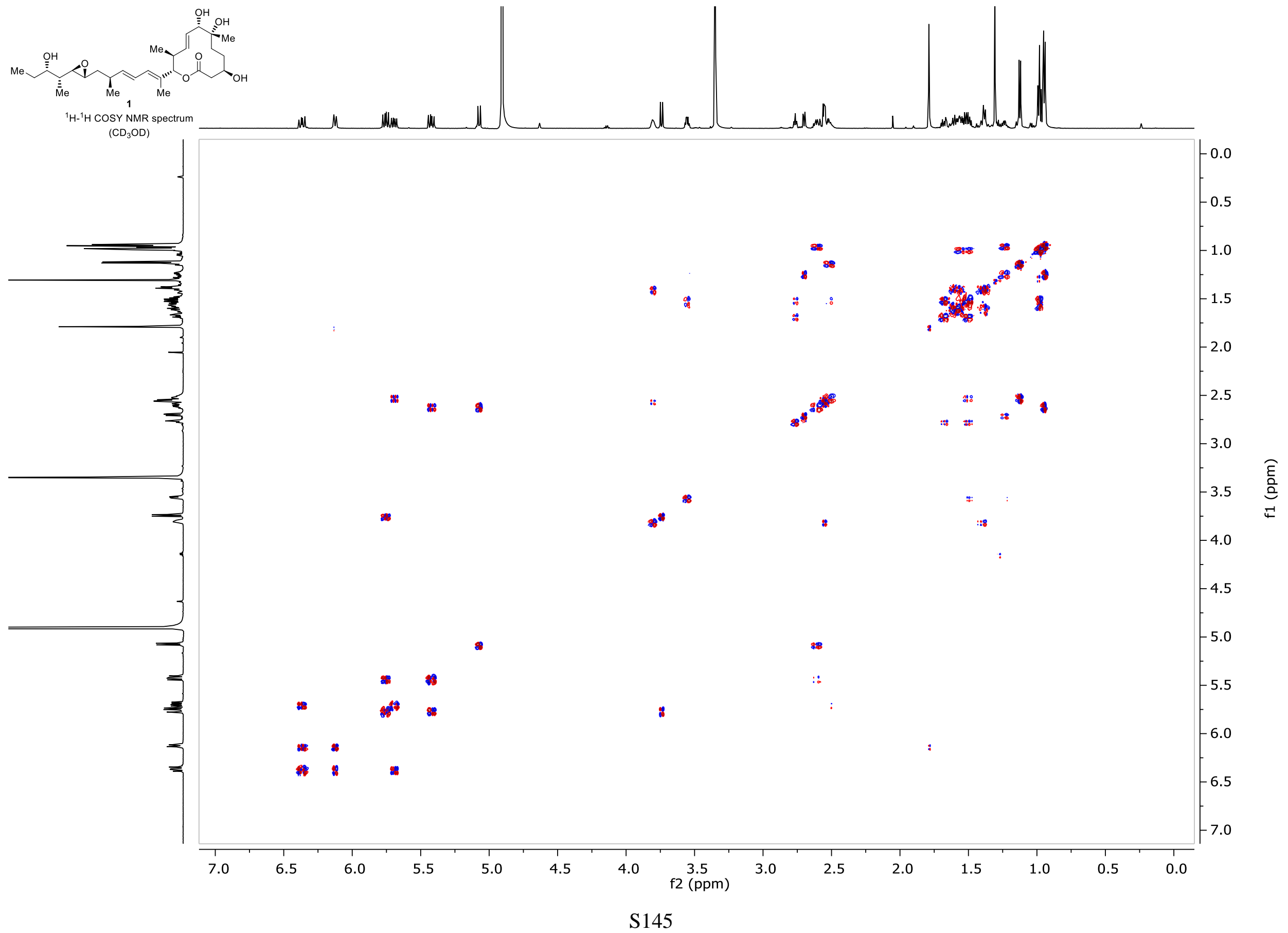


D. Rhoades, ${ }^{*}$ et al.

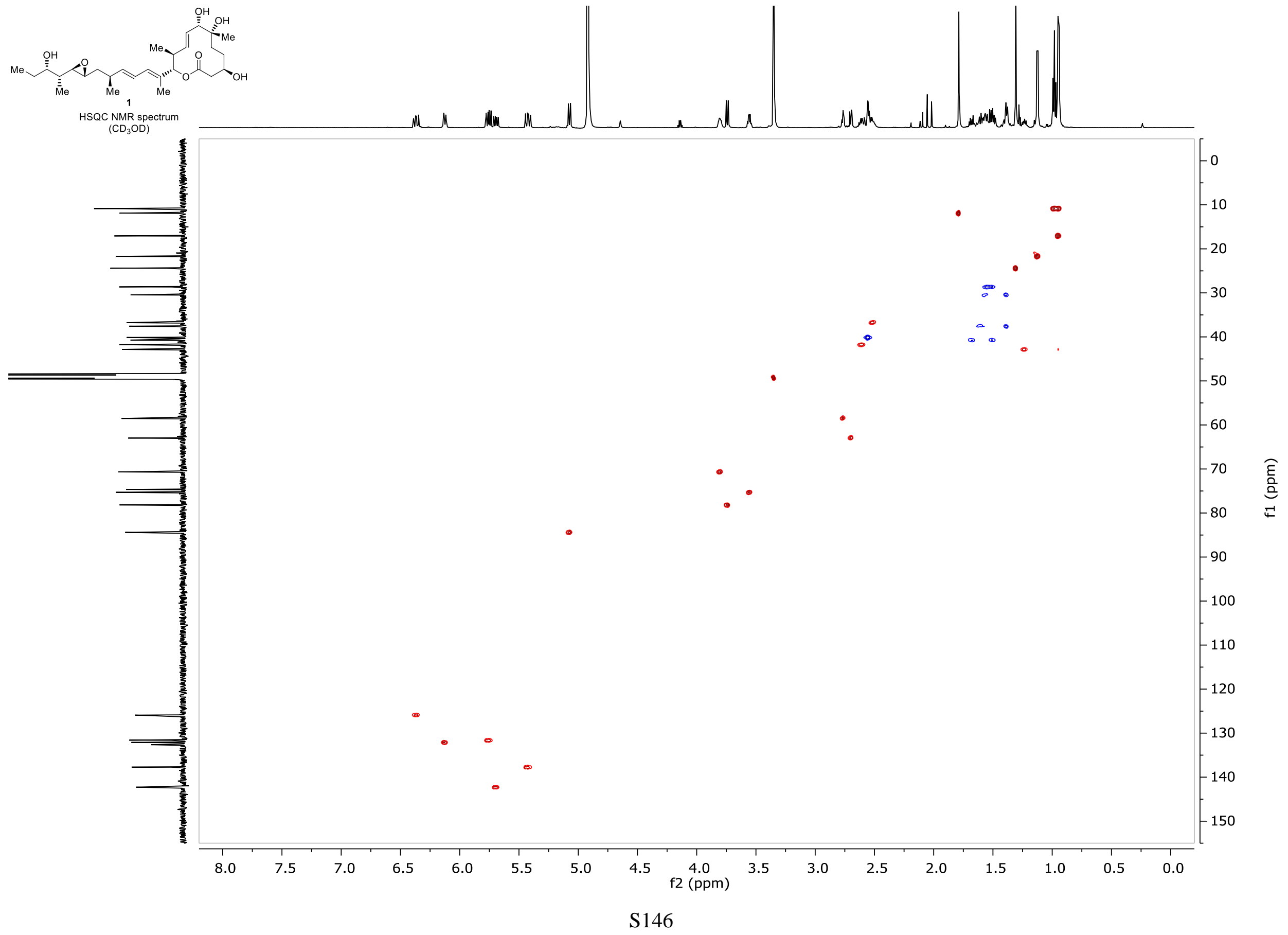


D. Rhoades, ${ }^{*}$ et al.

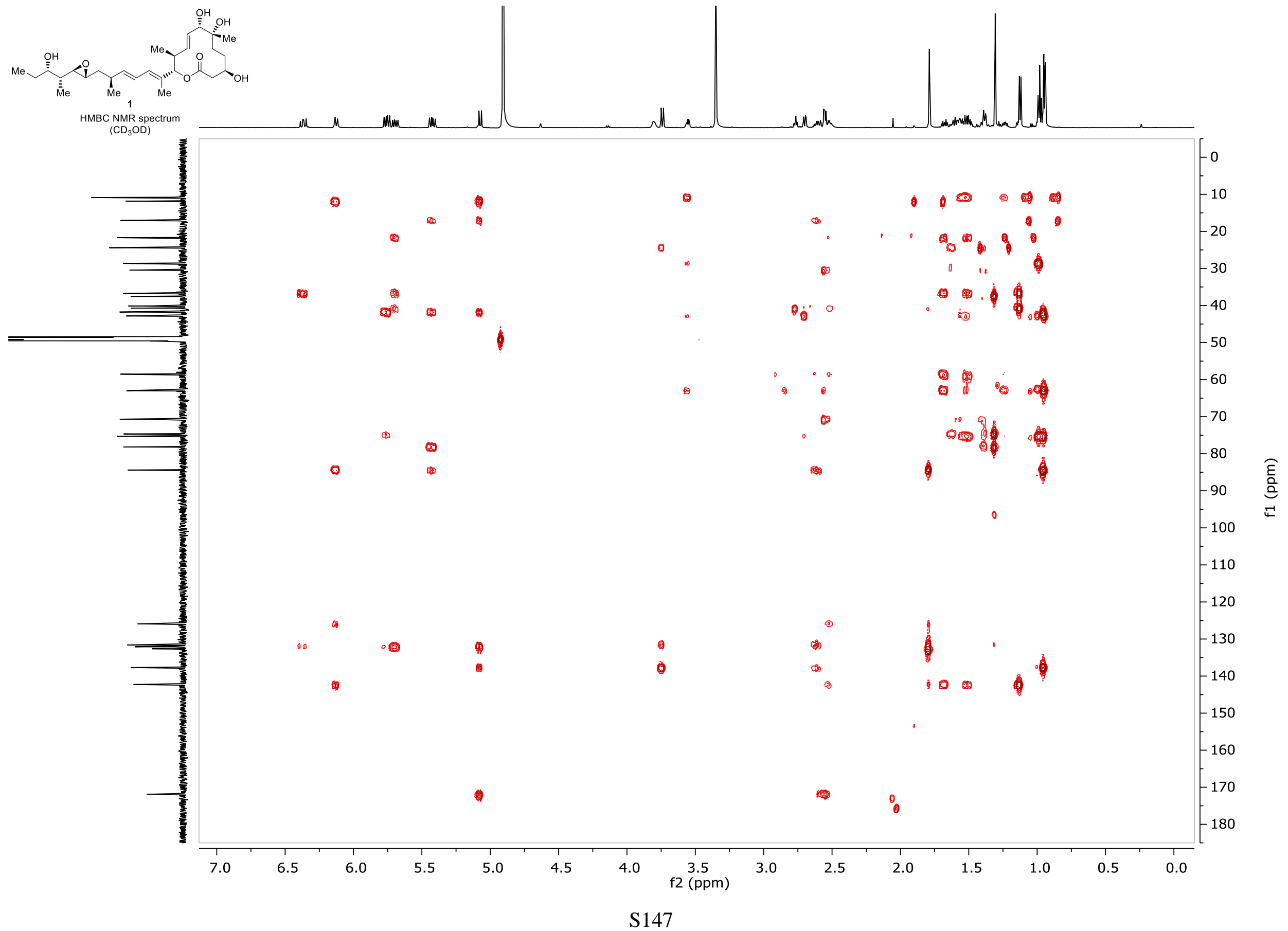


D. Rhoades, * et al.

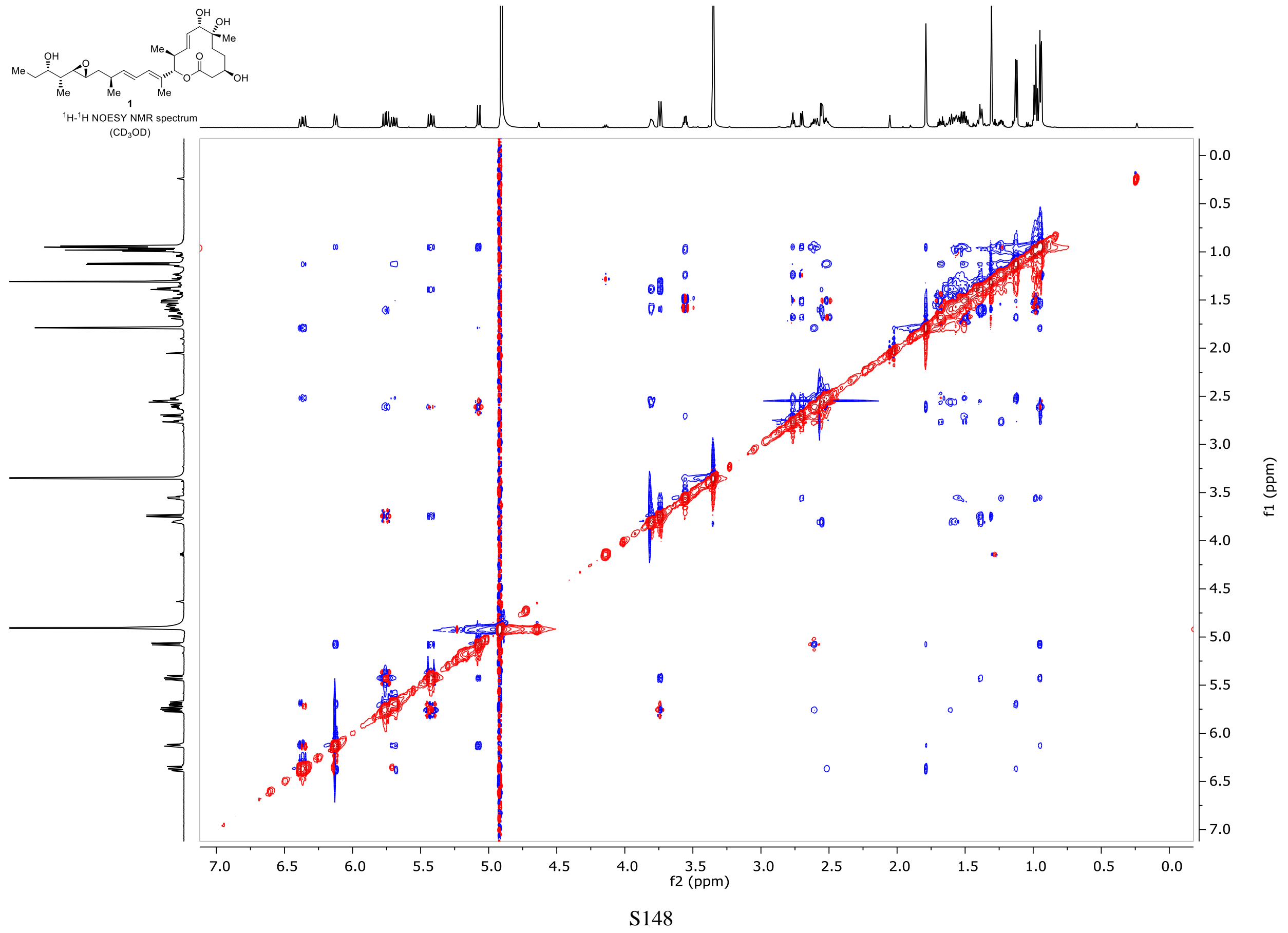


D. Rhoades, * et al.

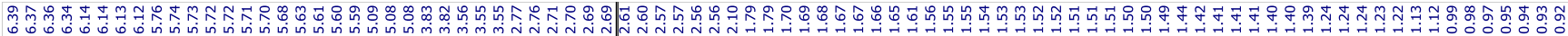

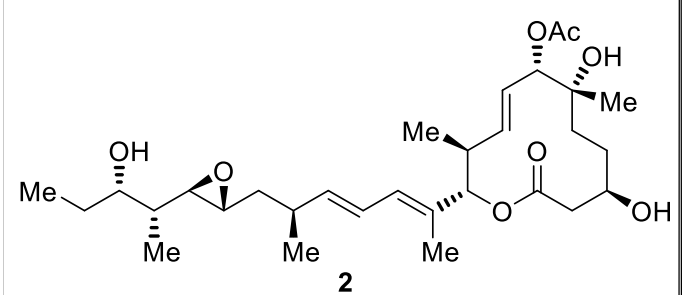

${ }^{1} \mathrm{H}$ NMR spectrum $\left(\mathrm{CD}_{3} \mathrm{OD}, 600 \mathrm{MHz}\right)$

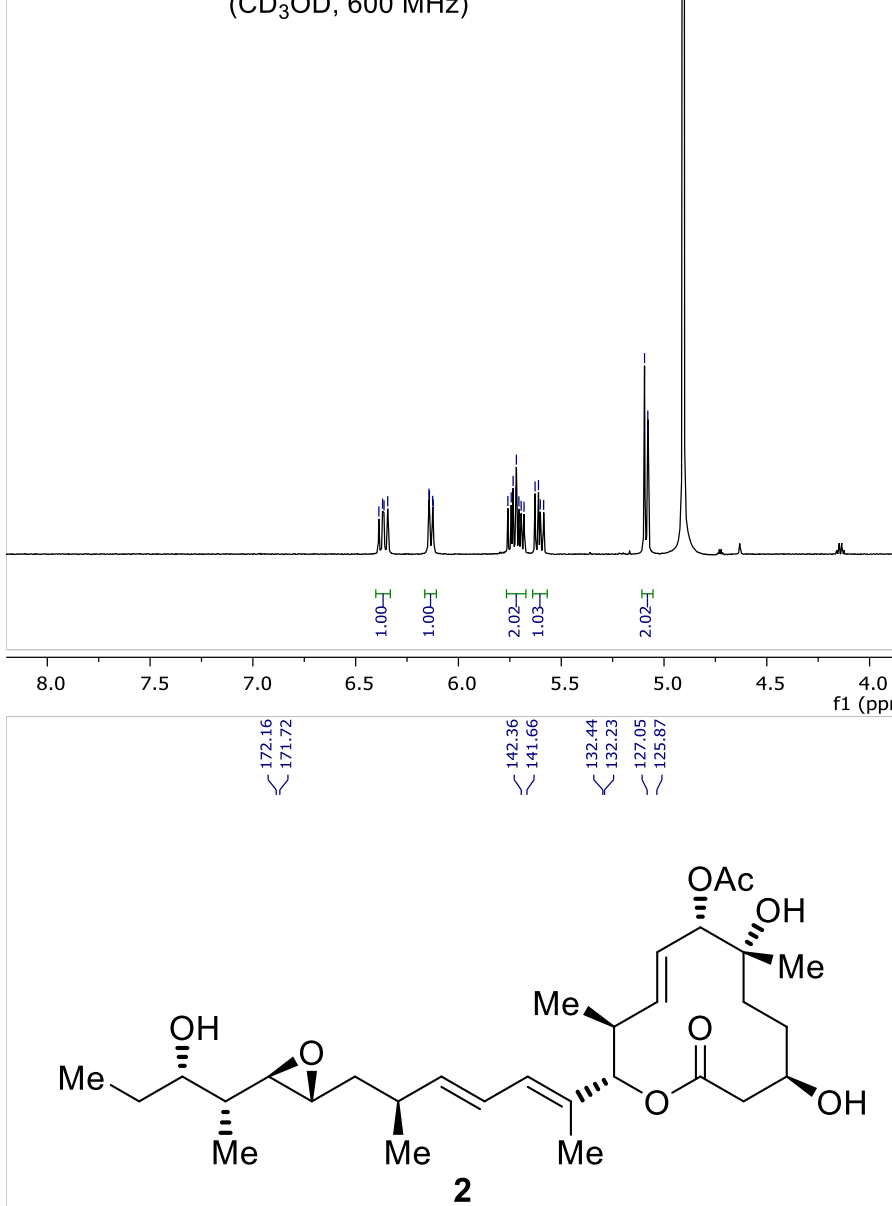

${ }^{13} \mathrm{C}$ NMR spectrum $\left(\mathrm{CD}_{3} \mathrm{OD}, 151 \mathrm{MHz}\right)$ 
D. Rhoades, * et al.

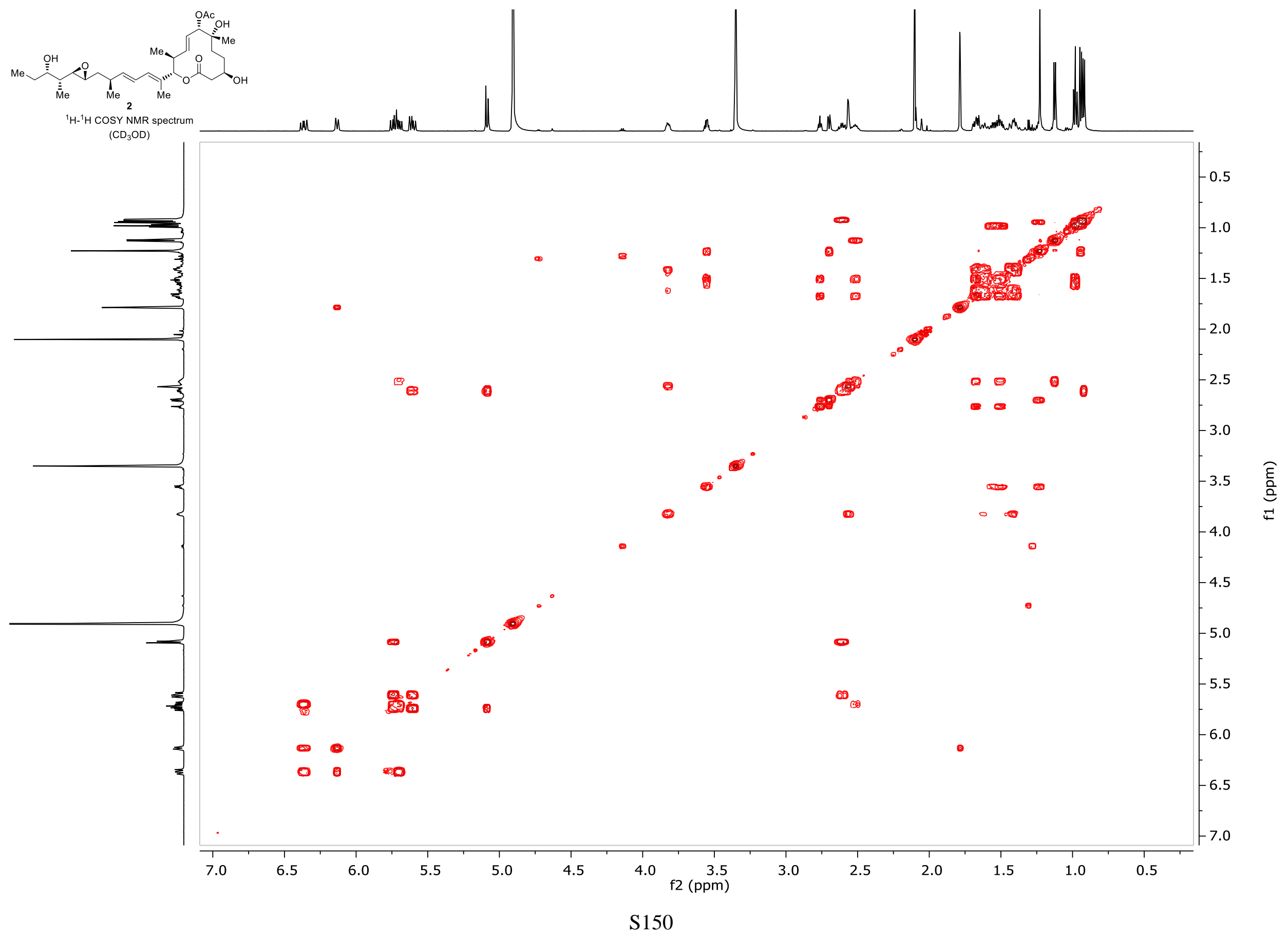


D. Rhoades, ${ }^{*}$ et al.

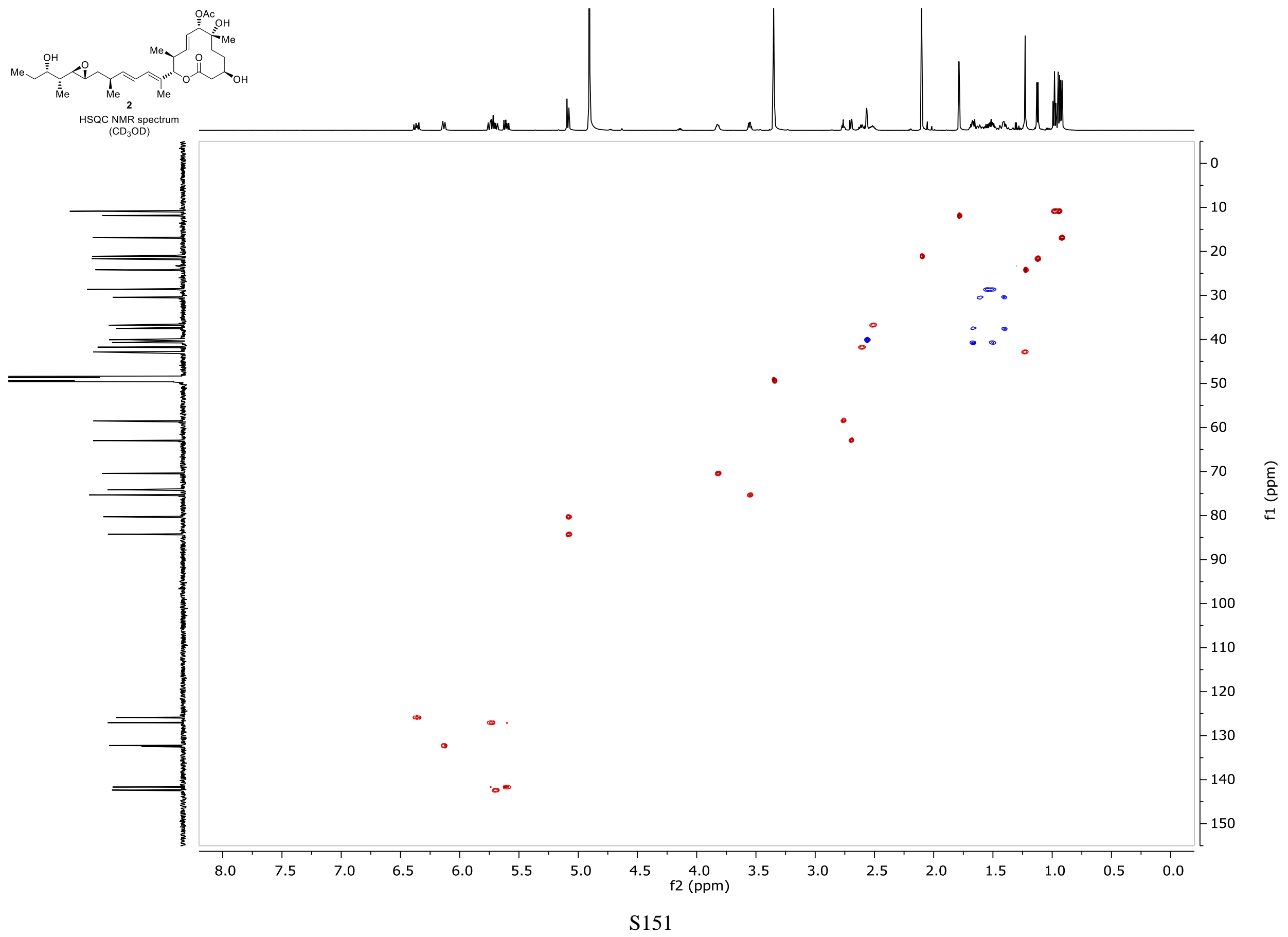


D. Rhoades, * et al.

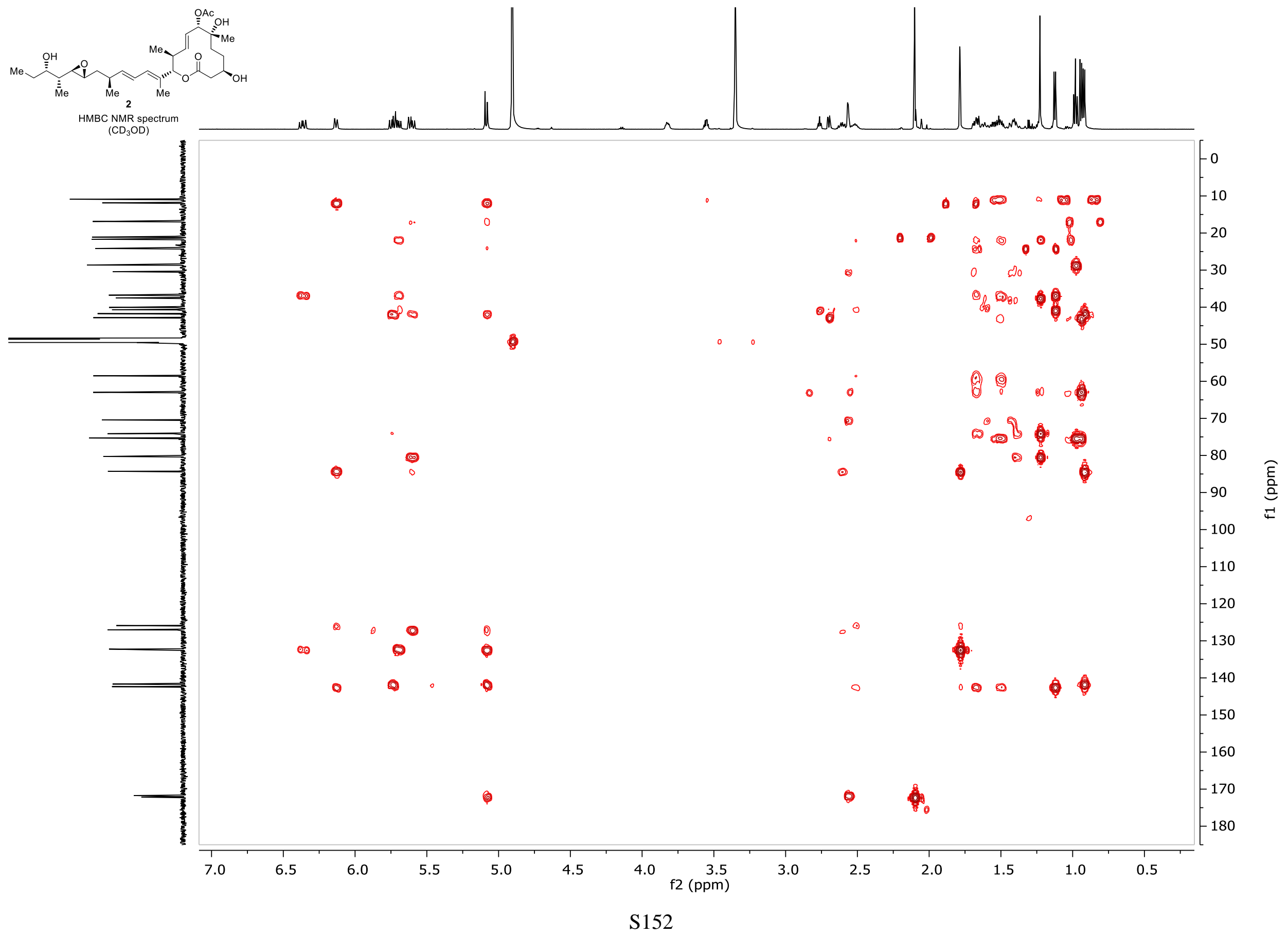


D. Rhoades, * et al.

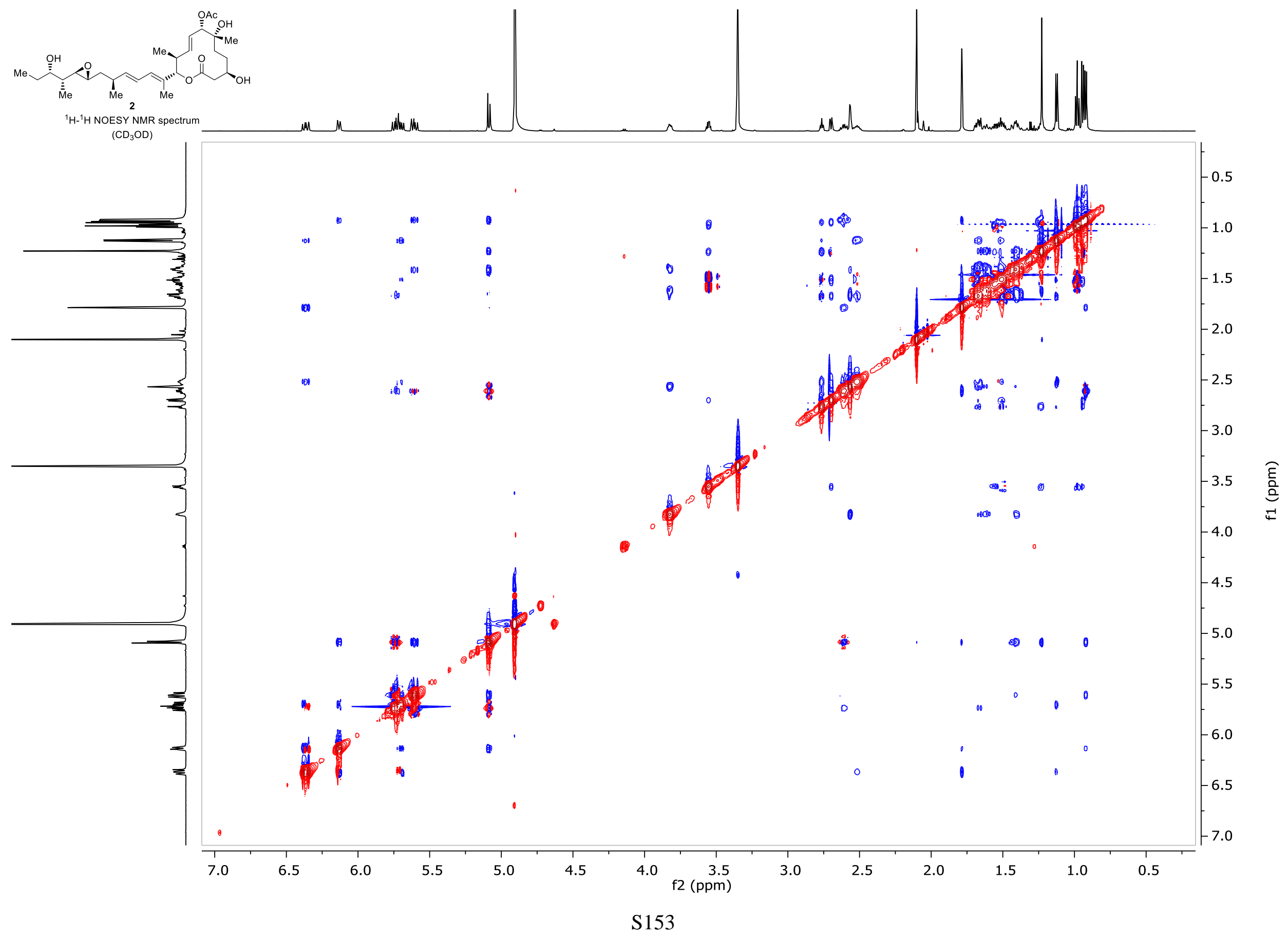


D. Rhoades, * et al.<smiles>CC(=CC#N)c1ccccn1</smiles>

10

${ }^{1} \mathrm{H}$ NMR spectrum

$\left(\mathrm{CDCl}_{3}, 600 \mathrm{MHz}\right)$
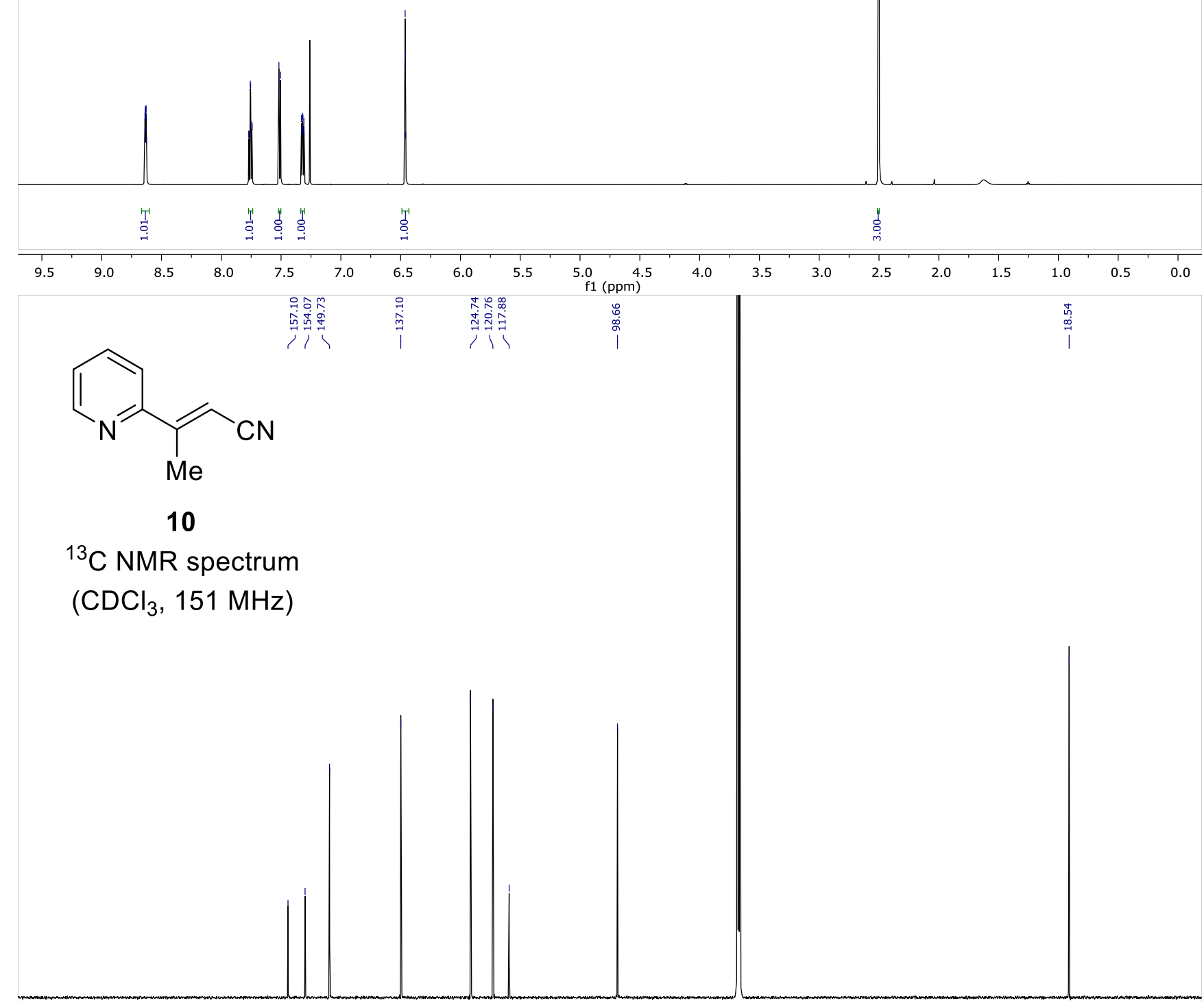
D. Rhoades, * et al.

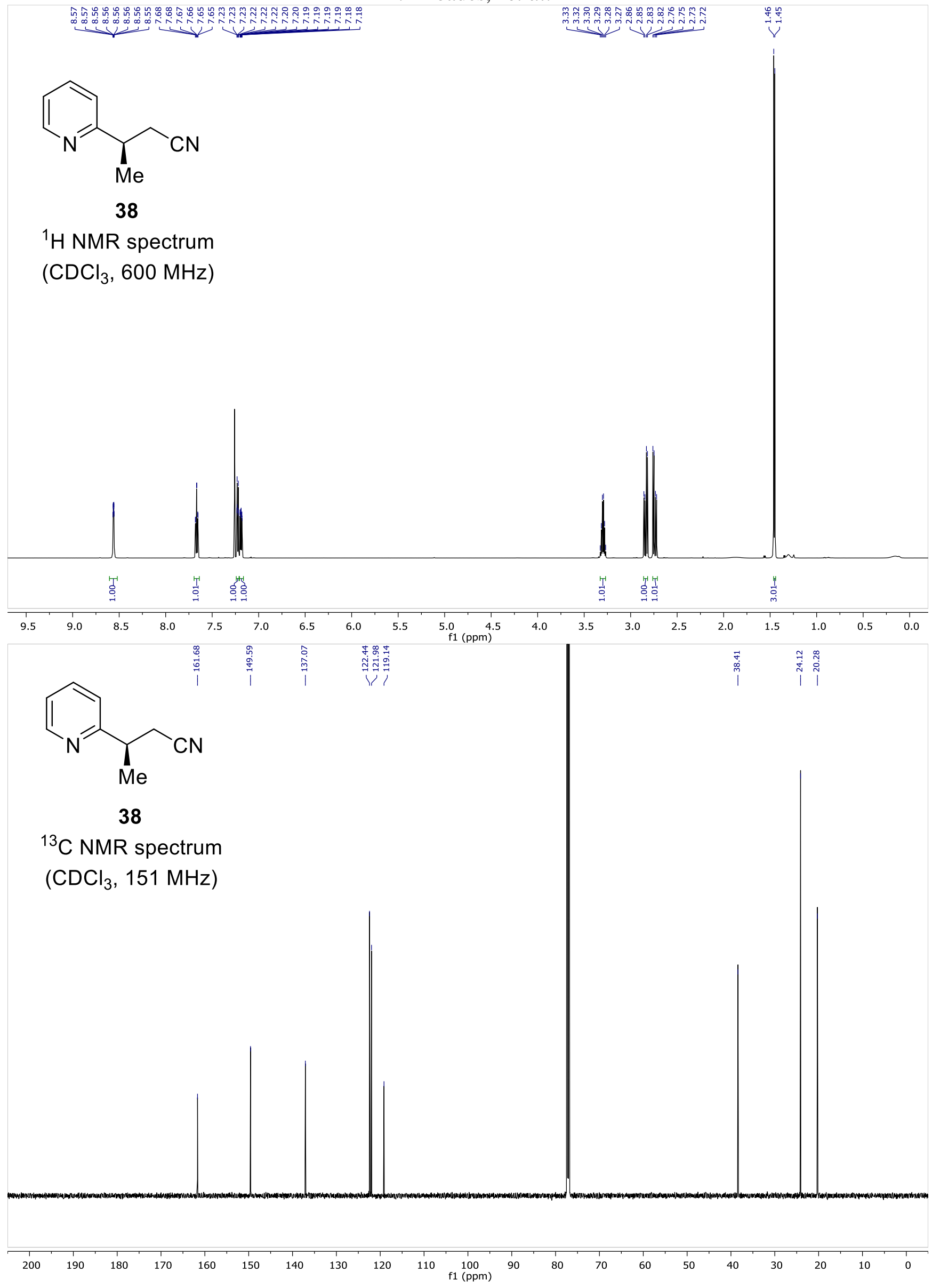


D. Rhoades, * et al.
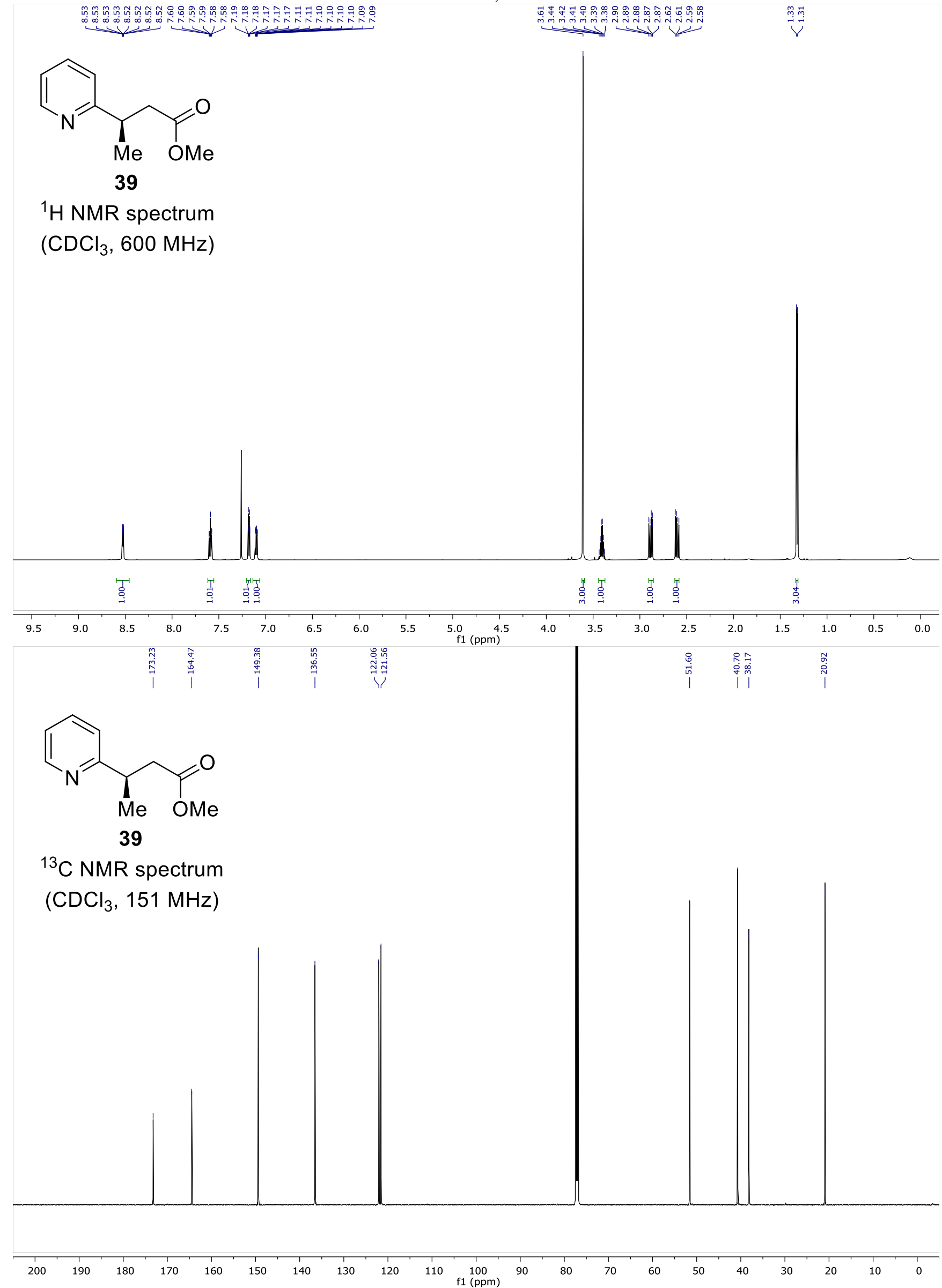


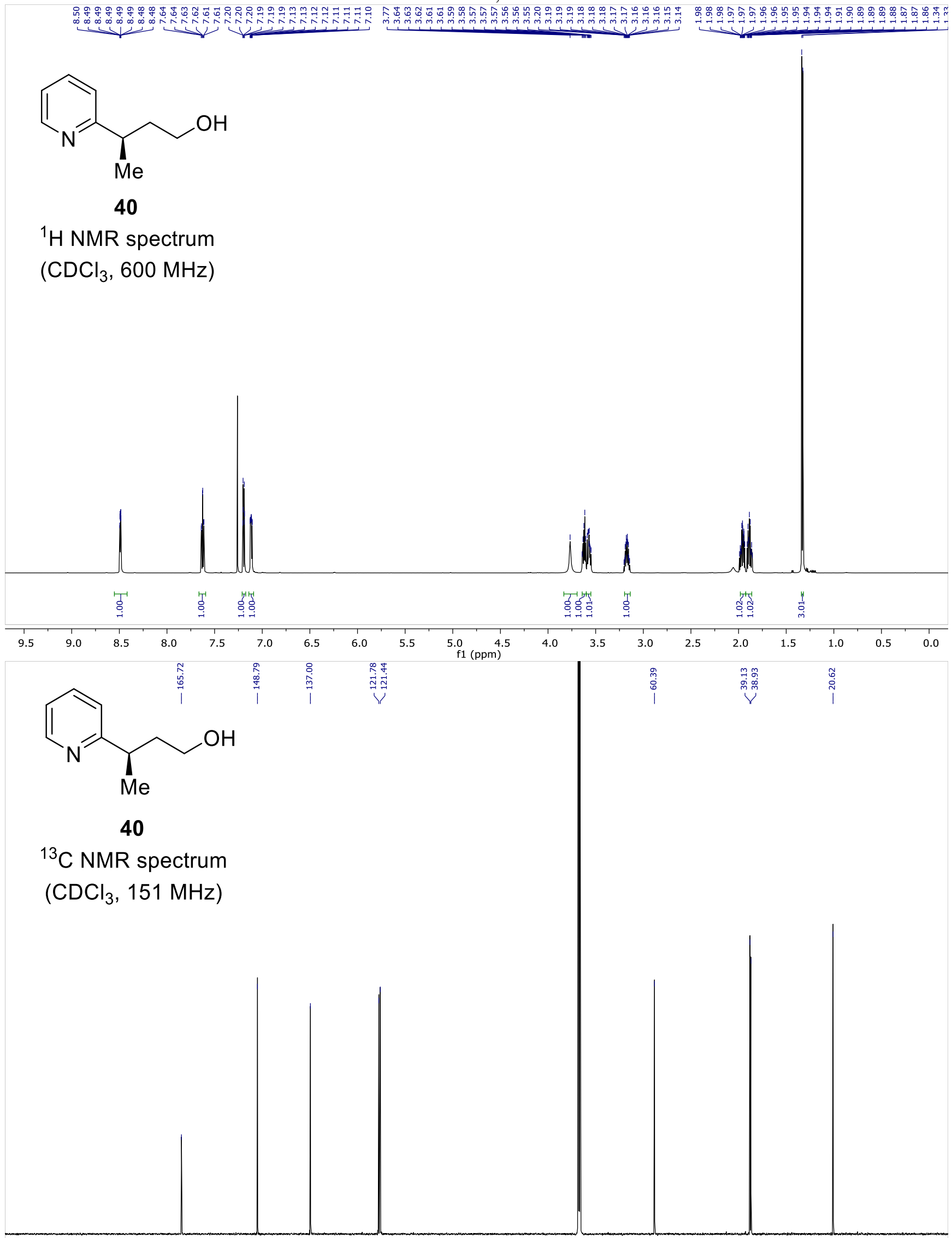


D. Rhoades, * et al.

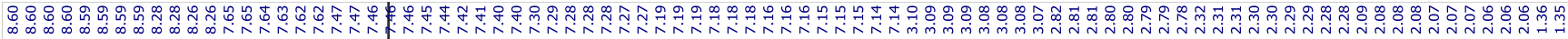

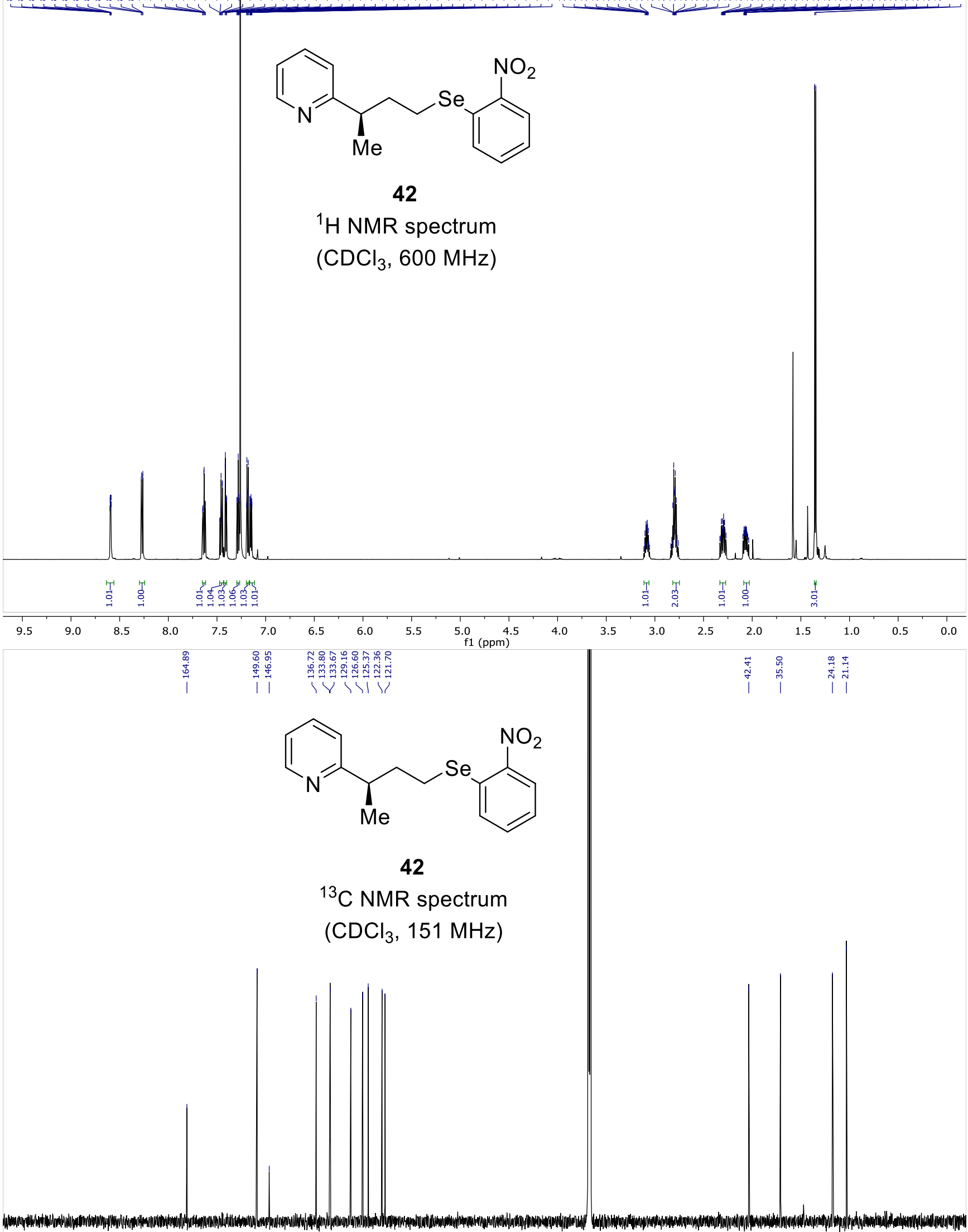


D. Rhoades, * et al.

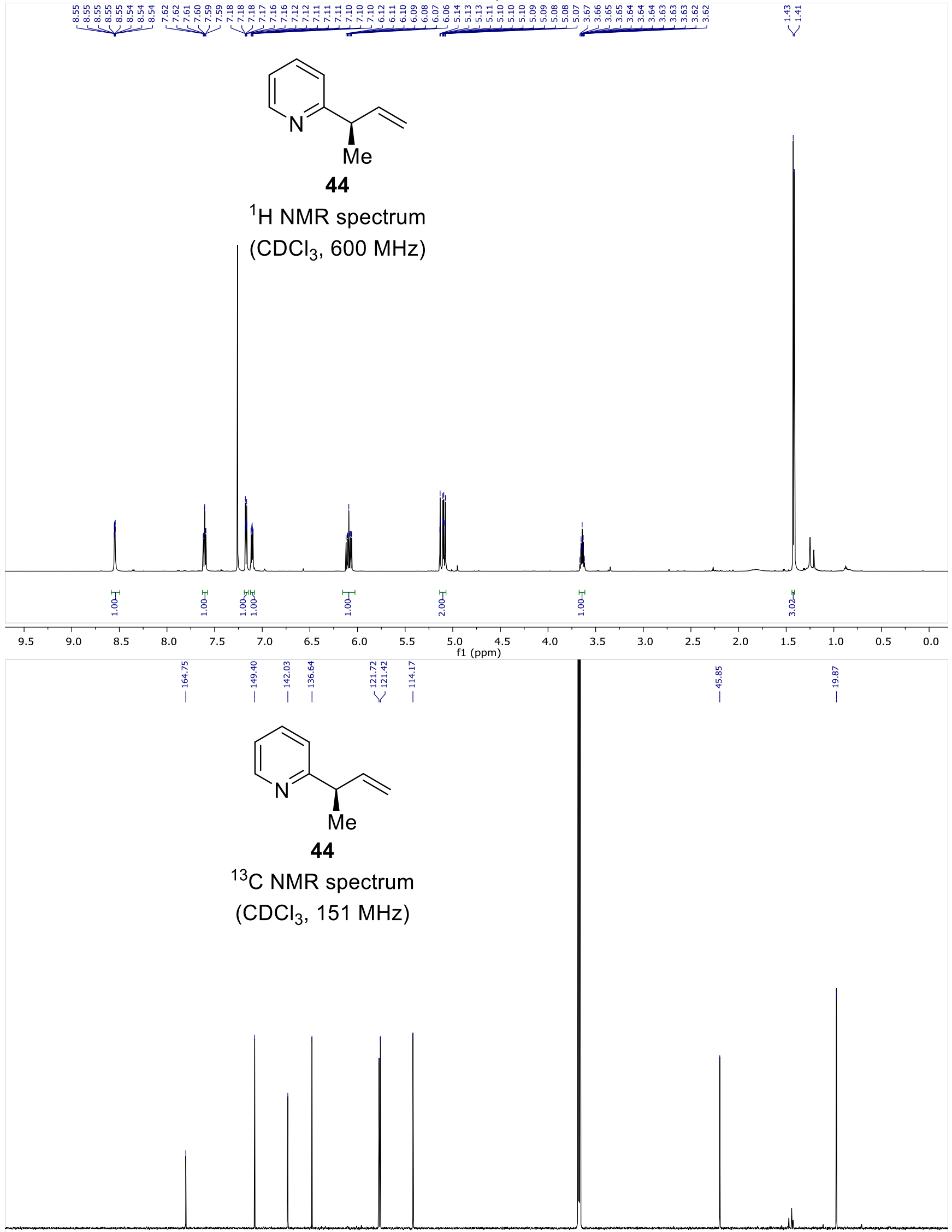


D. Rhoades, * et al.

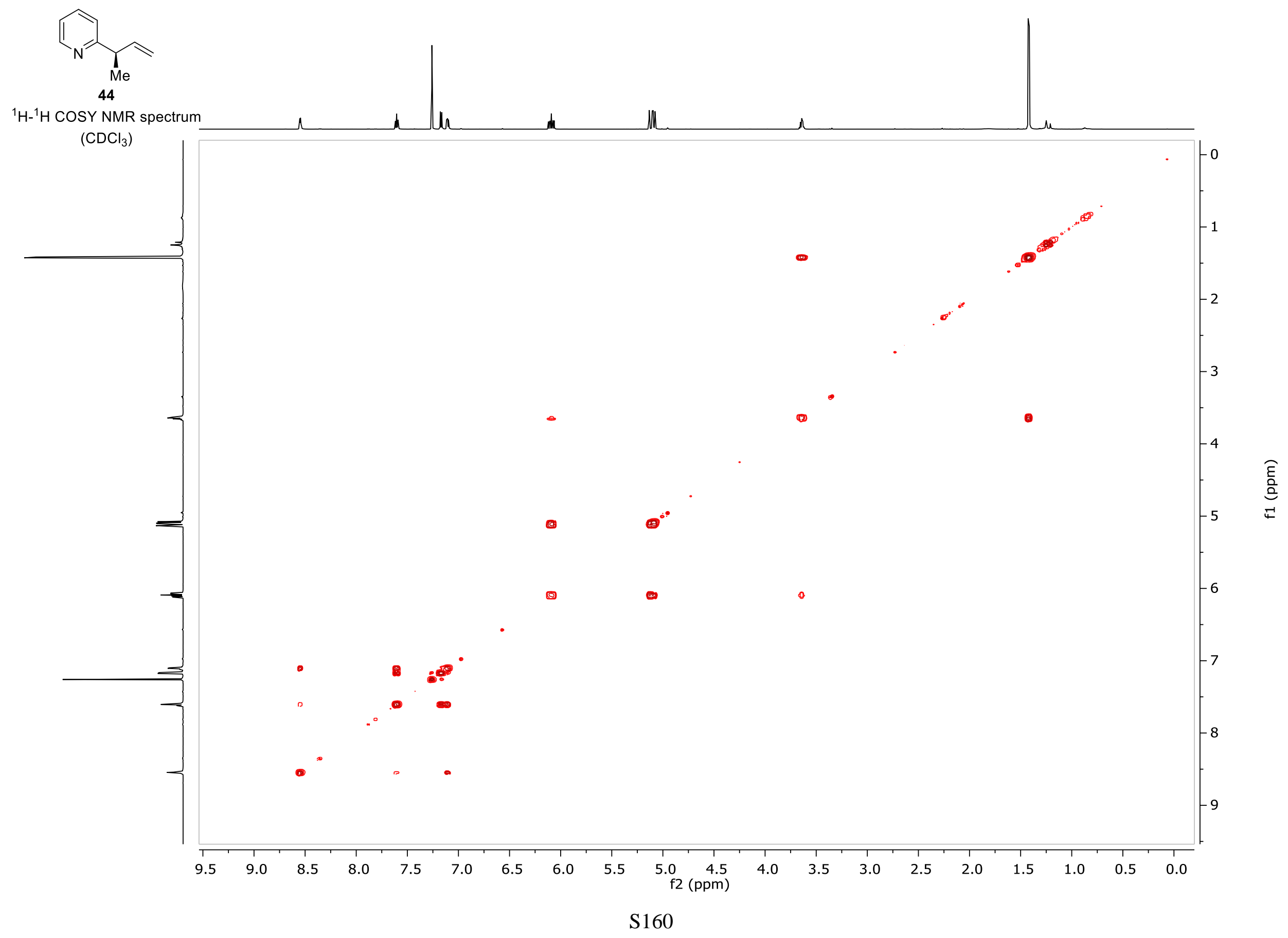


D. Rhoades, * et al.

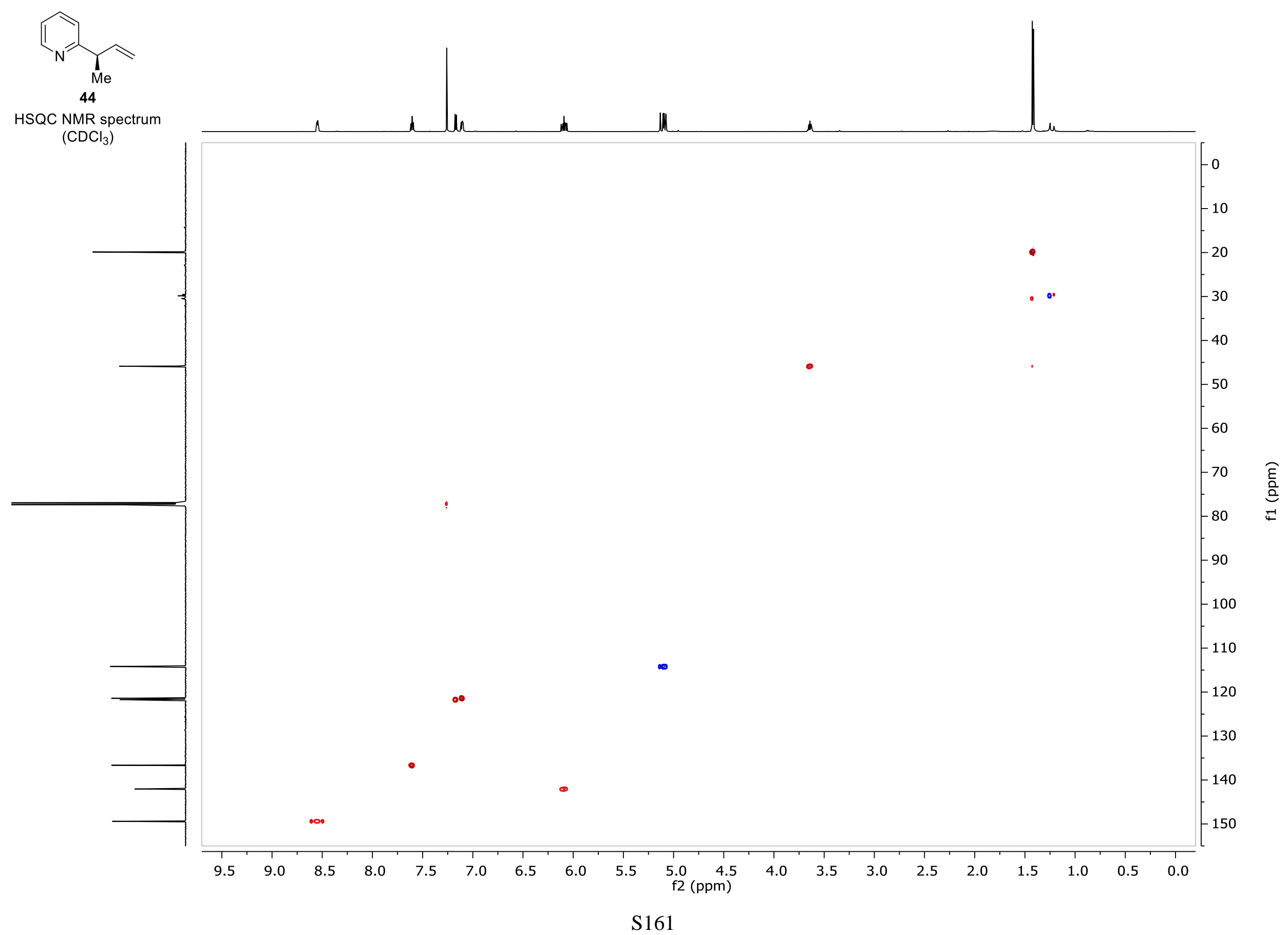




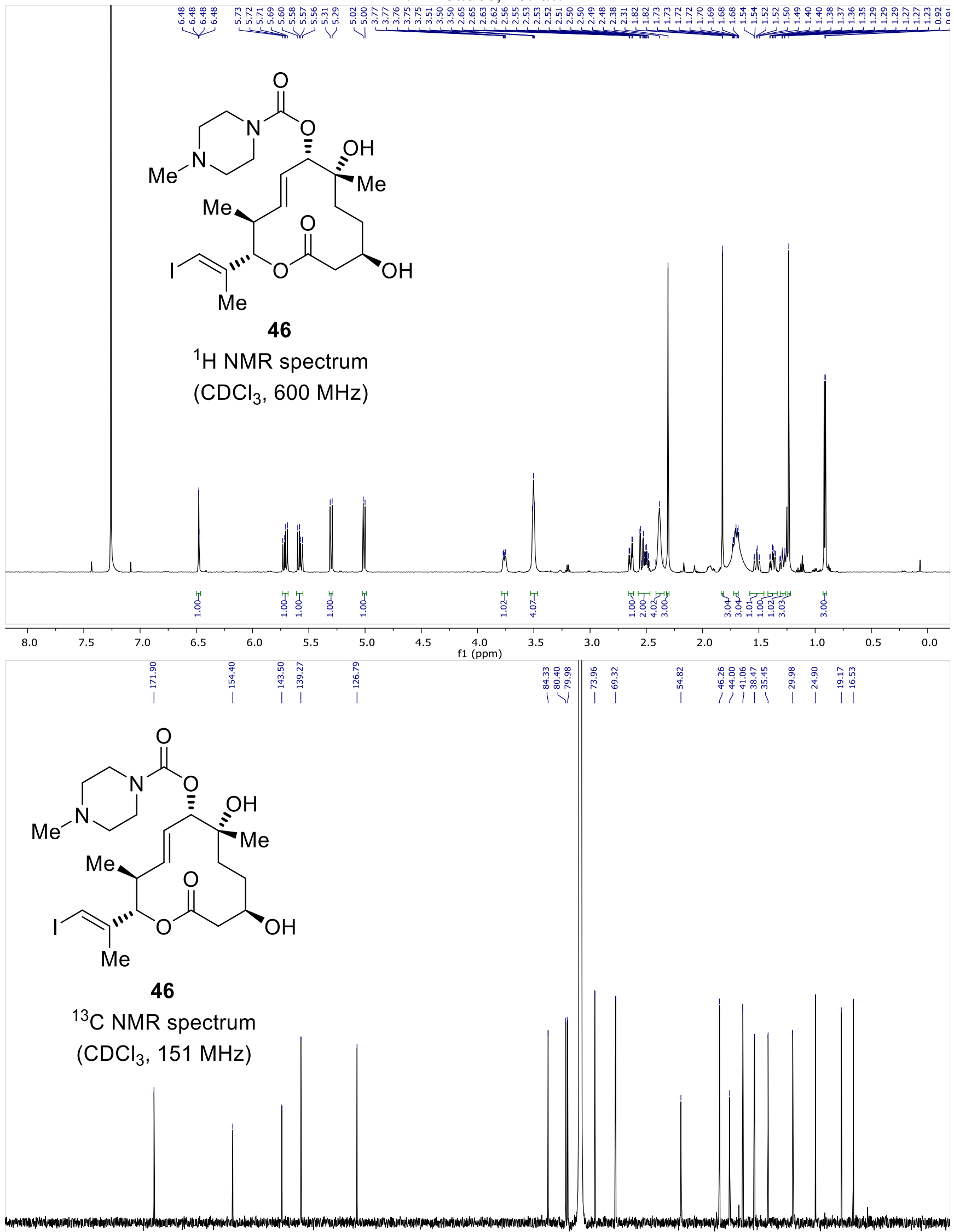


D. Rhoades, * et al.

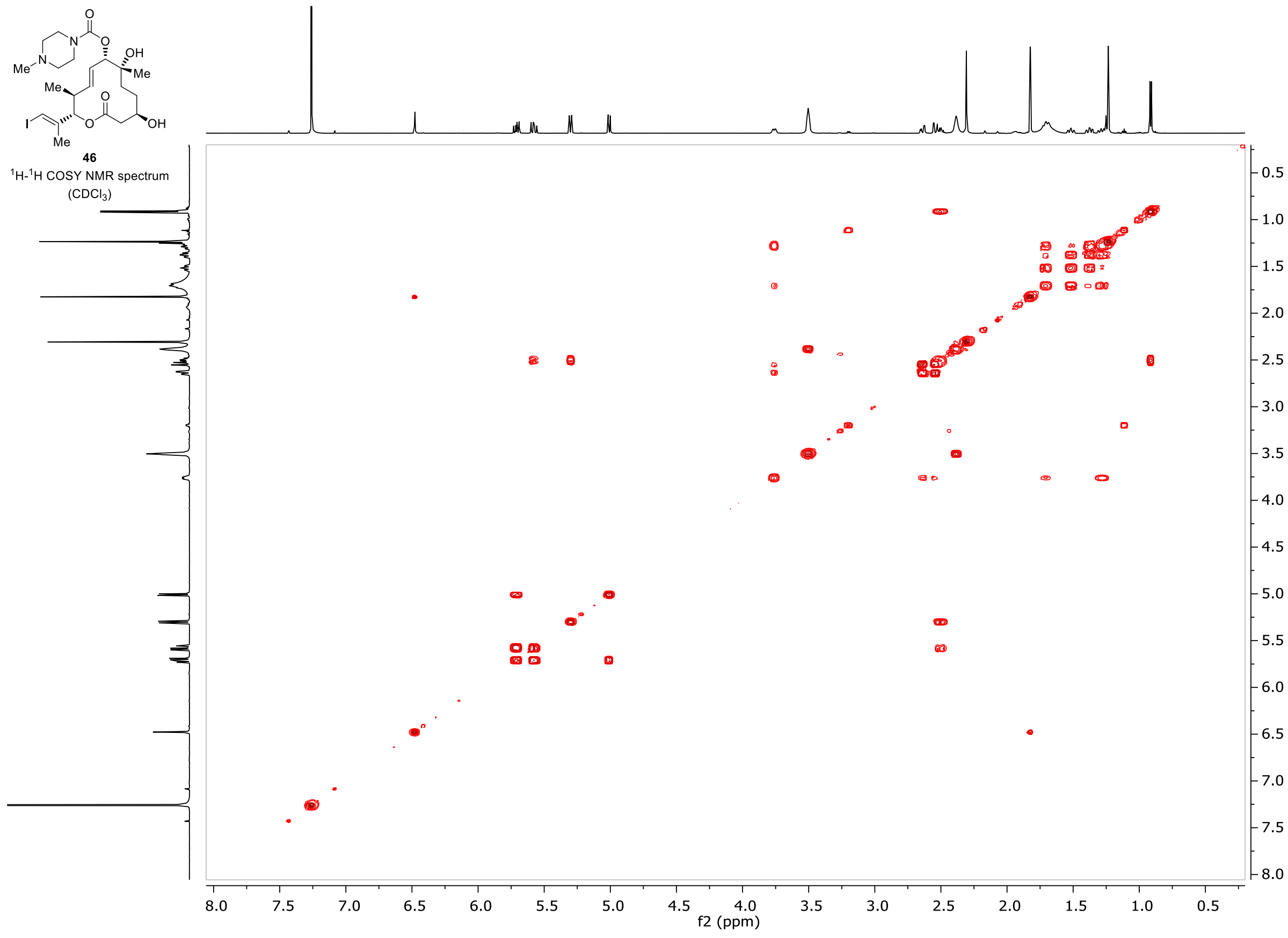

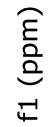

S163 
D. Rhoades,* et al.

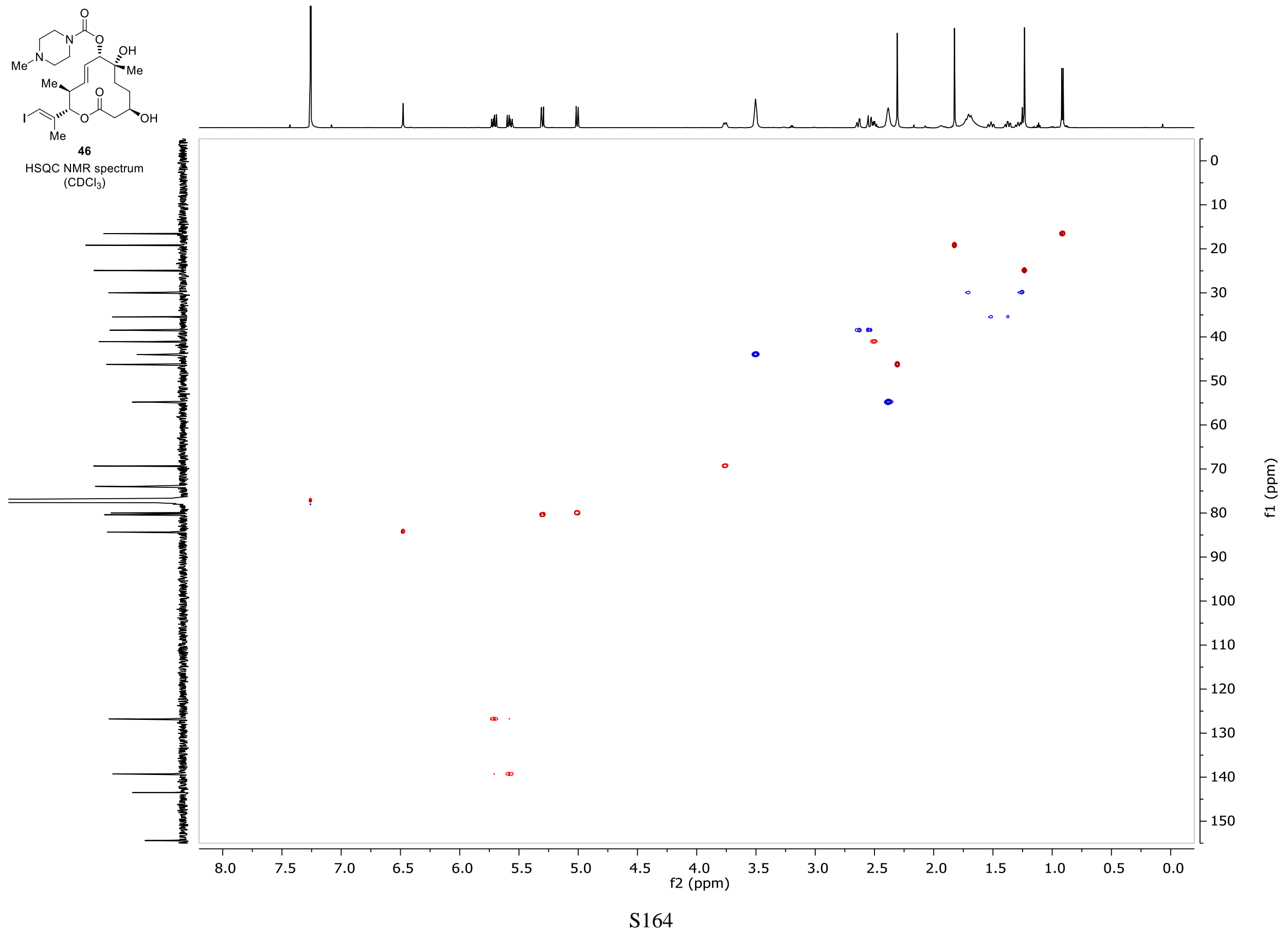


D. Rhoades, * et al.

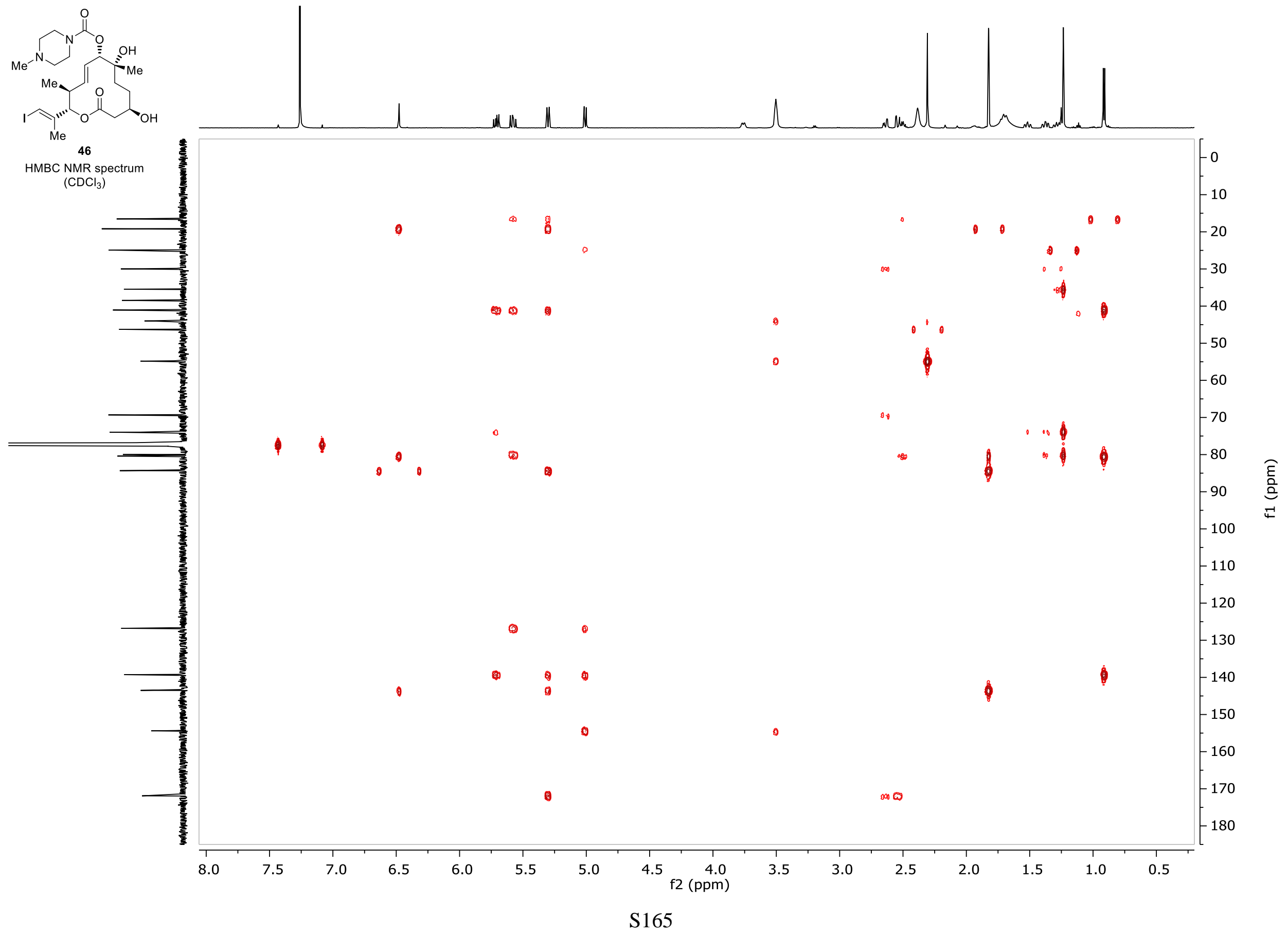


D. Rhoades, ${ }^{*}$ et al.

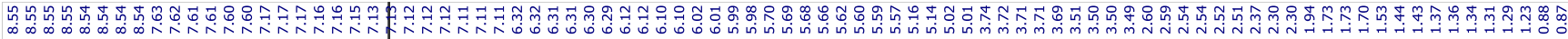

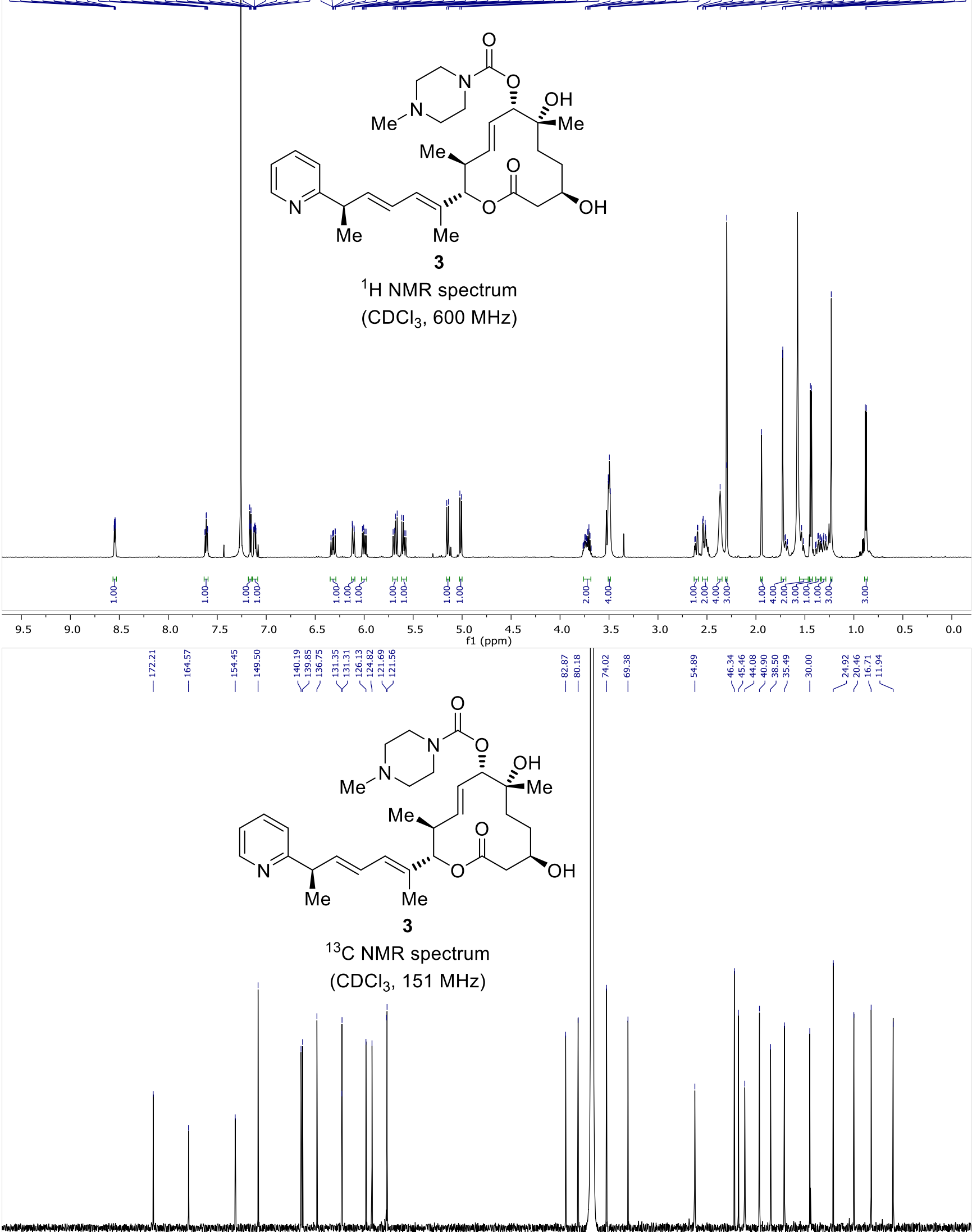


D. Rhoades, * et al.

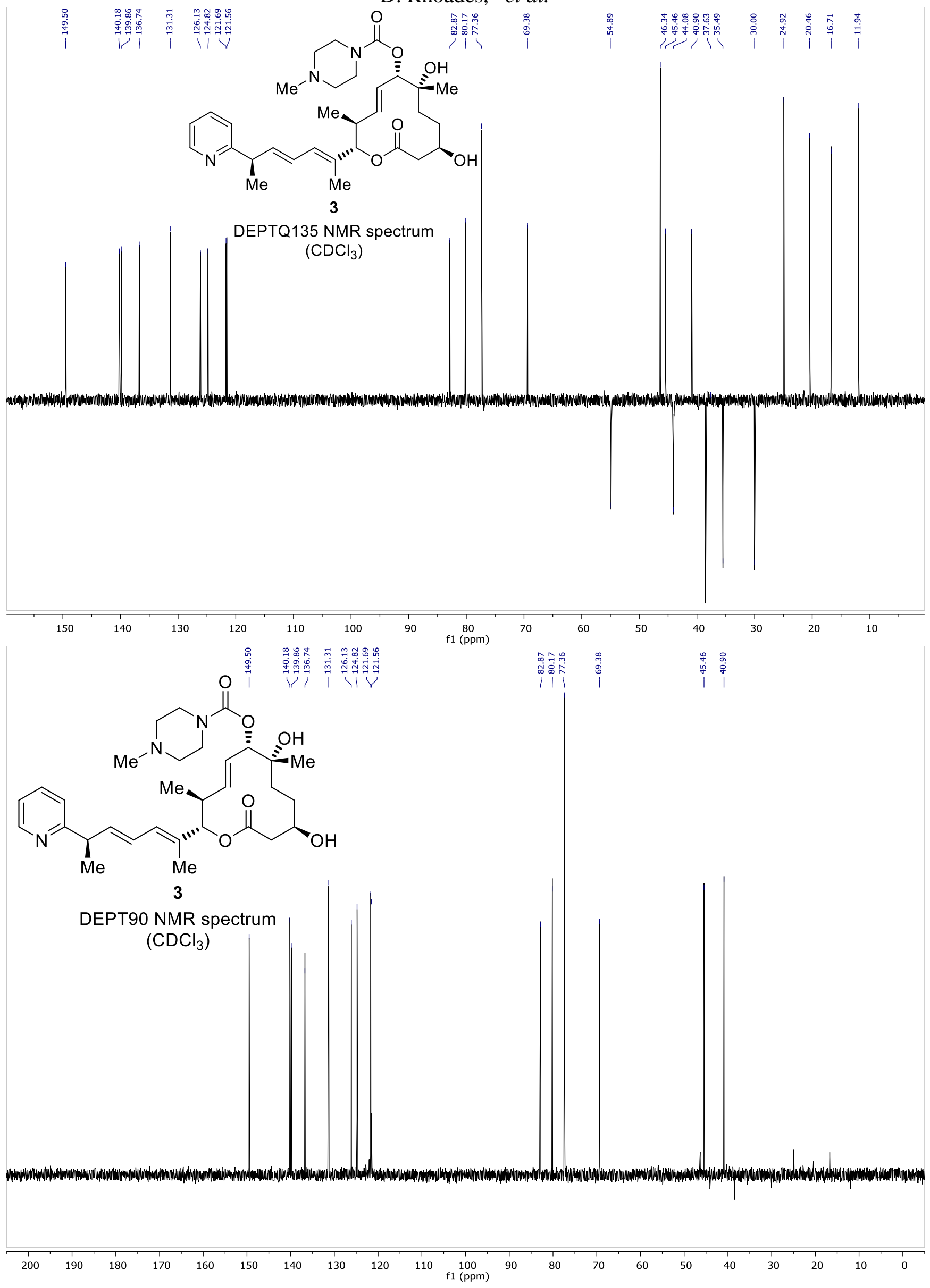


D. Rhoades, * et al.

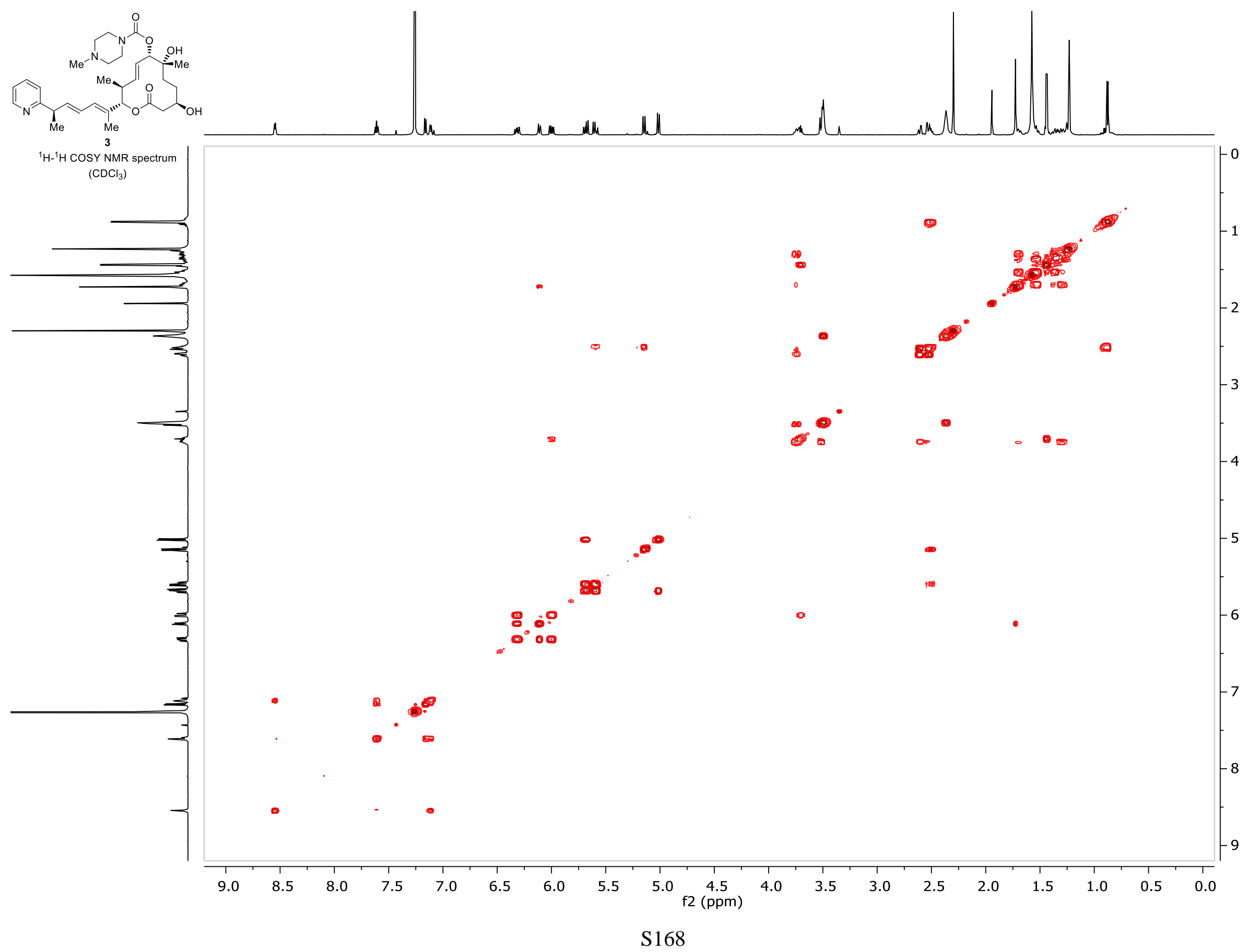

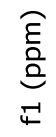


D. Rhoades, ${ }^{*}$ et al.

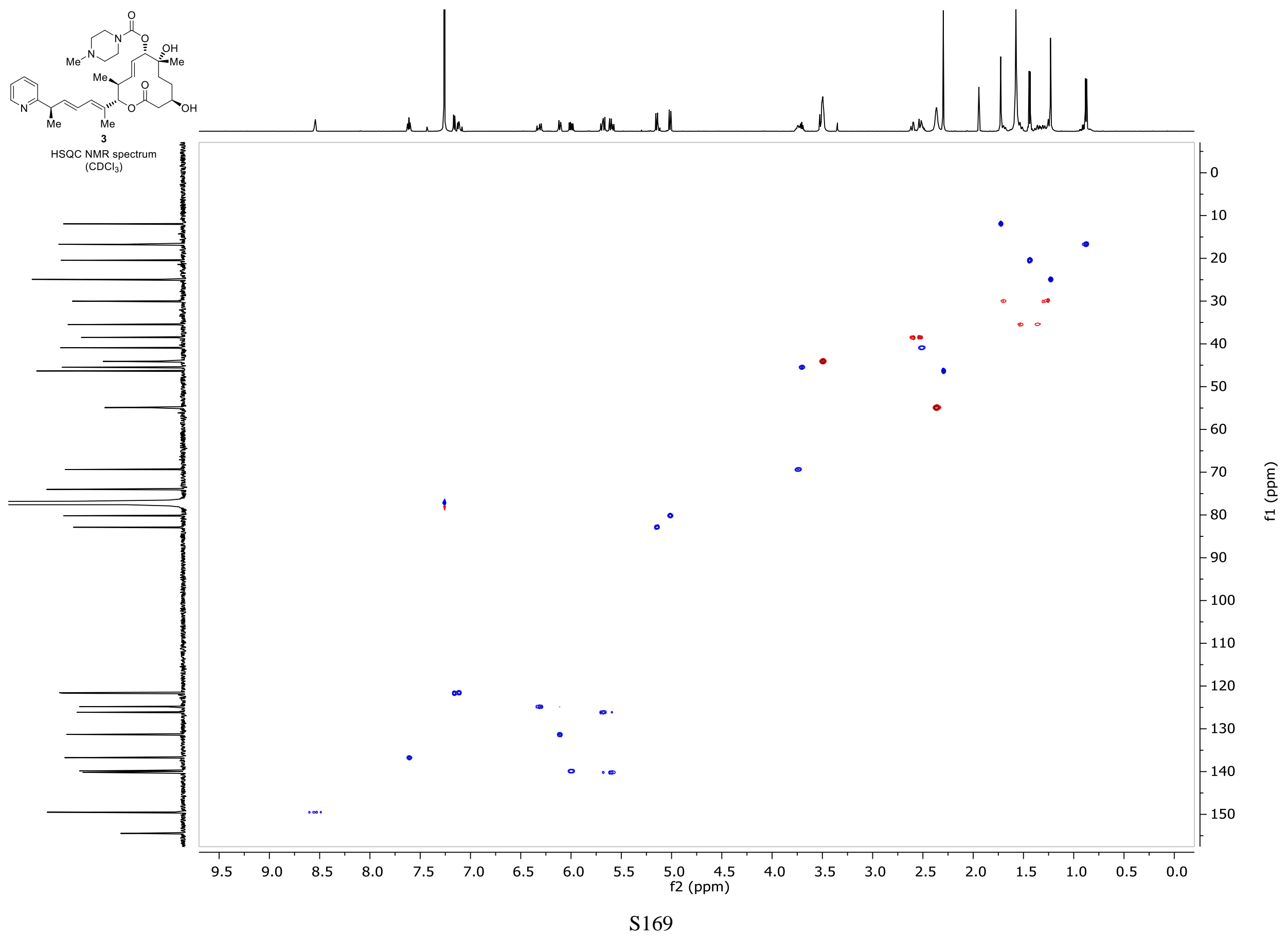


D. Rhoades, ${ }^{*}$ et al.

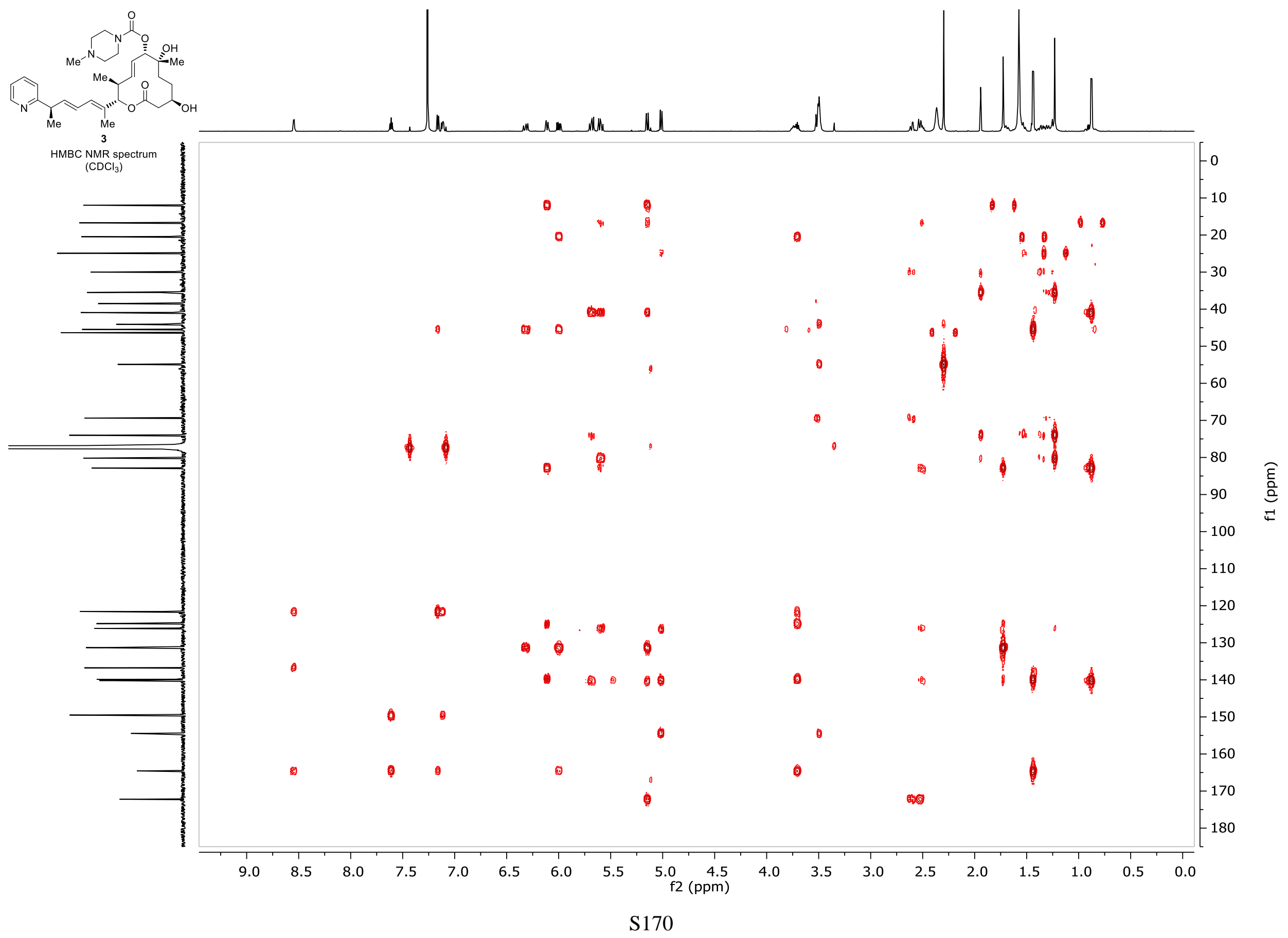


D. Rhoades, * et al.

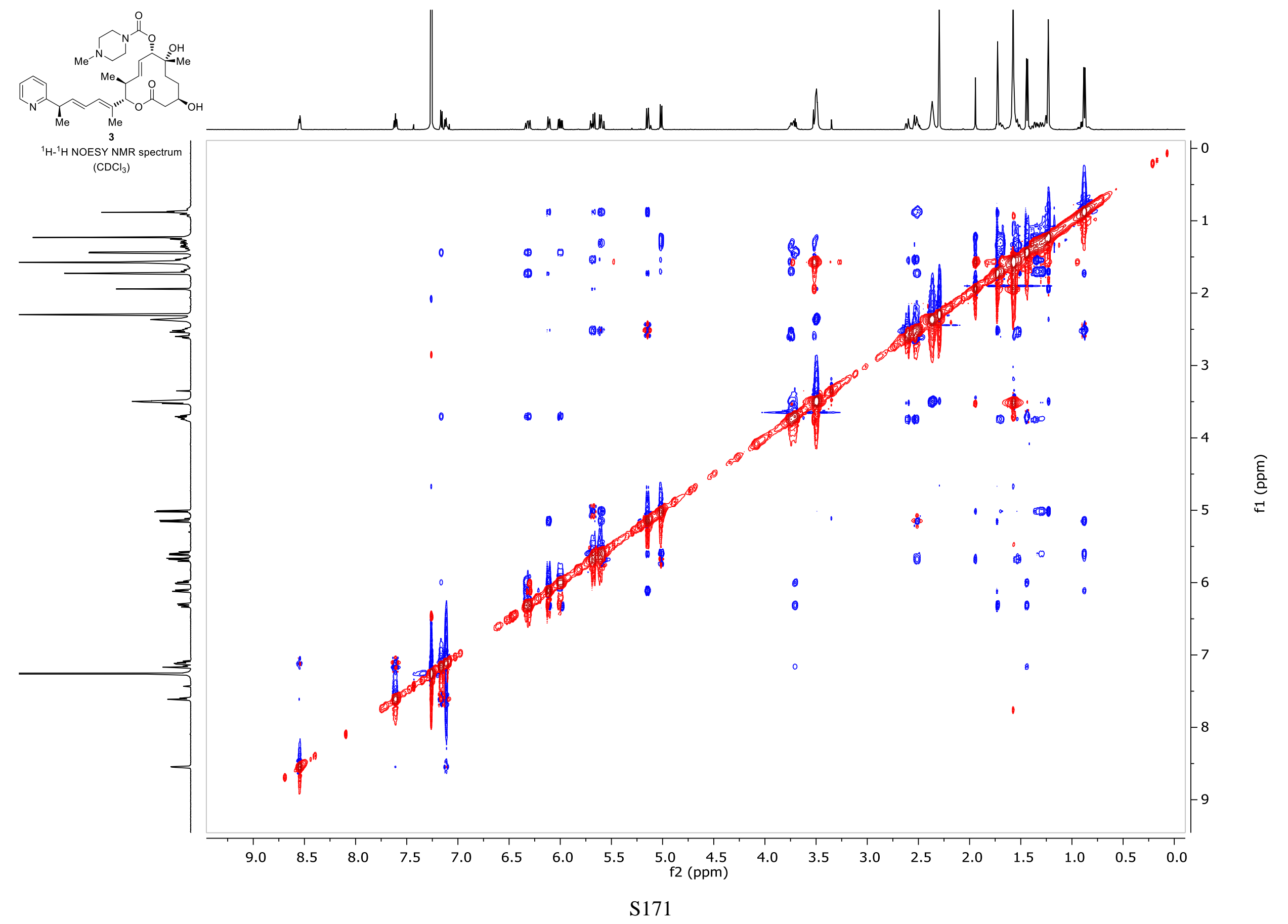




\section{Rhoades, * et al.}

\section{X-Ray Crystal Structure Data for Macrolide 20 and Macrolide 24}

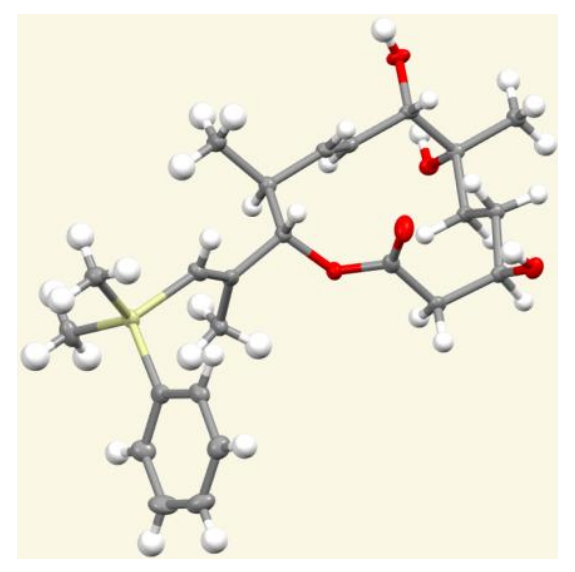

Table S13. Crystal data and structure refinement for 20.

Identification code

Empirical formula

Formula weight

Temperature

Wavelength

Crystal system

Space group

Unit cell dimensions

Volume

$\mathrm{Z}$

Density (calculated)

Absorption coefficient

$\mathrm{F}(000)$

Crystal size

Theta range for data collection

Index ranges

Reflections collected

Independent reflections

Completeness to theta $=67.679^{\circ}$

Absorption correction

Max. and min. transmission

Refinement method

Data/restraints/parameters

Goodness-of-fit on $\mathrm{F}^{2}$

Final R indices [I>2sigma(I)]

$\mathrm{R}$ indices (all data)

Absolute structure parameter

Extinction coefficient

Largest diff. peak and hole
DR201

C24 H36 O5 Si

432.62

$200 \mathrm{~K}$

$1.54178 \AA$

Monoclinic

P 21

$a=8.6311(2) \AA \quad \alpha=90^{\circ}$.

$\mathrm{b}=10.8502(2) \AA \quad \beta=106.9970(10)^{\circ}$.

$\mathrm{c}=13.4886(3) \AA \quad \gamma=90^{\circ}$.

$1208.02(5) \AA^{3}$

2

$1.189 \mathrm{Mg} / \mathrm{m}^{3}$

$1.104 \mathrm{~mm}^{-1}$

468

$0.23 \times 0.21 \times 0.06 \mathrm{~mm}^{3}$

3.426 to $68.948^{\circ}$.

$-10<=\mathrm{h}<=10,-13<=\mathrm{k}<=13,-16<=\mathrm{l}<=16$

19938

$4449[\mathrm{R}$ (int) $=0.0375]$

$100.0 \%$

Semi-empirical from equivalents

0.7532 and 0.6876

Full-matrix least-squares on $\mathrm{F}^{2}$

4449 / 1 / 279

1.087

$\mathrm{R} 1=0.0310, \mathrm{wR} 2=0.0773$

$\mathrm{R} 1=0.0326, \mathrm{wR} 2=0.0782$

$0.030(9)$

$\mathrm{n} / \mathrm{a}$

0.388 and -0.197 e. $\AA^{-3}$ 


\section{Rhoades, * et al.}

Table S14. Atomic coordinates (x 104) and equivalent isotropic displacement parameters $\left(\AA^{2} \times 10^{3}\right)$ for 20. $U(e q)$ is defined as one third of the trace of the orthogonalized $U^{i j}$ tensor.

\begin{tabular}{|c|c|c|c|c|}
\hline & $\mathrm{X}$ & $\mathrm{y}$ & $\mathrm{z}$ & $\mathrm{U}(\mathrm{eq})$ \\
\hline $\operatorname{Si}(1)$ & $1246(1)$ & $6559(1)$ & $4096(1)$ & $18(1)$ \\
\hline $\mathrm{O}(5)$ & $3978(2)$ & $3854(2)$ & $7308(1)$ & $18(1)$ \\
\hline $\mathrm{O}(2)$ & $2926(2)$ & $2506(2)$ & 10104(1) & 19(1) \\
\hline $\mathrm{O}(3)$ & $9658(2)$ & $1780(2)$ & $9522(1)$ & $16(1)$ \\
\hline $\mathrm{O}(4)$ & $10449(2)$ & $4208(2)$ & 10174(1) & $21(1)$ \\
\hline $\mathrm{O}(1)$ & $3306(3)$ & $4560(2)$ & $8703(2)$ & $27(1)$ \\
\hline $\mathrm{C}(3)$ & $3987(3)$ & $2057(2)$ & $9548(2)$ & $16(1)$ \\
\hline$C(14)$ & $3318(3)$ & $5186(2)$ & $5830(2)$ & $18(1)$ \\
\hline $\mathrm{C}(1)$ & $3529(3)$ & $3723(2)$ & $8173(2)$ & $18(1)$ \\
\hline $\mathrm{C}(8)$ & $8058(3)$ & $4130(2)$ & $8682(2)$ & $16(1)$ \\
\hline$C(12)$ & $9372(3)$ & $2213(2)$ & $11213(2)$ & $18(1)$ \\
\hline$C(6)$ & $8700(3)$ & $2453(2)$ & $10055(2)$ & $14(1)$ \\
\hline $\mathrm{C}(7)$ & $8780(3)$ & $3846(2)$ & $9814(2)$ & $16(1)$ \\
\hline $\mathrm{C}(9)$ & $6927(3)$ & $4977(2)$ & $8323(2)$ & $16(1)$ \\
\hline $\mathrm{C}(11)$ & $4253(3)$ & $5093(2)$ & $6968(2)$ & $16(1)$ \\
\hline $\mathrm{C}(4)$ & $5720(3)$ & $2493(2)$ & $10074(2)$ & $16(1)$ \\
\hline$C(10)$ & $6110(3)$ & $5252(2)$ & $7197(2)$ & $17(1)$ \\
\hline $\mathrm{C}(20)$ & $-734(3)$ & $5699(2)$ & $3801(2)$ & $19(1)$ \\
\hline$C(13)$ & $6502(3)$ & $6560(3)$ & $6917(2)$ & $25(1)$ \\
\hline $\mathrm{C}(2)$ & $3354(3)$ & $2372(2)$ & $8394(2)$ & $18(1)$ \\
\hline$C(5)$ & $6979(3)$ & $1945(2)$ & $9605(2)$ & $15(1)$ \\
\hline $\mathrm{C}(21)$ & $-1811(3)$ & $5990(2)$ & $4367(2)$ & $22(1)$ \\
\hline$C(16)$ & $2480(3)$ & $6202(2)$ & $5461(2)$ & $20(1)$ \\
\hline$C(22)$ & $-3341(4)$ & $5463(3)$ & $4131(2)$ & $26(1)$ \\
\hline$C(19)$ & $-1239(3)$ & $4841(3)$ & $3011(2)$ & $25(1)$ \\
\hline$C(15)$ & $3439(3)$ & $4082(3)$ & $5188(2)$ & $24(1)$ \\
\hline$C(23)$ & $-3812(3)$ & $4617(3)$ & $3339(3)$ & $30(1)$ \\
\hline $\mathrm{C}(24)$ & $-2761(4)$ & $4302(3)$ & $2782(2)$ & $31(1)$ \\
\hline$C(18)$ & $759(3)$ & $8240(3)$ & $4048(2)$ & $25(1)$ \\
\hline$C(17)$ & $2276(3)$ & $6204(3)$ & $3088(2)$ & $28(1)$ \\
\hline
\end{tabular}




\section{Rhoades, * et al.}

Table S15. Bond lengths $[\AA]$ and angles $\left[^{\circ}\right]$ for 20.

\begin{tabular}{ll}
\hline $\mathrm{Si}(1)-\mathrm{C}(20)$ & $1.885(3)$ \\
$\mathrm{Si}(1)-\mathrm{C}(16)$ & $1.878(3)$ \\
$\mathrm{Si}(1)-\mathrm{C}(18)$ & $1.869(3)$ \\
$\mathrm{Si}(1)-\mathrm{C}(17)$ & $1.869(3)$ \\
$\mathrm{O}(5)-\mathrm{C}(1)$ & $1.341(3)$ \\
$\mathrm{O}(5)-\mathrm{C}(11)$ & $1.462(3)$ \\
$\mathrm{O}(2)-\mathrm{H}(2)$ & 0.8400 \\
$\mathrm{O}(2)-\mathrm{C}(3)$ & $1.428(3)$ \\
$\mathrm{O}(3)-\mathrm{H}(3)$ & 0.8400 \\
$\mathrm{O}(3)-\mathrm{C}(6)$ & $1.443(3)$ \\
$\mathrm{O}(4)-\mathrm{H}(4)$ & 0.8400 \\
$\mathrm{O}(4)-\mathrm{C}(7)$ & $1.434(3)$ \\
$\mathrm{O}(1)-\mathrm{C}(1)$ & $1.206(3)$ \\
$\mathrm{C}(3)-\mathrm{H}(3 \mathrm{~A})$ & 1.0000 \\
$\mathrm{C}(3)-\mathrm{C}(4)$ & $1.531(3)$ \\
$\mathrm{C}(3)-\mathrm{C}(2)$ & $1.530(3)$ \\
$\mathrm{C}(14)-\mathrm{C}(11)$ & $1.514(3)$ \\
$\mathrm{C}(14)-\mathrm{C}(16)$ & $1.333(4)$ \\
$\mathrm{C}(14)-\mathrm{C}(15)$ & $1.500(4)$ \\
$\mathrm{C}(1)-\mathrm{C}(2)$ & $1.511(4)$ \\
$\mathrm{C}(8)-\mathrm{H}(8)$ & 0.9500 \\
$\mathrm{C}(8)-\mathrm{C}(7)$ & $1.503(3)$ \\
$\mathrm{C}(8)-\mathrm{C}(9)$ & $1.325(4)$ \\
$\mathrm{C}(12)-\mathrm{H}(12 \mathrm{~A})$ & 0.9800 \\
$\mathrm{C}(12)-\mathrm{H}(12 \mathrm{~B})$ & 0.9800 \\
$\mathrm{C}(12)-\mathrm{H}(12 \mathrm{C})$ & 0.9800 \\
$\mathrm{C}(12)-\mathrm{C}(6)$ & $1.521(3)$ \\
$\mathrm{C}(6)-\mathrm{C}(7)$ & $1.552(3)$ \\
$\mathrm{C}(6)-\mathrm{C}(5)$ & $1.533(3)$ \\
$\mathrm{C}(7)-\mathrm{H}(7)$ & 1.0000 \\
$\mathrm{C}(9)-\mathrm{H}(9)$ & 0.9500 \\
$\mathrm{C}(9)-\mathrm{C}(10)$ & $1.505(3)$ \\
$\mathrm{C}(11)-\mathrm{H}(11)$ & 1.0000 \\
$\mathrm{C}(11)-\mathrm{C}(10)$ & $1.550(3)$ \\
$\mathrm{C}(4)-\mathrm{H}(4 \mathrm{~A})$ & \\
& 0.9900 \\
&
\end{tabular}



D. Rhoades, * et al.

\begin{tabular}{|c|c|}
\hline $\mathrm{C}(4)-\mathrm{H}(4 \mathrm{~B})$ & 0.9900 \\
\hline$C(4)-C(5)$ & $1.529(3)$ \\
\hline $\mathrm{C}(10)-\mathrm{H}(10)$ & 1.0000 \\
\hline$C(10)-C(13)$ & $1.531(4)$ \\
\hline$C(20)-C(21)$ & $1.401(4)$ \\
\hline$C(20)-C(19)$ & $1.386(4)$ \\
\hline $\mathrm{C}(13)-\mathrm{H}(13 \mathrm{~A})$ & 0.9800 \\
\hline $\mathrm{C}(13)-\mathrm{H}(13 \mathrm{~B})$ & 0.9800 \\
\hline $\mathrm{C}(13)-\mathrm{H}(13 \mathrm{C})$ & 0.9800 \\
\hline $\mathrm{C}(2)-\mathrm{H}(2 \mathrm{~A})$ & 0.9900 \\
\hline $\mathrm{C}(2)-\mathrm{H}(2 \mathrm{~B})$ & 0.9900 \\
\hline $\mathrm{C}(5)-\mathrm{H}(5 \mathrm{~A})$ & 0.9900 \\
\hline $\mathrm{C}(5)-\mathrm{H}(5 \mathrm{~B})$ & 0.9900 \\
\hline $\mathrm{C}(21)-\mathrm{H}(21)$ & 0.9500 \\
\hline$C(21)-C(22)$ & $1.388(4)$ \\
\hline $\mathrm{C}(16)-\mathrm{H}(16)$ & 0.9500 \\
\hline $\mathrm{C}(22)-\mathrm{H}(22)$ & 0.9500 \\
\hline$C(22)-C(23)$ & $1.377(4)$ \\
\hline $\mathrm{C}(19)-\mathrm{H}(19)$ & 0.9500 \\
\hline$C(19)-C(24)$ & $1.387(4)$ \\
\hline $\mathrm{C}(15)-\mathrm{H}(15 \mathrm{~A})$ & 0.9800 \\
\hline $\mathrm{C}(15)-\mathrm{H}(15 \mathrm{~B})$ & 0.9800 \\
\hline $\mathrm{C}(15)-\mathrm{H}(15 \mathrm{C})$ & 0.9800 \\
\hline $\mathrm{C}(23)-\mathrm{H}(23)$ & 0.9500 \\
\hline $\mathrm{C}(23)-\mathrm{C}(24)$ & $1.380(4)$ \\
\hline $\mathrm{C}(24)-\mathrm{H}(24)$ & 0.9500 \\
\hline $\mathrm{C}(18)-\mathrm{H}(18 \mathrm{~A})$ & 0.9800 \\
\hline $\mathrm{C}(18)-\mathrm{H}(18 \mathrm{~B})$ & 0.9800 \\
\hline $\mathrm{C}(18)-\mathrm{H}(18 \mathrm{C})$ & 0.9800 \\
\hline $\mathrm{C}(17)-\mathrm{H}(17 \mathrm{~A})$ & 0.9800 \\
\hline $\mathrm{C}(17)-\mathrm{H}(17 \mathrm{~B})$ & 0.9800 \\
\hline $\mathrm{C}(17)-\mathrm{H}(17 \mathrm{C})$ & 0.9800 \\
\hline$C(16)-S i(1)-C(20)$ & $108.91(12)$ \\
\hline$C(18)-S i(1)-C(20)$ & $107.18(12)$ \\
\hline$C(18)-S i(1)-C(16)$ & $107.00(12)$ \\
\hline$C(18)-S i(1)-C(17)$ & $109.04(14)$ \\
\hline $\mathrm{C}(17)-\mathrm{Si}(1)-\mathrm{C}(20)$ & $109.83(13)$ \\
\hline
\end{tabular}




\section{Rhoades, * et al.}

$\begin{array}{ll}\mathrm{C}(17)-\mathrm{Si}(1)-\mathrm{C}(16) & 114.59(12) \\ \mathrm{C}(1)-\mathrm{O}(5)-\mathrm{C}(11) & 119.0(2) \\ \mathrm{C}(3)-\mathrm{O}(2)-\mathrm{H}(2) & 109.5 \\ \mathrm{C}(6)-\mathrm{O}(3)-\mathrm{H}(3) & 109.5 \\ \mathrm{C}(7)-\mathrm{O}(4)-\mathrm{H}(4) & 109.5 \\ \mathrm{O}(2)-\mathrm{C}(3)-\mathrm{H}(3 \mathrm{~A}) & 106.9 \\ \mathrm{O}(2)-\mathrm{C}(3)-\mathrm{C}(4) & 109.9(2) \\ \mathrm{O}(2)-\mathrm{C}(3)-\mathrm{C}(2) & 111.4(2) \\ \mathrm{C}(4)-\mathrm{C}(3)-\mathrm{H}(3 \mathrm{~A}) & 106.9 \\ \mathrm{C}(2)-\mathrm{C}(3)-\mathrm{H}(3 \mathrm{~A}) & 106.9 \\ \mathrm{C}(2)-\mathrm{C}(3)-\mathrm{C}(4) & 114.4(2) \\ \mathrm{C}(16)-\mathrm{C}(14)-\mathrm{C}(11) & 120.3(2) \\ \mathrm{C}(16)-\mathrm{C}(14)-\mathrm{C}(15) & 124.5(2) \\ \mathrm{C}(15)-\mathrm{C}(14)-\mathrm{C}(11) & 115.2(2) \\ \mathrm{O}(5)-\mathrm{C}(1)-\mathrm{C}(2) & 110.2(2) \\ \mathrm{O}(1)-\mathrm{C}(1)-\mathrm{O}(5) & 124.9(2) \\ \mathrm{O}(1)-\mathrm{C}(1)-\mathrm{C}(2) & 124.8(2) \\ \mathrm{C}(7)-\mathrm{C}(8)-\mathrm{H}(8) & 118.1 \\ \mathrm{C}(9)-\mathrm{C}(8)-\mathrm{H}(8) & 118.1 \\ \mathrm{C}(9)-\mathrm{C}(8)-\mathrm{C}(7) & 123.8(2) \\ \mathrm{H}(12 \mathrm{~A})-\mathrm{C}(12)-\mathrm{H}(12 \mathrm{~B}) & 109.5 \\ \mathrm{H}(12 \mathrm{~A})-\mathrm{C}(12)-\mathrm{H}(12 \mathrm{C}) & 109.5 \\ \mathrm{H}(12 \mathrm{~B})-\mathrm{C}(12)-\mathrm{H}(12 \mathrm{C}) & 109.5 \\ \mathrm{C}(6)-\mathrm{C}(12)-\mathrm{H}(12 \mathrm{~A}) & 109.5 \\ \mathrm{C}(6)-\mathrm{C}(12)-\mathrm{H}(12 \mathrm{~B}) & 109.5 \\ \mathrm{C}(6)-\mathrm{C}(12)-\mathrm{H}(12 \mathrm{C}) & 109.5 \\ \mathrm{O}(3)-\mathrm{C}(6)-\mathrm{C}(12) & 108.81(18) \\ \mathrm{O}(3)-\mathrm{C}(6)-\mathrm{C}(7) & 108.78(19) \\ \mathrm{O}(3)-\mathrm{C}(6)-\mathrm{C}(5) & 104.55(18) \\ \mathrm{C}(12)-\mathrm{C}(6)-\mathrm{C}(7) & 110.8(2) \\ \mathrm{C}(12)-\mathrm{C}(6)-\mathrm{C}(5) & 112.31(19) \\ \mathrm{C}(5)-\mathrm{C}(6)-\mathrm{C}(7) & 111.35(19) \\ \mathrm{O}(4)-\mathrm{C}(7)-\mathrm{C}(8) & 111.4(2) \\ \mathrm{O}(4)-\mathrm{C}(7)-\mathrm{C}(6) & 107.31(18) \\ \mathrm{O}(4)-\mathrm{C}(7)-\mathrm{H}(7) & 108.5 \\ \mathrm{C}(8)-\mathrm{C}(7)-\mathrm{C}(6) & 112.4(2) \\ \mathrm{C}(8)-\mathrm{C}(7)-\mathrm{H}(7) & 108.5 \\ & \end{array}$




\section{Rhoades, * et al.}

\begin{tabular}{|c|c|}
\hline $\mathrm{C}(6)-\mathrm{C}(7)-\mathrm{H}(7)$ & 108.5 \\
\hline $\mathrm{C}(8)-\mathrm{C}(9)-\mathrm{H}(9)$ & 117.1 \\
\hline$C(8)-C(9)-C(10)$ & $125.7(2)$ \\
\hline $\mathrm{C}(10)-\mathrm{C}(9)-\mathrm{H}(9)$ & 117.1 \\
\hline $\mathrm{O}(5)-\mathrm{C}(11)-\mathrm{C}(14)$ & $106.96(19)$ \\
\hline $\mathrm{O}(5)-\mathrm{C}(11)-\mathrm{H}(11)$ & 109.6 \\
\hline $\mathrm{O}(5)-\mathrm{C}(11)-\mathrm{C}(10)$ & 107.01(19) \\
\hline $\mathrm{C}(14)-\mathrm{C}(11)-\mathrm{H}(11)$ & 109.6 \\
\hline$C(14)-C(11)-C(10)$ & $114.1(2)$ \\
\hline $\mathrm{C}(10)-\mathrm{C}(11)-\mathrm{H}(11)$ & 109.6 \\
\hline $\mathrm{C}(3)-\mathrm{C}(4)-\mathrm{H}(4 \mathrm{~A})$ & 108.8 \\
\hline $\mathrm{C}(3)-\mathrm{C}(4)-\mathrm{H}(4 \mathrm{~B})$ & 108.8 \\
\hline $\mathrm{H}(4 \mathrm{~A})-\mathrm{C}(4)-\mathrm{H}(4 \mathrm{~B})$ & 107.7 \\
\hline$C(5)-C(4)-C(3)$ & $113.8(2)$ \\
\hline $\mathrm{C}(5)-\mathrm{C}(4)-\mathrm{H}(4 \mathrm{~A})$ & 108.8 \\
\hline $\mathrm{C}(5)-\mathrm{C}(4)-\mathrm{H}(4 \mathrm{~B})$ & 108.8 \\
\hline C(9)-C(10)-C(11) & $109.4(2)$ \\
\hline $\mathrm{C}(9)-\mathrm{C}(10)-\mathrm{H}(10)$ & 108.7 \\
\hline C(9)-C(10)-C(13) & $111.1(2)$ \\
\hline $\mathrm{C}(11)-\mathrm{C}(10)-\mathrm{H}(10)$ & 108.7 \\
\hline $\mathrm{C}(13)-\mathrm{C}(10)-\mathrm{C}(11)$ & $110.1(2)$ \\
\hline $\mathrm{C}(13)-\mathrm{C}(10)-\mathrm{H}(10)$ & 108.7 \\
\hline $\mathrm{C}(21)-\mathrm{C}(20)-\mathrm{Si}(1)$ & $118.6(2)$ \\
\hline$C(19)-C(20)-S i(1)$ & $123.8(2)$ \\
\hline $\mathrm{C}(19)-\mathrm{C}(20)-\mathrm{C}(21)$ & $117.5(2)$ \\
\hline $\mathrm{C}(10)-\mathrm{C}(13)-\mathrm{H}(13 \mathrm{~A})$ & 109.5 \\
\hline $\mathrm{C}(10)-\mathrm{C}(13)-\mathrm{H}(13 \mathrm{~B})$ & 109.5 \\
\hline $\mathrm{C}(10)-\mathrm{C}(13)-\mathrm{H}(13 \mathrm{C})$ & 109.5 \\
\hline $\mathrm{H}(13 \mathrm{~A})-\mathrm{C}(13)-\mathrm{H}(13 \mathrm{~B})$ & 109.5 \\
\hline $\mathrm{H}(13 \mathrm{~A})-\mathrm{C}(13)-\mathrm{H}(13 \mathrm{C})$ & 109.5 \\
\hline $\mathrm{H}(13 \mathrm{~B})-\mathrm{C}(13)-\mathrm{H}(13 \mathrm{C})$ & 109.5 \\
\hline $\mathrm{C}(3)-\mathrm{C}(2)-\mathrm{H}(2 \mathrm{~A})$ & 109.0 \\
\hline $\mathrm{C}(3)-\mathrm{C}(2)-\mathrm{H}(2 \mathrm{~B})$ & 109.0 \\
\hline $\mathrm{C}(1)-\mathrm{C}(2)-\mathrm{C}(3)$ & $113.0(2)$ \\
\hline $\mathrm{C}(1)-\mathrm{C}(2)-\mathrm{H}(2 \mathrm{~A})$ & 109.0 \\
\hline $\mathrm{C}(1)-\mathrm{C}(2)-\mathrm{H}(2 \mathrm{~B})$ & 109.0 \\
\hline $\mathrm{H}(2 \mathrm{~A})-\mathrm{C}(2)-\mathrm{H}(2 \mathrm{~B})$ & 107.8 \\
\hline
\end{tabular}




\section{Rhoades, * et al.}

\begin{tabular}{|c|c|}
\hline$C(6)-C(5)-H(5 A)$ & 108.6 \\
\hline $\mathrm{C}(6)-\mathrm{C}(5)-\mathrm{H}(5 \mathrm{~B})$ & 108.6 \\
\hline$C(4)-C(5)-C(6)$ & $114.85(19)$ \\
\hline $\mathrm{C}(4)-\mathrm{C}(5)-\mathrm{H}(5 \mathrm{~A})$ & 108.6 \\
\hline $\mathrm{C}(4)-\mathrm{C}(5)-\mathrm{H}(5 \mathrm{~B})$ & 108.6 \\
\hline $\mathrm{H}(5 \mathrm{~A})-\mathrm{C}(5)-\mathrm{H}(5 \mathrm{~B})$ & 107.5 \\
\hline $\mathrm{C}(20)-\mathrm{C}(21)-\mathrm{H}(21)$ & 119.3 \\
\hline $\mathrm{C}(22)-\mathrm{C}(21)-\mathrm{C}(20)$ & $121.3(3)$ \\
\hline $\mathrm{C}(22)-\mathrm{C}(21)-\mathrm{H}(21)$ & 119.3 \\
\hline $\mathrm{Si}(1)-\mathrm{C}(16)-\mathrm{H}(16)$ & 115.6 \\
\hline $\mathrm{C}(14)-\mathrm{C}(16)-\mathrm{Si}(1)$ & $128.9(2)$ \\
\hline $\mathrm{C}(14)-\mathrm{C}(16)-\mathrm{H}(16)$ & 115.6 \\
\hline $\mathrm{C}(21)-\mathrm{C}(22)-\mathrm{H}(22)$ & 120.0 \\
\hline $\mathrm{C}(23)-\mathrm{C}(22)-\mathrm{C}(21)$ & $119.9(3)$ \\
\hline $\mathrm{C}(23)-\mathrm{C}(22)-\mathrm{H}(22)$ & 120.0 \\
\hline $\mathrm{C}(20)-\mathrm{C}(19)-\mathrm{H}(19)$ & 119.4 \\
\hline $\mathrm{C}(20)-\mathrm{C}(19)-\mathrm{C}(24)$ & 121.3(3) \\
\hline $\mathrm{C}(24)-\mathrm{C}(19)-\mathrm{H}(19)$ & 119.4 \\
\hline $\mathrm{C}(14)-\mathrm{C}(15)-\mathrm{H}(15 \mathrm{~A})$ & 109.5 \\
\hline $\mathrm{C}(14)-\mathrm{C}(15)-\mathrm{H}(15 \mathrm{~B})$ & 109.5 \\
\hline $\mathrm{C}(14)-\mathrm{C}(15)-\mathrm{H}(15 \mathrm{C})$ & 109.5 \\
\hline $\mathrm{H}(15 \mathrm{~A})-\mathrm{C}(15)-\mathrm{H}(15 \mathrm{~B})$ & 109.5 \\
\hline $\mathrm{H}(15 \mathrm{~A})-\mathrm{C}(15)-\mathrm{H}(15 \mathrm{C})$ & 109.5 \\
\hline $\mathrm{H}(15 \mathrm{~B})-\mathrm{C}(15)-\mathrm{H}(15 \mathrm{C})$ & 109.5 \\
\hline $\mathrm{C}(22)-\mathrm{C}(23)-\mathrm{H}(23)$ & 120.2 \\
\hline$C(22)-C(23)-C(24)$ & $119.7(3)$ \\
\hline $\mathrm{C}(24)-\mathrm{C}(23)-\mathrm{H}(23)$ & 120.2 \\
\hline $\mathrm{C}(19)-\mathrm{C}(24)-\mathrm{H}(24)$ & 119.8 \\
\hline $\mathrm{C}(23)-\mathrm{C}(24)-\mathrm{C}(19)$ & $120.3(3)$ \\
\hline $\mathrm{C}(23)-\mathrm{C}(24)-\mathrm{H}(24)$ & 119.8 \\
\hline $\mathrm{Si}(1)-\mathrm{C}(18)-\mathrm{H}(18 \mathrm{~A})$ & 109.5 \\
\hline $\mathrm{Si}(1)-\mathrm{C}(18)-\mathrm{H}(18 \mathrm{~B})$ & 109.5 \\
\hline $\mathrm{Si}(1)-\mathrm{C}(18)-\mathrm{H}(18 \mathrm{C})$ & 109.5 \\
\hline $\mathrm{H}(18 \mathrm{~A})-\mathrm{C}(18)-\mathrm{H}(18 \mathrm{~B})$ & 109.5 \\
\hline $\mathrm{H}(18 \mathrm{~A})-\mathrm{C}(18)-\mathrm{H}(18 \mathrm{C})$ & 109.5 \\
\hline $\mathrm{H}(18 \mathrm{~B})-\mathrm{C}(18)-\mathrm{H}(18 \mathrm{C})$ & 109.5 \\
\hline $\mathrm{Si}(1)-\mathrm{C}(17)-\mathrm{H}(17 \mathrm{~A})$ & 109.5 \\
\hline
\end{tabular}




\section{Rhoades, * et al.}

$\mathrm{Si}(1)-\mathrm{C}(17)-\mathrm{H}(17 \mathrm{~B}) \quad 109.5$

$\mathrm{Si}(1)-\mathrm{C}(17)-\mathrm{H}(17 \mathrm{C}) \quad 109.5$

$\mathrm{H}(17 \mathrm{~A})-\mathrm{C}(17)-\mathrm{H}(17 \mathrm{~B}) \quad 109.5$

$\mathrm{H}(17 \mathrm{~A})-\mathrm{C}(17)-\mathrm{H}(17 \mathrm{C}) \quad 109.5$

H(17B)-C(17)-H(17C) 109.5

Symmetry transformations used to generate equivalent atoms:

Table S16. Anisotropic displacement parameters $\left(\AA^{2} \times 10^{3}\right)$ for 20. The anisotropic displacement factor exponent takes the form: $-2 \pi^{2}\left[h^{2} a^{* 2} U^{11}+\ldots+2 h k a^{*} b^{*} U^{12}\right]$

\begin{tabular}{|c|c|c|c|c|c|c|}
\hline & $\mathrm{U}^{11}$ & $\mathrm{U}^{22}$ & $\mathrm{U}^{33}$ & $\mathrm{U}^{23}$ & $\mathrm{U}^{13}$ & $\mathrm{U}^{12}$ \\
\hline $\operatorname{Si}(1)$ & $14(1)$ & $22(1)$ & $15(1)$ & $2(1)$ & 1(1) & $2(1)$ \\
\hline $\mathrm{O}(5)$ & $20(1)$ & $18(1)$ & $14(1)$ & $2(1)$ & $4(1)$ & $-2(1)$ \\
\hline $\mathrm{O}(2)$ & $14(1)$ & $21(1)$ & $24(1)$ & $4(1)$ & $9(1)$ & $2(1)$ \\
\hline $\mathrm{O}(3)$ & $11(1)$ & $16(1)$ & $20(1)$ & $0(1)$ & $5(1)$ & $2(1)$ \\
\hline $\mathrm{O}(4)$ & $12(1)$ & $17(1)$ & $30(1)$ & $0(1)$ & $2(1)$ & $-2(1)$ \\
\hline $\mathrm{O}(1)$ & $36(1)$ & $23(1)$ & $28(1)$ & $4(1)$ & $18(1)$ & $6(1)$ \\
\hline $\mathrm{C}(3)$ & $12(1)$ & $18(1)$ & $19(1)$ & $1(1)$ & $6(1)$ & $-1(1)$ \\
\hline$C(14)$ & $15(1)$ & $22(1)$ & $16(1)$ & $1(1)$ & $3(1)$ & $-6(1)$ \\
\hline $\mathrm{C}(1)$ & $13(1)$ & $23(1)$ & $16(1)$ & $3(1)$ & $3(1)$ & $1(1)$ \\
\hline $\mathrm{C}(8)$ & $15(1)$ & $14(1)$ & $21(1)$ & $-1(1)$ & $7(1)$ & $-3(1)$ \\
\hline$C(12)$ & $14(1)$ & $20(1)$ & $18(1)$ & $1(1)$ & $2(1)$ & $1(1)$ \\
\hline$C(6)$ & 11(1) & $14(1)$ & $18(1)$ & $-1(1)$ & $5(1)$ & $2(1)$ \\
\hline$C(7)$ & $11(1)$ & $14(1)$ & $20(1)$ & $-2(1)$ & $2(1)$ & $-1(1)$ \\
\hline $\mathrm{C}(9)$ & $16(1)$ & $16(1)$ & $16(1)$ & $-2(1)$ & $3(1)$ & $-2(1)$ \\
\hline$C(11)$ & $18(1)$ & $16(1)$ & $15(1)$ & $1(1)$ & $4(1)$ & $-1(1)$ \\
\hline $\mathrm{C}(4)$ & $14(1)$ & $19(1)$ & $16(1)$ & $-1(1)$ & $4(1)$ & $0(1)$ \\
\hline$C(10)$ & $17(1)$ & $18(1)$ & $16(1)$ & $1(1)$ & $3(1)$ & $0(1)$ \\
\hline$C(20)$ & $17(1)$ & $17(1)$ & $20(1)$ & $5(1)$ & $0(1)$ & $4(1)$ \\
\hline$C(13)$ & $24(1)$ & $23(1)$ & $22(1)$ & $6(1)$ & $-1(1)$ & $-5(1)$ \\
\hline $\mathrm{C}(2)$ & $12(1)$ & $23(1)$ & $19(1)$ & $2(1)$ & $3(1)$ & $-5(1)$ \\
\hline$C(5)$ & $12(1)$ & $15(1)$ & $16(1)$ & $1(1)$ & $3(1)$ & $0(1)$ \\
\hline$C(21)$ & $24(1)$ & $19(1)$ & $19(1)$ & 1(1) & $3(1)$ & $4(1)$ \\
\hline$C(16)$ & $18(1)$ & $26(1)$ & $15(1)$ & $-1(1)$ & $3(1)$ & $-2(1)$ \\
\hline $\mathrm{C}(22)$ & $24(1)$ & $23(1)$ & $33(2)$ & $2(1)$ & $11(1)$ & $3(1)$ \\
\hline
\end{tabular}


D. Rhoades, * et al.

$\begin{array}{lllllll}\mathrm{C}(19) & 22(1) & 25(1) & 29(1) & -4(1) & 6(1) & 5(1) \\ \mathrm{C}(15) & 32(1) & 22(1) & 16(1) & 3(1) & 3(1) & -5(1) \\ \mathrm{C}(23) & 21(1) & 22(1) & 44(2) & -1(1) & 5(1) & -3(1) \\ \mathrm{C}(24) & 29(2) & 24(1) & 37(2) & -12(1) & 4(1) & 0(1) \\ \mathrm{C}(18) & 23(1) & 22(1) & 27(1) & 2(1) & 3(1) & -2(1) \\ \mathrm{C}(17) & 23(1) & 41(2) & 20(1) & 7(1) & 7(1) & 7(1)\end{array}$

Table S17. Hydrogen coordinates (x 104) and isotropic displacement parameters $\left(\AA^{2} \times 10^{3}\right)$ for 20.

\begin{tabular}{|c|c|c|c|c|}
\hline & $\mathrm{X}$ & $\mathrm{y}$ & $\mathrm{z}$ & $\mathrm{U}(\mathrm{eq})$ \\
\hline $\mathrm{H}(2)$ & 2711 & 3250 & 9955 & 29 \\
\hline $\mathrm{H}(3)$ & 10611 & 2051 & 9703 & 24 \\
\hline $\mathrm{H}(4)$ & 10509 & 4977 & 10242 & 31 \\
\hline $\mathrm{H}(3 \mathrm{~A})$ & 3993 & 1137 & 9603 & 19 \\
\hline $\mathrm{H}(8)$ & 8435 & 3677 & 8196 & 20 \\
\hline $\mathrm{H}(12 \mathrm{~A})$ & 9276 & 1334 & 11350 & 27 \\
\hline $\mathrm{H}(12 \mathrm{~B})$ & 8759 & 2694 & 11585 & 27 \\
\hline $\mathrm{H}(12 \mathrm{C})$ & 10515 & 2455 & 11449 & 27 \\
\hline $\mathrm{H}(7)$ & 8165 & 4314 & 10215 & 19 \\
\hline $\mathrm{H}(9)$ & 6603 & 5452 & 8819 & 19 \\
\hline $\mathrm{H}(11)$ & 3833 & 5717 & 7370 & 20 \\
\hline $\mathrm{H}(4 \mathrm{~A})$ & 5755 & 3402 & 10027 & 20 \\
\hline $\mathrm{H}(4 \mathrm{~B})$ & 6015 & 2272 & 10818 & 20 \\
\hline $\mathrm{H}(10)$ & 6506 & 4652 & 6764 & 21 \\
\hline $\mathrm{H}(13 \mathrm{~A})$ & 6260 & 7148 & 7403 & 37 \\
\hline $\mathrm{H}(13 \mathrm{~B})$ & 5843 & 6755 & 6210 & 37 \\
\hline $\mathrm{H}(13 \mathrm{C})$ & 7653 & 6612 & 6959 & 37 \\
\hline $\mathrm{H}(2 \mathrm{~A})$ & 3952 & 1877 & 8010 & 22 \\
\hline $\mathrm{H}(2 \mathrm{~B})$ & 2196 & 2141 & 8135 & 22 \\
\hline $\mathrm{H}(5 \mathrm{~A})$ & 6626 & 2100 & 8849 & 18 \\
\hline $\mathrm{H}(5 \mathrm{~B})$ & 7008 & 1041 & 9709 & 18 \\
\hline $\mathrm{H}(21)$ & -1487 & 6560 & 4925 & 26 \\
\hline $\mathrm{H}(16)$ & 2512 & 6832 & 5955 & 24 \\
\hline $\mathrm{H}(22)$ & -4062 & 5687 & 4515 & 32 \\
\hline H(19) & -529 & 4617 & 2619 & 30 \\
\hline
\end{tabular}




\begin{tabular}{lrrrr} 
& \multicolumn{3}{c}{ D. Rhoades, * et al. } \\
$\mathrm{H}(15 \mathrm{~A})$ & 4528 & 4042 & 5103 & 37 \\
$\mathrm{H}(15 \mathrm{~B})$ & 2635 & 4149 & 4505 & 37 \\
$\mathrm{H}(15 \mathrm{C})$ & 3233 & 3333 & 5535 & 37 \\
$\mathrm{H}(23)$ & -4856 & 4251 & 3177 & 36 \\
$\mathrm{H}(24)$ & -3080 & 3714 & 2238 & 37 \\
$\mathrm{H}(18 \mathrm{~A})$ & 1766 & 8718 & 4216 & 38 \\
$\mathrm{H}(18 \mathrm{~B})$ & 174 & 8426 & 4553 & 38 \\
$\mathrm{H}(18 \mathrm{C})$ & 82 & 8458 & 3351 & 38 \\
$\mathrm{H}(17 \mathrm{~A})$ & 1573 & 6446 & 2404 & 42 \\
$\mathrm{H}(17 \mathrm{~B})$ & 2499 & 5318 & 3091 & 42 \\
$\mathrm{H}(17 \mathrm{C})$ & 3298 & 6662 & 3240 & 42 \\
\hline
\end{tabular}


D. Rhoades, * et al.

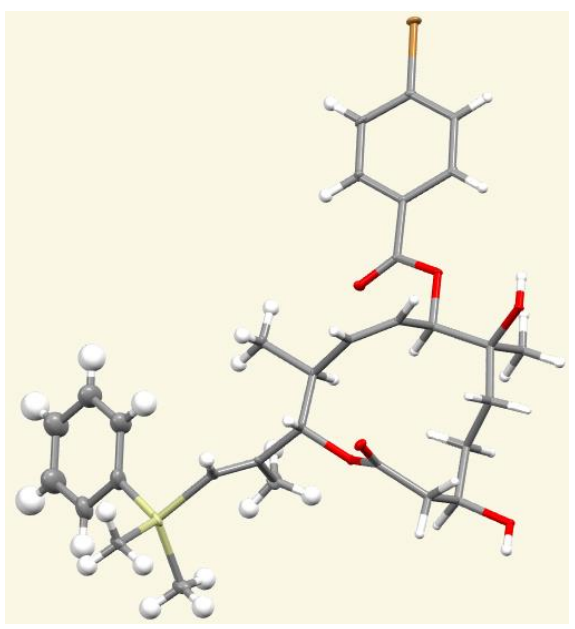

Table S18. Crystal data and structure refinement for $\mathbf{2 4}$.

Identification code

Empirical formula

Formula weight

Temperature/K

Crystal system

Space group

a/A

$\mathrm{b} / \AA$

$\mathrm{c} / \AA$

$\alpha /^{\circ}$

$\beta /{ }^{\circ}$

$\gamma /{ }^{\circ}$

Volume $/ \AA^{3}$

$\mathrm{Z}$

$\rho_{\text {calc }} \mathrm{g} / \mathrm{cm}^{3}$

$\mu / \mathrm{mm}^{-1}$

$\mathrm{F}(000)$

Crystal size $/ \mathrm{mm}^{3}$

Radiation

$2 \Theta$ range for data collection ${ }^{\circ}$

Index ranges

Reflections collected

Independent reflections

Data/restraints/parameters

Goodness-of-fit on $\mathrm{F}^{2}$

Final $\mathrm{R}$ indexes $[\mathrm{I}>=2 \sigma(\mathrm{I})]$

Final $\mathrm{R}$ indexes [all data]

Largest diff. peak/hole / e $\AA^{-3}$

Flack parameter
DR264

$\mathrm{C}_{31} \mathrm{H}_{39} \mathrm{BrO}_{6} \mathrm{Si}$

614.61

100.0

monoclinic

$\mathrm{C} 2$

$15.235(5)$

9.811(3)

21.302(6)

90

101.577(9)

90

3119.1(16)

4

1.309

1.396

1284.0

$0.33 \times 0.31 \times 0.04$

$\operatorname{MoK} \alpha(\lambda=0.71073)$

3.904 to 50.786

$-18 \leq \mathrm{h} \leq 18,-11 \leq \mathrm{k} \leq 11,-25 \leq 1 \leq 20$

7552

$5103\left[\mathrm{R}_{\text {int }}=0.0352, \mathrm{R}_{\text {sigma }}=0.1084\right]$

$5103 / 70 / 349$

1.019

$\mathrm{R}_{1}=0.0686, \mathrm{wR}_{2}=0.1682$

$\mathrm{R}_{1}=0.1011, \mathrm{wR}_{2}=0.1834$

$0.57 /-0.48$

$0.045(10)$ 


\section{Rhoades, * et al.}

Table S19. Fractional Atomic Coordinates $\left(\times 10^{4}\right)$ and Equivalent Isotropic Displacement Parameters $\left(\AA^{2} \times 10^{3}\right)$ for $\mathbf{2 4}$. $\mathrm{U}(\mathrm{eq})$ is defined as one third of the trace of the orthogonalized Uij tensor.

\begin{tabular}{|c|c|c|c|c|}
\hline Atom & $\mathrm{x}$ & $\mathrm{y}$ & $\mathrm{z}$ & $\mathrm{U}(\mathrm{eq})$ \\
\hline $\mathrm{Br} 1$ & $9632.1(6)$ & $4260.2(17)$ & $7213.1(6)$ & $54.1(4)$ \\
\hline Si1' & $1135(3)$ & $4499(6)$ & $9062(3)$ & $55.0(15)$ \\
\hline O5 & $5138(4)$ & $5087(6)$ & 6332(3) & $22.9(12)$ \\
\hline $\mathrm{O} 4$ & $4120(4)$ & $4808(6)$ & $5099(3)$ & $22.0(14)$ \\
\hline $\mathrm{O} 3$ & $920(4)$ & 7036(6) & $5103(3)$ & $23.9(15)$ \\
\hline $\mathrm{O} 2$ & 1791(4) & $4863(6)$ & $6824(3)$ & $26.2(15)$ \\
\hline $\mathrm{O} 1$ & 1321(4) & 2950(7) & $6267(3)$ & $34.6(17)$ \\
\hline O6 & $5257(4)$ & $5423(8)$ & 7393(4) & $38.9(18)$ \\
\hline $\mathrm{C} 7$ & $4155(5)$ & 5119(9) & $6244(5)$ & $22.9(12)$ \\
\hline $\mathrm{C} 8$ & $3841(6)$ & $3735(9)$ & $6357(5)$ & $23.6(15)$ \\
\hline $\mathrm{C} 3$ & $1320(5)$ & 6511(8) & $5724(4)$ & $18(2)$ \\
\hline C13 & $5594(6)$ & $5208(9)$ & $6940(5)$ & $25(2)$ \\
\hline $\mathrm{C} 4$ & $2350(5)$ & 6612(9) & $5805(5)$ & $19(2)$ \\
\hline $\mathrm{C} 12$ & $4160(5)$ & 7082(9) & $5493(5)$ & $23(2)$ \\
\hline $\mathrm{C} 15$ & 6959(6) & 4979(9) & $6435(5)$ & $26(2)$ \\
\hline C5 & $2770(5)$ & 5643(9) & $5400(4)$ & $16.0(19)$ \\
\hline $\mathrm{C} 2$ & $942(5)$ & 5091(9) & $5759(5)$ & $20(2)$ \\
\hline C6 & $3810(5)$ & $5663(9)$ & $5568(4)$ & $19(2)$ \\
\hline $\mathrm{C} 16$ & 7881(6) & $4767(8)$ & $6515(5)$ & $25(2)$ \\
\hline $\mathrm{C} 10$ & $3260(6)$ & $4130(13)$ & 7393(4) & $30(2)$ \\
\hline
\end{tabular}




\begin{tabular}{|c|c|c|c|c|}
\hline \multirow[b]{2}{*}{ C9 } & \multicolumn{4}{|c|}{ D. Rhoades, * et al. } \\
\hline & $3473(5)$ & $3309(10)$ & $6827(4)$ & $23.6(15)$ \\
\hline $\mathrm{C} 1$ & $1371(5)$ & $4156(11)$ & $6295(4)$ & $24(2)$ \\
\hline $\mathrm{C} 14$ & $6565(6)$ & 4989(9) & $6983(5)$ & $24(2)$ \\
\hline $\mathrm{C} 11$ & $2253(6)$ & $4083(12)$ & $7374(4)$ & $30(2)$ \\
\hline C18 & $8010(6)$ & $4519(12)$ & $7648(6)$ & $47(3)$ \\
\hline $\mathrm{C} 17$ & $8377(6)$ & $4550(10)$ & $7104(5)$ & $34(3)$ \\
\hline $\mathrm{C} 20$ & $3783(7)$ & $3539(13)$ & $8012(6)$ & $47(3)$ \\
\hline C19 & $7080(6)$ & $4758(11)$ & $7576(6)$ & $42(3)$ \\
\hline $\mathrm{C} 22$ & $1970(6)$ & $4655(12)$ & $7968(5)$ & $42(3)$ \\
\hline $\mathrm{C} 21$ & $2132(11)$ & $6161(15)$ & $8077(7)$ & $71(4)$ \\
\hline $\mathrm{C} 23$ & $1583(6)$ & $3881(15)$ & $8325(6)$ & $54(4)$ \\
\hline $\mathrm{C} 29$ & $268(13)$ & $3080(20)$ & $9135(12)$ & $75(6)$ \\
\hline $\mathrm{C} 30$ & $410(14)$ & $6130(20)$ & $8943(14)$ & $86(7)$ \\
\hline C30A & $260(30)$ & $5030(40)$ & $8990(30)$ & $86(7)$ \\
\hline $\mathrm{Si}$ & $1160(8)$ & $3604(15)$ & $9063(6)$ & $55.0(15)$ \\
\hline C29A & $560(30)$ & $1870(30)$ & $9100(30)$ & $81(14)$ \\
\hline $\mathrm{C} 26$ & $2057(7)$ & $4615(18)$ & $9722(5)$ & $118(6)$ \\
\hline $\mathrm{C} 25$ & 1889(9) & $5328(18)$ & $10250(7)$ & $134(7)$ \\
\hline $\mathrm{C} 24$ & 2571(12) & $5520(20)$ & $10784(6)$ & 193(13) \\
\hline $\mathrm{C} 28$ & $3421(10)$ & $4990(20)$ & $10790(6)$ & $183(12)$ \\
\hline C31 & $3588(7)$ & $4280(20)$ & $10262(8)$ & $167(10)$ \\
\hline $\mathrm{C} 27$ & $2906(9)$ & $4090(20)$ & $9729(6)$ & $145(8)$ \\
\hline
\end{tabular}


D. Rhoades, * et al.

Table S20. Anisotropic Displacement Parameters $\left(\AA^{2} \times 10^{3}\right)$ for 24. The Anisotropic displacement factor exponent takes the form: $-2 \pi^{2}\left[h^{2} a^{* 2} U_{11}+2 h k a * b * U_{12}+\ldots\right]$.

\begin{tabular}{|c|c|c|c|c|c|c|}
\hline Atom & $\mathrm{U}_{11}$ & $\mathbf{U}_{22}$ & $\mathbf{U}_{33}$ & $\mathbf{U}_{23}$ & $\mathbf{U}_{13}$ & $\mathbf{U}_{12}$ \\
\hline $\mathrm{Br} 1$ & $18.4(4)$ & $81.9(8)$ & $57.7(9)$ & $6.1(8)$ & $-2.7(5)$ & $13.9(6)$ \\
\hline Si1' & $58(2)$ & $71(4)$ & $46(2)$ & $6(3)$ & $33.3(19)$ & $3(3)$ \\
\hline $\mathrm{O5}$ & $11(2)$ & $34(3)$ & $24(3)$ & $0(2)$ & $3(2)$ & $3(2)$ \\
\hline $\mathrm{O} 4$ & $13(3)$ & $33(3)$ & $21(4)$ & $-7(3)$ & $5(3)$ & $2(2)$ \\
\hline $\mathrm{O} 3$ & $16(3)$ & $31(3)$ & $23(4)$ & $1(3)$ & $1(3)$ & $1(3)$ \\
\hline $\mathrm{O} 2$ & $24(3)$ & $35(3)$ & $19(4)$ & $3(3)$ & $3(3)$ & $-1(3)$ \\
\hline O1 & $28(3)$ & $28(4)$ & $43(5)$ & $8(3)$ & $-5(3)$ & $-4(3)$ \\
\hline O6 & $23(3)$ & $72(5)$ & $19(4)$ & $-9(4)$ & $-3(3)$ & $7(3)$ \\
\hline $\mathrm{C} 7$ & $11(2)$ & $34(3)$ & $24(3)$ & $0(2)$ & $3(2)$ & $3(2)$ \\
\hline $\mathrm{C} 8$ & $17(3)$ & $36(4)$ & $14(4)$ & $-2(3)$ & $-4(3)$ & $1(3)$ \\
\hline C3 & $10(4)$ & $23(4)$ & $21(6)$ & $-7(4)$ & $9(4)$ & $2(3)$ \\
\hline C13 & $21(5)$ & $27(5)$ & $23(7)$ & $1(4)$ & $-4(5)$ & $0(4)$ \\
\hline $\mathrm{C} 4$ & $9(4)$ & $25(5)$ & $24(6)$ & $3(4)$ & $4(4)$ & $-2(3)$ \\
\hline $\mathrm{C} 12$ & $14(4)$ & $25(5)$ & $28(6)$ & $1(4)$ & $1(4)$ & $-4(4)$ \\
\hline C15 & $21(4)$ & $22(4)$ & $30(6)$ & $-3(4)$ & $-7(5)$ & $2(4)$ \\
\hline C5 & $10(4)$ & $24(4)$ & $14(5)$ & $-3(4)$ & $4(4)$ & $0(3)$ \\
\hline $\mathrm{C} 2$ & $11(4)$ & $27(5)$ & $21(6)$ & $-5(4)$ & $0(4)$ & $-9(3)$ \\
\hline C6 & $14(4)$ & $24(5)$ & $20(6)$ & $-5(4)$ & $6(4)$ & $-6(3)$ \\
\hline C16 & $19(4)$ & $23(5)$ & $31(7)$ & $-6(4)$ & $2(5)$ & $0(3)$ \\
\hline C10 & $32(4)$ & $50(6)$ & $9(5)$ & $10(6)$ & $4(4)$ & $0(5)$ \\
\hline
\end{tabular}




\begin{tabular}{|c|c|c|c|c|c|c|}
\hline \multirow[b]{2}{*}{ C9 } & \multicolumn{6}{|c|}{ D. Rhoades, * et al. } \\
\hline & $17(3)$ & $36(4)$ & $14(4)$ & $-2(3)$ & $-4(3)$ & $1(3)$ \\
\hline $\mathrm{C} 1$ & $15(3)$ & $32(5)$ & $28(6)$ & $7(5)$ & $8(4)$ & $1(4)$ \\
\hline C14 & $16(4)$ & $31(5)$ & $21(6)$ & $-2(4)$ & $-7(5)$ & $1(4)$ \\
\hline C11 & $28(4)$ & $43(6)$ & $17(6)$ & $10(5)$ & $1(4)$ & $-2(5)$ \\
\hline $\mathrm{C} 18$ & $29(5)$ & $55(8)$ & $49(8)$ & $16(6)$ & $-12(5)$ & $9(5)$ \\
\hline $\mathrm{C} 17$ & $20(4)$ & $40(7)$ & $40(7)$ & $-2(5)$ & $1(5)$ & $2(4)$ \\
\hline $\mathrm{C} 20$ & $27(5)$ & $80(8)$ & $32(8)$ & $7(6)$ & $-3(6)$ & $0(5)$ \\
\hline C19 & $23(5)$ & $63(8)$ & $40(8)$ & $16(6)$ & $4(5)$ & $1(4)$ \\
\hline $\mathrm{C} 22$ & $21(4)$ & $77(9)$ & $27(6)$ & $13(6)$ & $6(5)$ & $9(5)$ \\
\hline $\mathrm{C} 21$ & $94(11)$ & $77(10)$ & $40(9)$ & $-26(8)$ & $9(8)$ & $-1(9)$ \\
\hline $\mathrm{C} 23$ & $22(5)$ & $96(12)$ & $42(8)$ & $13(7)$ & $5(6)$ & $8(6)$ \\
\hline $\mathrm{C} 29$ & $63(9)$ & $84(9)$ & $93(18)$ & $11(9)$ & $49(11)$ & $3(7)$ \\
\hline $\mathrm{C} 30$ & $73(9)$ & $77(7)$ & $117(19)$ & $10(9)$ & $42(11)$ & $12(7)$ \\
\hline C30A & $73(9)$ & $77(7)$ & $117(19)$ & $10(9)$ & $42(11)$ & $12(7)$ \\
\hline $\mathrm{Si}$ & $58(2)$ & $71(4)$ & $46(2)$ & $6(3)$ & $33.3(19)$ & $3(3)$ \\
\hline C29A & $74(18)$ & $78(9)$ & $100(40)$ & $15(11)$ & $30(20)$ & $-2(9)$ \\
\hline $\mathrm{C} 26$ & $92(6)$ & $198(17)$ & $61(6)$ & $16(8)$ & $10(5)$ & $-9(8)$ \\
\hline $\mathrm{C} 25$ & 126(9) & $204(17)$ & $74(7)$ & $0(9)$ & $24(6)$ & $-20(10)$ \\
\hline $\mathrm{C} 24$ & $149(11)$ & $310(30)$ & $107(10)$ & $-37(15)$ & $-2(8)$ & $9(14)$ \\
\hline $\mathrm{C} 28$ & $143(11)$ & $280(30)$ & $118(12)$ & $-28(15)$ & $5(9)$ & $-3(14)$ \\
\hline $\mathrm{C} 31$ & $115(8)$ & $260(30)$ & $106(11)$ & $-17(15)$ & $-13(8)$ & $7(12)$ \\
\hline $\mathrm{C} 27$ & $97(6)$ & $240(20)$ & $91(9)$ & $-6(12)$ & $2(6)$ & $7(10)$ \\
\hline
\end{tabular}


D. Rhoades, * et al.

Table S21. Bond Lengths for 24.

\begin{tabular}{|c|c|c|c|c|c|}
\hline Atom & Atom & Length/Å & Atom & Atom & Length/Å \\
\hline $\mathrm{Br} 1$ & $\mathrm{C} 17$ & $1.900(9)$ & C15 & $\mathrm{C} 14$ & $1.415(14)$ \\
\hline Si1' & $\mathrm{C} 23$ & $1.932(13)$ & $\mathrm{C} 5$ & C6 & $1.553(10)$ \\
\hline Si1' & C29 & $1.943(15)$ & $\mathrm{C} 2$ & $\mathrm{C} 1$ & $1.507(13)$ \\
\hline Sil' & $\mathrm{C} 30$ & $1.934(16)$ & $\mathrm{C} 16$ & $\mathrm{C} 17$ & $1.346(14)$ \\
\hline Si1' & $\mathrm{C} 26$ & $1.781(11)$ & $\mathrm{C} 10$ & C9 & $1.537(14)$ \\
\hline O5 & $\mathrm{C} 7$ & $1.472(9)$ & $\mathrm{C} 10$ & $\mathrm{C} 11$ & $1.527(11)$ \\
\hline $\mathrm{O} 5$ & $\mathrm{C} 13$ & $1.347(11)$ & $\mathrm{C} 10$ & $\mathrm{C} 20$ & $1.513(14)$ \\
\hline $\mathrm{O} 4$ & C6 & $1.454(10)$ & C14 & C19 & $1.366(15)$ \\
\hline $\mathrm{O} 3$ & $\mathrm{C} 3$ & $1.435(11)$ & $\mathrm{C} 11$ & $\mathrm{C} 22$ & $1.522(14)$ \\
\hline $\mathrm{O} 2$ & $\mathrm{C} 1$ & $1.368(11)$ & $\mathrm{C} 18$ & $\mathrm{C} 17$ & $1.385(15)$ \\
\hline $\mathrm{O} 2$ & C11 & $1.458(11)$ & $\mathrm{C} 18$ & C19 & $1.414(13)$ \\
\hline $\mathrm{O} 1$ & $\mathrm{C} 1$ & $1.187(13)$ & $\mathrm{C} 22$ & $\mathrm{C} 21$ & $1.509(18)$ \\
\hline O6 & $\mathrm{C} 13$ & $1.199(12)$ & $\mathrm{C} 22$ & $\mathrm{C} 23$ & $1.297(15)$ \\
\hline C7 & $\mathrm{C} 8$ & $1.475(12)$ & $\mathrm{C} 23$ & $\mathrm{Si}$ & $1.835(17)$ \\
\hline C7 & C6 & $1.526(13)$ & C30A & $\mathrm{Si}$ & $1.943(17)$ \\
\hline $\mathrm{C} 8$ & C9 & $1.310(13)$ & $\mathrm{Si}$ & C29A & $1.938(18)$ \\
\hline C3 & $\mathrm{C} 4$ & $1.547(10)$ & $\mathrm{C} 26$ & $\mathrm{C} 25$ & 1.3900 \\
\hline $\mathrm{C} 3$ & $\mathrm{C} 2$ & $1.515(12)$ & $\mathrm{C} 26$ & $\mathrm{C} 27$ & 1.3900 \\
\hline $\mathrm{C} 13$ & C14 & $1.480(12)$ & $\mathrm{C} 25$ & C24 & 1.3900 \\
\hline $\mathrm{C} 4$ & $\mathrm{C} 5$ & $1.509(12)$ & $\mathrm{C} 24$ & $\mathrm{C} 28$ & 1.3900 \\
\hline $\mathrm{C} 12$ & C6 & $1.511(12)$ & $\mathrm{C} 28$ & $\mathrm{C} 31$ & 1.3900 \\
\hline
\end{tabular}


D. Rhoades, ${ }^{*}$ et al.

$\begin{array}{llllll}\mathrm{C} 15 & \mathrm{C} 16 & 1.395(12) & \mathrm{C} 31 & \mathrm{C} 27 & 1.3900\end{array}$

Table S22. Bond Angles for 24.

\begin{tabular}{|c|c|c|c|c|c|c|c|}
\hline Atom & Atom & Atom & Angle ${ }^{\circ}$ & Atom & Atom & Atom & Angle ${ }^{\circ}$ \\
\hline $\mathrm{C} 29$ & Sil' & $\mathrm{C} 23$ & 101.4(9) & $\mathrm{C} 8$ & C9 & C10 & $128.5(9)$ \\
\hline $\mathrm{C} 30$ & Si1' & $\mathrm{C} 23$ & $116.0(10)$ & $\mathrm{O} 2$ & $\mathrm{C} 1$ & $\mathrm{C} 2$ & 112.1(9) \\
\hline $\mathrm{C} 30$ & Si1' & $\mathrm{C} 29$ & $102.9(10)$ & $\mathrm{O} 1$ & $\mathrm{C} 1$ & $\mathrm{O} 2$ & $124.2(8)$ \\
\hline $\mathrm{C} 26$ & Si1' & $\mathrm{C} 23$ & 108.1(6) & $\mathrm{O} 1$ & $\mathrm{C} 1$ & $\mathrm{C} 2$ & 123.7(9) \\
\hline $\mathrm{C} 26$ & Sil' & $\mathrm{C} 29$ & $115.1(10)$ & $\mathrm{C} 15$ & $\mathrm{C} 14$ & $\mathrm{C} 13$ & 122.3(9) \\
\hline $\mathrm{C} 26$ & Si1' & $\mathrm{C} 30$ & $113.0(11)$ & C19 & $\mathrm{C} 14$ & $\mathrm{C} 13$ & $117.5(9)$ \\
\hline $\mathrm{C} 13$ & O5 & $\mathrm{C} 7$ & $115.7(7)$ & C19 & C14 & $\mathrm{C} 15$ & 120.1(8) \\
\hline $\mathrm{C} 1$ & $\mathrm{O} 2$ & C11 & $117.8(8)$ & $\mathrm{O} 2$ & $\mathrm{C} 11$ & $\mathrm{C} 10$ & 108.9(7) \\
\hline O5 & $\mathrm{C} 7$ & $\mathrm{C} 8$ & 108.4(7) & $\mathrm{O} 2$ & $\mathrm{C} 11$ & $\mathrm{C} 22$ & 107.6(8) \\
\hline O5 & C7 & C6 & $106.0(7)$ & $\mathrm{C} 10$ & C11 & $\mathrm{C} 22$ & 114.7(8) \\
\hline $\mathrm{C} 8$ & C7 & C6 & $114.5(8)$ & $\mathrm{C} 17$ & $\mathrm{C} 18$ & C19 & 118.1(10) \\
\hline C9 & $\mathrm{C} 8$ & C7 & 128.4(9) & C16 & $\mathrm{C} 17$ & $\mathrm{Br} 1$ & $120.1(8)$ \\
\hline $\mathrm{O} 3$ & $\mathrm{C} 3$ & $\mathrm{C} 4$ & $108.2(7)$ & C16 & $\mathrm{C} 17$ & $\mathrm{C} 18$ & $122.5(9)$ \\
\hline $\mathrm{O} 3$ & $\mathrm{C} 3$ & $\mathrm{C} 2$ & 106.3(7) & $\mathrm{C} 18$ & $\mathrm{C} 17$ & $\mathrm{Br} 1$ & 117.4(8) \\
\hline $\mathrm{C} 2$ & $\mathrm{C} 3$ & $\mathrm{C} 4$ & 116.1(7) & $\mathrm{C} 14$ & C19 & $\mathrm{C} 18$ & $120.3(10)$ \\
\hline $\mathrm{O} 5$ & $\mathrm{C} 13$ & $\mathrm{C} 14$ & 111.2(9) & $\mathrm{C} 21$ & $\mathrm{C} 22$ & C11 & $115.1(10)$ \\
\hline O6 & $\mathrm{C} 13$ & O5 & $124.7(8)$ & $\mathrm{C} 23$ & $\mathrm{C} 22$ & C11 & $120.6(11)$ \\
\hline O6 & $\mathrm{C} 13$ & C14 & $124.0(9)$ & $\mathrm{C} 23$ & $\mathrm{C} 22$ & $\mathrm{C} 21$ & $124.2(12)$ \\
\hline
\end{tabular}




\begin{tabular}{|c|c|c|c|c|c|c|c|}
\hline \multirow[b]{2}{*}{$\mathrm{C} 5$} & \multicolumn{7}{|c|}{ D. Rhoades, * et al. } \\
\hline & $\mathrm{C} 4$ & $\mathrm{C} 3$ & $115.7(7)$ & $\mathrm{C} 22$ & $\mathrm{C} 23$ & Si1' & $124.7(11)$ \\
\hline $\mathrm{C} 16$ & $\mathrm{C} 15$ & $\mathrm{C} 14$ & $118.9(9)$ & $\mathrm{C} 22$ & $\mathrm{C} 23$ & $\mathrm{Si}$ & $150.3(13)$ \\
\hline $\mathrm{C} 4$ & $\mathrm{C} 5$ & C6 & $113.0(7)$ & $\mathrm{C} 23$ & $\mathrm{Si}$ & C30A & $100.9(18)$ \\
\hline $\mathrm{C} 1$ & $\mathrm{C} 2$ & $\mathrm{C} 3$ & 119.1(7) & $\mathrm{C} 23$ & $\mathrm{Si}$ & C29A & $114.3(18)$ \\
\hline $\mathrm{O} 4$ & C6 & $\mathrm{C} 7$ & $110.4(7)$ & C29A & $\mathrm{Si}$ & C30A & $108(2)$ \\
\hline $\mathrm{O} 4$ & C6 & $\mathrm{C} 12$ & $106.8(7)$ & $\mathrm{C} 25$ & $\mathrm{C} 26$ & Sil' & 115.3(9) \\
\hline $\mathrm{O} 4$ & C6 & $\mathrm{C} 5$ & $106.9(6)$ & $\mathrm{C} 25$ & $\mathrm{C} 26$ & $\mathrm{C} 27$ & 120.0 \\
\hline $\mathrm{C} 7$ & C6 & $\mathrm{C} 5$ & $110.8(7)$ & $\mathrm{C} 27$ & $\mathrm{C} 26$ & Si1' & $124.7(9)$ \\
\hline $\mathrm{C} 12$ & C6 & $\mathrm{C} 7$ & 111.1(7) & $\mathrm{C} 26$ & $\mathrm{C} 25$ & $\mathrm{C} 24$ & 120.0 \\
\hline $\mathrm{C} 12$ & C6 & C5 & $110.7(7)$ & $\mathrm{C} 28$ & $\mathrm{C} 24$ & $\mathrm{C} 25$ & 120.0 \\
\hline $\mathrm{C} 17$ & $\mathrm{C} 16$ & $\mathrm{C} 15$ & $120.1(10)$ & $\mathrm{C} 24$ & $\mathrm{C} 28$ & $\mathrm{C} 31$ & 120.0 \\
\hline $\mathrm{C} 11$ & $\mathrm{C} 10$ & C9 & $109.4(8)$ & $\mathrm{C} 28$ & C31 & $\mathrm{C} 27$ & 120.0 \\
\hline $\mathrm{C} 20$ & $\mathrm{C} 10$ & $\mathrm{C} 9$ & 109.0(9) & C31 & $\mathrm{C} 27$ & $\mathrm{C} 26$ & 120.0 \\
\hline C20 & $\mathrm{C} 10$ & C11 & $111.2(8)$ & & & & \\
\hline
\end{tabular}

Table S23. Torsion Angles for 24.

\begin{tabular}{|c|c|c|c|c|c|c|c|c|c|}
\hline $\mathbf{A}$ & B & C & D & Angle ${ }^{\circ}$ & $\mathbf{A}$ & B & $\mathrm{C}$ & D & Angle $^{\circ}$ \\
\hline Sil' & $\mathrm{C} 26$ & $\mathrm{C} 25$ & $\mathrm{C} 24$ & $\begin{array}{l}- \\
178.0(12)\end{array}$ & $\mathrm{C} 16$ & $\mathrm{C} 15$ & C14 & C19 & $-1.7(14)$ \\
\hline Sil' & $\mathrm{C} 26$ & $\mathrm{C} 27$ & $\mathrm{C} 31$ & $177.8(14)$ & $\mathrm{C} 10$ & $\mathrm{C} 11$ & $\mathrm{C} 22$ & $\mathrm{C} 21$ & $65.2(13)$ \\
\hline O5 & $\mathrm{C} 7$ & $\mathrm{C} 8$ & C9 & $112.3(10)$ & $\mathrm{C} 10$ & $\mathrm{C} 11$ & $\mathrm{C} 22$ & $\mathrm{C} 23$ & $\begin{array}{l}- \\
117.6(11)\end{array}$ \\
\hline O5 & $\mathrm{C} 7$ & C6 & $\mathrm{O} 4$ & $57.5(8)$ & C9 & $\mathrm{C} 10$ & $\mathrm{C} 11$ & $\mathrm{O} 2$ & $-69.0(11)$ \\
\hline
\end{tabular}


D. Rhoades, ${ }^{*}$ et al.

$\begin{array}{llllllllll}\mathrm{O} 5 & \mathrm{C} 7 & \mathrm{C} 6 & \mathrm{C} 12 & -60.8(9) & \mathrm{C} 9 & \mathrm{C} 10 & \mathrm{C} 11 & \mathrm{C} 22 & 170.3(9)\end{array}$

$\begin{array}{llllllllll}\mathrm{O} 5 & \mathrm{C} 7 & \mathrm{C} 6 & \mathrm{C} 5 & 175.8(7) & \mathrm{C} 1 & \mathrm{O} 2 & \mathrm{C} 11 & \mathrm{C} 10 & 102.9(9)\end{array}$

$\begin{array}{llllllllll}\mathrm{O} 5 & \mathrm{C} 13 & \mathrm{C} 14 & \mathrm{C} 15 & 13.7(13) & \mathrm{C} 1 & \mathrm{O} 2 & \mathrm{C} 11 & \mathrm{C} 22 & -132.1(8)\end{array}$

$\begin{array}{llllllllll}\text { O5 } & \mathrm{C} 13 & \mathrm{C} 14 & \mathrm{C} 19 & -164.8(8) & \mathrm{C} 14 & \mathrm{C} 15 & \mathrm{C} 16 & \mathrm{C} 17 & 1.2(13)\end{array}$

$\begin{array}{llllllllll}\mathrm{O} 3 & \mathrm{C} 3 & \mathrm{C} 4 & \mathrm{C} 5 & -70.6(9) & \mathrm{C} 11 & \mathrm{O} 2 & \mathrm{C} 1 & \mathrm{O} 1 & 3.7(11)\end{array}$

$\begin{array}{llllllllll}\mathrm{O} 3 & \mathrm{C} 3 & \mathrm{C} 2 & \mathrm{C} 1 & 167.4(7) & \mathrm{C} 11 & \mathrm{O} 2 & \mathrm{C} 1 & \mathrm{C} 2 & -178.6(6)\end{array}$

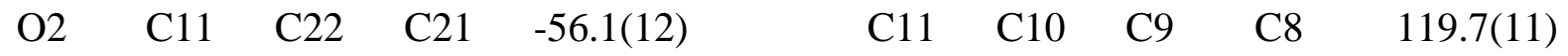

$\begin{array}{llllllllll}\mathrm{O} 2 & \mathrm{C} 11 & \mathrm{C} 22 & \mathrm{C} 23 & 121.0(10) & \mathrm{C} 11 & \mathrm{C} 22 & \mathrm{C} 23 & \mathrm{Si} 1^{\prime} & -176.6(7)\end{array}$

$\begin{array}{llllllllll}\text { O6 } & \mathrm{C} 13 & \mathrm{C} 14 & \mathrm{C} 15 & -168.5(9) & \mathrm{C} 11 & \mathrm{C} 22 & \mathrm{C} 23 & \mathrm{Si} & 170.7(15)\end{array}$

$\begin{array}{llllllllll}\text { O6 } & \mathrm{C} 13 & \mathrm{C} 14 & \mathrm{C} 19 & 13.1(15) & \mathrm{C} 17 & \mathrm{C} 18 & \mathrm{C} 19 & \mathrm{C} 14 & 1.1(17)\end{array}$

$\begin{array}{llllllllll}\mathrm{C} 7 & \mathrm{O} 5 & \mathrm{C} 13 & \mathrm{O} 6 & -5.0(13) & \mathrm{C} 20 & \mathrm{C} 10 & \mathrm{C} 9 & \mathrm{C} 8 & \\ & & & & & & & & & \end{array}$

$\begin{array}{llllllllll}\mathrm{C} 7 & \mathrm{O} 5 & \mathrm{C} 13 & \mathrm{C} 14 & 172.8(7) & \mathrm{C} 20 & \mathrm{C} 10 & \mathrm{C} 11 & \mathrm{O} 2 & 170.6(9)\end{array}$

$\begin{array}{llllllllll}\mathrm{C} 7 & \mathrm{C} 8 & \mathrm{C} 9 & \mathrm{C} 10 & 0.2(15) & \mathrm{C} 20 & \mathrm{C} 10 & \mathrm{C} 11 & \mathrm{C} 22 & 49.9(14)\end{array}$

$\begin{array}{llllllllll}\mathrm{C} 8 & \mathrm{C} 7 & \mathrm{C} 6 & \mathrm{O} 4 & -61.9(8) & \mathrm{C} 19 & \mathrm{C} 18 & \mathrm{C} 17 & \mathrm{Br} 1 & 178.8(8)\end{array}$

$\begin{array}{llllllllll}\mathrm{C} 8 & \mathrm{C} 7 & \mathrm{C} 6 & \mathrm{C} 12 & 179.8(7) & \mathrm{C} 19 & \mathrm{C} 18 & \mathrm{C} 17 & \mathrm{C} 16 & -1.6(17)\end{array}$

$\begin{array}{llllllllll}\mathrm{C} 8 & \mathrm{C} 7 & \mathrm{C} 6 & \mathrm{C} 5 & 56.4(9) & \mathrm{C} 22 & \mathrm{C} 23 & \mathrm{Si} & \mathrm{C} 30 \mathrm{~A} & 61(3)\end{array}$

$\begin{array}{llllllllll}\mathrm{C} 3 & \mathrm{C} 4 & \mathrm{C} 5 & \mathrm{C} 6 & -172.5(7) & \mathrm{C} 22 & \mathrm{C} 23 & \mathrm{Si} & \mathrm{C} 29 \mathrm{~A} & 176(2)\end{array}$

$\begin{array}{llllllllll}\mathrm{C} 3 & \mathrm{C} 2 & \mathrm{C} 1 & \mathrm{O} 2 & 26.1(10) & \mathrm{C} 21 & \mathrm{C} 22 & \mathrm{C} 23 & \mathrm{Si} 1 & 0.3(16)\end{array}$

$\begin{array}{llllllllll}\mathrm{C} 3 & \mathrm{C} 2 & \mathrm{C} 1 & \mathrm{O} 1 & -156.3(8) & \mathrm{C} 21 & \mathrm{C} 22 & \mathrm{C} 23 & \mathrm{Si} & -12(2)\end{array}$

$\begin{array}{llllllllll}\mathrm{C} 13 & \mathrm{O} 5 & \mathrm{C} 7 & \mathrm{C} 8 & -83.8(10) & \mathrm{C} 23 & \mathrm{Si} 1 & \mathrm{C} 26 & \mathrm{C} 25 & 166.2(9)\end{array}$

$\begin{array}{llllllllll}\mathrm{C} 13 & \mathrm{O} 5 & \mathrm{C} 7 & \mathrm{C} 6 & 152.8(7) & \text { C23 } & \text { Si1 } & \mathrm{C} 26 & \mathrm{C} 27 & -11.6(12)\end{array}$

$\begin{array}{llllllllll}\text { C13 } & \text { C14 } & \text { C19 } & \text { C18 } & 179.0(10) & \text { C29 } & \text { Si1 } & \text { C26 } & \text { C25 } & -81.3(12)\end{array}$ 

D. Rhoades, * et al.

\begin{tabular}{|c|c|c|c|c|c|c|c|c|c|}
\hline $\mathrm{C} 4$ & C3 & $\mathrm{C} 2$ & $\mathrm{C} 1$ & $47.0(11)$ & C29 & Si1' & C26 & C27 & $100.9(13)$ \\
\hline $\mathrm{C} 4$ & C5 & C6 & $\mathrm{O} 4$ & $-173.7(7)$ & C30 & Si1' & $\mathrm{C} 26$ & $\mathrm{C} 25$ & $36.4(13)$ \\
\hline $\mathrm{C} 4$ & $\mathrm{C} 5$ & C6 & $\mathrm{C} 7$ & $65.9(9)$ & C30 & Sil' & $\mathrm{C} 26$ & C27 & $\begin{array}{l}- \\
141.4(12)\end{array}$ \\
\hline $\mathrm{C} 4$ & $\mathrm{C} 5$ & C6 & $\mathrm{C} 12$ & $-57.8(10)$ & $\mathrm{C} 26$ & $\mathrm{C} 25$ & $\mathrm{C} 24$ & C28 & 0.0 \\
\hline C15 & C16 & $\mathrm{C} 17$ & $\mathrm{Br} 1$ & $180.0(7)$ & $\mathrm{C} 25$ & $\mathrm{C} 26$ & $\mathrm{C} 27$ & C31 & 0.0 \\
\hline C15 & C16 & $\mathrm{C} 17$ & C18 & $0.4(15)$ & $\mathrm{C} 25$ & $\mathrm{C} 24$ & $\mathrm{C} 28$ & C31 & 0.0 \\
\hline C15 & $\mathrm{C} 14$ & C19 & C18 & $0.5(16)$ & $\mathrm{C} 24$ & C28 & C31 & C27 & 0.0 \\
\hline $\mathrm{C} 2$ & $\mathrm{C} 3$ & $\mathrm{C} 4$ & $\mathrm{C} 5$ & $48.8(11)$ & $\mathrm{C} 28$ & C31 & $\mathrm{C} 27$ & C26 & 0.0 \\
\hline C6 & C7 & $\mathrm{C} 8$ & $\mathrm{C} 9$ & $\begin{array}{l}- \\
129.6(10)\end{array}$ & $\mathrm{C} 27$ & $\mathrm{C} 26$ & $\mathrm{C} 25$ & $\mathrm{C} 24$ & 0.0 \\
\hline C16 & C15 & C14 & C13 & $180.0(8)$ & & & & & \\
\hline
\end{tabular}

Table S24. Hydrogen Atom Coordinates $\left(\AA ̊ \times 10^{4}\right)$ and Isotropic Displacement Parameters $\left(\AA^{2} \times 10^{3}\right)$ for 24.

\begin{tabular}{lcccc}
\hline & Atom & \multicolumn{1}{c}{$\boldsymbol{y}$} & & $\boldsymbol{z}(\mathbf{e q})$ \\
\hline H4 & 4682 & 4825 & 5165 & 33 \\
H3 & 367 & 6882 & 5029 & 36 \\
H7 & 3981 & 5761 & 6562 & 28 \\
H8 & 3916 & 3066 & 6050 & 28 \\
H3A & 1127 & 7091 & 6058 & 21 \\
H4A & 2503 & 7555 & 5701 & 23 \\
H4B & 2622 & 6447 & 6261 & 23
\end{tabular}




\begin{tabular}{|c|c|c|c|c|}
\hline \multirow[b]{2}{*}{$\mathrm{H} 12 \mathrm{~A}$} & \multicolumn{4}{|c|}{ D. Rhoades, * et al. } \\
\hline & 4817 & 7066 & 5582 & 34 \\
\hline $\mathrm{H} 12 \mathrm{~B}$ & 3954 & 7699 & 5795 & 34 \\
\hline $\mathrm{H} 12 \mathrm{C}$ & 3937 & 7402 & 5054 & 34 \\
\hline H15 & 6603 & 5115 & 6020 & 31 \\
\hline H5A & 2568 & 5886 & 4943 & 19 \\
\hline H5B & 2559 & 4706 & 5460 & 19 \\
\hline $\mathrm{H} 2 \mathrm{~A}$ & 303 & 5188 & 5782 & 25 \\
\hline $\mathrm{H} 2 \mathrm{~B}$ & 962 & 4625 & 5350 & 25 \\
\hline H16 & 8158 & 4775 & 6153 & 30 \\
\hline H10 & 3446 & 5099 & 7355 & 37 \\
\hline H9 & 3317 & 2371 & 6817 & 28 \\
\hline H11 & 2050 & 3113 & 7319 & 36 \\
\hline H18 & 8372 & 4342 & 8058 & 57 \\
\hline $\mathrm{H} 20 \mathrm{~A}$ & 4425 & 3592 & 8013 & 71 \\
\hline $\mathrm{H} 20 \mathrm{~B}$ & 3611 & 2584 & 8048 & 71 \\
\hline $\mathrm{H} 20 \mathrm{C}$ & 3651 & 4058 & 8375 & 71 \\
\hline H19 & 6811 & 4759 & 7942 & 51 \\
\hline $\mathrm{H} 21 \mathrm{~A}$ & 2775 & 6326 & 8224 & 107 \\
\hline $\mathrm{H} 21 \mathrm{~B}$ & 1809 & 6482 & 8403 & 107 \\
\hline $\mathrm{H} 21 \mathrm{C}$ & 1919 & 6655 & 7675 & 107 \\
\hline $\mathrm{H} 23$ & 1516 & 2943 & 8216 & 64 \\
\hline $\mathrm{H} 23 \mathrm{~A}$ & 1458 & 3057 & 8090 & 64 \\
\hline $\mathrm{H} 29 \mathrm{~A}$ & -213 & 3096 & 8753 & 113 \\
\hline
\end{tabular}




\begin{tabular}{|c|c|c|c|c|}
\hline \multicolumn{5}{|c|}{ D. Rhoades, ${ }^{*}$ et al. } \\
\hline H29B & 565 & 2194 & 9172 & 113 \\
\hline $\mathrm{H} 29 \mathrm{C}$ & 15 & 3252 & 9516 & 113 \\
\hline H30A & -86 & 6012 & 8575 & 129 \\
\hline H30B & 168 & 6304 & 9329 & 129 \\
\hline $\mathrm{H} 30 \mathrm{C}$ & 779 & 6907 & 8865 & 129 \\
\hline H30D & -22 & 5000 & 9365 & 129 \\
\hline H30E & 550 & 5923 & 8975 & 129 \\
\hline $\mathrm{H} 30 \mathrm{~F}$ & -191 & 4898 & 8601 & 129 \\
\hline H29D & 355 & 1800 & 9504 & 121 \\
\hline H29E & 45 & 1812 & 8740 & 121 \\
\hline $\mathrm{H} 29 \mathrm{~F}$ & 979 & 1127 & 9066 & 121 \\
\hline $\mathrm{H} 25$ & 1309 & 5686 & 10246 & 161 \\
\hline $\mathrm{H} 24$ & 2457 & 6004 & 11144 & 231 \\
\hline $\mathrm{H} 28$ & 3887 & 5123 & 11155 & 220 \\
\hline H31 & 4169 & 3923 & 10267 & 200 \\
\hline $\mathrm{H} 27$ & 3021 & 3604 & 9368 & 174 \\
\hline
\end{tabular}




\section{Rhoades, * et al.}

Table S25. Atomic Occupancy for $\mathbf{2 4}$.

\begin{tabular}{llllll}
\hline Atom & Occupancy & Atom & Occupancy & Atom & Occupancy \\
\hline Si1' & $0.679(8)$ & H23 & $0.679(8)$ & H23A & $0.321(8)$ \\
C29 & $0.679(8)$ & H29A & $0.679(8)$ & H29B & $0.679(8)$ \\
H29C & $0.679(8)$ & C30 & $0.679(8)$ & H30A & $0.679(8)$ \\
H30B & $0.679(8)$ & H30C & $0.679(8)$ & C30A & $0.321(8)$ \\
H30D & $0.321(8)$ & H30E & $0.321(8)$ & H30F & $0.321(8)$ \\
Si & $0.321(8)$ & C29A & $0.321(8)$ & H29D & $0.321(8)$ \\
H29E & $0.321(8)$ & H29F & $0.321(8)$ & & \\
\hline
\end{tabular}




\section{Rhoades, * et al.}

\section{References}

1. Still, W. C.; Kahn, M.; Mitra, A. Rapid Chromatographic Technique for Preparative Separations with Moderate Resolution. J. Org. Chem. 1978, 43, 2923-2925.

2. Fulmer, G. R.; Miller, A. J. M.; Sherden, N. H.; Gottlieb, H. E.; Nudelman, A.; Stoltz, B. M.; Bercaw, J. E.; Goldberg, K. I. NMR Chemical Shifts of Trace Impurities: Common Laboratory Solvents, Organics, and Gases in Deuterated Solvents Relevant to the Organometallic Chemist. Organometallics 2010, 29, 2176-2179.

3. $\quad$ Kanada, R. M.; Itoh, D.; Nagai, M.; Niijima, J.; Asai, N.; Mizui, Y.; Abe, S.; Kotake, Y. Total Synthesis of the Potent Antitumor Macrolides Pladienolide B and D. Angew. Chem., Int. Ed. 2007, 46, 4350-4355.

4. Ghosh, A. K.; Anderson, D. D. Enantioselective Total Synthesis of Pladienolide B: a Potent Spliceosome Inhibitor. Org. Lett. 2012, 14, 4730-4733.

5. Kumar, V. P.; Chandrasekhar, S. Enantioselective Synthesis of Pladienolide B and Truncated Analogues as New Anticancer Agents. Org. Lett. 2013, 15, 3610-3613.

6. (a) Müller, S.; Mayer, T.; Sasse, F.; Maier, M. E. Synthesis of a Pladienolide B Analogue with the Fully Functionalized Core Structure. Org. Lett. 2011, 13, 3940-3943; (b) Müller, S.; Sasse, F.; Maier, M. E. Synthesis of Pladienolide B and Its 7-Epimer with Insights into the Role of the Allylic Acetate. Eur. J. Org. Chem. 2014, 2014, 1025-1036.

7. Keaney, G. F.; Wang, J.; Gerard, B.; Arai, K.; Liu, X.; Zheng, G. Z.; Kira, K.; Tivitmahaisoon, P.; Prajapati, S.; Gearhart, N. C.; Kotake, Y.; Nagao, S.; Sonobe, R. M. K.; Miyano, M.; Murai, N. Pladienolide Pyridine Compounds and Methods of Use. US/2015/0329528A1, 2015.

8. (a) Doyle, M. P.; Shanklin, M. S. Highly Efficient Regioselective Silylcarbonylation of Alkynes Catalyzed by Dirhodium(II) Perfluorobutyrate. Organometallics 1993, 12, 11-12; (b) Jain, N. F.; Cirillo, P. F.; Schaus, J. V.; Panek, J. S. An Efficient Procedure for the Preparation of Chiral $\beta$ Substituted (E)-Crotylsilanes: Application of a Rhodium(II) Catalyzed Silylformylation of Terminal Alkynes. Tetrahedron Lett. 1995, 36, 8723-8726.

9. Matsuda, I.; Fukuta, Y.; Tsuchihashi, T.; Nagashima, H.; Itoh, K. Rhodium-Catalyzed Silylformylation of Acetylenic Bonds: Its Scope and Mechanistic Considerations. Organometallics 1997, 16, 4327-4345.

10. (a) Duboudin, J. G.; Jousseaume, B. Reactifs de Grignard vinyliques $\gamma$ fonctionnels: I. Reactivite des organomagnesiens vis-à-vis d'alcools $\alpha$ acetyleniques en presence d'halogenures cuivreux. $J$. Organomet. Chem. 1979, 168, 1-11; (b) Duboudin, J. G.; Jousseaume, B.; Bonakdar, A.; Saux, A. Reactifs de Grignard vinyliques $\gamma$ fonctionnels: II. Iodolyse, alkylation et arylation des iodo-alcools. $J$. Organomet. Chem. 1979, 168, 227-232; (c) Hiroya, K.; Takuma, K.; Inamoto, K.; Sakamoto, T. Synthesis of 6z-Pandanamine by Regioselective Cyclization Reaction of 2-En-4-Ynoic Acid Derivatives Promoted by Weak Base. Heterocycles 2009, 77, 493-505.

11. Kleinbeck, F.; Fettes, G. J.; Fader, L. D.; Carreira, E. M. Total Synthesis of Bafilomycin A1. Chem. Eur. J. 2012, 18, 3598-3610.

12. Larock, R. C.; Han, X. Palladium-Catalyzed Cross-Coupling of 2,5-Cyclohexadienyl-Substituted Aryl or Vinylic Iodides and Carbon or Heteroatom Nucleophiles. J. Org. Chem. 1999, 64, 1875-1887. 13. Brown, S. P.; Bedke, D. K.; Degraffenreid, M. R.; Fu, J.; Li, Z.; Gonzalez Lopez de Turiso, F.; Gonzalez Buenrostro, A.; Gribble, J., M. W.; Johnson, M. G.; Kohn, T. J.; Li, K.; Li, Y.; Lizarzaburu, M. E.; Rew, Y.; Taygerly, J.; Wang, Y.; Yan, X.; Yu, M.; Zhu, J.; Zancanella, M.; Jiao, X. Y.; Zhu, L.; Wang, X.; Medina, J. C.; Duquette, J. A.; Houze, J. B.; Vimolratana, M.; Cardozo, M.; Cheng, A. C. Compounds That Inhibit MCL-1 Protein. WO/2017/147410A1, 2017.

14. Haydl, A. M.; Breit, B. The Total Synthesis of Epothilone D as a Yardstick for Probing New Methodologies. Chem. Eur. 2017, 23, 541-545. 


\section{Rhoades, * et al.}

15. Ghosh, A.; Luo, J.; Liu, C.; Weltrowska, G.; Lemieux, C.; Chung, N. N.; Lu, Y. X.; Schiller, P. W. Novel Opioid Peptide Derived Antagonists Containing (2S)-2-Methyl-3-(2,6-dimethyl-4carbamoylphenyl)propanoic Acid [(2S)-Mdcp]. J. Med. Chem. 2008, 51, 5866-5870.

16. Featherston, A. L.; Miller, S. J. Synthesis and Evaluation of Phenylalanine-Derived Trifluoromethyl Ketones for Peptide-Based Oxidation Catalysis. Biorg. Med. Chem. 2016, 24, 48714874.

17. Clark, J. S.; Romiti, F. Total Syntheses of Amphidinolides T1, T3, and T4. Angew. Chem., Int. Ed. 2013, 52, 10072-10075.

18. Harrison, T. J.; Ho, S.; Leighton, J. L. Toward More "Ideal" Polyketide Natural Product Synthesis: A Step-Economical Synthesis of Zincophorin Methyl Ester. J. Am. Chem. Soc. 2011, 133, $7308-7311$.

19. (a) Hackman, B. M.; Lombardi, P. J.; Leighton, J. L. Highly Diastereo- and Enantioselective Reagents for Aldehyde Crotylation. Org. Lett. 2004, 6, 4375-4377; (b) Jecs, E.; Diver, S. T. Two EneYne Metathesis Approaches to the Total Synthesis of Amphidinolide P. Org. Lett. 2015, 17, 3510-3513. 20. Gao, X.; Townsend, I. A.; Krische, M. J. Enhanced anti-Diastereo- and Enantioselectivity in Alcohol-Mediated Carbonyl Crotylation Using an Isolable Single Component Iridium Catalyst. J. Org. Chem. 2011, 76, 2350-2354.

21. Hoye, T. R.; Jeffrey, C. S.; Shao, F. Mosher Ester Analysis for the Determination of Absolute Configuration of Stereogenic (Chiral) Carbinol Carbons. Nat. Protoc. 2007, 2, 2451-2458.

22. Hall, L. W.; Odom, J. D.; Ellis, P. D. Nuclear Magnetic-Resonance Investigation of Nature of Boron-Carbon Bond in Some Vinylboranes. J. Am. Chem. Soc. 1975, 97, 4527-4531.

23. Lee, D.; Kim, D.; Yun, J. Highly Enantioselective Conjugate Reduction of $\beta, \beta$-Disubstituted $\alpha, \beta$ Unsaturated Nitriles. Angew. Chem., Int. Ed. 2006, 45, 2785-2787. 